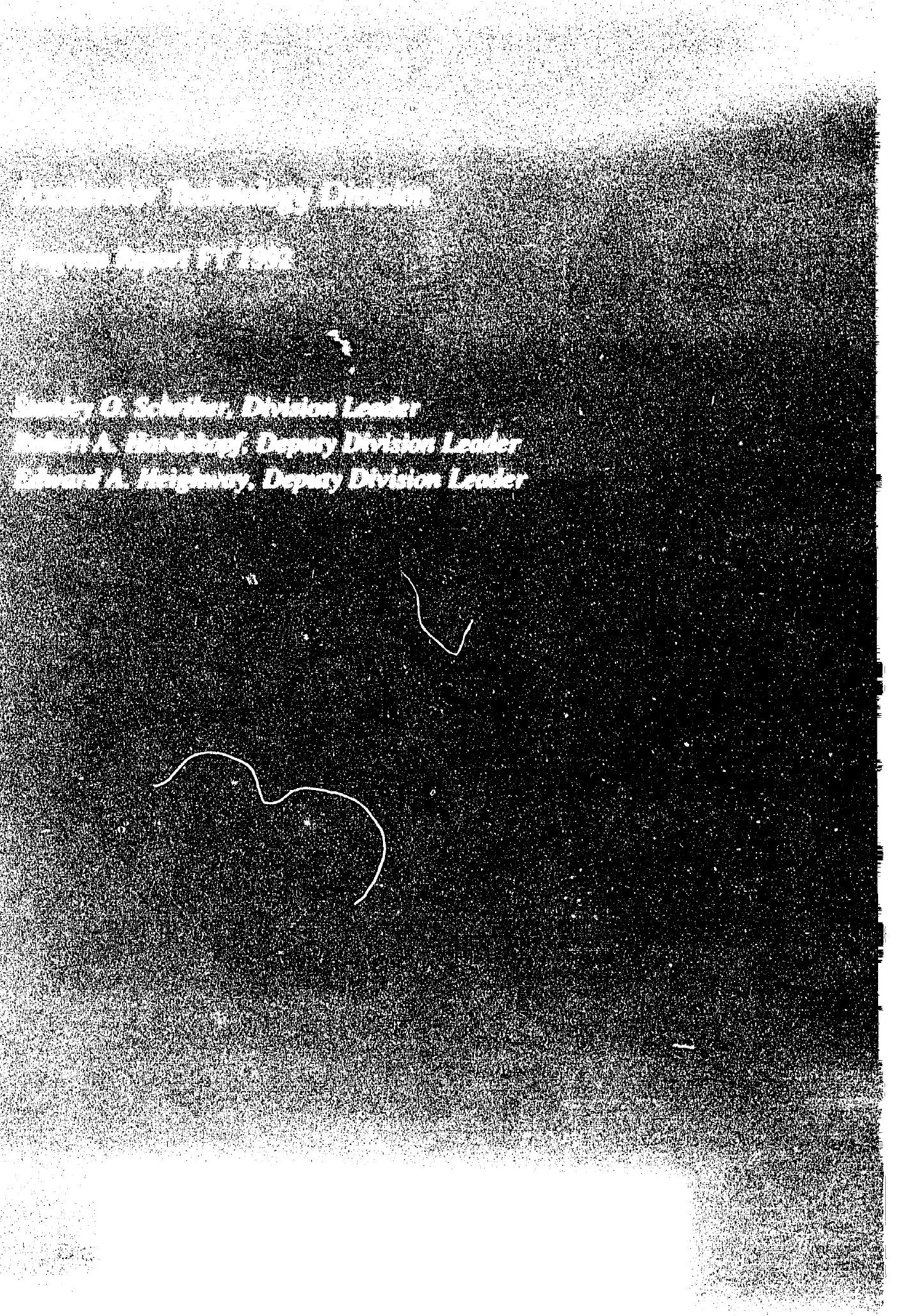




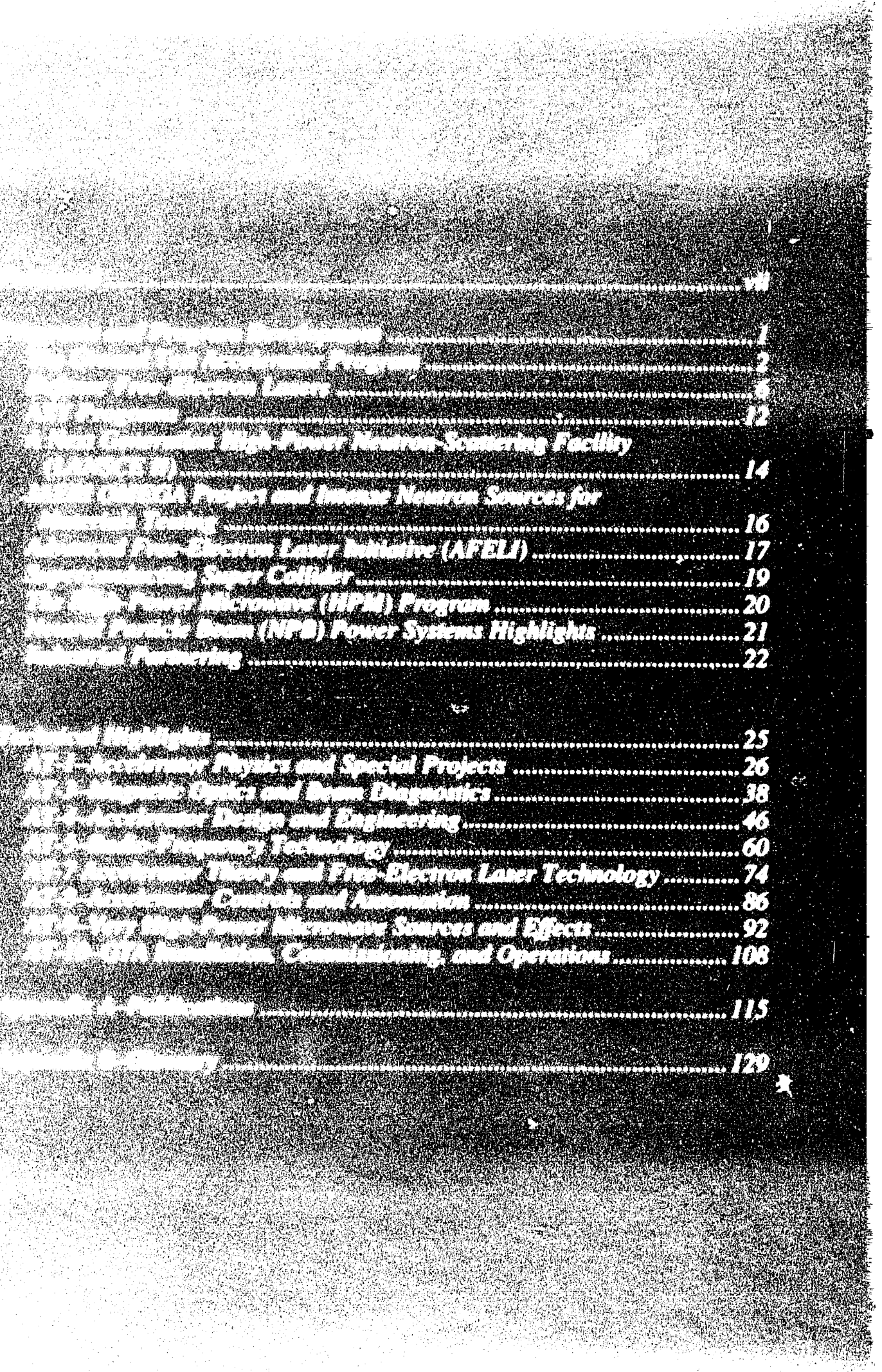




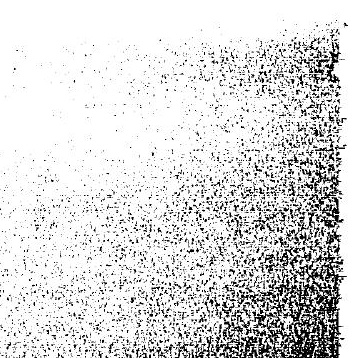

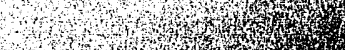

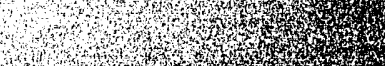

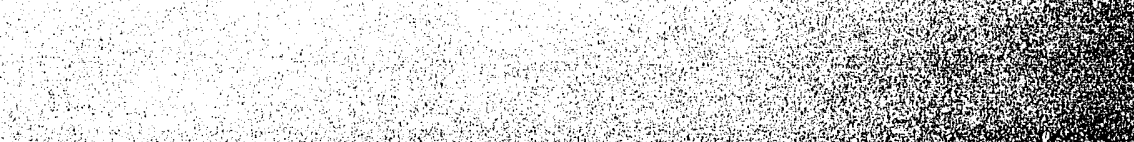

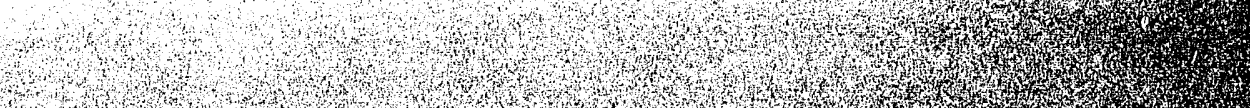
1
1

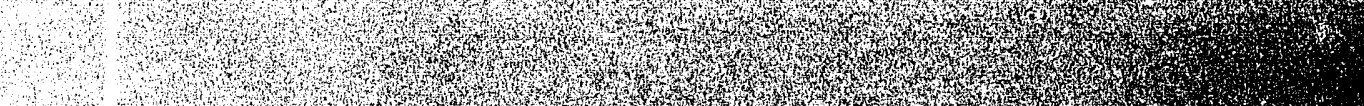

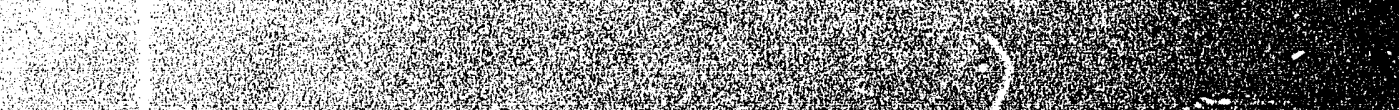

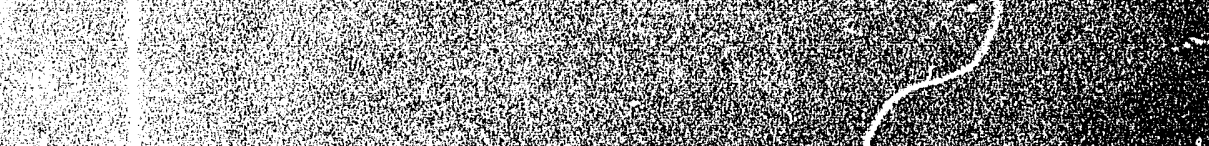

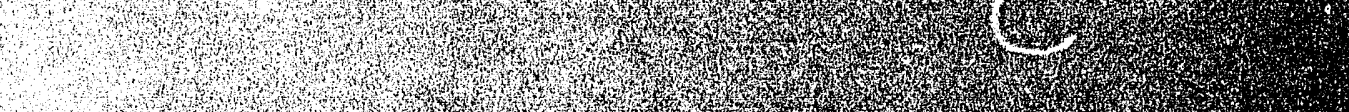
1
1

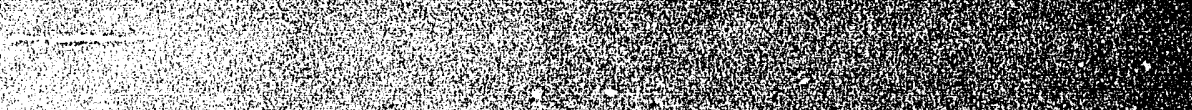

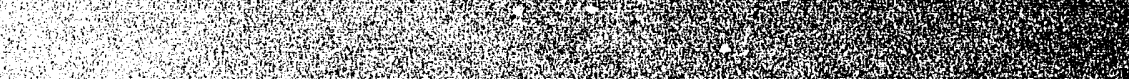

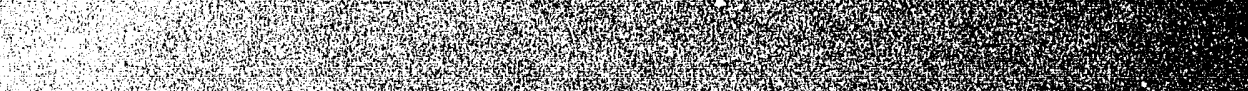

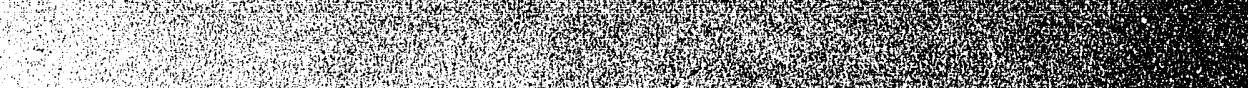

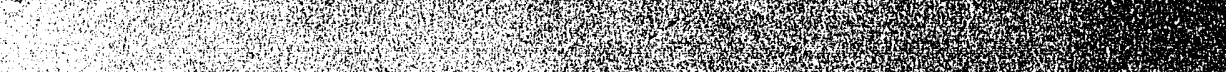

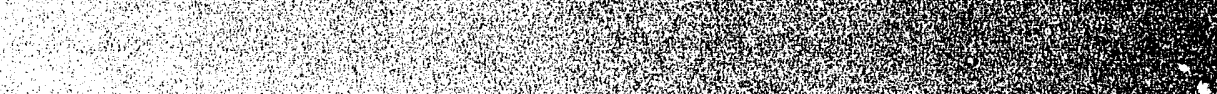

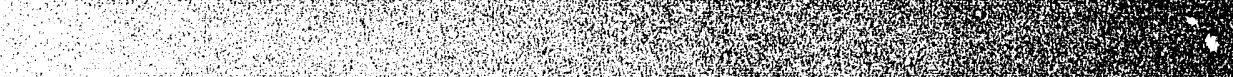

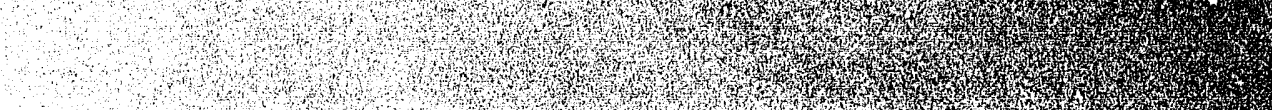
\begin{tabular}{ll}
-1 \\
\hline
\end{tabular} 
The Accelerator Technology (AT) Division continued its mission in fiscal year (FY) 1992 to pursue science and technology and to develop new accelerator concepts for research, defense. energy, industry, and other areas of nation al. interest. We had another success fuly $\mathrm{ear}$ with significant accomplishments in the Ground Test Accelerator (GTA) program, the Free- Election Laser (FEL) programs, the High Power 1 liorowave (HPM) program and miscellaneous other accelerator related projects. Highlights for the year included the following:

- The 6th AT Division Advisory meeting, June 30-July 2, 1992, attended by Dr. Richard Briggs, SSC Laboratory; Prof, J Mike Cornwall, UCLA; Dr, Robert Glidokstern, University Of Maryland, Dr. Hermann Grunder CEBAF, and Dr. Crowley Milling, England, provided good support and feedback in our activities and new initiatives.

- AT Division, Medium Energy Physics (MP) Division, Los Alamos Neutron Scattering Center (LANSCE), and other of ganizations, participated in the initial studies for an up graded LANSCE facility that would increase beam power to the target by at least an order of magnitude.

- All essential elements of the GTA accelerator have been tested successfully with bearn acceleration-including injector, radio-frequency quadrupole (RFQ), matching, drift tube linac (DTL), controls, if power, diagnostics, cryogenics, and beam design.

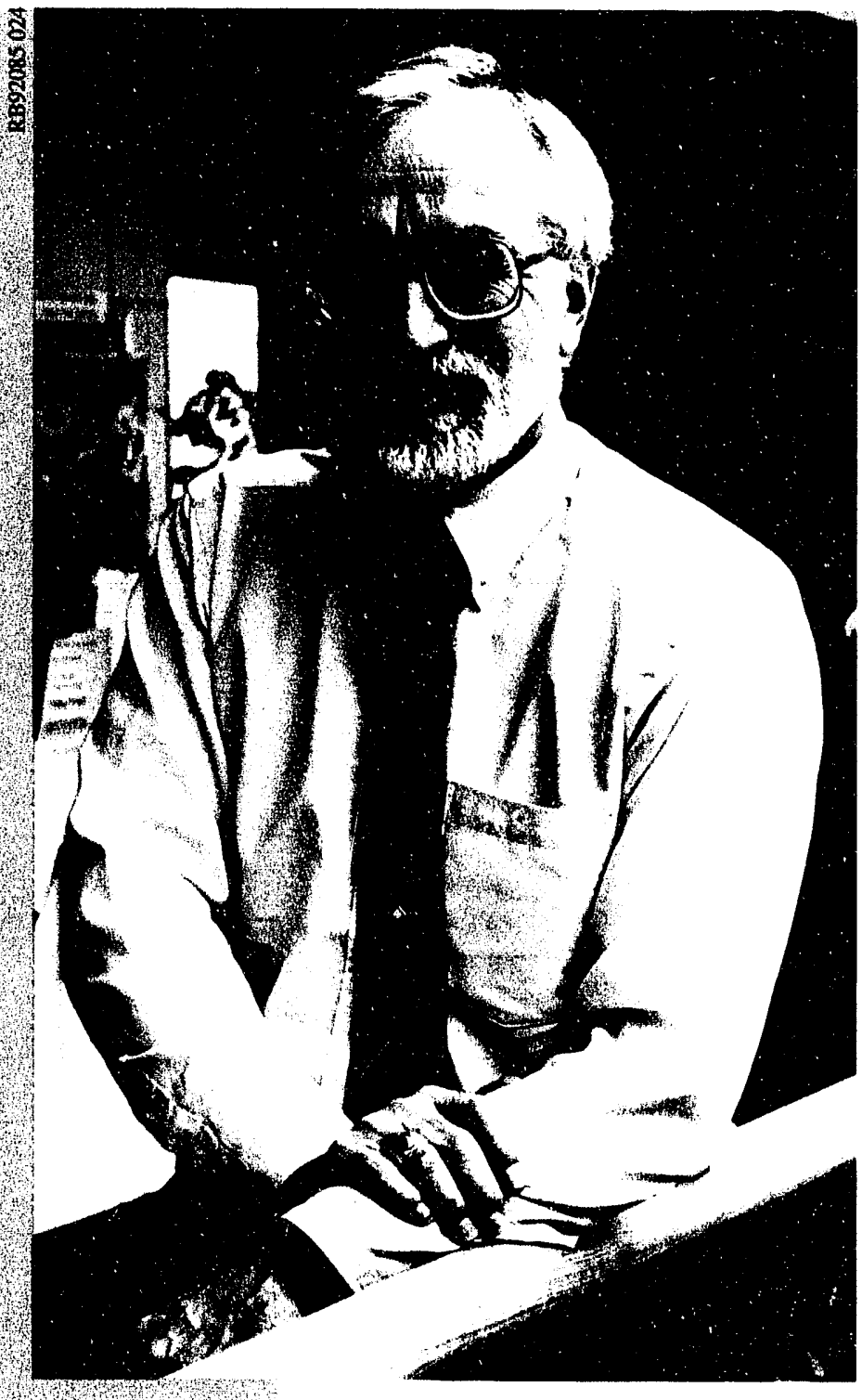

Stan Schriber, AT Division Leader

- The APEX facility and LANL staff continued to support the FEL program at Boeing with significant experiments and deliverables, including obtaining very bright beams through bends and small wiggler tubes, lasing at $837 \mathrm{~nm}$, and operating with a photocathode-based electron injector.

- Accelerator-based spallation neutron sources received much more attention; a number of potential applications were highlighted and new initiatives were started. These include APT, ATW, ABC, a front end cW demonstrator, and a new LAMPF/LANSCE possibility for neutron-based science/ technology studies. 


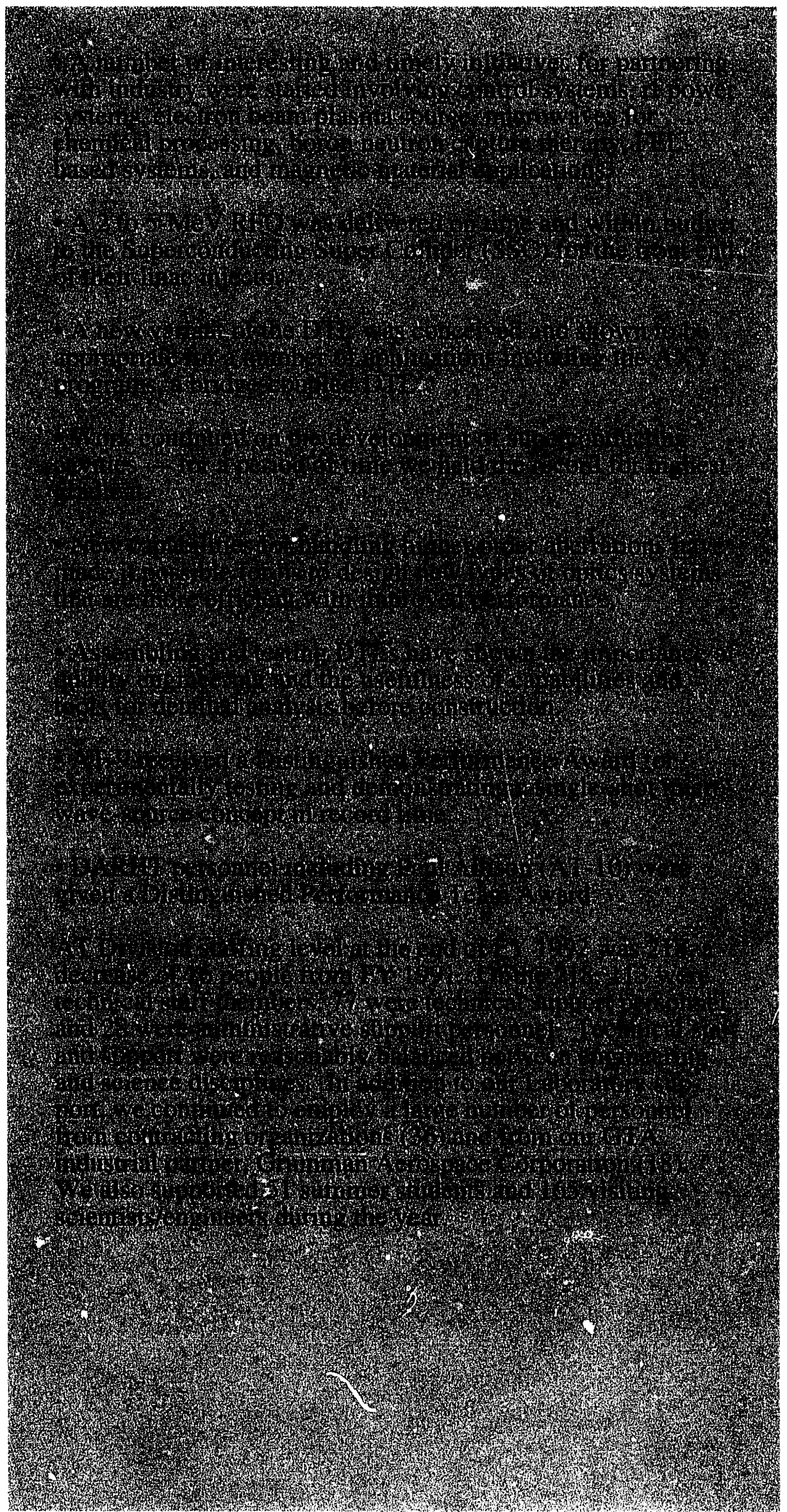


Programmatically AT-Division's funding level declined about $14 \%$ for FY 1992. As the chart on this page indicates, about $62 \%$ of the division was engaged in the GTA program. A strong effort in program development has continued to help diversify the division's activities. Security awareness was addressed using DOE and sponsor requirements. The AT Division Security Council keeps the division informed of new developments in security regarding education/awareness, visitors, and documentation. In July, efforts began to declassify much of GTA and the associated computing and physical security.

The DOE Tiger Team in late FY 1991 found no major problems in AT Division, however, valuable feedback on environment, safety, and health $(E S \& H)$ issues and conduct of operations was provided. The benefits of the inspection have resulted in an increased awareness of ES\&H and more efficient ways to accomplish ES\&H functions.

Tiger Team preparation and the formation of a directorate-based TA-53 ES\&H support team resulted in enhanced communication and cooperation between AT and other organizations at $\Gamma A-53$. Collaborative efforts have addressed site access control, emergency preparedness, common training issues, and accelerator interlock standards.

The division has taken steps to maintain ES\&H excellence in an environment of reduced funding. A formal self-assessment program, including quarterly inspections, was started and is coordinated by the division office; we are currently scheduling and coordinating ES\&H training. We are also performing waste management and inspection activities to reduce building manager workload. These initiatives are intended to reduce overhead costs and allow groups to invest more time in programmatic work. 
ES\&H continues to be a high priority Division opera tions were included in a Laboratory environmental compliance audit of TA 53, The GIA tryogenic coofing system passed important LAN and DOE safety reviews to allow acceptance testing with liquida hydrogen. A draf of the GTAPhase 2 (24 MeV) safety analy sis tepott was completed and sent to HS Division for internal we view. safety review of the Advanced Free eneetron 1 thes (AFEL) was conducted before any hazardous operations at the facility. The ALEL environnental assessment (EA) Was approved by DOE, and another E. ow w started for the phanned Accelerator Prototype Laboratom

Over 290 publications vere entered in the AT B vision

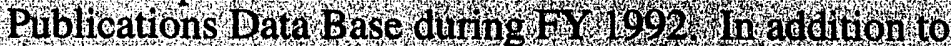
the publications, assistance was prov ded dor the produc ton of many proposals, reports, manvals and formins. The publications office also provided ex e e fise $10 \mathrm{computer}$ conversions. software ad viee, designand ta yout of news: letters, newsflashes, grapla, charts, vewg ga hs, and data bases.

In the following pages. we descrbe FY. 1992 progress with summaries of our majortegrams and Brogam

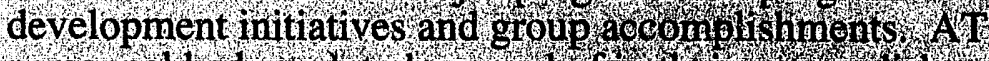
personnel had much to be proud of th the tracediptish ments:

I would like to take this opportunity to thank every ore in the division for their contributions, insights and d concerns and for sharing their many talents. Your efforts and youi striving for success have made the division stowger and have allowed us to be responsive to our many sponsoots. Thank you one and all.

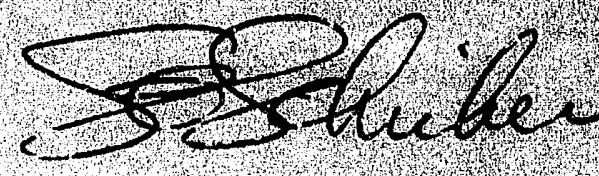

S.O. Schriber 


\section{Programs and Program Development}

The Ground Test Accelerator Program ........................................................... 2

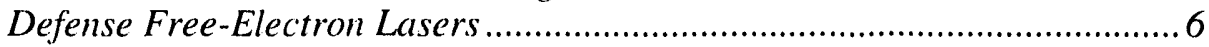

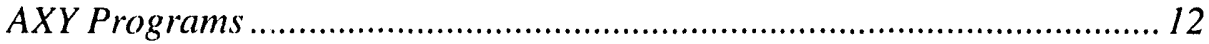

A Next Generation High-Power

Neutron-Scattering Facility (LANSCE II) .......................................... 14

JAERI OMEGA Project and Intense Neutron Sources for

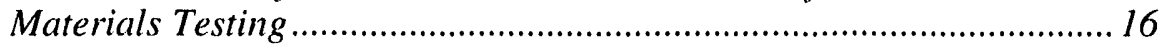

Advanced Free-Electron Laser Initiative (AFELI) .................................. 17

Superconducting Super Collider ............................................................ 19

The High-Power Microwave (HPM) Program ........................................... 20

Neutral Particle Beam (NPB) Power Systems Highlights.......................... 21

Industrial Partnering ............................................................................ 22

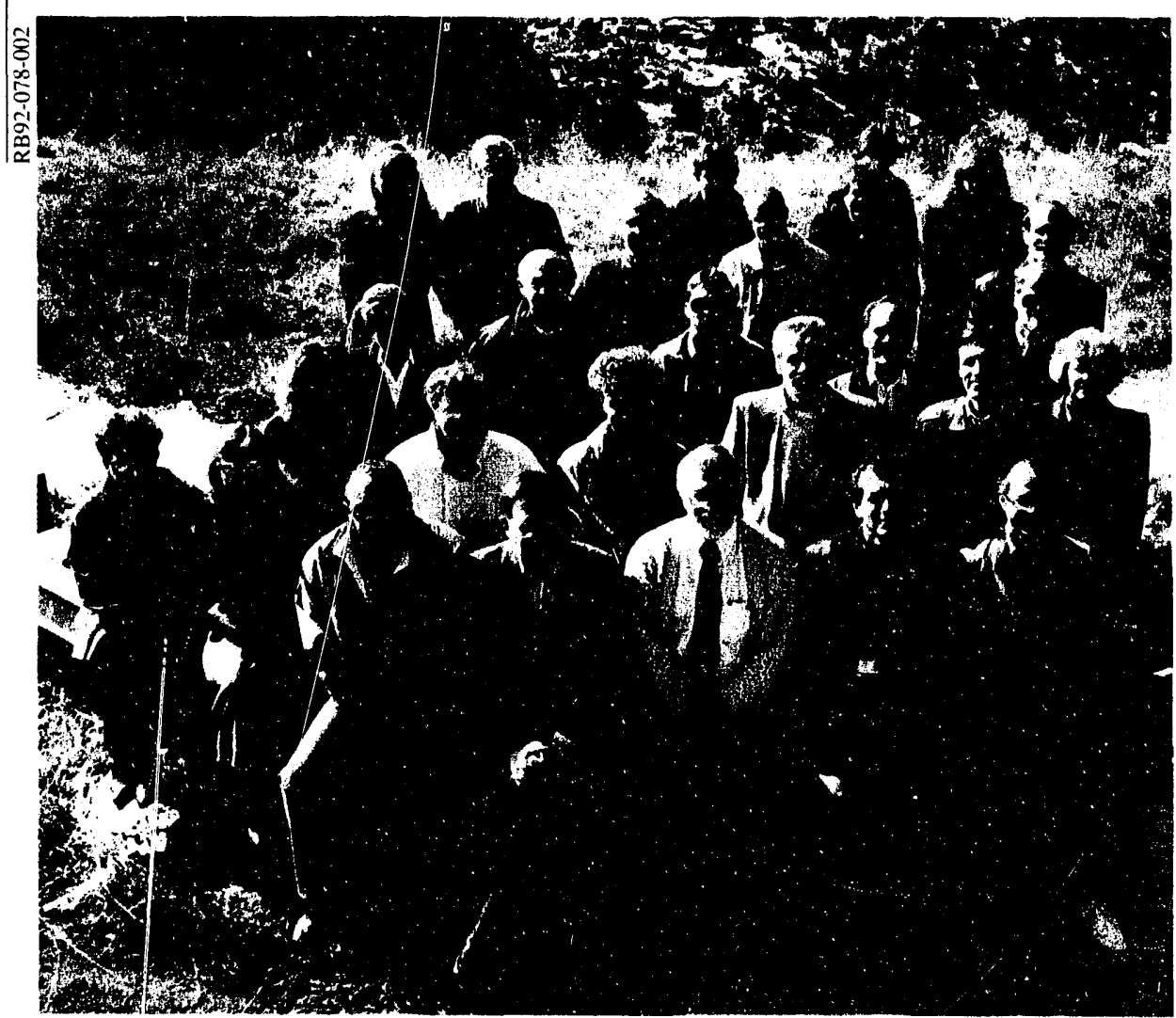




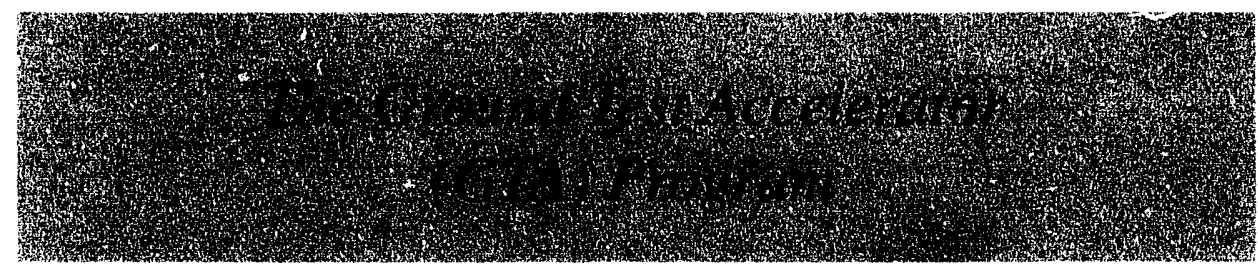

\section{Introduction}

Rapid progress continued throug, out this year with the following accomplishments. The GTA team procured and fabricated $\$ 12 \mathrm{M}$ of hardware. The matching section between the radiofrequency quadrupole (RFQ) and the drift tube linac (DTL) was installed on the beamline. Beam was accelerated. steered, and bunched for the matching to the DTL during a commissioning period from February to April. The first of the DTL modules was installed, and in a second commissioning run from September to November, beam was accelerated and steered through the module. As a result of these commissioning runs, we have now integrated and tested all essential elements of the Ground Test Accelerator (GTA): ion source, low-energy beam transport (LEBT). RFQ, intermediate matching section (IMS), one DTL module, three 425-MHz tetrode amplifiers. one 850$\mathrm{MH}$. klystron modulator, and the associated diagnostics and controls.

We continued to fabricate. assemble. and test off-line the DTL modules in parallel with our effort to fabricate. assemble, and integrate the radiofrequency (rf) power system. Utilities such as instrumentation and control (I\&C) and power wiring, water, air. vacuum, and cryogenic cooling were installed and made operational. The 4()-kW liquid hydrogen cr jo plant passed its acceptance test, and the GTA control room became fully operational.

Becaluse of at funding decrease from $\$ 50 \mathrm{M} 6 \$ 39 \mathrm{~N}$ we had to reduce GTA personnel from at alverage of 220) people in FY 1991 to an a rage of 150 people in FY 1992. Our industrial partnership with Grumman Acrospace continues successfully. with approxi- mately 18 Grumman engineers and scientists participating in all areas of GTA. We also employed 25 engineers. designers, and technicians from a number of manpower contract houses.

\section{Injector}

As a result of an off-line development, we were able to replace the small angle ion source (SAS) with a new $4 \mathrm{X}$ source. This source was designed with a $4 \mathrm{X}$ larger cathode spacing that decreased the power density on the clectrode surfaces. This replacement resulted in a very reliable operation and quieter currents of up to $65 \mathrm{~mA}$ with the expected design emittance. We have now accumulated about 3000 hours of successful injector operation.

To reduce the previously observed emittance growth in the LEBT, we shortened the length of the LEBT in two steps. First, we eliminated the steerer coils and used the physical motion of the ion source and the repositioning of the RFQ's upstre:m end for beam "steering." Second, we incorporated lambertson-type steering coils into the two solenoid lenses; steering with these coils was very satisfactory. The length of the LEBT was reduced by a factor of 2 from 150 $\mathrm{cm}$ to $75 \mathrm{~cm}$, thus reducing the observed emittance. We achieved required matching at the $R F Q$ entrance through optimization of two solenoid and two steerer currents.

In parallel with the operation of the $4 X$ source. a $4 X$ upgrade source was designed and fabricated. The improved features included a reduction of $\mathrm{H}_{2}$ flow by a factor of 2 to 3 , better precise dimensions after reassembly. and an enlarged cesium oven leading to mechanical tolerance for predictable $\sim 3(0)$ hours of operation. The reduced $\mathrm{H}_{2}$ gas flow lessens the need for pumping and thereby allows for a reduction in the length of the LEBT. Off-line tests of the $4 \mathrm{X}$ upgrade source are in progress.

\section{Radio Frequency Quadrupole (RFQ)}

The RFQ has operated very reliably with essentially no breakdowns during a succession of commissioning runs totaling $\sim 1000$ hours. Reconditioning required very short times ranging from 0.5 min during operation to $5 \mathrm{~min}$ after a night without operation. Reconditioning after exposure to air takes approximately 1 hour.

The RFQ was thoroughly evaluated against its performance specification and the physics design during experiment IB. To better understand the measured transmission we compared the original beam dynamics design with a new design that includes the effect of multipoles and image currents. This design has enabled us to understand the measured transmission.

\section{Intertank Matching Section (IMS)}

The IMS provides eight "knobs" to optimize the matching of the RFQ beam to the DTL entrance. Two cavities permit bunching, debunching, and acceleration. Four variable field quadrupole magnets provide for focusing and defocusing, and two quadrupole steering magnets permit steering and offsetting of the beam.

We had significant problems conditioning the IMS, particularly the convoluted coaxial feed line, because of multipactoring and electrical breakdowns. We solved the problem by reworking line details, heating a segment that ran in close proximity to the cold RFQ, and incorporating reflected if power protection. The IMS and feed line are now reliable; however, reconditioning time remains longer for the IMS than for the RFQ. 
The IMS was thoroughly evaluated during experiment $I C$. We measured centroid steering, transverse and longitudinal emittance, phase scans, and transmission. When we modeled the beam transport for low-brightness currents, we discovered the fixedfocusing strength of the quadrupole steering magnets had to be reduced. This reduction will result in a more "robust" behavior of the IMS. We plan to grind the aperture of the steering magnets to reduce the field strength.

\section{Drift Tube Linac (DTL)}

The two main thrusts in the DTL area included installing and commissioning the first DTL module on the beamline (experiment 2A) and fabricating, assembling, and off-line testing modules 2-10.

The first module was fully assembled; drift tubes were aligned at room temperature, and the module was tuned at room temperature. This included tuning the cavity to $850 \mathrm{MHz}$ and adjusting the couplers to achieve desired axial field distribution. We checked room temperature alignment and tune at the cryogenic operating temperature of $20 \mathrm{~K}$ in the low-power cryo test bed; DTL positioners were also exercised, and the position change of the DTL from room to cryo temperature was measured. Next, the module was conditioned up to $130 \%$ of the operating power at $20 \mathrm{~K}$ in the high- power cryo test bed. All 10 DTL modules will undergo the same test sequence before installation on the beamline. A picture of 8 DTL modules in various stages of completion is shown in Fig. 1.1.

The first module was installed on the beamline in vacuum vessel \#1 (the entire 24-MeV DTL will be housed in two vacuum vessels). It was fully integrated with diagnostics and controls and reconditioned in situ.

During experiment $2 \mathrm{~A}$, the first module was commissioned with the ion source, RFQ, and IMS, resulting in the most complete and successful GTA commissioning run thus far. The run demonstrated integrated operation of all elements of the 24-MeV accelerator. The purpose of experiment $2 \mathrm{~A}$ was to characterize the beam and the operation of the structures, to verify the design, and to prepare for beam transmission and acceleration through all 10 DTL modules.

The operation of the $3.2-\mathrm{MeV}$ accelerator (Fig. 1.2) was very reliable; all structures performed well. Reconditioning of the DTL module was rapid: $0.5 \mathrm{~min}$ without breaking the vacuum during operation, $10 \mathrm{~min}$ without breaking the vacuum overnight, and 1 hour after opening to air. The source delivered a very quiet, reliable beam. $V$ ast amounts of data were compiled as a result of the wide variety of measure- ments made on the following:

$R F Q$ matching and steering

$R F Q$, IMS, and DTL transmission

DTL transverse emittance versus

- macropulse time

- IMS variable field quadrupole times

- IMS buncher times

- DTL times

DTL longitudinal emittance vs input energy

Beam centroids vs steering (DTL, IMS)

Phase scans vs power (DTL, IMS)

$R F$ phase and amplitude control verification

Beam jitter

$X$-ray calibration of DTL power

Slit and collector vs microstrip probe

steering measurements

Hybrid LINDA tests

Video profile tests

\section{Radio Frequency (rf) Power}

During the DTL commissioning run (experiment 2A), all elements of the if power system were operated for the first time in an integrated mode. Three tetrode amplifiers provided reliable 425-MHz power to the RFQ ( $135 \mathrm{~kW})$, to the first IMS cavity $(6 \mathrm{~kW})$, and to the second IMS cavity $(12 \mathrm{~kW})$. One $850-\mathrm{MHz}$ dual-klystron modulator provided power for the first DTL module $(60 \mathrm{~kW})$ and the high-power conditioning stand. The system was very responsive to the daily needs of the commissioning team and worked reliably.

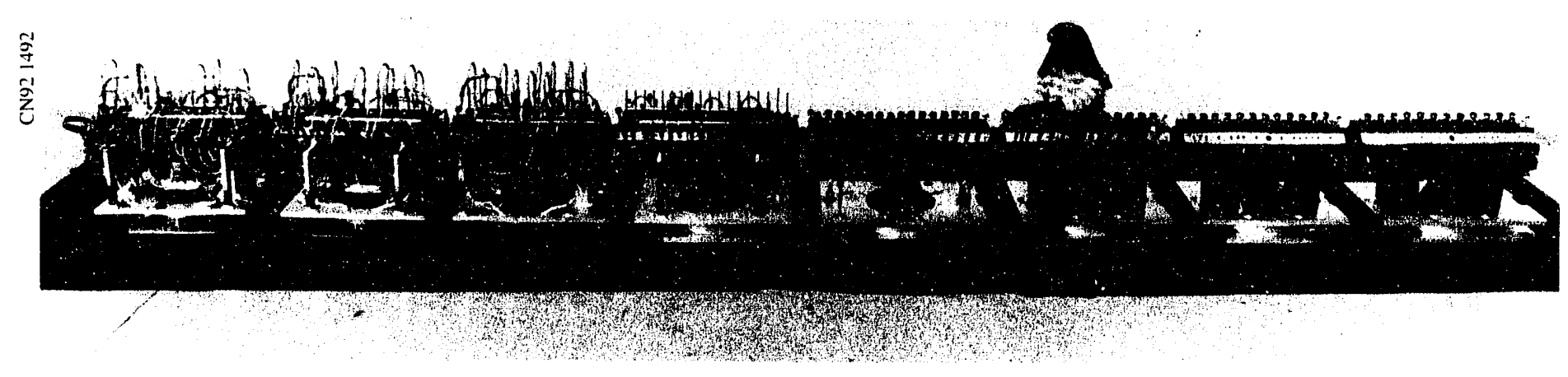

Fig. 1.1. Drift tube linac module satus (Juanita Romero. AT-4). Production line in full operationi fire modules complete. 


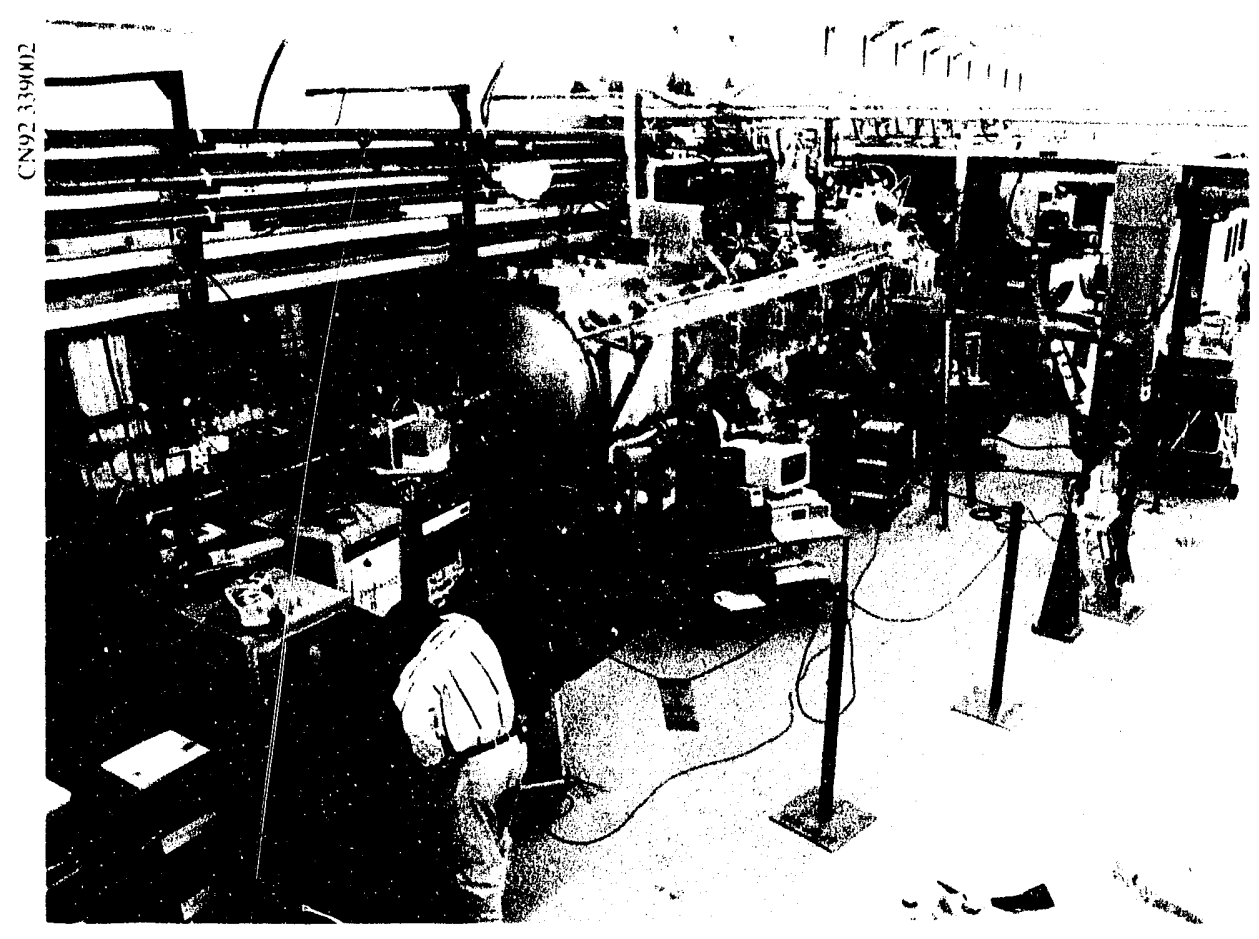

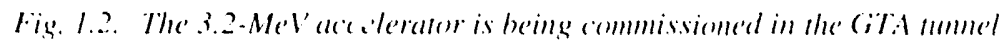
left. Darryl Sandenal and Ralph (iarcia, AT-i).

The off-line testing of the 12 Thompson klystrons a as completed successfully. The second modulator has been assembled and checked out on the mes/anine. The remaining modulators are in various stages of assembly (Fig. 1.3). The large capacitor bank used for creating the 2-ms pulse has been assembled and is ready for integration with the crowbatr system and the $5-1$ high-voltage power supply. The lowlevel af control system has been designed. and circuit hourd production for the prototype has begun.

\section{Beam Diagnostics}

All beam diagnostics necessiry for diagnosing and controlling the beam hare been integrated inte the alceelerator and thoroughly exercised. These include an attomated emiltance scanner used to measure beam emittance in the LEBT. Toroids, hefore and after the RFQ and the DTI. are used to meatsure beam current and transmission. Microstrip probes lecated in the IMS and on the diagnostic plate measure beam centroid position. angle, and energy. A viden profile system after the IMS measures ecentroid profile and position. Two slit and collector assemblies and three Faradily cups on the diagnostics plate complete the heam diagnostics for the 3.2-MeV arcelerator.

The beam diagnostic's worked well during experiment 2A, although questions remain regarding centroid position and angle measurements of the large aperture microstrip probes used on the diagnostics plate. Redesign of the beim tube for beller continuily and symmetry of the return currents should correct this deficiency

We dereloped and used suecessfully a fist-protect module batsed on the transmission loss through the RI:Q and DTl. which is measured ats a current difference. We also developed and performed off-line kests on a prototype beam-loss monitor batsed on $\gamma$-raly detection. These beam-loss monitors will be used throughout the alcelerator at higher energies.

\section{Controls}

The controls systems ti.c.. system softwate, application software, and the If (s) have kept palec with the demands of the growing (iTA liacility. These systems exhibited good reliability during the last commissioning run (experiment 2A) and enabled the commissioners fo collect, archive, and retrieve data yuickly and reliably. Several diagnostic processer steh ats cmillance scimning and phase seamming have been atutomated. They mork well and hate redued drambatically the time required for data alcyuisition.

Recently during experiment 2A, we introduced several new control funclioms and demomstrated them stecessfully. These include the list-proted function. the alarm manager, and an adaptive leed-forward module that controls phase and amplitude fluctuattions of the rf cavity power. The control room was made fully operational and is working satisfactorily.

\section{Cryogenic Cooling Plant}

A 4 ()-kW licyuid hydrogen plant was designed and built during the last 3 years. This plant uses liquid hydrogen as the primary condant for a highpressure gascous helium loxp hat actually cools the accelerator cavities to 20 K. ()ne of the most difficult and time consuming tasks involied preparing the Salfety Analysis Repont and receiving final approval from the health and salfely organizations of the L aboratory and Department of Energy. which look 3 years and severat manyears of effort to acomplish. Moreover, the eryogenic plant passed its acceptance lest in May and is now fully operational. The $4($ )-kW plant will be used fo cont the entire 24-MeV accelerator in $\mathrm{F} Y \mathrm{Y} 1094$.

We continue lo employ the existing $7(0)-W$ Koch refrigerator for all experiments with the existing heamline up to $3.2 \mathrm{MeV}$ and tor the high power conditioning of all 1'Tl. modules.

\section{Optics}

The (iTA optics keam continued an support the mentral particle heam spalce experiment (NPBSE) design effort led by Grumman. Several designs were produced. 
The design for the ground based optics for GTA progressed successfully and culminated in a physics design for the high-energy beam transport (HEBT) section the $180^{\circ}$ bend. and a conceptual physics design for the telescope. The key parameters for the optics design are

-3.1-m diameter bend.

- II-m long, 12.5-cm diameter. telescope.

- 5th order aberration control, and

- 6 urad divergence.

The purpose of the HEBT is to match the DTL to the bend and provide for longitudinal expansion and beam steering into the bend, which is accomplished with four variable field permanent-magnet quadrupoles while providing for a dejitter cavity if necessary.

The bend's purpose is to demonstrate the feasibility of $180^{\circ}$ bending of highcurrent beams with minimal emittance growth and beam loss. The bend accomplishes this with a triple achromat, each containing five cells with a defocusing, bending, and focusing permanent magnet. The bend is designed to transmit currents up to $50 \mathrm{~mA}$.

A major breakthrough was achieved during FY 1992. We developed a method to contour high-order field components to minimize the feed-up of geometric aberrations. The contoured high-order components were built into the permanent-magnet objectives by tailoring the radial and rotational positions of the permanent-magnet stacks. Electromagnetic corrector packages for second through sixth order correction permit the correction of effects due to geometrics, ambient fields, and space charge. By using this method, we can reduce the telescope length to $11 \mathrm{~m}$. To minimize the cost of the new objectives, we will reuse the permanent magnets from the original $40-\mathrm{m}$ telescope objectives.

\section{Summary}

FY 1992 was very exciting technically. It culminated with the $3.2-\mathrm{MeV}$ commissioning run that demonstrated the integrated operation of all elements of the 24-MeV accelerator. The accelerator ran successfully during 3 weeks of experimentation, the beain current was quiet and stable, the if power system delivered power reliably, and the cavities performed withour electrical breakdowns. Our commissioning team will be busy for months evaluating the large amount of data acquired.

Our plans for FY 1993 are to install all 10 DTL modules into 2 large vacuum vessels over the waveguide basement. In parallel with this activity, we will complete the 5 dual-klystron modulators and build 1 preproduction lowlevel if control system. Because of severe funding constraints, we will bring the optics designs only to the preliminary design review level without making any long-lead procurements.

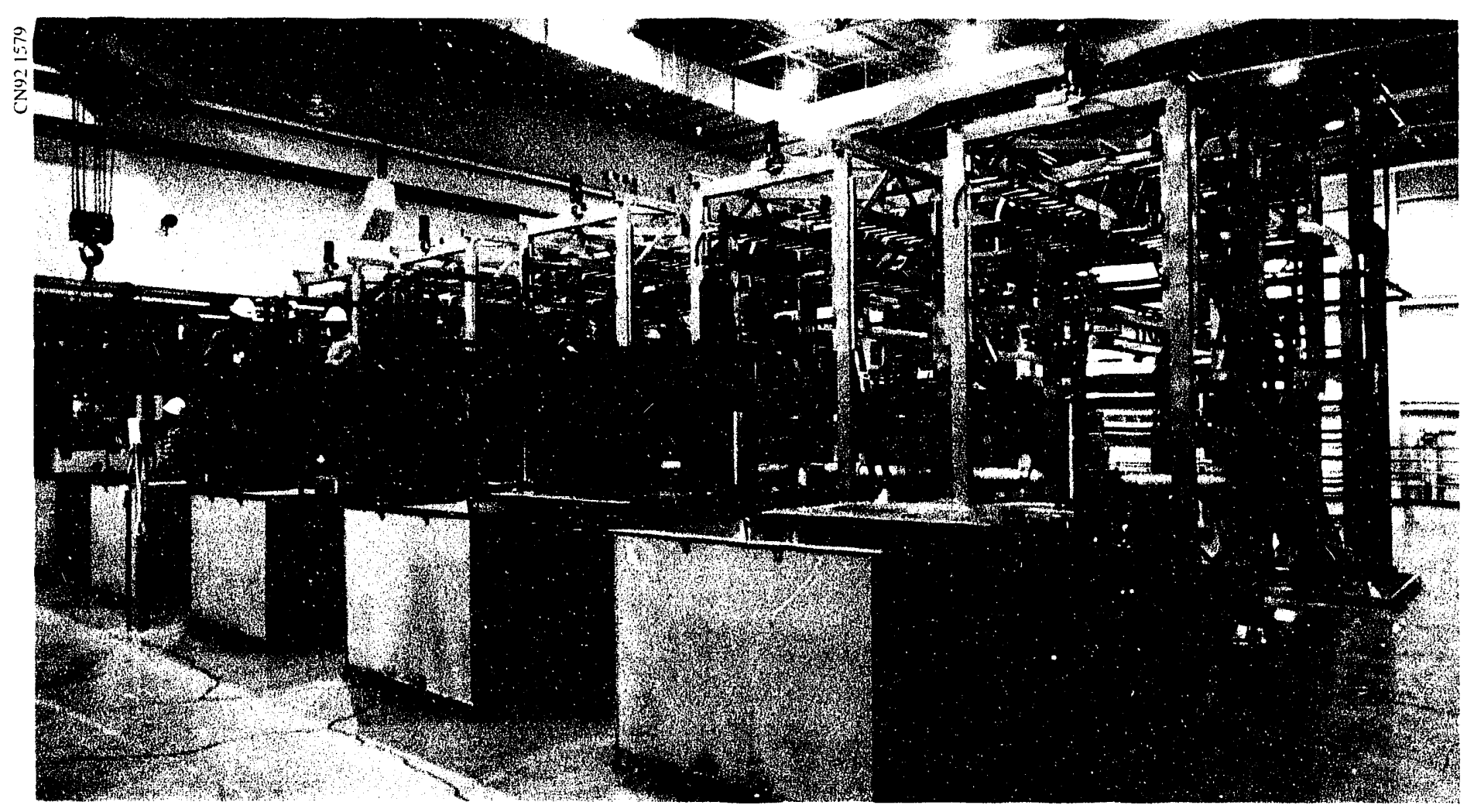

Fig. 1.3. Bill North (left), John Bancroft, Bill Reass, and Glen Zimmerman prepare five high-power klystron modulators for operation. 


\section{Introduction}

During 1992 the Los Alamos Defense Frec-Electron Laser (FEL) Program continued to he teamed with Boeing Aeruspace and Electronics in support of the Alerage Powe Latser Experiment (APLE). The ultimate aim of the APLE program is 10 demos.strate that a FEL can produce laser light with an aserage power of $100 \mathrm{~kW}$. A major Los Alamos contribution to this effort is the APLE Prototype EXperiment (APEX). The purpose of APEX is to demonstrate the basic physics and technology of APLE at low-duty tactor. Following the 194) commissioning of APEX. the 1992 effort focused on physics experiments associated w ith the FEL operat- tion. Highlights included lasing at a wavelength of $8.37 \mathrm{~mm}$, the shortest to date for a Los Alamos FEL. Ultrahigh current densities of $70(0) \mathrm{A} / \mathrm{cm}^{2}$ were demonstrated from the APEX photocathode, significantly exceeding the previously reported values for multialkali photocathodes. The electron beam's brightness at the end of the APEX 4()-MeV accelerator was measured to be $3 \times 10^{\prime 2} \mathrm{~A} /\left(\mathrm{m}-\mathrm{rad}^{2}\right)^{2}$ at a current of $13.5 \mathrm{~A}$ making the APEX heam one of the world's brightest highcurrent electron beams. Single-bunch wakefield effects were mealsured directly for the first time using fast streak camera techniques, and a scheme for mitigating wakefield effects in a high-current accelerator was developed
In addition to supporting the APLE program, the APEX facility was used to support a DOE-funded experiment that demonstrated the photolithography of semiconductor computer chips using a FEL beam. At the Boeing facility in Seattle, the joint Boeing/Los Alamos high-duty 5-MeV-photoinjector test stand achieved operation at $25 \%$ duty factor and an average electron-beam power of $160 \mathrm{~kW}$. Such high-duty operation of a photoinjector was an important milestone on the road to the high-power FEL goal.

The APLE program involves many Los Alamos National Laboratory groups: APLE Project Office (program management), AT-7 (accelerator physics and APEX project leadership), CLS-6 (drive-laser and optical diagnostics), P-15 (electron beam diagnostics), MST-7 (photocathodes), X-1 (theory and simulation), AT-4 (mechanical engineering), AT-5 ( $r f$ controls), and AT-8 (automation).

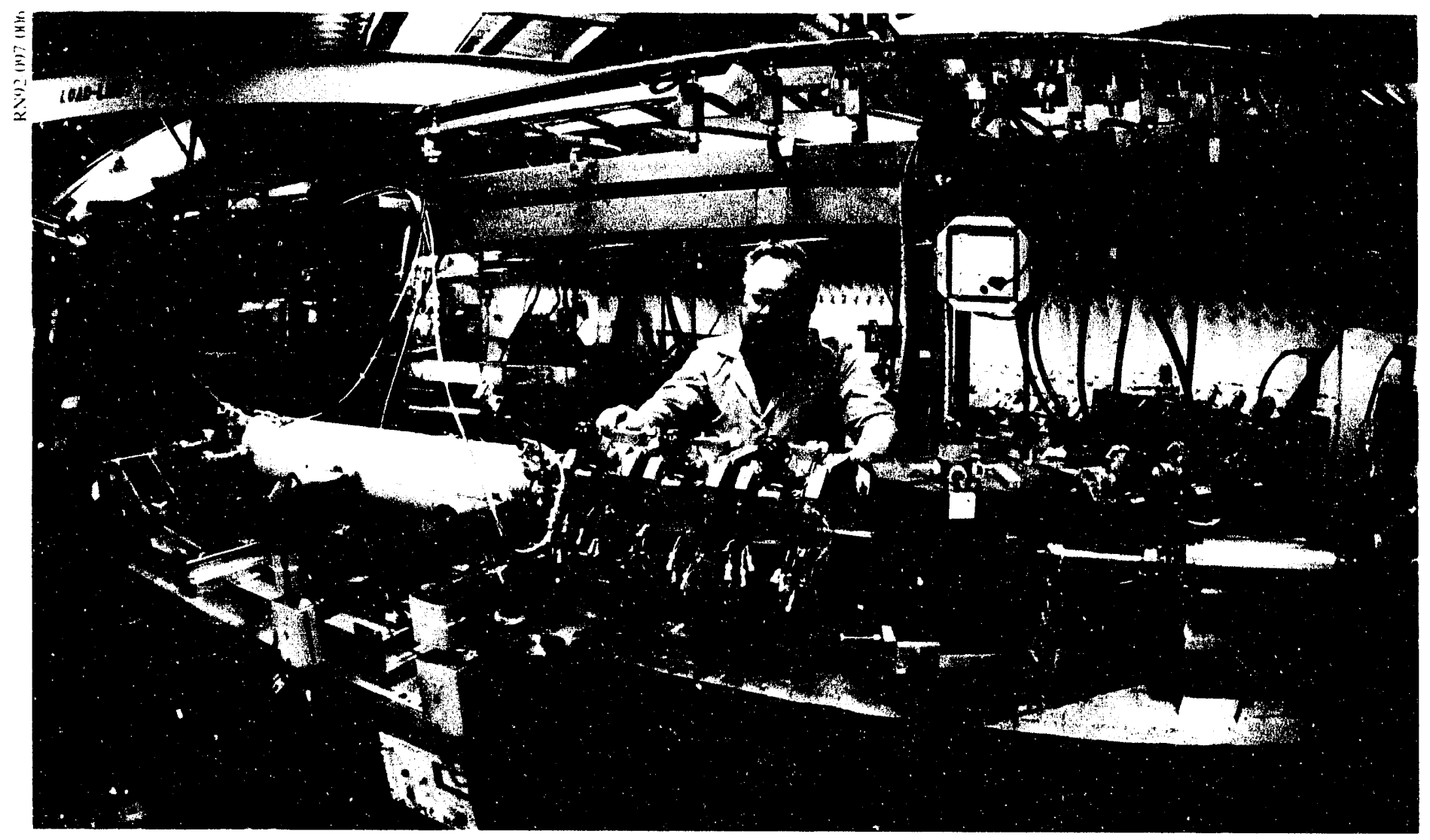

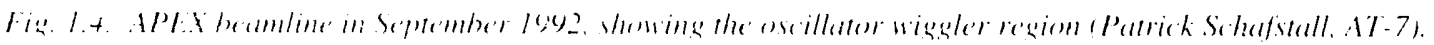




\section{Background}

Funding for the Los Alamos Defense FEL Program is provided by the Strategic Defense Initiative Organization (SDIO) and the United States Army Strategic Defense Command (USASDC). Under the auspices of SDIO, a collaboration on FEL research was initiated between Los Alamos and the French Atomic Energy Commission FEL facility at Bruyère-le-Châtel near Paris. The French are in the process of commissioning a FEL driven by a radio frequency $(\mathrm{rf}$ ) photoinjector linac similar to that at APEX. During FY 1992, a number of mutually beneficial exchange visits were made to both FEL facilities.

Following the initial lasing of the APEX FEL in June 1991, we directed our efforts in FY 1992 toward characterizing the electron and optical beams with the goal of verifying the design codes used for the APLE FEL design. A secondary goal was to complete the APEX beamline through the 150)degree bend and FEL amplifier leg. A new effort was undertaken to bring the sections of the APEX beamline under automated computer control. These goals were achieved as planned.

\section{Achievements}

During FY 1992, the APEX linear accelerator operated in a hitherto unexplored regime of high current and high charge $(>100 \mathrm{~A}$ at $1 \mathrm{nC}$ ) and low emittance $(<5 \pi \mathrm{mm}$-mrad normalized, rms).' The 40-MeV linac has four separately driven tanks; the first is a 6 $\mathrm{MeV}$ on-axis coupled structure, and the remaining three are side coupled. All the structures operate in the $\pi / 2$ mode at $1.3 \mathrm{GHz}$. The peak current is approximately $300 \mathrm{~A}$ in 15-ps fullwidth-al-half-maximum (FWHM) bunches. Individual bunches are separated by $46 \mathrm{~ns}$. This bunch spacing is determined by the round trip time for the optical pulse in the FEL resonator. The FEL configuration used for our experiments was an oscillator with a near-concentric resonator and an untapered permanent-magnet wiggler in the Halbach configuration.

Detailed descriptions of the APEX accelerator and FEL can be found in Refs. 2-5. Figure 1.4 shows the oscillator wiggler and adjacent sections of beamline.

\section{Photoelectron Emission}

The photocathode material used at APEX is $\mathrm{CsK}_{2} \mathrm{Sb}$ driven by a frequency-doubled $\mathrm{Nd}$ :YLF laser." We chose this configuration because it produces high electron-beam current for modest drive-laser power. We studied the photoe' dission current as a function of both drivelaser power density on the cathode and injection phase relative to the rf. Figure 1.5 shows the photoemission characteristic curves for our cathode. In generating these curves, we held the cathode's diameter at a constant $3 \mathrm{~mm}$ and the drive-laser pulse length at $10 \mathrm{ps}$. For each drive-laser power, we varied the injection phase and measured the charge extracted using a wall-current monitor immediately downstream from the photoinjector, approximately $80 \mathrm{~cm}$ from the photocathode surfice.

The electron current at the cathode surface is not measured directly. Because the electron emission from the cathode is prompt, the temporal length of the electron pulse near the cathode is less than or equal to the drive-laser pulse length. We measured the charge for each micropulse reaching the wallcurrent monitor. The current at the cathode is estimated by dividing the total charge per micropulse by the width of the drive-laser's FWHM pulse.

The variation of drive-laser phase and power have their analogs in thermionic cathodes. Phase is analogous to the cathode voltage, and power is analogous to cathode temperature. (Note that increasing the drive-laser power does not increase the emission energy of an electron; it increases only the number of electrons emitted.) Figure 1.5 illustrates the source-limited regime (low drive-laser power) and the spacecharge-limited regime (high drive-laser power). At low drive-laser power, the

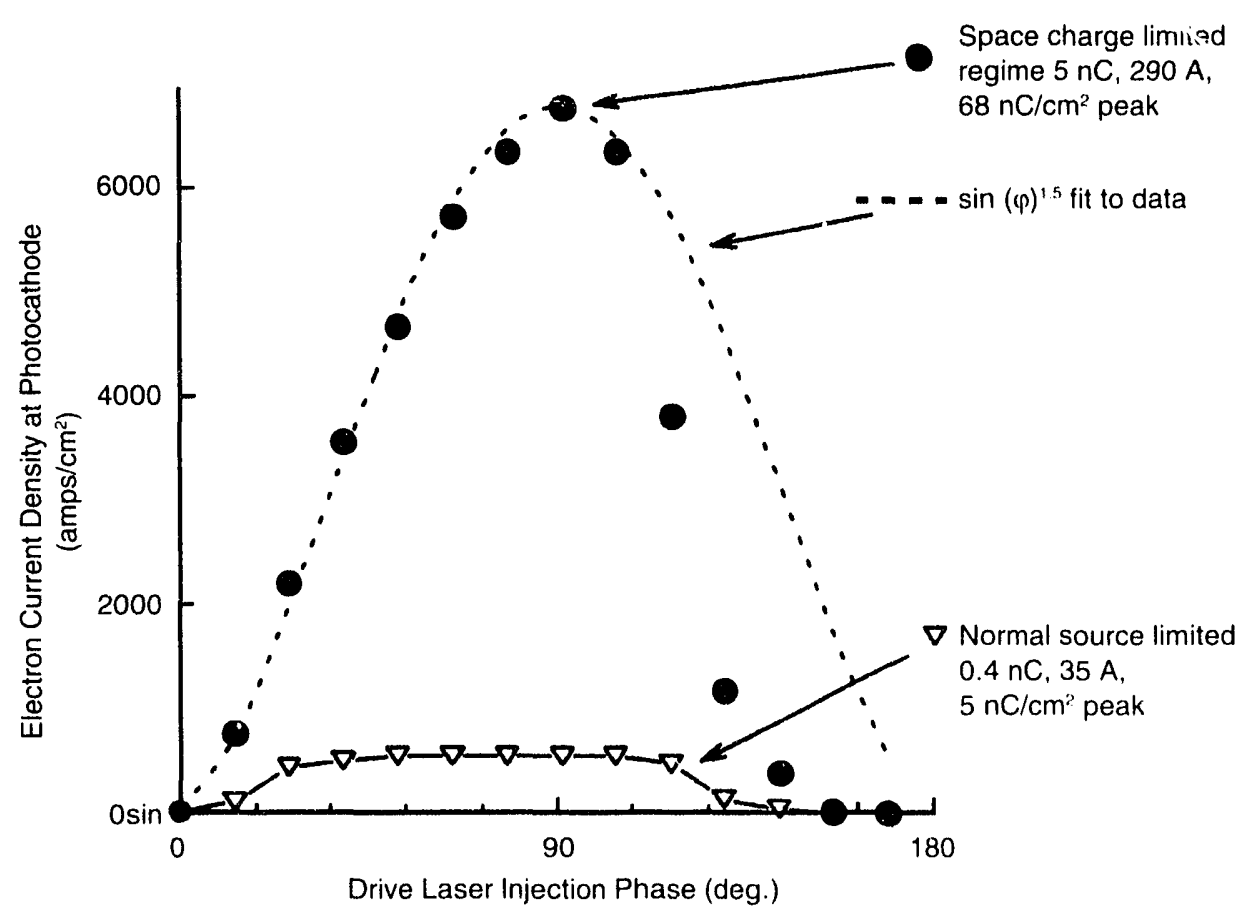

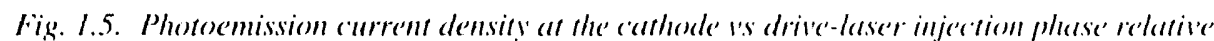

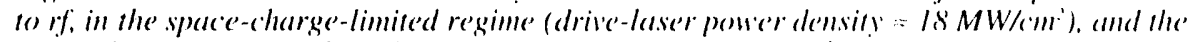

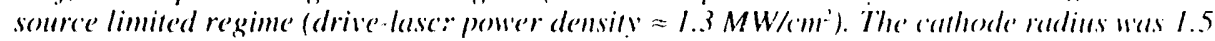
mm and the drive-laser pulse width weas $10-p s$ FWHM. 
curve makes the transition from spacecharge-limited emission (()" to 2(1) to the source-limited emission (flatt top) and then back to space-charge limited again. This corresponds to the normal operating drive-laser power and current density at APEX.

At high drive-laser power $\left(18 \mathrm{MW} / \mathrm{cm}^{2}\right.$ or $18 \mu \mathrm{J} / \mathrm{cm}^{2}$ per micropulse), the emission is always in the space-chargelimited regime. In fact, we have observed current densities as high as $6800 \mathrm{~A} / \mathrm{cm}^{2}$. The corresponding charge emitted per micropulse is $68 \mathrm{nC} / \mathrm{cm}^{2}$. For a nominal cathode diameter of 10 $\mathrm{mm}$, an extracted charge of $50 \mathrm{nC}$ per micropulse would be expected for the equivalent drive-laser power density. This regime is far from our normal operation. Becaluse our accelerator is not designed to effectively transport such high charges and currents. we anticipate that significant emittance growth will occur. However, these results demonstrate the capability of photocathode systems to produce very high currents.

\section{Emittance and Brightness}

Following the photoinjector. the beam is accelerated (o) approximately 40 MeV by three side-coupled $\pi / 2$ standing wave structures referred to as lanks B, C. and D. Each lank is separately powered by a Thomson TH20195 klystron. Between each tank we have inserlable view screens from which the electron-beam profiles ate imaged using optical-tansition radiation (OTR). Electron-beam micropulse current is determined from wall-current-charge monitors and O'TR streak-camera micropulse measurements.

Fmittance is measured at the end of the linac using the quadrupole-scan technique. The emittance numbers yusted here are normalized root-meansquare values. Figure 1.6 shows the emittance ats a function of micropulse current measured at an electron-heam energy of $36 \mathrm{MeV}$. The numbers plotled are the geometric mean of iwo orthogonal measurements averaged over $4+1)$ micropulses. The predicted curve is generated using the code PARMEL.A. the primary beamdynamics code used for electron-linac design at Los Alamos.

The ammormaliaed emillance at $13.5 \mathrm{~A}$ is $0.046 \pi \mathrm{mm}-\mathrm{mrad}$, which is sulficient for FEL lasing at wavelengths as short as $160 \mathrm{~nm}$. The measurement of such low emittances at high charge and current from a photoinjector linac verifies the solenoidal emittance compensation scheme proposed by Carlsten.:

Figure 1.4 shows the corresponding normalized rms brightness as a function of current. The brightness is defined ats

$$
\mathrm{B}=2 \mathrm{I} / \varepsilon^{2} \text {, }
$$

where $I$ is the micropulse current and $\varepsilon$ is the rms emittance. The factor of $\pi$ in the definition of emittance is explicitly used to determine the brightness. The maximum brightness observed to date is $3 \times 10^{13} \mathrm{~A} /(\mathrm{m}-\mathrm{rad})^{2}$. at a current of 1.3.5 A and an electron-beam entergy of $36 \mathrm{MeV}$. The energy spread, which was averaged over 220 micropulses. was meatsured at $0.24 \%$ FWHM. This measurement compares with at PARMELA prediction of (). 1.5\% for a single micropulse.

\section{Transverse Wakefields}

We halle paid particular altention to mechanisms that degrade the emittance and brightness of the electron beam. During FY 1992. we focused on the effects of transwerse wakefieds in the linac. Becatuse of dipole if fields in the side-coupled accelerator tanks, transverse kicks are experienced in the electron heam that induce head-to-tail transwerse-wakefield kicks in the micropulse and hence result in emiltance growth. The trajectory required to minimize the wakelields is not intuitively obvious to the accelerator operator. To assist in determining the optimum trajectory. we installed our streak camera to monitor the electron micropulse temporal and spatial profile on a sereen focated at the end of tank D. Consequently, we were able to directly observe the eflects of the watefields on the electron heam as shown in ligg. 1.7. The operator can easily choose a trajectory that minimizes the wakefields and optimizes the emittance. A detailed knowledge of the optimum trajectory is not recuired. The degree of emittance degradation resulting from watefiedds is shown in Fig. 1.6. In this figure. the PARMLI.A curve withoul wakeficlds corresponds to the emiltance when the clectron beam is steered to minimize wakefiedd effects. The PARMLI.A curve with wakefields corresponds to the electron beam when it is stecred to center on the screens between each acceleratur lank.

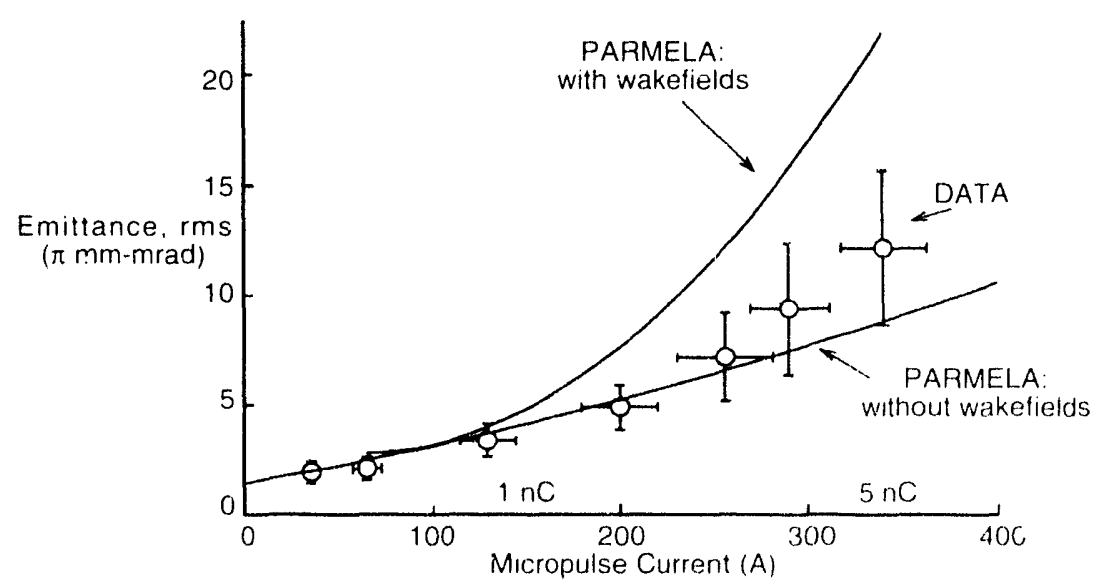

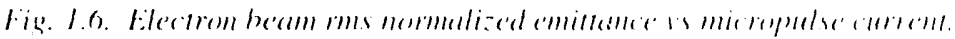




\section{FEL Performance}

The detailed specifications of the APEX FEL resonator and wigglers are given in Ref. 5. Typically, lasing has been at wavelengths near $3 \mu \mathrm{m}$. The 6.9-m resonator is an asymmetric, nearconcentric design. The asymmetry results from placing the optical waist at the wiggler center, which is $0.5 \mathrm{~m}$ upstream from the resonator's geometric center. The resonator mirrors are coated with a multilayer dielectric to reflect greater than $99 \%$ at $3.0 \mu \mathrm{m}$. A small fraction of light, which is transmitted through the optic, is transported to a diagnostic table located within $5 \mathrm{~m}$ of the out-coupler. Sensors on this table allow for characterization of the small-signal gain, cavity loss, energy, spectral content, spatial intensity distribution, and temporal intensity evolution.

The resonator mirrors tend to suffer coating damage when the macropulseaverage intracavity mirror flux is high. Consequently, the macropulses are kept short (20-40 $\mu$ s). and the charge is kept low ( I $\mathrm{nC}$ ). During the lasing described below, the electron-beam micropulse is usually lo-ps FWHM, with a macropulse energy spread of less than $0.5 \%$ and a normalized rms emittance of approximately $4 \pi$ mm-mrad. Simulations with the above configura-

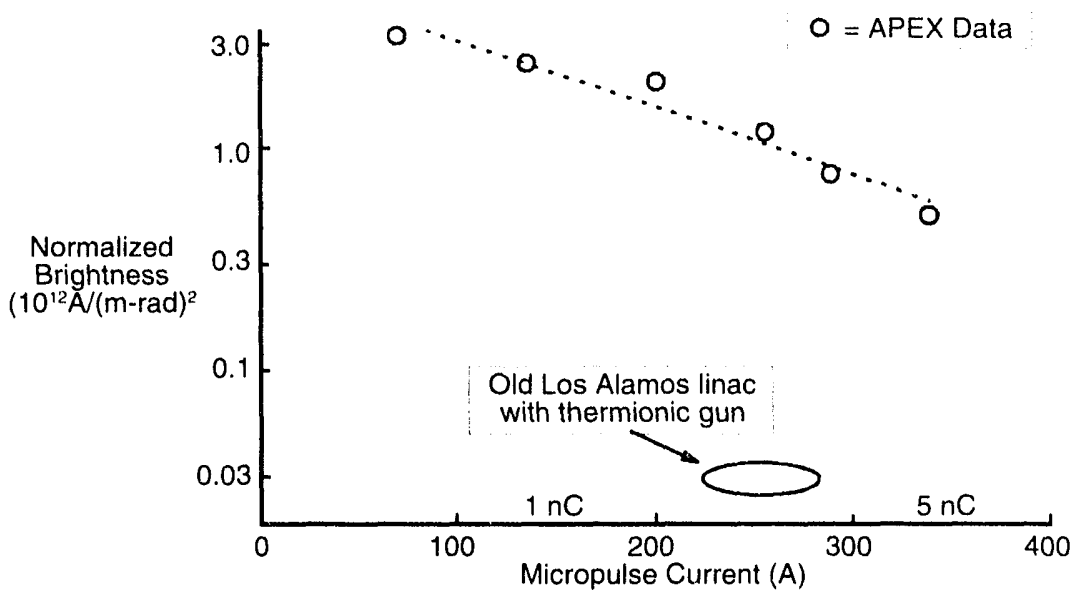

Fig. 1.7. Electron beam rms normalized brighness at $36 \mathrm{MeV}$ is micropulse current. The estimated brightness from our previous accelerator with thermionic injector and 20-MeV energy is shown for comparison.

tion and operating parameters have been performed using the FELEX code. ${ }^{x}$ This small-signal gain predicted by FELEX is approximately $200 \%$. Our experimental measurements show gains in excess of $160 \%$. The start up of the optical macropulse is shown in Fig. 1.8. We have found the small-signal gain measurements difficult to perform. FELEX simulations show that the gain is large for only a small number of micropulses (approximately 10 micropulses or $0.5 \mu \mathrm{s}$ ) at the start of the macropulse. Furthermore, if transients at the start of the macropulse can make the initial lasing unstable.
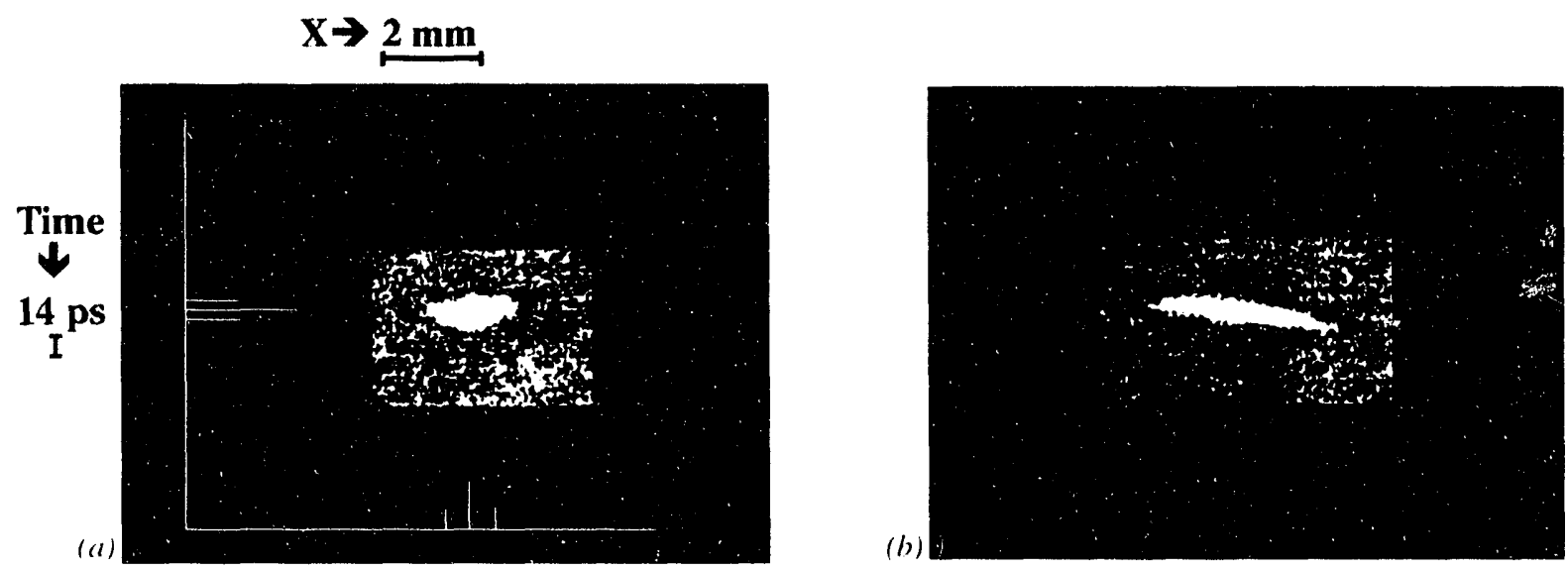

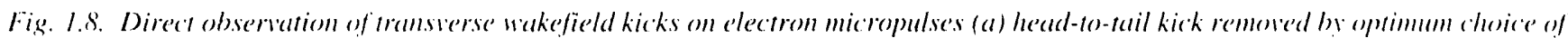

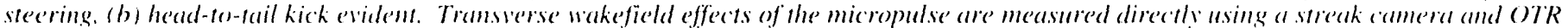

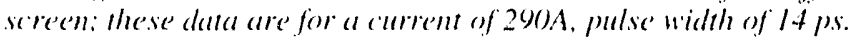


When the FEL is lasing at wavelengths near $3 \mu \mathrm{m}$, very strong sidebands are evident (Fig. 1.9). Such strong sidebands would reduce the efficiency of a high-extraction FEL with a tapered wiggler. Recently, we directed our efforts toward the study of sideband suppression techniques. The first suppression technique we attempted was the introduction of Brewster plates into the resonator cavity. ${ }^{5}$ We found that these plates strongly suppress the sidebands. The suppression occurs at all plate angles including Brewster's angle. At Brewster's angle the insertion loss of the plates is approximately $(0.5 \%$ per pass. We believe that the suppression occurs because the Brewster plates act as a dispersive element. The first sideband is displaced by approximately $3 \%$ in wavelength from the fundamental. Dispersion in the plates results in a round trip path-length change of $17 \mu \mathrm{m}$ relative to the fundamental. Previously we showed that detuning a cavity without Brewster plates by approximately $10 \mu \mathrm{m}$ was sufficient to suppress sidebands. However, detuning the cavity also results in a reduction in the gain on the fundamental. The Brewster plates allow the detuning of the cavity for the sidebands while maintaining cavity synchronism on the fundamental.

We have begun collaborating with Mission Research Corporation to test a new method of sideband suppression. This technique involves using a phase- step mirror as a replacement for one of our regular resonator mirrors." ${ }^{1010} \mathrm{We}$ believe the phase-step mirror technique will be more applicable to high-average power FEL applications than the Brewster plate technique.

\section{Short-Wavelength Lasing}

During FY 1992, approximately 2.5\% of APEX effort was devoted to a DOEfunded program to develop ultraviolet FELs for photolithography of semiconductor computer chips. The goal of the proof-of-principle experiment at APEX ${ }^{11.12}$ was to show the modestly sized FEL could be used to produce ultraviolet light $(250 \mathrm{~nm})$. As part of this effort, we have begun harmonic lasing with both our existing permanent-magnet wigglers and a new electromagnet microwiggler. By the end of FY 1992, we had lased at wavelengths down to $827 \mathrm{~nm}$ with a permanent-magnet wiggler. Using the microwiggler, we observed spontaneous light emission at wavelengths close to $500 \mathrm{~nm}$.

\section{Automation}

The APEX control room was originally designed and constructed in the early 1980s. All controls were manual with no computer intervention. In 1992 we received funds from USASDC for the purpose of developing computer controls and automation for subsystems of APEX. As a basis for our control system, we chose the Experimental

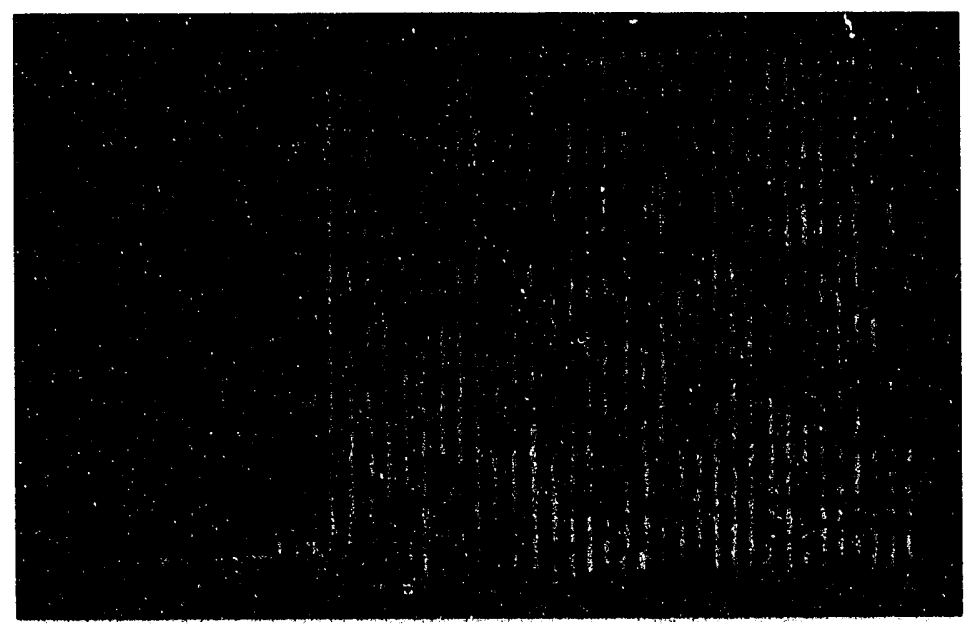

Fig. 1.9. Start up of lasing at $3 \mu \mathrm{m}$, showing a small-signal gain of I60\% per pass. The individual micropulse's are to nis apart. 


\section{References}

1. B. E. Carlsten, J. C. Goldstein, E. J. Pitcher, and M. J. Schmitt, "Simulations of APEX Accelerator Performance in the New Non-Thermalized Photoinjector Regime," (to be published in Nucl. Instru. \& Methods).

2. B. E. Carlsten, L. M. Young, M. E. Jones, L. E. Thode, A. H. Lumpkin, D. W. Feldman, R. B. Feldman, B. Blind, M. J. Browman, and P. G. O'Shea, "Design and Analysis of Experimental Performance of the Los Alamos HIBAF Facility Accelerator using the INEX Computer Model," IEEE J. Quantum Electron. 27, 2580 (1991).

3. P. G. O'Shea, "The Los Alamos High-Brightness Photoinjector," in HighBrightness Beams for Advanced Accelerator Applications, (College Park, MD, June 6-7, 1991), AIP Conference no. 253, p. 182.

4. P. G. O'Shea, S. C. Bender, D. A. Byrd, B. E. Carlsten, J. W. Early, D.W. Feldman, R. B. Feldman, W. J. D. Johnson, A. H. Lumpkin, M. J. Schmitt, R. W. Springer, W. E. Stein, and T. J. Zaugg, "Initial Results from the Los Alamos Photoinjector-driven Free-Electron Laser," Nucl. Instru. \& Methods A318, 52 (1992).

5. P. G. O’Shea, S. C. Bender, B. E. Carlsten, J. W. Early, D. W. Feldman, R. B. Feldman, J. C. Goldstein, K. F. McKenna, R. Martineau, E. J. Pitcher, M. J. Schmitt, W. E. Stein, M. D. Wilke, and T. J. Zaugg, "Performance of the APEX FEL at Los Alamos National Laboratory," (to be published in Nucl. Instru. \& Methods).

6. J. W. Early, J. Barton, R. Wenzel, D. Remelius, and G. Busch, "The Los Alamos FEL Photoinjector Drive Laser," IEEE J. Quantum Electron. 27, 2645 (1991).

7. B. E. Carlsten, J. C. Goldstein, P. G. O'Shea, and E. J. Pitcher, "Measuring Emittance on Non-Thermalized Electron Beams from Photoinjectors," (to be published in Nucl. Instru. \& Methods).

8. B. D. McVey, "Three-dimensional simulations of FEL Physics," Nucl. Instru. \& Methods A250, 449 (1986).

9. J. E. Sollid, D. W. Feldman and R. W. Warren, "Sideband Supression for FELs," Nucl. Instru. \& Methods A285, 153 (1989).

10. A. H. Paxton and M. J. Schmitt, "Sideband instability in FELs - a new technique for supression," IEEE J. of Quantum Electron. 26, 1167 (1990).

11. B. E. Newman, R. W. Warren, J. C. Goldstein, B. E. Carlsten, M. J. Schmitt, S. C. Bender, D. W. Feldman, and P. G. O'Shea, "The Los Alamos POP Project: Design of FEL Experiments in the Ultraviolet and Beyond," Nucl. Instru. \& Methods A318, 197 (1992).

12. R. W. Warren, P. G. O'Shea, S. C. Bender, B. E. Carlsten, et al., "Lasing in the Ultra-Violet with a Microwiggler," (to be published in Nucl. Instru. \& Methods). 


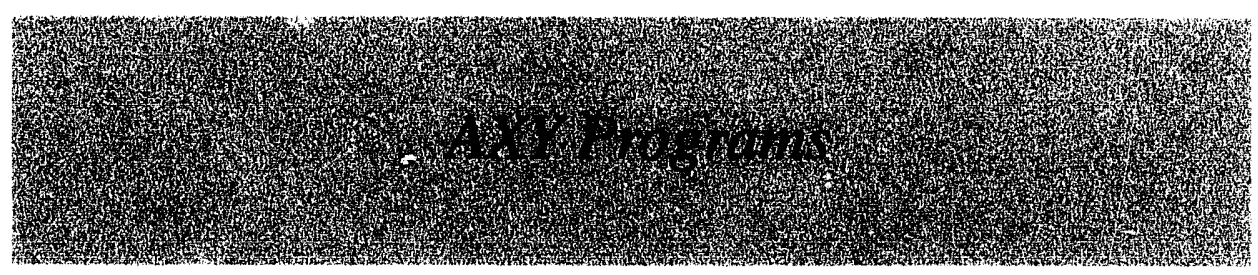

\section{Status Report for AXY Programs}

Potemtial applications of high-power accelerator-driven spallation neutron sourese expanded dramatically during 1902. The Department of Energy (D)E) funded an 18-month design study of Accelerator Production of Tritium (APT), the National Academy of Siciences reviewed Accelerator Transmutation of Waste (ATW), and a Laboralory-Industry collaboration put forward a proposal to study Accelerator Bancel (onversion ( $\triangle B C$ ) of Russian weapons plutonium. A los Alamos proposal was also submitled to the Defense Nuclear Agency (DNA) for building the first stage of a high-power comtinuous-wate (cw) proton linale fromt end to demomstratte key aspects of the echmology. The steadily increasing number of programs are now referred (1) collectively ats $A X Y$. where the $A$ stands for high-power accelerator, and the remaining letlers represent the specilic application.

Comsiderable progeress was made in developing atcelerater conceptual kesigms. in identifying and addressing hey technical isstes for high-power ch linacs. and in examining important design questioms, such als mechanisms for the generation of heam halos. A 1 week workshop on high-power accelerators and spallation tallgets was held in Norember helueen los Alamos designers and colleagues form ITl:P and MRTI in Moscous and from onher instituter in Russia.

\section{AP'T Program}

following the stecesstul oulcome of a JASON pand review of lon Alamos and Browhlaren propesals for Acectcrater Production of Tritium in Janualsy. the Dot: decided to lund an I8-month study of the concept. The study was structured as a collaboration between Los Alamos National Laboratory (LANL, Brookhaven National Laboratory (BNL), Sandia National Laboralory (SNL.), and was initially managed hy the New Production (NP) office of DOE. NP's purpose is to provide APT input to the Programmatic Environmental Impact Statement (PEIS) for Complex 21 and to assess the lechnical feasibility and costs of the AP'T concept. LANL is responsible for the APT accelerator design and for a larget/ blanket and processing system batsed on converting the tritium decaly product 'He back into tritium. BNI is responsible for a target/blanket system similar to the one presented to the Energy Rescarch Advisory Board (ERAB) in 1989, based on a matrix of lead and LiAl rods. SNI. is responsible for the envirommental, satiely, and health evaluation of the APT design and specifically for coordinating the PEIS imput. The APT study is lleshed out by contracts with industry for enginecrimg support. costing, and A/E services lo define the Balance of Plant (BOP).

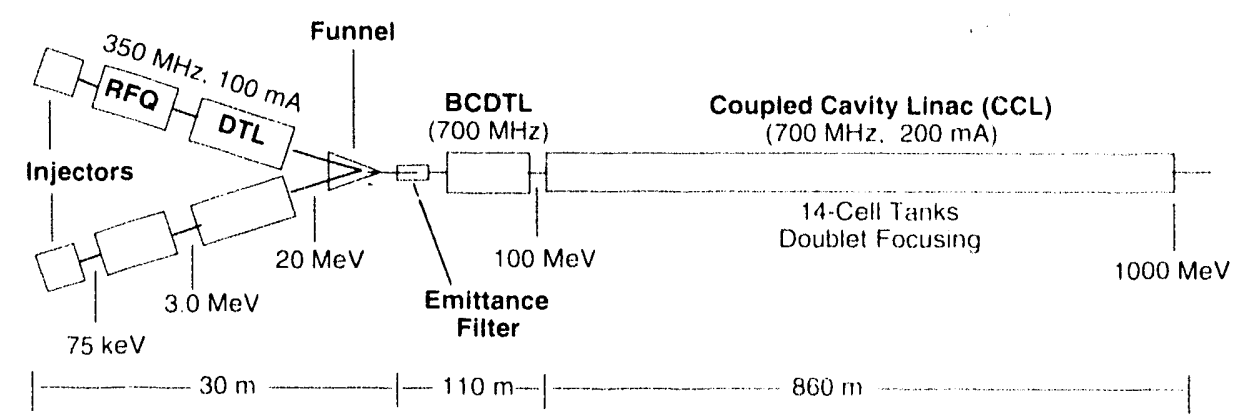

With the closure of the NP affice of DOF: at the end of 1992, the APl program is a be transferred to the Delense Programs (D)P aftice.

\section{Accelerator Design}

The APT linate reference design catls for a 1-cicV, 200-mA cw protom linac. The high-energy-beam transport serves one of iwo allemate production target blankel assemblies, with a specification of $75 \%$ overall plant analability. The linac architecture pictured in Fig. 1.10 consists of a fumneled frome end that combines 10()$-m A$ beams from two 20 MeV 3.5()-MHz linacs, followed by (wo 70)()-MH/ high-energy accelerating structures that provide a I-(ieV. 2()()mA ouput. lach of the low energy linaces consists of a 7.5-keV proton injector, a 3-MeV radiofiequency quatdrupole (RFQ), and a conventional drift-lube linac (DTL). The acederating structure following the funnel is a short-tank bridge-coupled drift tube linac (BCDTh, ) that comtains no yuatdrupole magnets in the drift lubes. The final accelerating structure, which is about 8.5()$-m$ long, is a coupled-cavity linat (CCl.) in which cach 14-cell lank is driven by a single high-power klystron. A key feature of the high-energy struclures is a large aperture-to-beamsise ratio in order lo meet the very low beam loss requirements needed lior

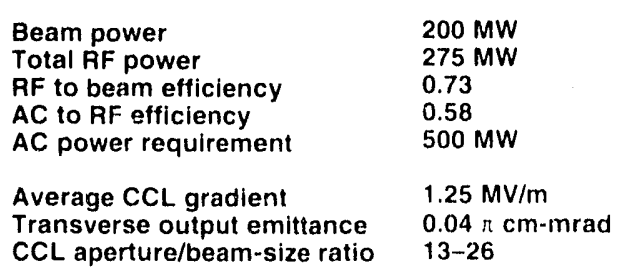

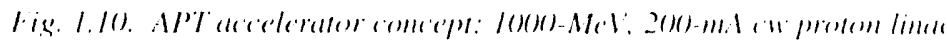


ensuring hands-on maintenance. Short tanks and a quadrupole doublet focusing scheme help in the attainment of this objective. A low accelerating gradient $(1.25 \mathrm{MV} / \mathrm{m}$. average $)$ in the CCL provides high of efficiency and minimizes life-cycle costs.

A high-energy beam transport (HEBT) system conveys the protons from the end of the linac to one of two alternate tritium production target/blanket assemblies. While one of these assemblies is in production, the other can be serviced. The transport system consists of a doublet focusing lattice matched to that of the linac. followed by an achromatic bend, and terminating in an expander based on nonlinear optical elements. The expander converts the small-size nearly Gaussian beam distribution from the accelerator into a large area uniform density distribution at the target face.

\section{Program Events}

The APT program received a status review in September 1992 of the reference design. Key PEIS input data, such as estimated radioactive air releases, are to be provided to Sandia by the end of January 1993. A comprehensive review of the complete APT system design, including PEIS impacts, is scheduled for early March 1993. A set of topical reports on the design of all aspects of APT, with participation by industrial partners. will constitute the final FY 199.3 program deliverable.

\section{ATW Program}

The Laboratory is continuing to study Accelerator Transmulation of Waste (ATW). The initial application of this scheme was focused on destruction of the accumulated high-level radioactive wastes at the DOE's defense production sites. principally al Hanford, but this vear the study emphasis has shilted (o) include transmutation of spent commercial power reactor fuel. The base line ATW system being considered incorporates a 160()$-\mathrm{MeV}, 250-\mathrm{mAcw}$ proton linac that drives four high-lher- mal-flux neutron sources surrounded by $\mathrm{D}_{2} \mathrm{O}$ blankets in which the materials to be transmuted flow in aqueous-based carrier loops. This system could transmute the actinide and fission product waste of about eight 1-GWe light water reactors, converting it to stable or shorter-lived products that do not require deep geologic storage. A more advanced high-temperature ATW system, based on a helium-cooled graphite blanket with molten salt carrier loops, is also being examined. It promises higher electrical efficiencies, and opens up the possibility of accelerator driven subcritical fission systems that could convert fertile material to fissile fuel, burn the fuel to produce energy, and transmute all long-lived nuclear waste generated in the process.

\section{DOE Environmental Restoration and} Waste Management (EM) funds have supported chemistry and material balance studies for ATW, while accelerator and target/blanket design have been supported by LANL-directed research and development (LDRD) dollars. Key activities this year have revolved around a comprehensive review of separations technology and transmutation systems (STATS) for high-level nuclear waste. This review is being conducted for the DOE by a special panel of the National Academy of Sciences. The ATW accelerator design was reviewed by the STATS panel transmutation subpanel in April as part of a complete review of the Los Alamos ATW schemes.

\section{ABC Proposal}

In FY 1992 an industry-led consortium. with Los Alamos as a major partner. proposed to study a special ATW system dedicated to converting returned Russian weapons plutonium. The proposal, led by Grumman Aerospace Corporation (GAC), is aimed at funds made available by the 1992 NumnLugar legislation dealing with US assistance to Russia in dismantling nuclear weapons that have been eliminated in recent stockpile reduction agreements. The proposal is for a study that would develop a reference design for an Accelerator Based Conversion $(A B C)$ system that would be built in Russia as a joint US/Russian technical project. The study would also assess the feasibility of implementing an Integrated Test Facility (ITF) for prototyping the $A B C$ process at an upgraded version of LAMPF. Several interactions between LANL, US industrial participants, and relevant Russian scientific institutes and government agencies took place throughout the year, culminating in a Moscow workshop on target design and chemistry for ATW/ABC systems and a Los Alamos workshop on ATW/ABC accelerator design. Both workshops were held in November 1992.

\section{DNA Proposal}

Los Alamos also submitted a proposal for funding to the DNA for developing a high-power $\mathrm{cw}$ accelerator front end that is generic to ATW, ABC, and APT applications and that also has application to a future LAMPF front end replacement. Accelerating structure frequencies have been chosen to match those of LAMPF. Thus, if the initial program can be extended into a 4- to 5year program, it will be possible to develop a complete demonstration of the front-end $\mathrm{cw}$ accelerator technology for transmutation systems. The hardware would then be converted into the first stage of a LAMPF accelerator upgrade suitable both for driving a recently proposed advanced spallation neutron research facility (LANSCE II) as well as an ITF that could evaluate ATW technologies. The proposal to DNA includes an assessment to determine what is necessary to upgrade the LAMPF linac's power level for ATW technology and to implement the new technology in demonstration facilities. The proposal also includes a study of the intense pulse provided by the Proton Storage Ring (PSR) for weapons effects measurements. 


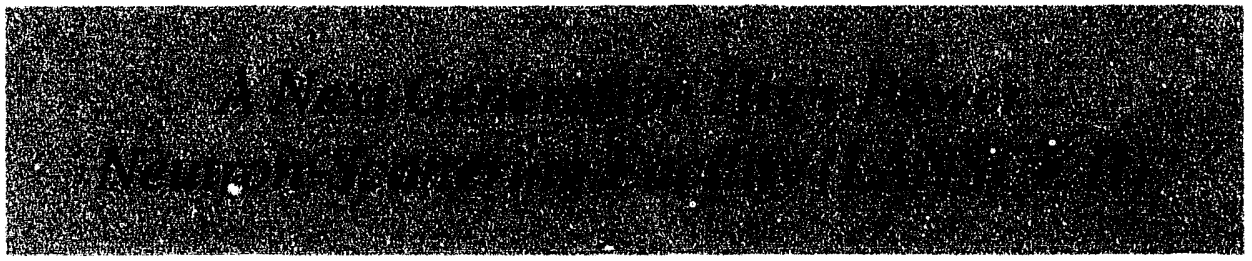

A new initiative is under study by a Los AI ...os National Laboratory (LANL) interdivisional team, with strong AT participation. This large project would greatly increase the capabilities of US neutron scaltering facilities, including the Los Alamos Neutron Scattering Center (LANSCE). LANSCE now provides the world 's most intense burst of neutrons for research in a wide range of condensed-matter topics and biological studies. A nominally I-ms $H$ macropulse from the Clinton $P$. Anderson Meson Physics Facility (1.AMPF) containing some $3 \times 10^{1.3}$ particles is accumulated for about 2500 turns in the Proton Storage Ring (PSR) and is then delivered to a refiactory metal target in a $2(0)$-ns burst to produce the desired spallation neutron pulse. The net beam power to the target approaches $80 \mathrm{~kW}$. A rival source. ISIS. operates at the Rutherford Laboratory in the United Kingdom, and other smaller spallation sources throughout the world accommodate a large community of neutron scattering workers. Such pulsed-spallation sources provide a much higher peak neutron intensity though less average intensity) than do nuclear reactors designed for neutron research. Hence, although reactors are useful to neutron scattering work, they lack the pulsed character essemtial to much of the rescarch.

Recent interest hats arisen regarding sources of substantially greater neutron intensity than that of LANSCE. The European community has undertaken a 2-ycar study for a 5-MW spallation source that would exceed existing reactor atverage intensity. Discussions within I os Alamos this spring provided impetus for a similar proposal from the lathoratory. In particular, funding for I.AMPF as a nuclear physics facility is scheduled 10 end with F:Y 199.3.
Hence, the conversion of LAMPF to a high-power neutron-source driver is very attractive, both in preserving the present facility and in meeting mational research goals. Additionally, reusable parts of the linac and the extensive infrastructure built up over the past 25 years would substantially lower the construction cost and effort of such a spallation source over a "green-ficld" facility. The notion of emphasis on neutrons at Los Alamos is not new; several years ago AT personnel, in a study known as LANTERN, suggested that the future of the mesa was in neutron production and advocated this direction for several purposes, including waste transmutation, defense applications, and energy production as well as neutron scattering. Most of the recommendations of that study are now under active pursuit by the Laboratory.

In August 1992, a site visit by the Department of Energy`s Basic Energy Sciences Advisory Committee (BESAC) provided us with the opportunity to present our proposial. A conceptual design for a 5-MW facility had been proposed internally and was quickly augmented with technical detail as well as a preliminary cost analysis. Presentations were made by AT, LANSCE, and Medium Energy Physics (MP) personnel to the commiltee. Shortly thereafter, a Los Alamos contingent was invited to a workshop in Chicago, held in conjunction with the BESAC committec site visit to Argonne National Laboratory, 10 present our proposal to the BESAC accelerator subcommittee. Again, a crash effort was mounted that led to a more detailed design and cost assessment. The presentation went smoothly and appeared to be well received, thanks to a great deal of effort by mumerous individuals in the Division.'
The proposal noted that LAMPF is currently a I-MW facility with a very reliable coupled-cavity linac (CCL) section that comprises over $90 \%$ of the linac. The proposal includes replacing the present $200-\mathrm{MH} z$ front end with a 40()$-\mathrm{MHz}$ radio-frequency quadrupole (RFQ) drift tube linac (DTL) combination, both to increase reliability and (o) provide an upgrade path to even higher power. The beam (all 20 MeV) then enters a new $8(0)-\mathrm{MH} z$ DTL for acceleration to $100 \mathrm{MeV}$ and matching to the present CCL. This arrangement enables funneling at the 20-MeV level for an upgrade to $5 \mathrm{MW}$ of beam power as shown in Fig 1.11. No upgrade of the CCL rf power is required in the 1-MW case because the linac presently carries current at this level (at 12() $\mathrm{Hz}$ ) and hence provides the same average power. Additionally, by doubling the front-end frequency, the charge per bunch is actually reduced from the present LAMPF operating conditions.

We designed an accumulator ring with achromatic bends and a 14()-m-circumference "race-track" configuration for the 1-MW case. The achromatic bends promote a high degree of linearity that is undisturbed by chromaticity corrections. Injection is accomplished by single-stage foil stripping and phase-space painting is planned. There are many issues in the required accumulation of over 10$)^{1.4}$ particles in the ring (several times that successfully accumulated in the PSR). We will draw heavily on the lessons learned in the PSR studies to increase storage capability. Beam losses and the electron-proton instability are particular issues encountered in the PSR to which we must pay close attention. Becaluse of the increased ring size, the resistive-wall instability, unimportant in the PSR, will dominate and active damping will be required. A sophisticated rf system using several harmonics will also be required to keep the beam longitudinally confined to provide an extraction gap. When we include the sophisticated hump system for injection painting and an extraction kicker, it is clear that the ring requires extensive pulsed-power implementation. 


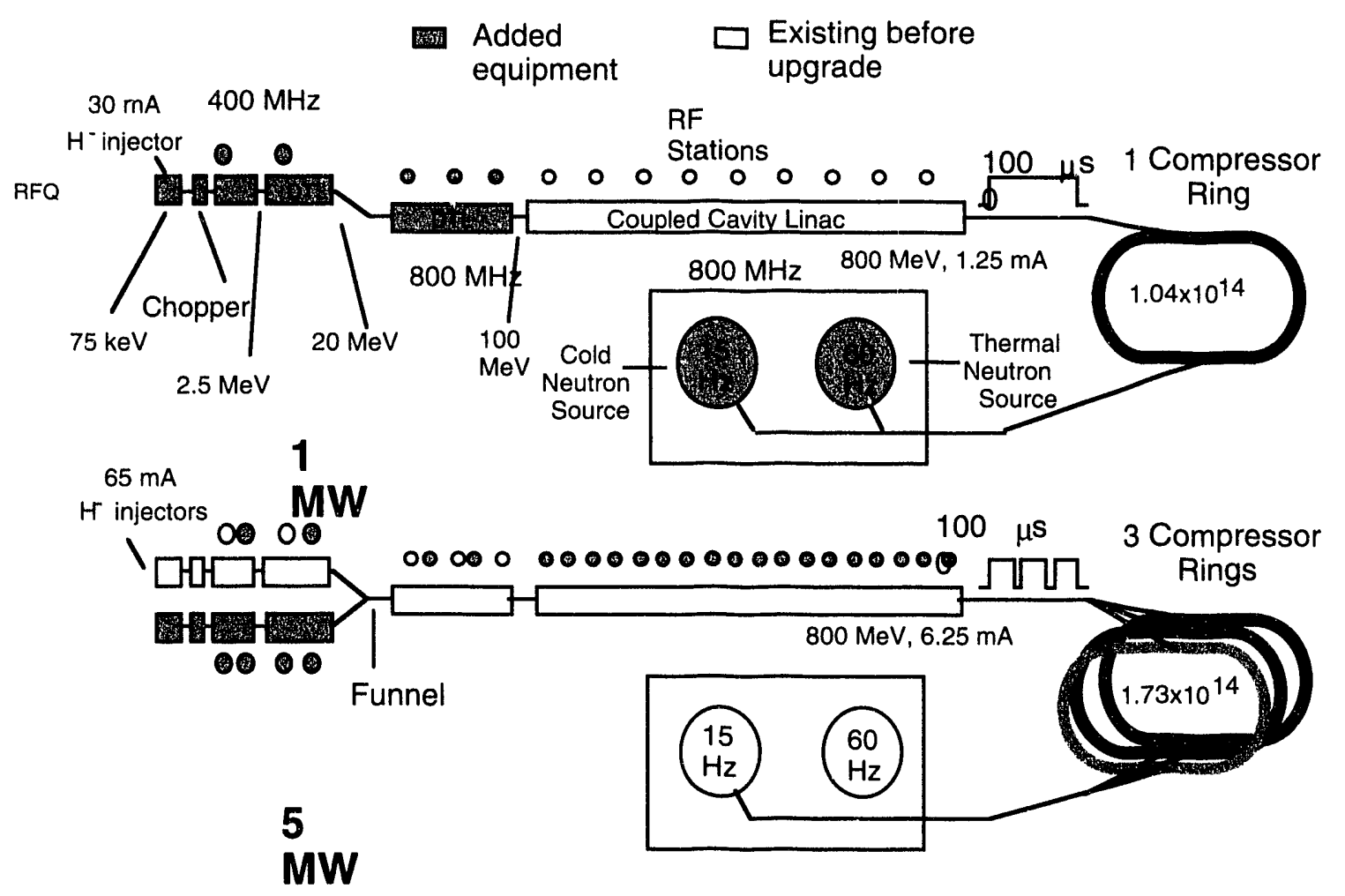

Fig 1.11. Upgrade path for LANSCE II. The I-MW case is shown at the top. In a 5-MW scenario, a funnel at the 800-MHz DTL is completed and the rf power throughout is doubled.

After one-turn extraction from the ring, the highly compressed beam pulse is conducted to two target stations, where neutrons are produced by beam impingement on a refractory metal target. The neutrons interact with energy-moderating materials and then pass through holes in the shielding surrounding the target for use in a wide variety of instruments. The LANSCE staff will now study the high-intensity target design and develop plans for a research facility.

The upgrade to $5 \mathrm{MW}$ would require doubling the rf power throughout the linac and increasing ion-source intensity to $100 \mathrm{~mA}$ as well as completing the funnel at $20 \mathrm{MeV}$. Two additional rings would be required for sequential accumulation; storage in each would be increased by almost $70 \%$. The parameters of this upgrade are very stressing and we must prove that they are attainable. The major issues identified so far include
- chopping the beam on a l-ms time scale to leave a gap for extraction in the ring,

- current carrying ability of the $C C L$,

- ring stability and storage time,

- system reliability, and

- system particle losses and activation.

Some of these issues lead us to conclude that a higher energy (up to 2

$\mathrm{GeV}$ ) would be helpful because the current needed to produce the required power is decreased and ring stability is enhanced. Such an option would require either an afterburner linac or replacement of the CCL. In either case, a superconducting linac provides an attractive option.

We expect to have a preliminary design proposal ready for August 1993, which would position us for possible construction funding in 1996. Our initial estimates for the 1-MW-scenario construction cost are over $\$ 500$ million; the planned upgrade would cost an additional $\$ 340$ million.

\section{References}

1. A. Jason, R. Hardekopf,

G. Lawrence, R. Macek, R. Pynn, and G. Russell, "LANSCE - The Los Alamos '1-MW' Spallation Source," Spallation Source Workshop, Chicago, IL, September 1992, Los Alamos National Laboratory document LA-UR-92-3497. 


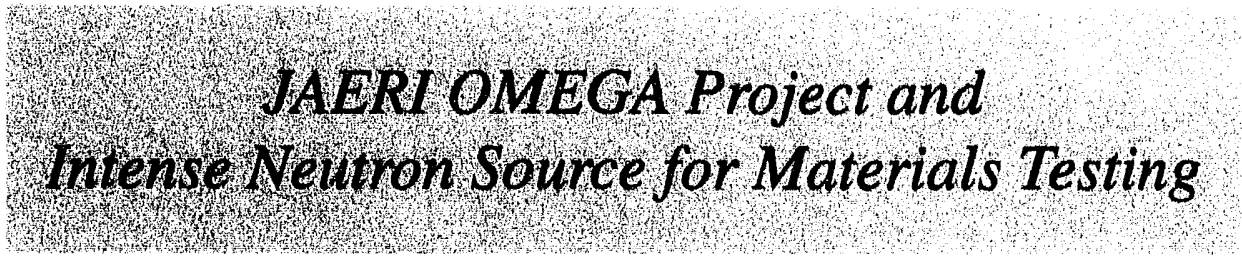

\section{JAERI OMEGA Project}

The Japan Atomic Energy Resealleh Instilute (JAIERI) continters its ()ML:CA Program activities in the evaluattion of new options lor disposing of radionctive waske, and again provided funds to AT-Pivision lo explore system layout and optimizantion for the lo(0) MeV. 10)-30 ma cow linate recpuired and at) pursue studies of heam loss controls.

This years sepere included al collection of memos on $\Lambda \mathrm{T}$-Division point-design activities for atcederaturs of this class as they would be applied to ratdwaste transmutation or tritium production. The Al:C'L Resciarch Company Was subtasked lo contribule their ideas on the ion source and low-betat atecelerallor components.' The report continuess will a discussion from $\mathrm{AT}-4$ on manuficturing process and cos models for a high-beta coupled-calvity linate (c) (C) and its subsylems, in which thermal frequency shifs, vatumm. and electromagnel yuadrupole considerations are highlighted. The two concluding chapters ance new work on the space-charge physics ardhitecture of

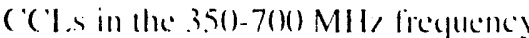
range and scaling and optimizaltion studies of these long linaces. The point designs were crablualed in termon of letal be:mo sisc along the alcecterator, and prediminary criteria were deselened for making some of the many choices reyuired for optimizatlon.

\section{Intense Neutron Sources for Materials 'Testing}

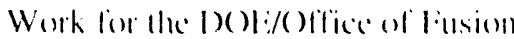

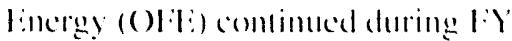
l() 2 as lle mallerials commmunily laid the grommelwoth for making an internat liomal proposal. Mectimgs al the inlemantional community realfirmed the cindidacy ol the D)-1.i (deulcorm hombarding a lithium targed) as (he neatr-kem kedhology of chosice. The IS colfort was organised under an andhoce commintec chatred hy ()ak Ridgec Nallomal l.aboralory (o) () NI.). lo hegin preparing matterial for making and intcrnational proposil alrombl the end of fiY9.3. Fonclioms and reyuirements

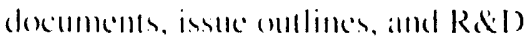
reguirements decoments were prepaned for all Il: Mall: scalle lakeility, requirimg at $35-40$ MeV (cw deulerom limac al 110 lo 2.5() $\mathrm{m} / \mathrm{A}$ (N) current.

In parallel, lla IAl:RI lincrey Selective

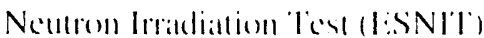
facility proposal colfort contimes. This faccility woukt use a $5(1)-10() \mathrm{mm}$ denteren linate for lusion and hasic

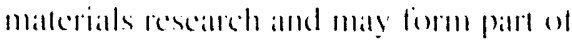
a staged intermational colfort. JAI:RI provided lunds lo Al bovision lon comceptual design studies directed mo

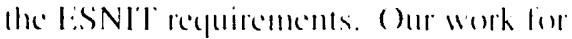
the linst report included design of a bealln redistribution system thall wases

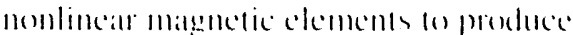
a reclameulat beam spol on targed that is neanly unitorm within the rectangle and derom cleamly to dery low levels (m)side. Inilial mork on (n)

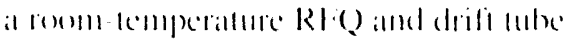
linatc was completed, and decorelicall develupment included combideration of a mormall form allaly is of ale amharmonic ascillator molel and a

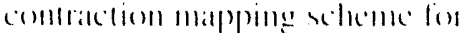

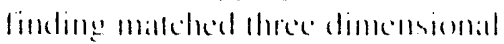
rarclupes
A major new comsideration in alecel

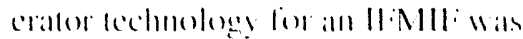
proposed follec commomity by the

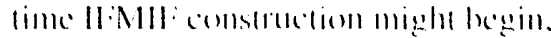

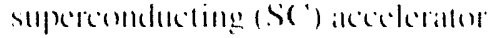

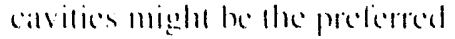

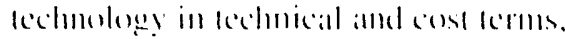

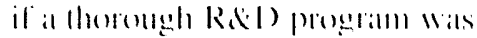
supported before final commomiment to

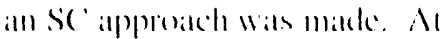

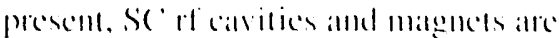
beinge usced in manly Hew accolertallor installattons and alle he heimning lo prove dheir reliabilies and eos ellediveness. The very high-intensily mathines moker considerantom in $\mathrm{A}^{\prime}$ 1)ivision and anndher dimsemsion: becallse high-intensily ion heallos halve

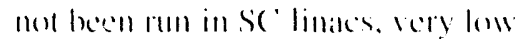
beallt fors is a key issme, and the machines mous roun very rediably in al firelory cowionment will mam operatling hours pere your. ('omsider

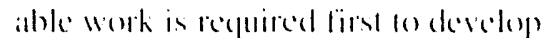

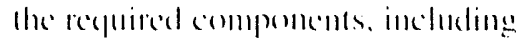
calvilies, il pewere complers, imstrumen-

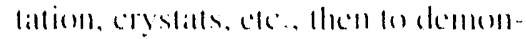
statc the compunems with a high

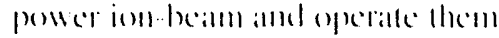
reliahly lior some pertiol. The philosophy and procedures lon maintemance

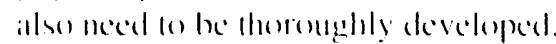
Homerer. Hore are gend lechnical

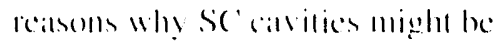

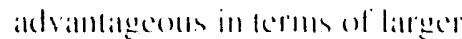

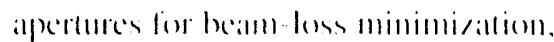
and higher anceleranting gradicons lon shorter lengelh. In comblomallon wilh pussible salvimes in caprilal and copectially operatlin!? cosks, we hatre

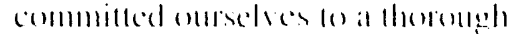
coaluation of the Sc pussibilits

\section{References}

1. (i. 1: Ma Michatel B. (i. C'midley

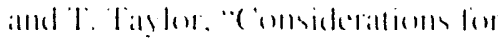

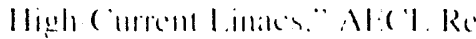

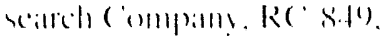
(April (2)? 


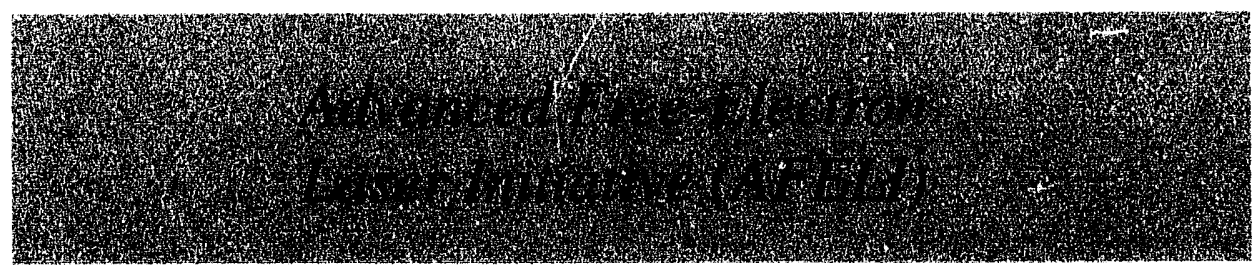

\section{Introduction}

The Advanced Free-Electron Laser Initiative (AFELI) is funded to support high-quality basic and applied research in the field of Advanced Free-Electron Lasers (AFELs) and to demonstrate these advanced technologies. AFEL research will provide an understanding of the performance and engineering limits of free-electron laser (FEL) systems, thereby strengthening the science and technology base for existing and future Laboratory and national FEL initiatives. The goal is to build a second-generation FEL system to research and develop advanced components. This system will incorporate state-of-the-art components and be friendly and robust. Research and developmental areas include the various subsystems of the FEL: the electron source, accelerator, wiggler magnet array, optical system, diagnostics, and control system. State-of-theart components include ultrabright, high-gradient, low-loss accelerators: electromagnetic microwigglers; emittance-preserving magnetic bunchers: and advanced optical resonators. This second generation of FELs, referred to as compact FELs because of their size, has many potential applications in industry, medicine, and research.

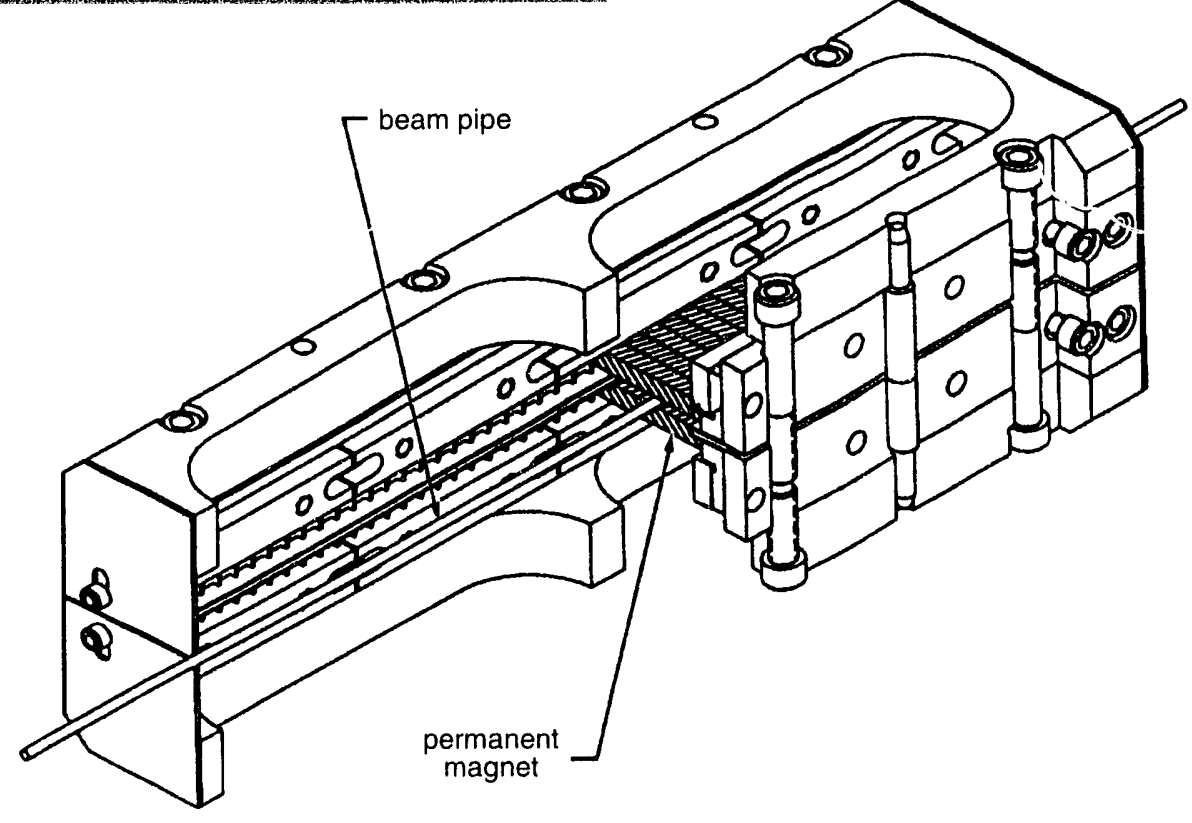

Fig. 1.12. Permanent magnet wiggler.

\section{Description}

The present design of the compact FEL consists of a high-brightness accelerator and a microwiggler. The electron source is a laser-driven photoemitter that produces $2-\mathrm{nC}$ and 10 -ps-long micropulses at a rate of $108 \mathrm{MHz}$. The macropulse is $20 \mu \mathrm{s}$ long and has a rate of $10 \mathrm{~Hz}$. The electron beam is accelerated to $20 \mathrm{MeV}$ by a $1.2 \mathrm{~m}$ on-axis coupled structure operating at 1300 $\mathrm{MHz}$ with a field gradient of $22 \mathrm{MV} / \mathrm{m}$. The accelerated beam has excellent beam quality with a transverse emittance of less than $3-\mathrm{mm}-\mathrm{mrad}$ and an energy spread of $0.3 \%$.

Two types of wigglers will be used: a permanent magnet wiggler and a pulsed-current wiggler. The permanent magnet wiggler (Fig. 1.12) has $241-\mathrm{cm}$ periods. The peak magnetic field is 0.4T on-axis. The pulsed-current microwiggler (Fig. 1.13) is $10-\mathrm{cm}$ long with a $3-\mathrm{mm}$ period and has a slotted tube design. It is driven by a pulsed dc current of $10 \mathrm{kA}$ to generate a magnetic field up to $5 T$ on-axis. The length of the optical resonator is $1.4 \mathrm{~m}$. Including operation at higher harmonics, the laser wavelength extends from $7 \mu \mathrm{m}$ down to $0.4 \mu \mathrm{m}$.

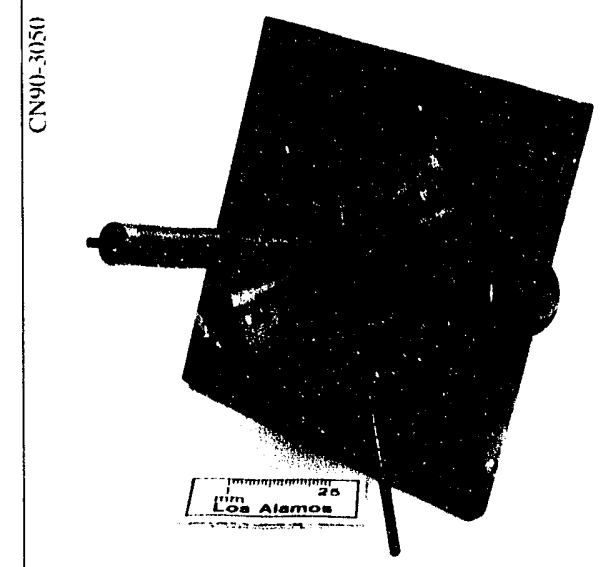

Fig. 1.13. Pulsed-current microwiggler. 
The experiment will use the control system developed by AT-8. This control system will be used initially tor monitoring system parameters. Lafer in the program, the control system will be used for active control.

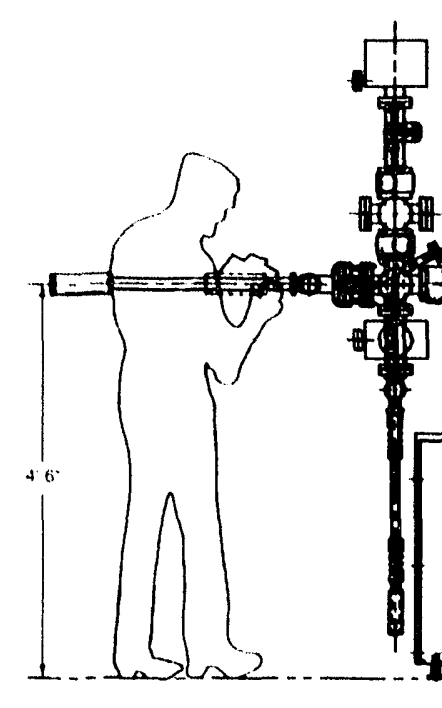

The FEL facility is at Meson Physics Facility, Technical Area 53, building 14. The acederator and FEL. atre so compact that they can he housed in at $1211 \times 25$ fi vault.

The accelerater structure has been fabricalled (Fig. 1.14), and schematic of the AFEL experiment is shown in Fig. 1.15. The beamline, shown in Fig. 1.16, consists of permaneme magnet yuadrupoles and dipoles; permanent magnet elements atre used o improve reliability and compactness. The permanent magnet wiggler has been construcled, and the pulsed lube wiggler is under construction. The first beam was ohtained in Junc of 1992: the first FEL operation is expected in the spring of 1993.

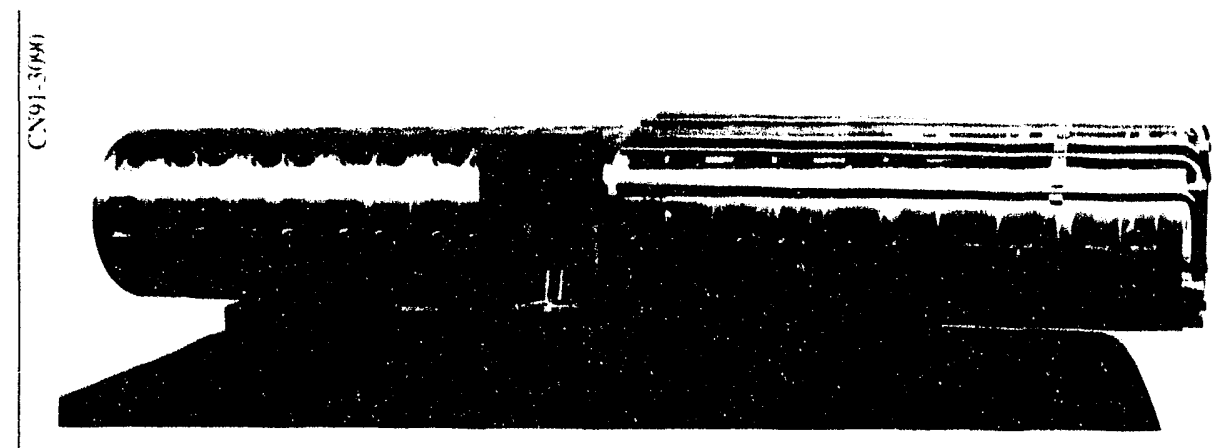

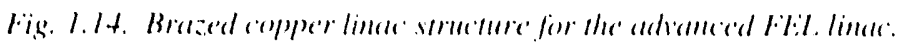




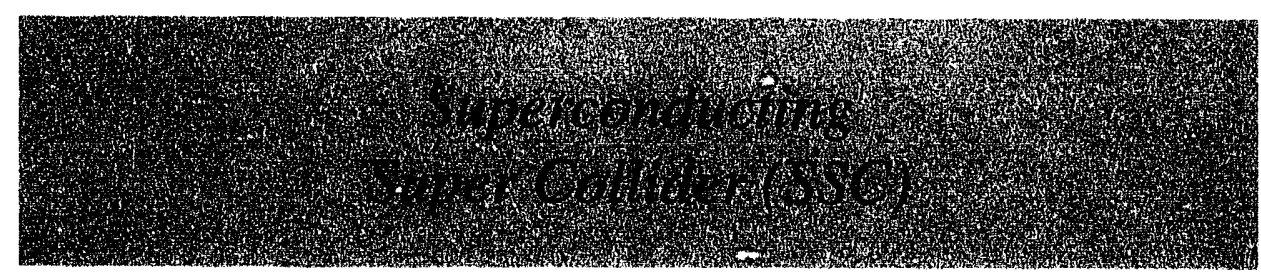

During 1992, several groups in AT Division were involved in providing support to the Superconducting Super Collider (SSC) Laboratory near Dallas. These activities are summarized here and covered in more detail in the specific groups involved.

In the SSC linac area, AT- 1 has designed, fabricated, and delivered a 4 vane, $2.5-\mathrm{Mev}, 428-\mathrm{MHz}$ radiofrequency quadrupole (RFQ) to SSC. AT-5 fabricated a tetrode amplifier for this RFQ, which SSC has coupled to and successfully tested on the RFQ.

AT- 1 has continued to provide support in the cavity design for the $1284-\mathrm{MHz}$ coupled-cavity linac. Additionally, AT1 provides beam-simulation support to the SSC Linac Group on an as-needed basis. AT-3 is modifying the design of some adjustable-gradient permanentmagnet quadrupoles developed for the Ground Test Accelerator (GTA) that will be used in the SSC RFQ-to-drift tube linac (DTL) matching section. These quadrupoles are very compact in comparison to equivalent electromagnet designs. In the low-level if area, AT -5 continues to work closely with the SSC if group to provide a low-level rf control system, using development work done for GTA.
In the low-energy booster (LEB), AT-7 has carried out beam-dynamics calculations to better predict and control the effects of beam loading in the rf cavities. AT -5 collaborated with SSC in the design of the perpendicularbiased ferrite-loaded of cavities that must swing from 47.5 to $60 \mathrm{MHz}$ in 50 msec at a $10-\mathrm{Hz}$ repetition rate. AT-1 is also providing rf-cavity design support. AT- 3 and AT- 5 provided modeling support in the design of the $10-\mathrm{Hz}$ resonant-circuit ring magnet system. AT-3 is consulting with the Accelerator Diagnostics Department on the diagnostics design. Similar support in all these areas is being provided for the two other booster rings.

In the collider rings, AT-3 provided support in the prediction of the transmission-line modes in the long strings of superconducting magnets. Because the superconducting magnets form a nearly lossless transmission line, power supply ripple can propagate long distances with little attenuation and lead to emittance growth. AT-3 has proposed a way to damp these traveling waves and limit the magnetic field ripple to less than 1 part in $10^{\prime \prime}$.

In the accelerator controls area, SSC critically evaluated several control systems, both commercial and in use at other national laboratories, and selected the AT-8-designed Experimental Physics and Industrial Control System (EPICS) to be the baseline control system for use on all accelerator systems at SSC. EPICS is also being used in the GEM detector collaboration. 


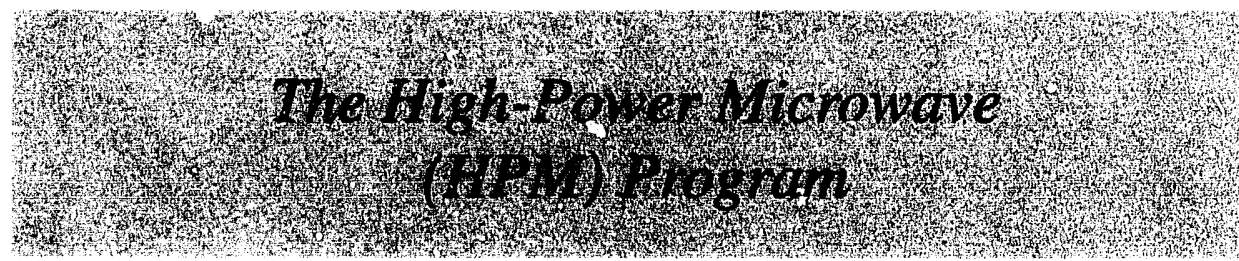

AT Division maintains a program management and program development effort in high-power microwaves (HPM) on behalf of all organizations at the Laboratory with expertise applicable to that lechnology: Laboratorywide program manigement of HPM is resident in AT Division because of the substantial concentration of the expertise in the division. Other divisions currently involved are $A$. CLS. IT. M. MEE. MST. $P$ and $X$. This section presents an overview of that effert. which is described in detail under $A T-9$ atetivity since virtually all division work in this area is conducted in that group.

It has, been recognied from the earliest daty of radis that ele tromagnetic radiation (EMR at: it watelengtios hats potential an al weapon. Although lechnology did not effectively upport this application of EMR until resently becaluse of limitations on of pe ser at appropriate watelengthe it has now hecome feasible to u.e EITR a disrupt o! destros military electronics at useful ranger. Vulanerahilit! considerations have show $n$ ihat the appropriate watelengtha are in the microw are regime. Variour agencier of :he Department of Defence: Dold D hate attive programo to develop HP.M weapom. The lathorator: and division have participated for at mumber of years. because of the unique capabilities here and heciane of potential $\because$ nergi'm with accelerator applications within the Laborator! minion. an moted helous.
The DoD has long been concerned about the accidental adverse effect of HPM on military systems, such as that aboard modern naval ships where nearby systems can interfere with one another. With the growing possibility of intemional interference or destruc.. tion by a microwave weapon, this concern has multiplied and resulted in extensive programs to understand the vulnerability of US military systems to HPM. A related interest in HPM effects resultcd in funding by the Department of Energy (DOE) and its predecessor agencies as well an the DoD beciase of the importance of the "electromagnetic pulse" from nuclear weapons. Again becaluse of the alvailability of unique of sources. insirumentation, and facilities, the Laboratory and division have for many vears participated in these vulnerability programs.

Future generations of high-performance If accelerators will require amounts of if power well heyond that available through a reasonable number of current technology sources. Thus. a strong source-development effort is an important part of the nation $s \mathrm{rl}^{\circ}$ atcelerator program. Work con advanced sources for this application has ator heen an importan part of the division ' past HPM activity.
The main thrusts of HPM program development have been to participate actively in national HPM program management and to detect and pursue opportunities to apply the division's unique capabilities to HPM problems of national importance. HPM program management is intended to ensure effective technical and financial execution of programs, once funded. and to conduct sponsor liaison. To learn about the technical work performed in this areat, the reader is invited to review the section of this report that covers Group AT-9. 


\section{Neutral Particle Beam (NPB) Power Systems Highlights}

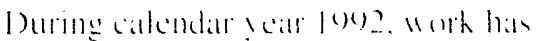

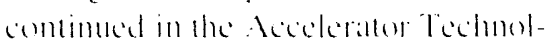

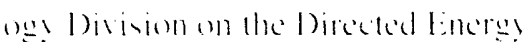
He:apon Powcr Integration

(1)

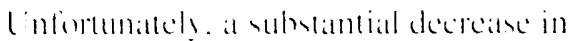
program fumeline callecel a redertion in the number of cedmologers that could he puraced.

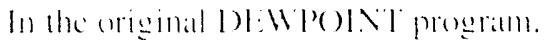
there wat a dual path for all critical

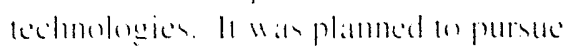

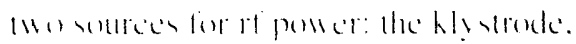

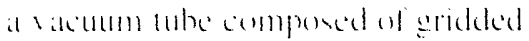

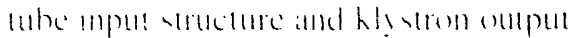
strle!ture. and a solid-slalle amplitice.

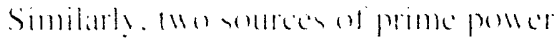

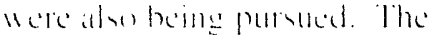

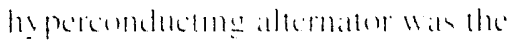

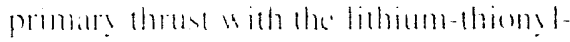

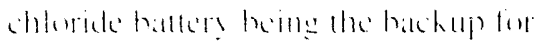

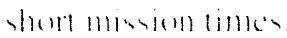

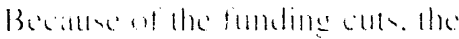

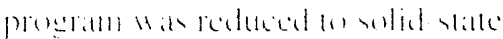

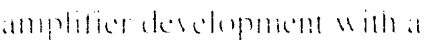

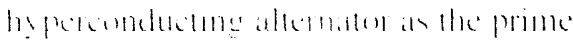

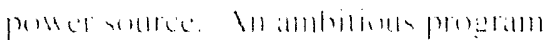

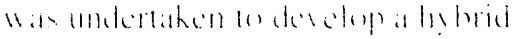

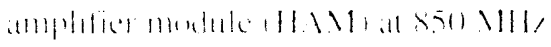

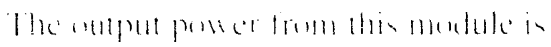
de ienced whe I h th and the moluke

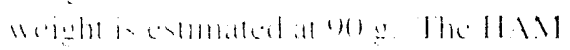

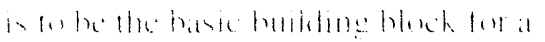

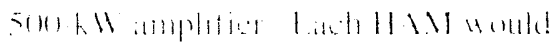

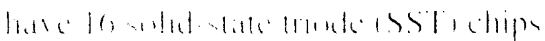

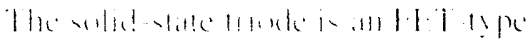

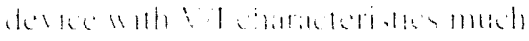

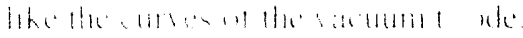

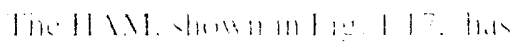

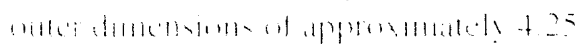

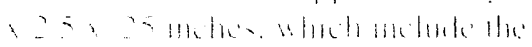

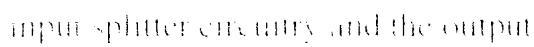

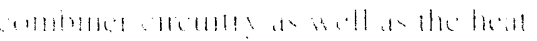

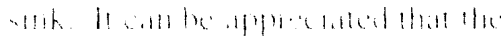

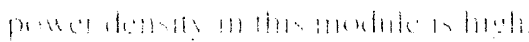

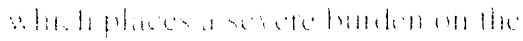

conling scheme. The conling xcheme

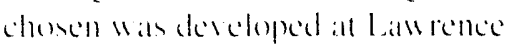
liscrmore Nallionall lahoratery and is called a microchamel heall sink. The Hamsistor chip is bonded (o) an aluminum nitride substrale. which is. in turn. honded to al silicon microchammel heal sinh. The heall sink has mant narrow grouser theregh which the coolant parses. The conling cappobility is serenly enhanced because the lins crealled hy the cutting process greatly increate the conting anceat and the widh of the chamnels ate lem than whe faminat lase thicksess of lla fluid parsing thrugh hem.

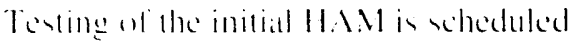

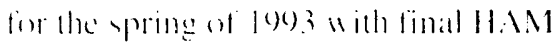
esing scheduled for the summer.

Sulecestul aperation af the

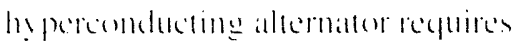
high purit aluminum wire. This wire

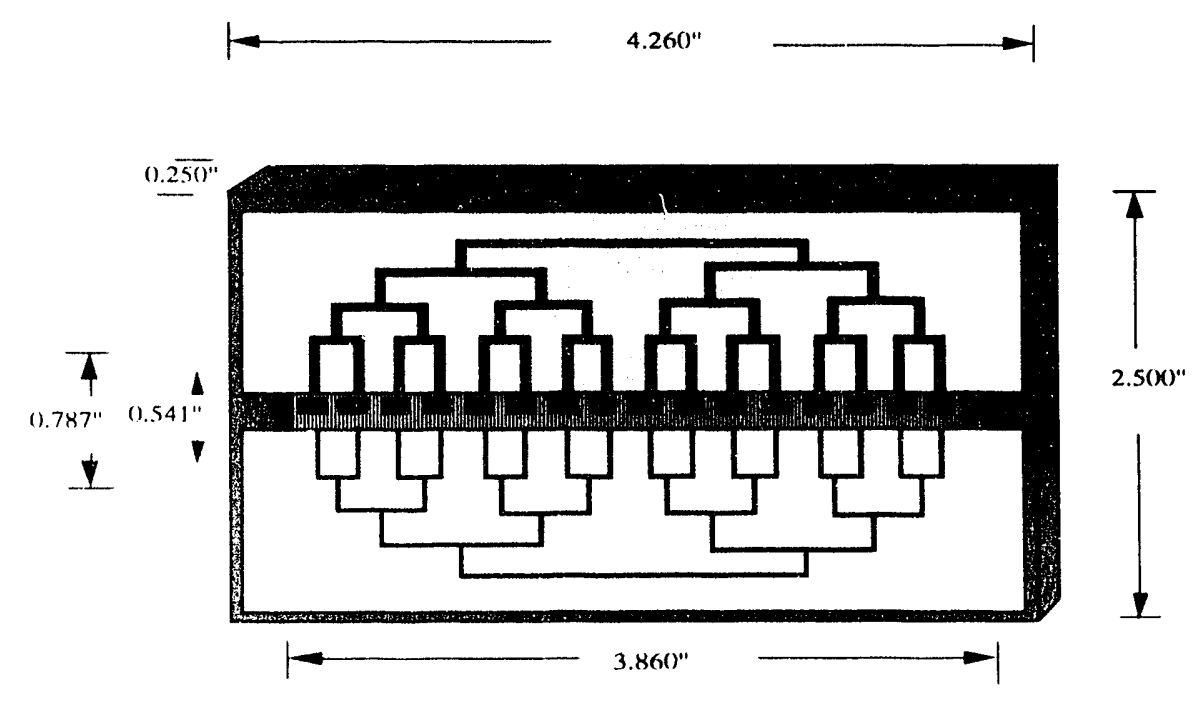

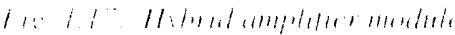

has been developed by Al. (O)A and they hase achieved a purity of (9) (4) 55 ;. When operatced at 20 K. the wire hat charalceristies similat to at superenderer without the troublesome problem ol yuenching. By using high purits aluminum wire in the comsertetion of the altermator. not only is wire meight salved. but losses are greatly reduced. which decreases the conling reyuirement. All these things reduce the size of the alternatlor. The current estimated specilic weight of an altemator using this technology is less that) $0.1 \mathrm{~kg} / \mathrm{kW}$.

AI COA has sucessifully fabricated 80() feer of rectangulat high purity wire: the wite is $0.16 \mathrm{in}$ square. Because high purily aluminum is very soft. the wire undergoes an involved fabricalion process that includes being draten. This repuires the wire to be chatd with a shell of Al-Fe-Ce for strength. There are many potential applications for this technology in transformer and rotating machinery design where supereonducting characteristics are desirable. (One need not une hỵdregen for centing: either neon or helium might be used. 


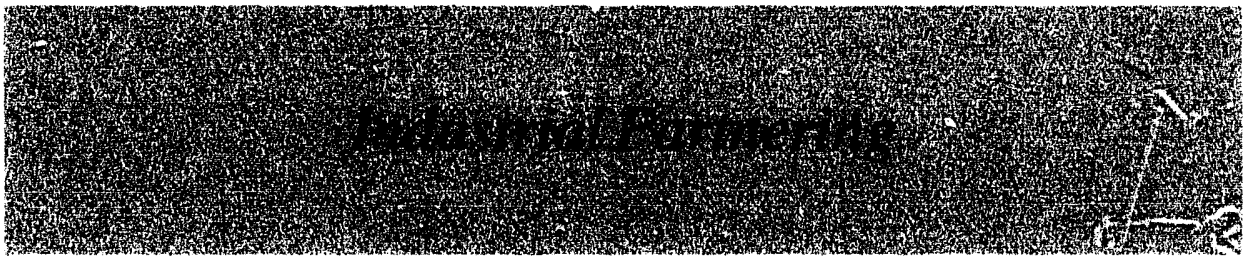

Over the past years, rapid and extensive changes in global politics have clearly indicated that weapons research is no longer a top national priority. National security and well-being are now more dominated by issues of economic competitiveness. These changes have caused AT Division to recast its business strategy so that greater breadth is added to the previously concentrated focus on accelerator-based advanced weapons systems. Examples of such programs are the Strategic Defense Initiative Organization (SDIO) support for missile defense systems using neutral particle beam (NPB) and free-electron laser (FEL). An opportunity to broaden our market for accelerator technologies has occurred through the Department of Energy's (DOE) and Defense Programs" sponsored Technology Transfer Initiative (TTI). This TTI was created as a "dual-benefit" program whereby the DOE supports Laboratory development projects that have aligned industrial support (i. e.. the industry supports its own personnel and facilities with "in-kind" resources project development activity). The dual-benefit realized is (1) industry is the beneficial recipient of the Laboratory's advanced technologies and competencies with the goal of becoming more competitive in the high-technology marketplace, and (2) the Laboratory is the beneficial recipient of transitional financial support allowing for development of new technology markets. Although the most visible. TTI is not the only avenue available by which to pursue industrial partnering. Additionally with the change in governmental administration, there will assuredly be increased incentive for the Laboratory to engage in industrial partnering as a way to strengthen economic competitiveness. This was reflected in the Laboratory Director's definition of industrial partnering as a strategic thrust areat that is projected to grow $1020 \%$ of the Laboratory's budget.
During FY 1992. AT Division hegan transitioning some of its projects to be more aligned with the commercial and industrial sector. Examples of such activities and identification of the relevant industrial partners follow.

EPICS: The Experimental Physies and Industrial Control System (EPICS) developed by AT- 8 has been united with Titan Industries and Tate Systems. They will adapt the EPICS software package to control problems in the commercial marketplace. ment: AT-5 joined with Varian Industries to commercially develop high-power, high-efficiency microwave klystrons for the communications marketplace (e. g., high-definition TV broadcasting).

Clearly the benefit to AT Division is a less costly microwave power source manufactured in the United States for future accelerator projects (e. g., Accelerator Transmutation of Waste |ATW and Accelerator Production of Tritium (APT|).

\section{Low-Level Radio-frequency (rf)} Controls: AT-5 has also found an industrial partner, Kinetic Systems, to market its low-level rf control units.

\section{Electron Becam-Plasma XUV Source:} Grumman Electronic Systems and Etec Systems, Inc., have joined with AT-7 to develop a unique extreme ultraviolet or soft $x$-ray (XUV) source with applications to semiconductor lithography.

\section{Microwanes for Chemical Processing:}

DuPont has paired with AT-9) to develop high-power microwave systems that facilitate chemical processing either by bulk heating or by inducing catalytic reactions.

\section{Klystrode Microwiave Tube Develop-}

Low-Energy Proton Accelerutor Syistems for Medical Applications: AT-1 has united with Neutron Technologies and more recently with Babcock and Wilcox to develop Boron Neutron Capture Therapy. Similar ties may also be created to develop commercial systems for proton therapy.

Frequency Agile and Compact FEL Systems: We are identifying programs to develop such FEL systems with Grumman Corporation as the industrial partner. The most noteworthy application for such a system is in naval ship defense in which the sensors of incoming cruise missiles will be defeatted.

Permanent-Magnet and Soft-Magnetic Material Development: AT-3, with Applied Materials Corporation, has proposed to develop advanced magnetic materials for use in accelerator structures and controls.

Plasma Processing S. Systems for Ion Implantation: AT-5 will supply the modulator and microwave systems needed by General Motors to develop ion-implantation on large volume and irregular surface objects (e. g.. car engine blocks).

Accelerator Based Conversion: This most ambitious program development has as its industrial partners Grumman, Westinghouse, and Bechtel Corporation. The activity involves marketing accelerator systems similar in performance to APT, but whose function is to drive a neutron source that converts weapons plutonium or other fissile material into a nonweapons grade isotope. An upgrade of such an accelerator based conversion (ABC) system could even "burn" the fissile material to produce energy.

The formation of these industrial partnerships does not guarantee that all will receive DOE funding. However, we expect the number of $\mathrm{A} T$-Division ties with industry for the purpose of commercializing accelerator technologies to increase in the coming years. 


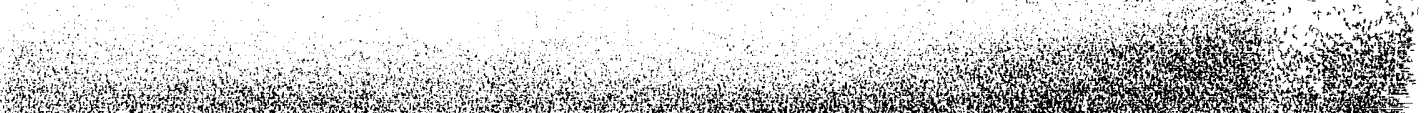

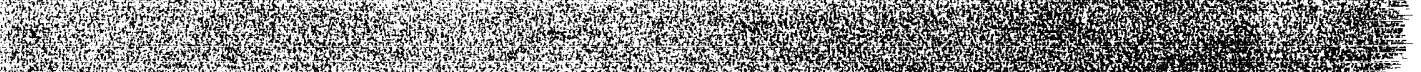

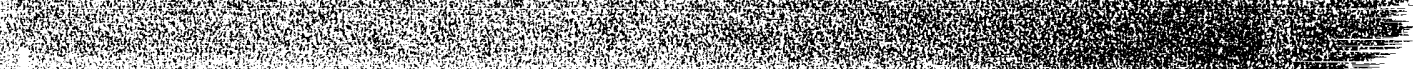

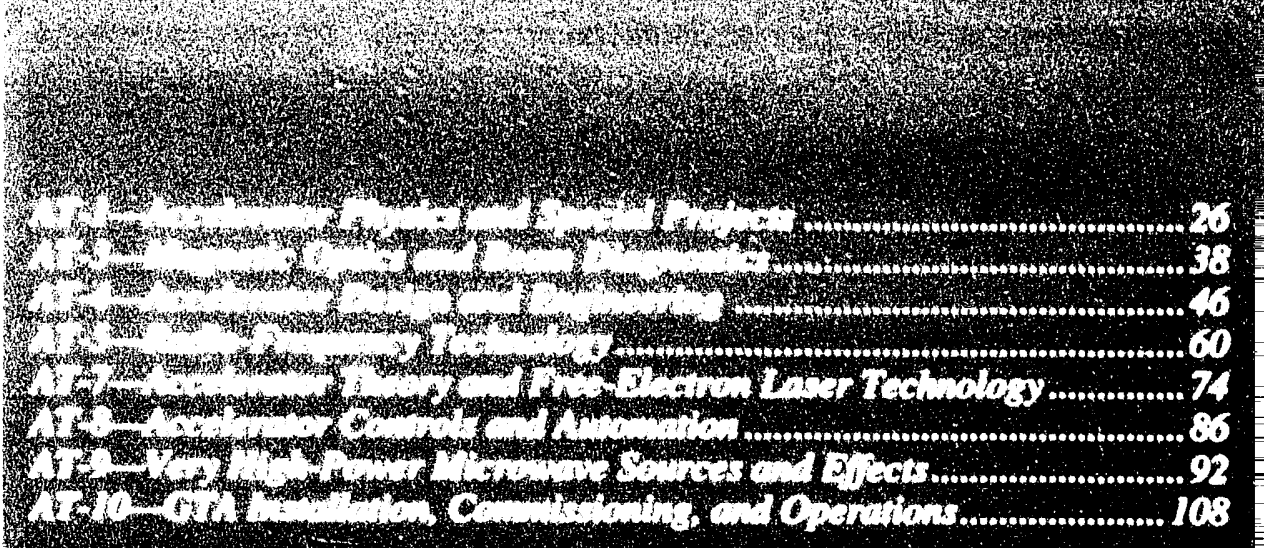

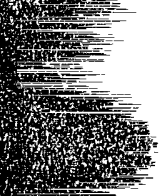

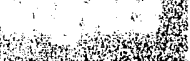

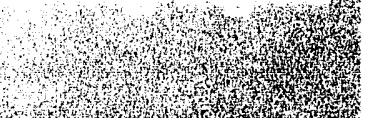

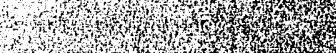
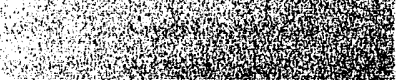

1.7.

1. $37^{7}$

1.

17.

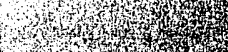

3.1.
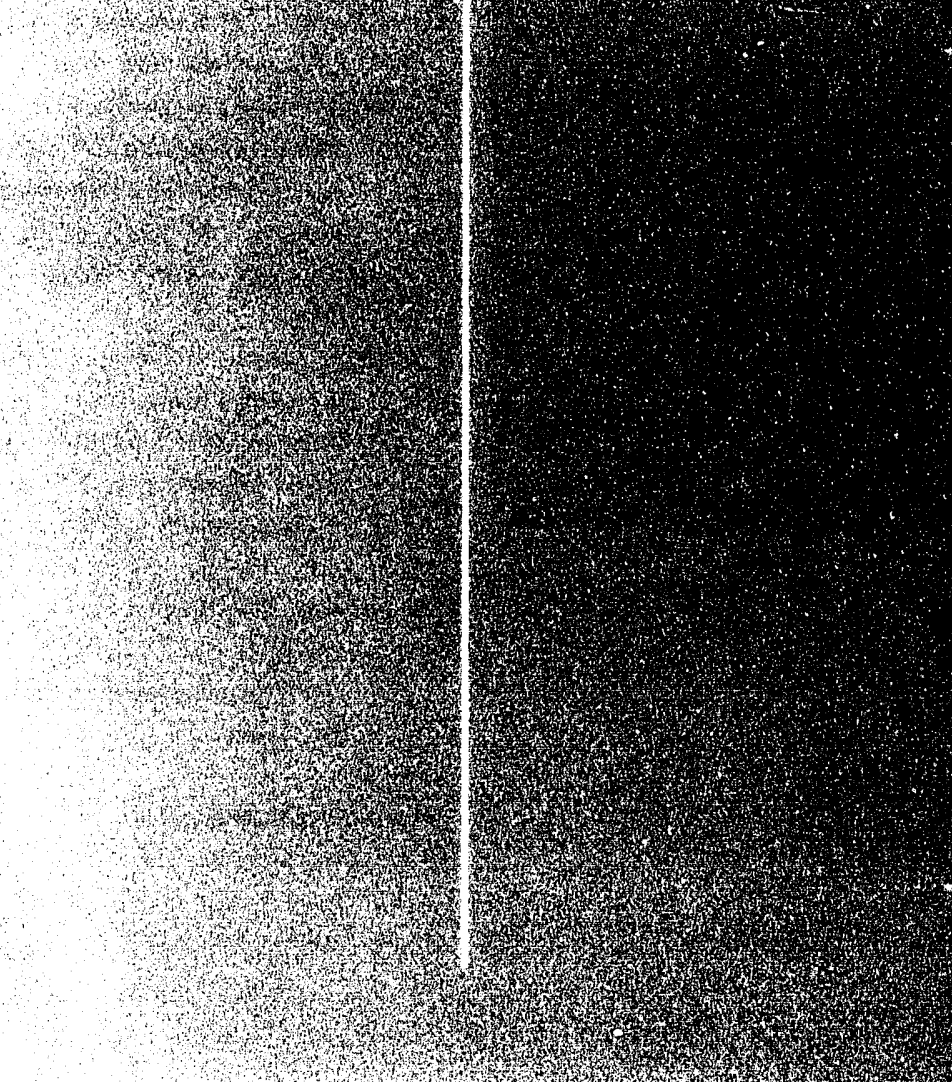

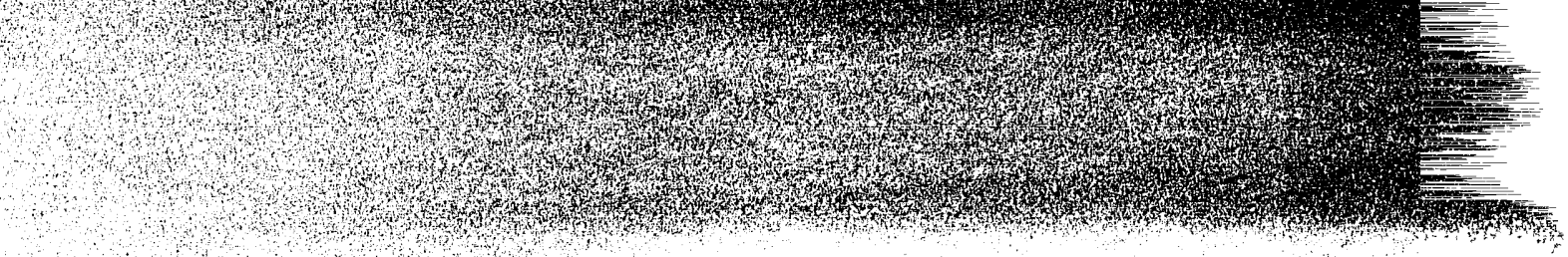




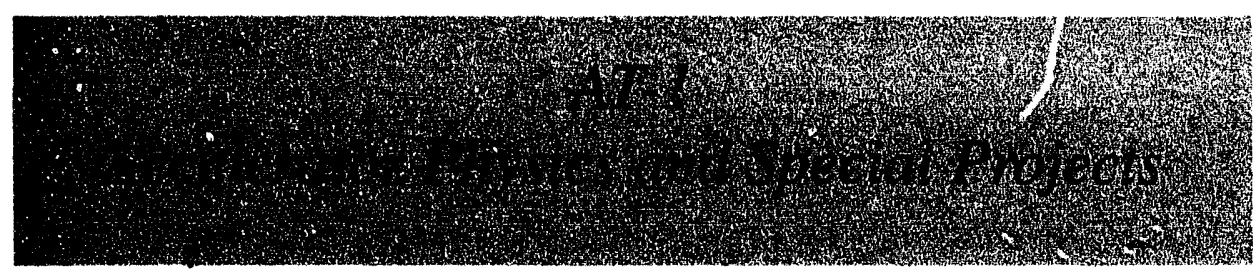

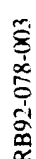
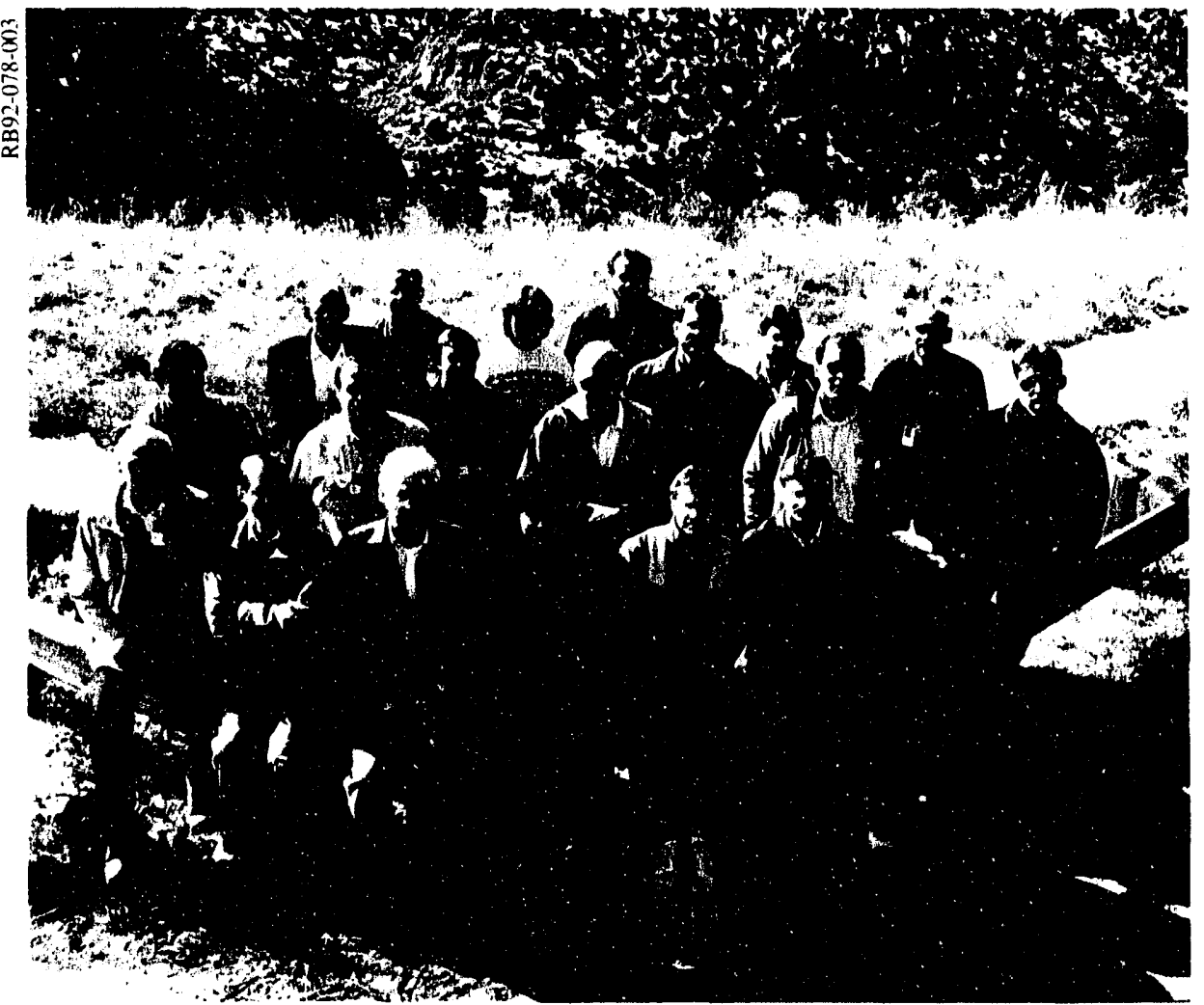

Introduction!

Accelerator Projects

Gironud Test Acceleranor Project

Sinplort

Photoinjector Lince for the Adranced Freer-Ele'tronl Lase't (AFEL)

Radio-Frecyuency Quadruprole Linac for the Supereonducting Super Collider.

Side-Compled Linac for the SSC.

Low-Poner Tuning of DTh. Cavities for the CTA

Radio Frequency Measure'me'mt and Analsisis Codes'

Beam Dinamics 29

Effects of ef Transients in the CCL for the SSC

Design and Simulation of a Bridge-Compled DTL for Accelerator Tramsmumation of Waste 31

Superconductivity Strong-Focusing $R F()$ .32

RFQ Linear Acrelerater for PET Isotope Productiom .32

POISSON/SUPERIISH Code Development for PC

comparibles 32

Structures Desedopme'mt

Laboratory .3 .3

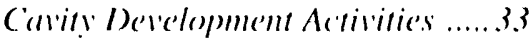

Achiche'me'tls.

Terehrinical Me'moranda .36 


\section{Introduction}

The AT-1 Group, Accelerator Physics and Special Projects, provides accelerator design and physics support for various AT Division projects. The staff at AT-I also pursues advanced topics in accelerator physics and technology. Additionally, AT- 1 builds small accelerators for special applications. What follows is a summary of the various AT-1 activities for the 1992 fiscal year.

\section{Accelerator Projects}

\section{Ground Test Accelerator Project Support}

The design of low-energy and highenergy end walls for the ten Ground Test Accelerator (GTA) drift tube linac (DTL) tanks was completed during FY 1990. These end walls are cryogenically cooled, brazed-copper units. The end walls for tanks five through eight were fabricated and tested during FY 1991. The remaining two units were completed during FY 1992.

\section{Photoinjector Linac for the Advanced Free-Electron Laser (AFEL)}

Under funding for the Experimental Research and Development Initiative and in conjunction with AT-7, we completed a $1300-\mathrm{MHz}, 20-\mathrm{MeV}$ photoinjector linac for the Advanced Free Electron Laser (AFEL). This unit (Figs. 2.1 and 2.2) is now in laboratory service. In many ways, the AFEL linac is similar to both the 6-MeV high brightness accelerator FEL (HIBAF) (FY 1989, now APLE prototype experiment [APEX]) and the $6-\mathrm{MeV}$ National Center for Laser Research (NCLR) (University of Twente. FY 1991) photoinjector electron linacs. Like them, it is a compact. brazedcopper, on-axis, coupled, radio frequency (rf) structure. It employs the same photocathode hardware as APEX, so that they may eventually share photocathode production and transport systems.

The AFEL linac differs from its two predecessors primarily in final energy (20 MeV, 11 accelerating cells versus 6 $\mathrm{MeV}, 6$ accelerating cells) and in its capability for cryogenic operation. The system can be cooled with either liquid nitrogen or water. The copper rf structure is thermally isolated from its surrounding vacuum vessel, allowing in situ $\mathrm{rf}$ baking to achieve the high vacuum $\left(<1 \times 10^{-9}\right.$ torr $)$ required by the cesiated photocathode. At temperatures approaching $77 \mathrm{~K}$, the electrical resistance of the copper is expected to be significantly lower than at conventional operating temperatures, producing a quality factor $(Q)$ enhancement of about 2. In addition, the thermal expansion coefficient is lower and the thermal conductivity higher at those temperatures. These three factors contribute to the possibility of a much higher rf duty factor than is otherwise possible without direct nose cooling. More subtle improvements have been made in the form of larger focusing and bucking solenoids and in the number and orientation of the coupling slots that connect the accelerating cavities to the on-axis coupling cavities. These improvements are expected to result in the brightest electron beam source yet.

The photoinjector linac was delivered in FY 1992 and has been operating at ambient temperature. Tests of cryogenic operation are planned for FY 1993.

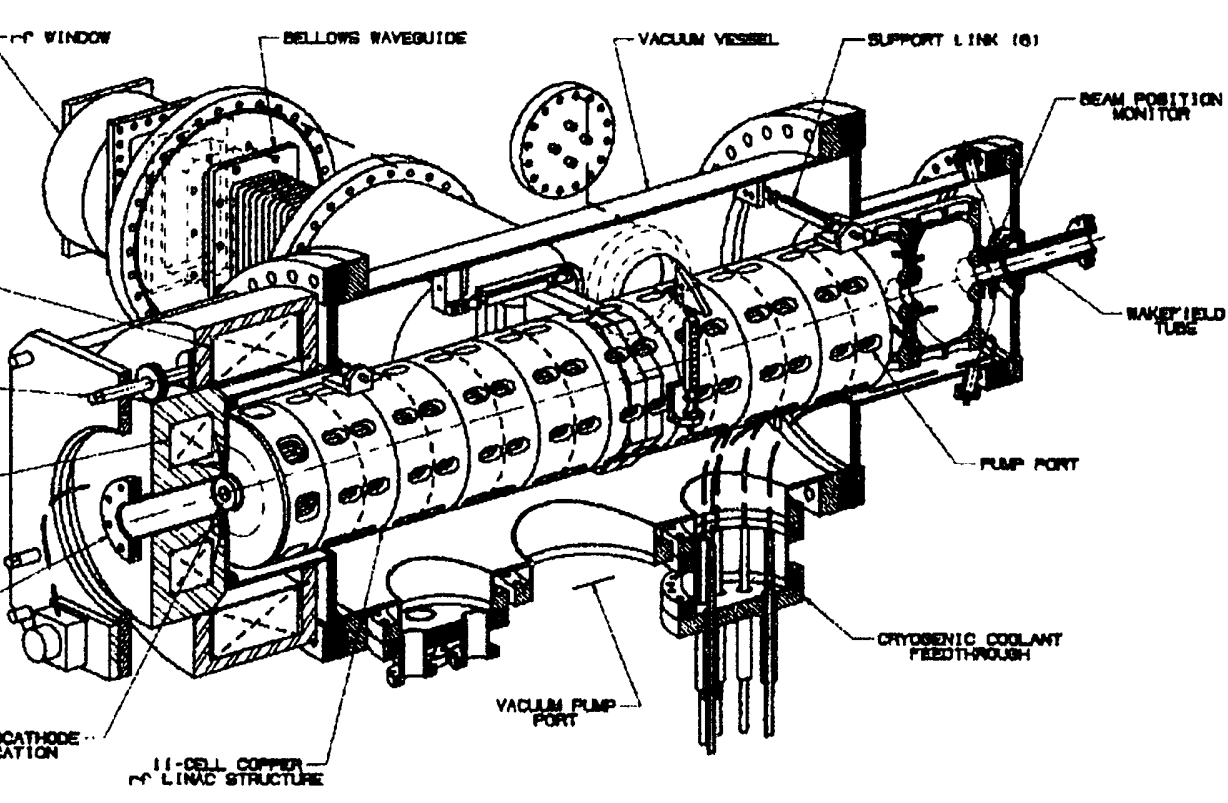

Fig. 2.1. Schematic of 1300-MH-, 20-MeV crvogenic linac for the AFEL.

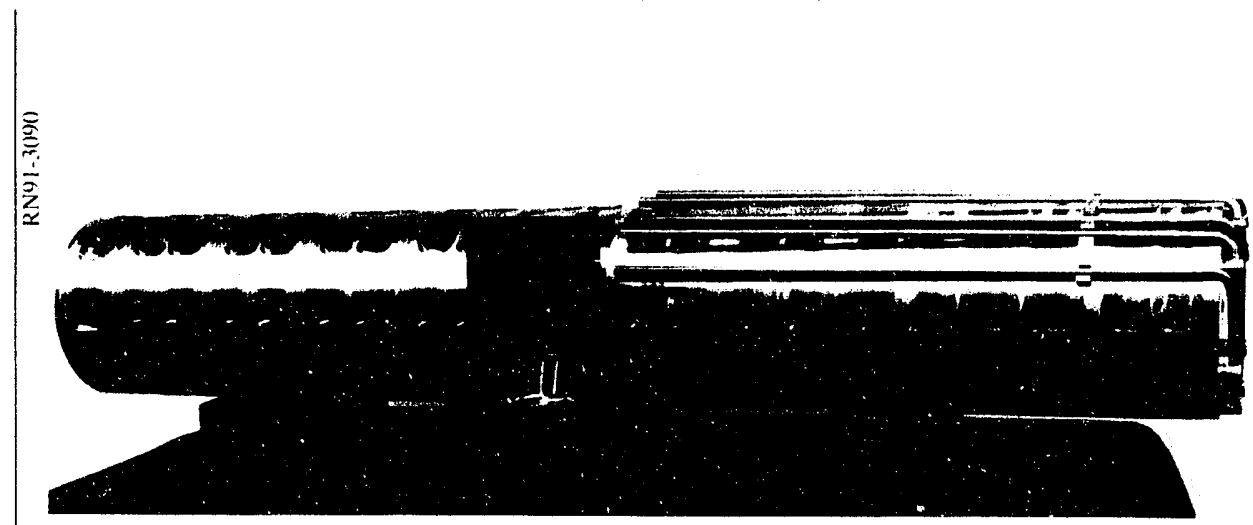

Fig. 2.2. Brazed copper, on-axis, compled linac for the AFEL. 


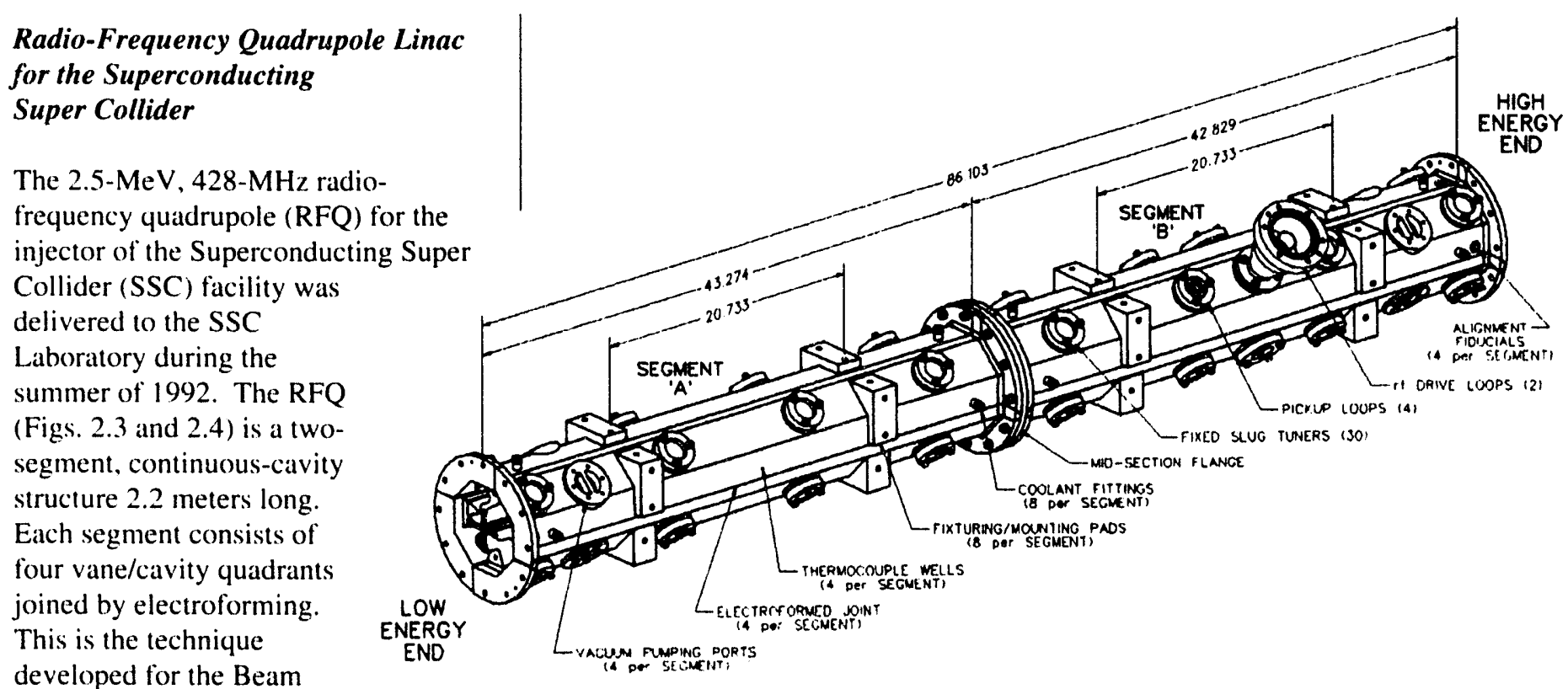

Experiment Aboard a

Rocket (BEAR) RFQ and was also utilized for the Continuous-Wave Deuterium Demonstrator (CWDD) RFQ. It was manufactured jointly by LANL and GAR Electroformers of Danbury, CT.

\section{Side-Coupled Linac for the SSC}

We are carrying out the detail mechanical design for the side-coupled linac (SCL) (Fig. 2.5) for the SSC. It is a 1284- $\mathrm{MHz}$ structure consisting of nine modules, each with eight accelerating tanks ( 72 total) joined by bridge couplers (63 total). This is a graded- $\beta$ structure and will be described on 1400 mechanical drawings. The drawings are being produced by a CAD programming technique that allows the physics dimensions of the accelerator and bridge tanks to be read from data files so that the detail and assembly drawings can then be generated. This method will provide considerable savings in cost and time. The SCL will be a brazed-copper structure, fabricated in the Peoples' Republic of China.

Fig. 2.3. Schematic of the 2.5-MeV, 428-MHz radio-frequency quadrupole $(R F Q)$ linac for the superconducting super collider (SSC).

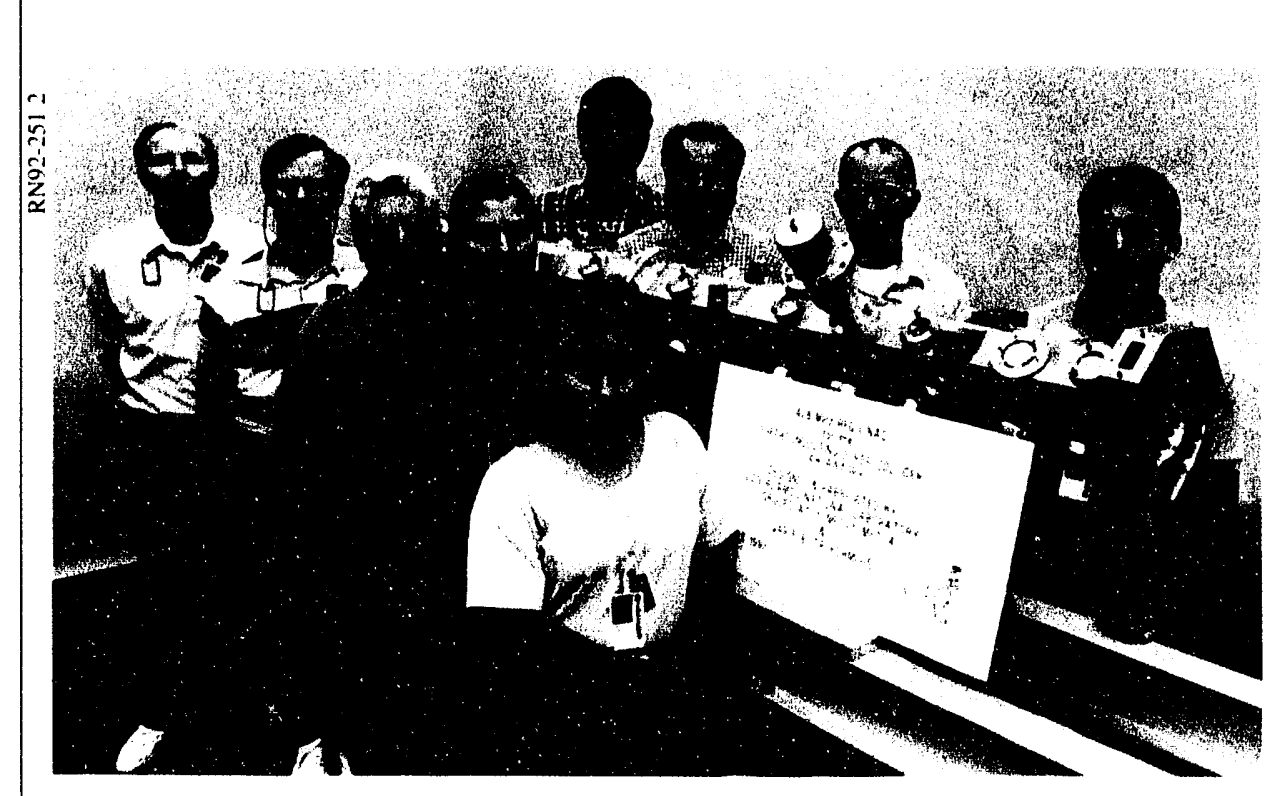

Fig. 2.4. Final assembly of 2.5-MeV. 428-MHz RFQ linac for the SSC (front-left: Dale Schrage. Lloyd Young, Phillip Roybal, Felix Martines. Angela Naranjo; rear-left: William Clark, Roy DePaula, Jim Billen, Doug Aikin). 


\section{Low-Power Tuning of DTL Cavities for the GTA}

We have tuned the first five of ten 850$\mathrm{MHz}$ DTL cavities for the GTA.

Tuning included low-power measurements of the following: ramped field distribution, field stability, cavity Q, and coupling to the $r f$ power source. Before post couplers were installed, the unstabilized field distributions agreed with predictions based upon SUPERFISH code calculations and applications for the Slater perturbation theorem. We adjusted and stabilized the relative field distributions to within $\pm 0.25 \%$ of the design ramp. The lowpower measurements also included the $\mathrm{Q}$ enhancement and the field distribution at cryogenic temperatures. There were no significant changes in the relative field distribution at cryogenic temperature. The room-temperature $\mathrm{Q}$ was typically $75 \%$ to $85 \%$ of the SUPERFISH Q, and the cryogenic Q was about 3.2 times the room-temperature $\mathrm{Q}$.

\section{Radio Frequency Measurement and Analysis Codes}

We have developed a comprehensive collection of computer codes for measuring, analyzing, and displaying field distributions in rf cavities. Development work began in 1984 for 8086-based computers. The codes now run on 486 and 386 PCs and include extensive on-line documentation. The programs BEADPULL and QUADPULL collect frequency shifts as a metal or dielectric bead traverses a resonant cavity at constant speed. QUADPULL measures fields in all four quadrants of RFQ cavities. Analysis codes apply the Slater perturbation theory to convert frequency shifts to fields. BEADPLOT plots the field distributions and calculates field integrals. DTLPLOT analyzes and plots the results of axial BEADPULL measurements in DTLs or coupled-cavity linacs $(\mathrm{CCL})$. QUADPLOT does the same for QUADPULL data in RFQs. Supporting programs such as BEADPLOT, DTLPLOT, and QUADPLOT prepare design data for comparison with the measurements and compute averages of multiple measurements. Program COUPLING extends the accuracy of an impedance analyzer for $Q$ and voltage standing wave ratio (VSWR) measurements. The COUPLING code plots reflection coefficients on a Smith chart and analyzes the resonance circle to get $\beta$.

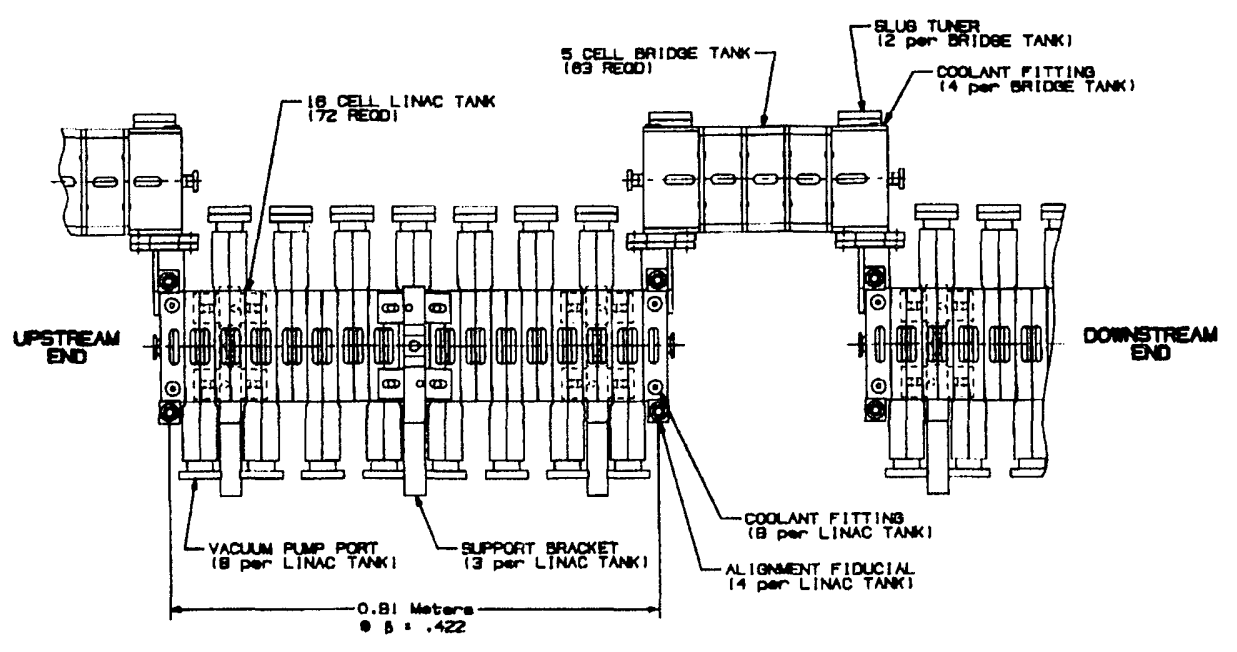

Fig. 2.5. Mechanical design for the side-coupled linac (SCL) for the SSC.

\section{Beam Dynamics}

\section{Effects of $r f$ Transients in the $C C L$ for the SSC}

Each module of the CCL for the SSC consists of 8 tanks with 16 accelerating cells each. The tanks are coupled with bridge couplers. The rf power drive is in the center of the module at the bridge coupler between the fourth and fifth tanks. In simulations of the beam dynamics, the if power is turned on $10 \mu \mathrm{s}$ before the beam is turned on. This time lapse allows the fields to build up and stabilize before they are required by the beam. When the beam is turned on, the beam loading causes the fields to change. This transient state of the fields, together with their effect on the beam was studied. We developed a model to calculate the field distribution throughout the module as a function of time. We then ran beam dynamics simulations with the results of this model corresponding to different times during the macropulse, deriving an estimate of the effect of the transients from the results of these simulations.

The code TRAN6 calculates the field amplitude and phase of all the accelerating cells versus time. TRAN6 displays a moving picture of the amplitude of all the accelerating cells, a history of the forward power, the average amplitude of all the accelerating cells, and the reflected power. The amplitude of each accelerating cell and coupling cell is shown by the small asterisks. The scale for the amplitude is shown on the left side of the plots, and the scale for the forward and reflected power is on the right side. The time scale is on the top of the plots. The scale on the bottom shows the accelerating cell number for the plot of amplitude versus accelerating cell number at the current time, which is displayed at the top. 
In Fig. 2.6 the time has advanced to $10.75 \mu \mathrm{s}$. The beam was turned on at 10 $\mu \mathrm{s}$. The amplitude in the tanks has dropped 1\% to $2 \%$. The rf power has increased because the feedback system is requiring more power to compensate for the beam loading. The if power scale on the right side of the plot means percent of maximum klystron power. The simulation had proportional, integral, and differential (PID) feedback built into the code. This PID feedback on the if power simulates a nearly ideal PID feedback control system: however, no feedforward that may be used on the SSC-CCL of control system is included.

For beam dynamics simulation, we renormalize the design values $E_{d}$ of the accelerating electric field in any given lank by the ratio of the transient field amplitude levels $E_{\text {i }}$ in the tanks, and the steady-state values of $E$, just before $t=10 \mu \mathrm{s}$. The beam loading is assumed to be $12 \%$, an average for the nine modules for a beam current of $25 \mathrm{~mA}$. In the simulation illustrated by Figs. 2.6 and 2.7, we ignored the very small field amplitude tilt from cell to cell within a tank and took the average value as the field amplitude for the entire tank. Particle simulation runs were made for 1000 macroparticles transported through the entire CCL at several $t$ values, before and alter $t=10$ $\mu$ s. Figure 2.7 shows the energy protiles at $t=9.95 .10 .5,10.75,11.00$, and 12.00$) \mu \mathrm{s}$, respectively.

As expected, the size of the transverse root-mean-square (rms) beam (not shown) during the transient stage does not change when compared with the steady state sitlation. However, the transient fluctuation in Ea causes phaseenergy oscillations in the longitudinal phase-space, an shown in Fig. 2.7. The mean energy $W$, of the output distribution deviates significantly from the steady-state value within the first microsecond after $t=10.0 \mu \mathrm{s}$. At $t=10.75 \mu \mathrm{s}$, the mean energy deviation is $=5.59 \mathrm{keV} . \mathrm{Al} t=11.50 \mu \mathrm{s}$ the conditions are virtually the steady state situation. No particles are lost at any

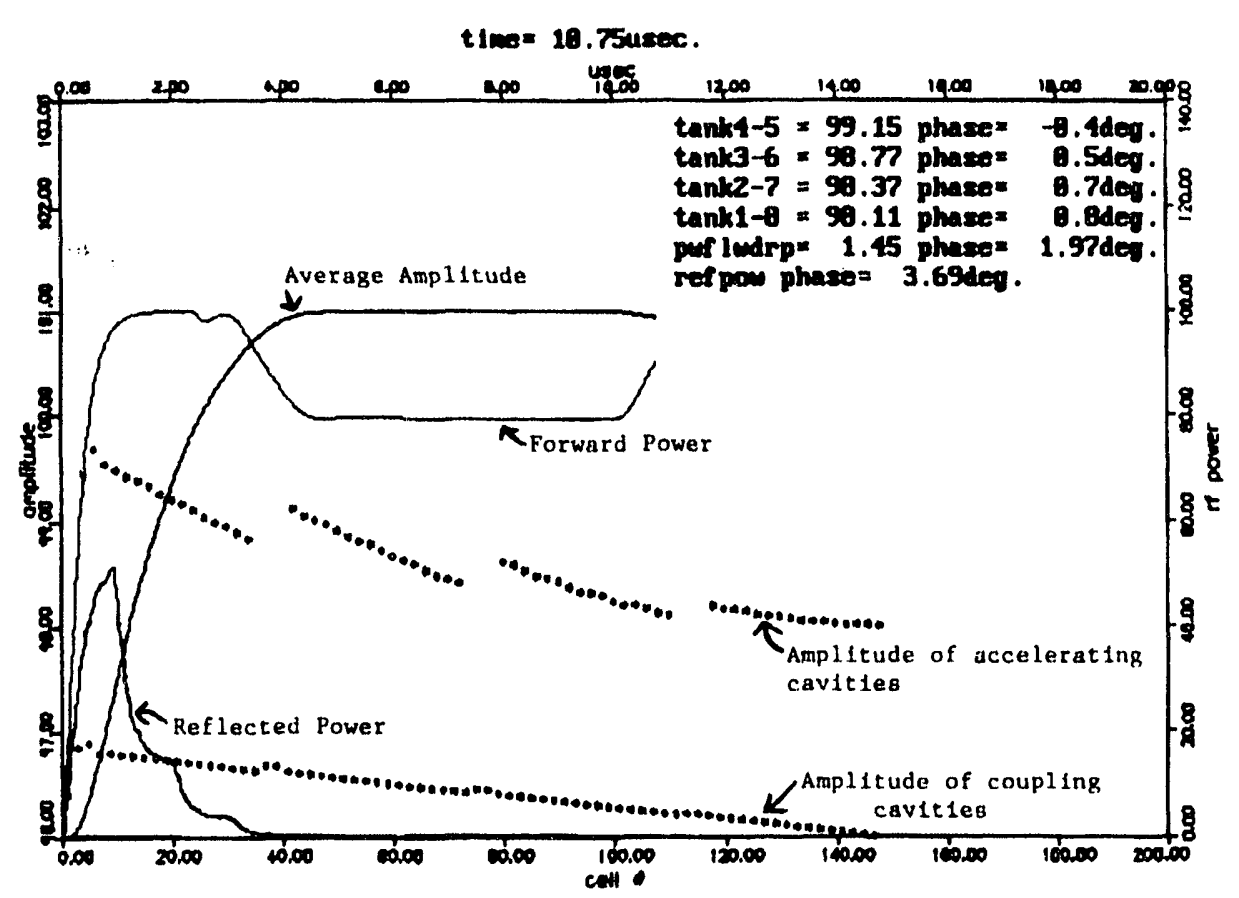

Fig. 2.6. TRANG display' at time $=10.75 \mu \mathrm{s}$.

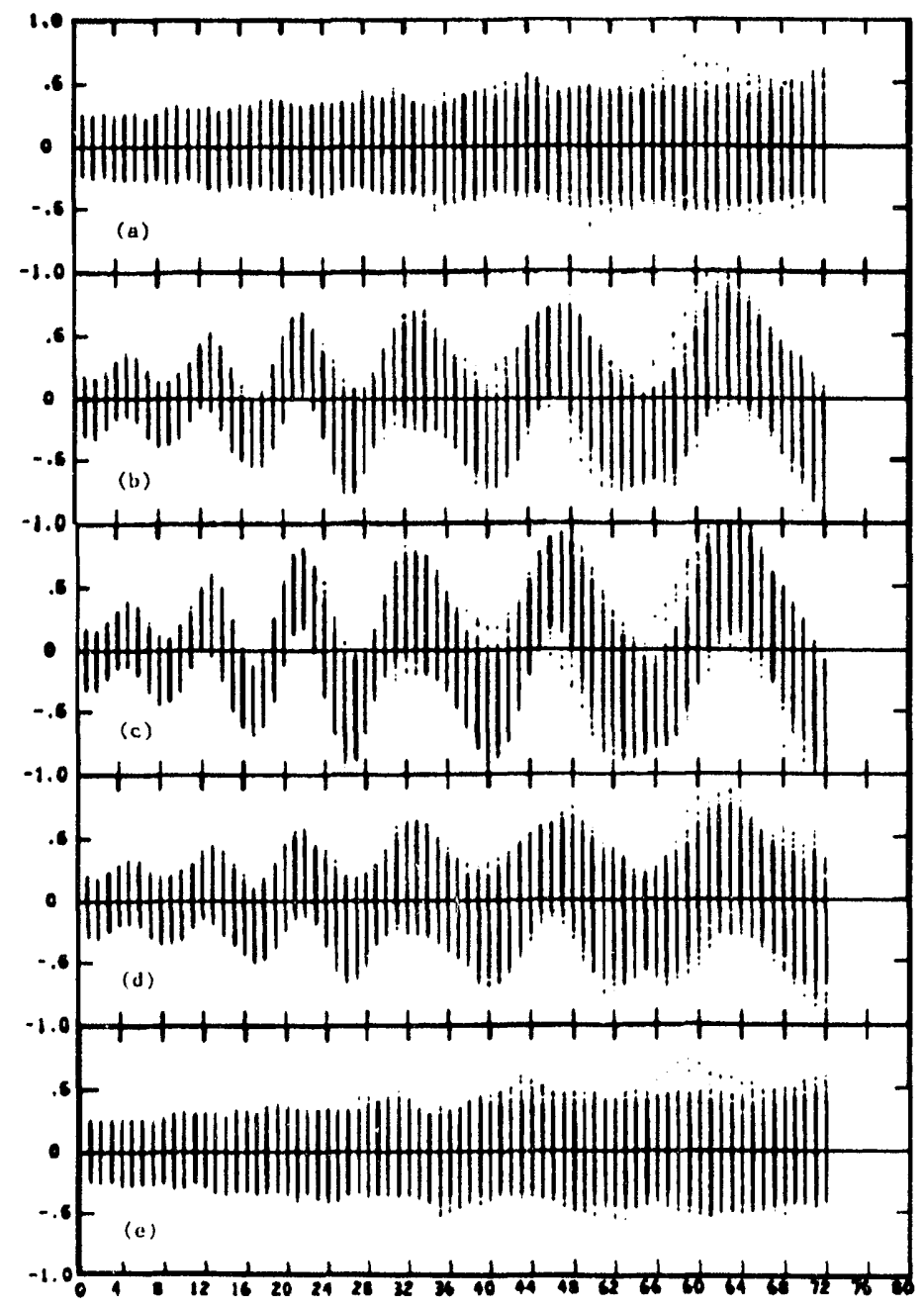

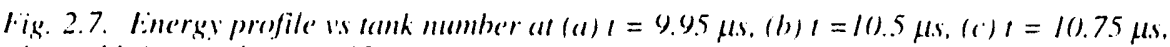
(d) $t=11.0 \mu \mathrm{s}$ (ind $($ (c) $)=12.0 \mu \mathrm{s}$. 
time because of the transient effect. There is also no statistically meaningful emittance growth during the transient stage. The deviation in the Phase is not significant.

\section{Design and Simulation of a Bridge- Coupled DTL for Accelerator Trans- mutation of Waste}

Recent Los Alamos designs of highcurrent, continuous wave (cw), proton linacs for accelerator transmutation of waste (ATW) incorporate beam funneling to achieve desired levels of current, emittance, and rf efficiency in the high-beta accelerating structure. Typical designs have a front end consisting of two 350-MHz linacs, each composed of an RFQ and a DTL, which accelerate protons to $20 \mathrm{MeV}$. The two beams are funneled into a 700$\mathrm{MHz} C \mathrm{CL}$ that has a final energy in the range of 800 to $1600 \mathrm{MeV}$. The design choice for a $700-\mathrm{MHz}$ accelerating structure in the 20 to $80 \mathrm{MeV}$ region would conventionally be an Alvarez DTL with permanent-magnet quadrupoles (PMQs) in the drift tubes. However, for high-current cw applications, the threat of radiation damage from beam loss makes the use of PMQs undesirable, and $700-\mathrm{MHz}$ drift tubes cannot accommodate electromagnet quadrupoles (EMQs). The initial design approach was to begin the 700$\mathrm{MHz}$ CCL structure at $20 \mathrm{MeV}$.

Recent engineering analysis has shown that fabrication of such a structure at low-beta values would be complicated and power efficiency would be low. We have derived a modified DTL concept, the bridge-coupled DTL (BCDTL), that provides an attractive solution for the problem region, with simpler fabrication and higher efficiency. Figure 2.8 shows a schematic diagram of an ATW-type machine. The funneling energy is set at $20 \mathrm{MeV}$ because of increased engineering complexity; stricter beam dynamics requirements at higher energies, i.e., higher deflector fields and increased number of funnel components; and the desire to minimize beam-loss-induced activation at the transition region. The ratio of transverse aperture to $\mathrm{rms}$ beam size has been used as a figure of merit in estimating beam losses. In order to minimize beam loss, we have required this ratio to be 6 or larger above $20 \mathrm{MeV}$ throughout the linac. This condition requires adequate transverse focusing throughout the linac to maintain beam size and to minimize emittance growth, which could produce beam halo. The ratio of aperture to rms beam size is dependent on the intertank spacing and the type of focusing lattice used. The number of accelerating cells per tank of the

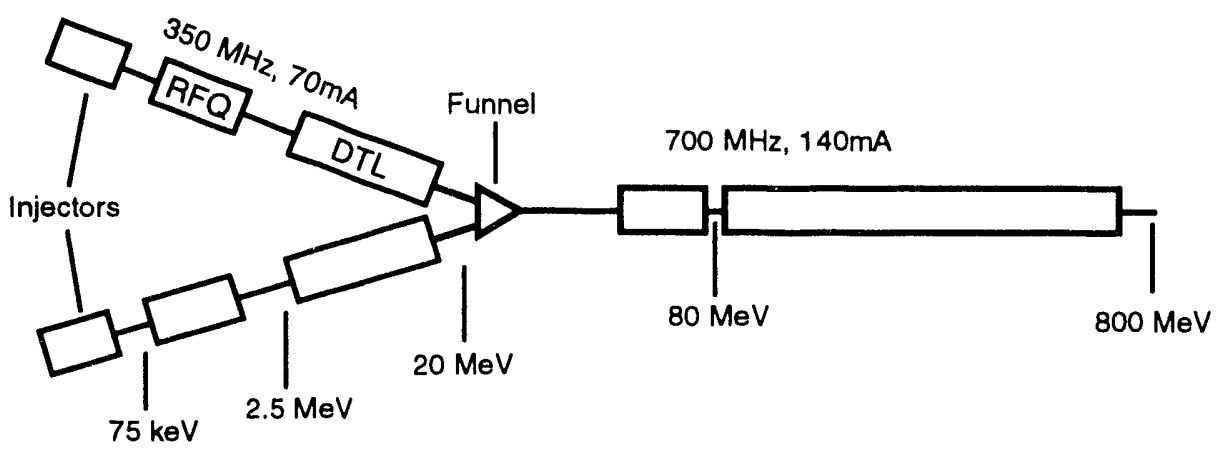

Fig. 2.8 Schematic diagram of an ATW-type accelerator. The BCDTL would be wsed to accelerate the beam from 20 to $80 \mathrm{MeV}$ after the beam has lefi the fiumel.

\section{0-MeV Bridge Coupled Drift-Tube Linac Module (700MHz)}

\section{Weight: Approx $3000 \mathrm{~kg}$}

Coolant flow: $100 \mathrm{gal} / \mathrm{min}$

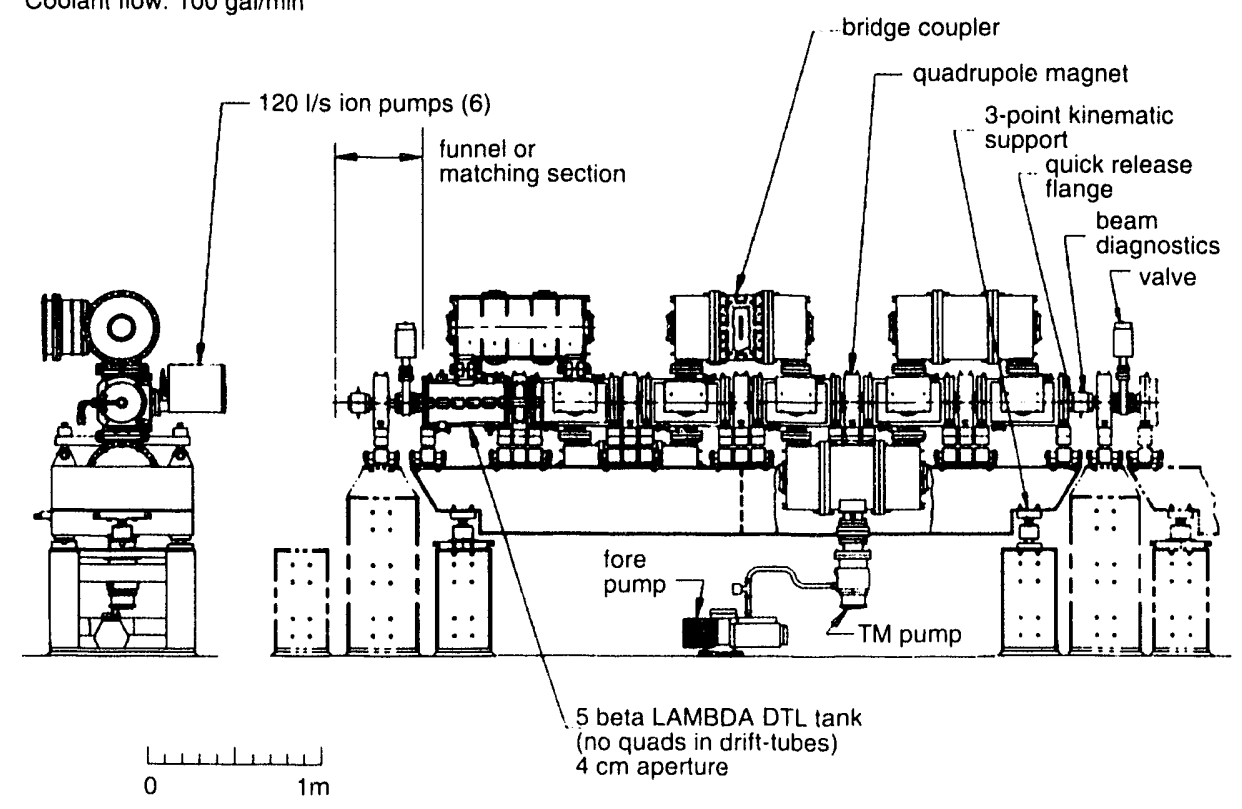

Fig. 2.9. Conceptual engineering drawing of what a four-fumk module' of the BC'DTL. might look like.
BCDTL and the transverse phaseadvance per focusing period were chosen to optimize the transverse ratio of aperture to rms beam size.

Using a modified version of the PARMILA code, the BCDTL shown in Fig. 2.9 was simulated from 20 to $80 \mathrm{MeV}$ as 86 individual tanks with intertank quadrupoles for transverse essentially no transver'se emittance whe longitudinal emittance, however, grows because of the constant accelerating gradient. This focusing. In this situation there is 
constant accelerating gradient allows the longitudinal focusing per unit length to become weaker as a function of distance along the linac. Nonetheless, there appears to be no effect of longitudinal emittance growth on the transverse emittance. Such an emittance growth could result in a degradation of the ratio of the aperture to $\mathrm{rms}$ beam size.

The results of this study indicate that the BCDTL could be a practical alternative to a CCL for the 2()- 10 100)$\mathrm{MeV}$ energy region of an ATW accelerator. This type of structure provides adequate transverse focusing of high-current heams as needed to control beam-loss-induced structure activation and is electrically efficient in this beta regime.

\section{Superconducting Strong-Focusing $R F Q$}

Our objective is to develop a niobium. superconducting, RFQ particle accelerator for high-field operation. This development would enable us to build very compact low-velocity of ion linacs with energies up to ahout $10 \mathrm{MeV}$ for protons and with the capability for acceleratting high-beam currents. The project represents a new application for rf superconducting accelerators.

Our approach is to (1) determine the best parameter choices for the design of high-field superconducting proton RFQs: (2) identify the superconducting $R F($ applications that appear to be most attractive, especially those that represent a good entry point for at phased development of the technology: (3) design and construct a prototype supereonducting 4-vane calvity, and operate it at the highest fields possible; and (4) increase our understanding of the beam physics of high-current RFQ accelerators in order to enable us to lake maximum advantage of the new technology we are developing.

Progress during the past year includes (1) RFQ heam-dynamies design studies that include the design, using a new procedure, of an $11-\mathrm{Mc} V \mathrm{RFQ}$ for radioisotope production for positronemission tomography (PET): (2) completion of the cavily design for the 30-cm long niobium prototype cavity: (3) establishment of the mechanical design (Fig. 2.10) for the prototype cavity: (4) fabrication of the niobium cavity parts and stainless-steel fixtures for an electron-beam weld test: and (5) basic beam-physics studies, including measurements and analysis of the output beam from the world's highestintensity RFQ at the European Center for Nuclear Research.

\section{RFQ Linear Accelerator for PET Isotope Production}

We have developed a new concept for a compact $11-\mathrm{MeV}$ linear accelerator in a single 3-m RFQ structure. This new concept should be an attractive choice for a radioisotope-production accelerator for the PET application. We have investigated two options: (1) a normalconducting copper RFQ, that would operate in a pulsed mode and (2) a superconducting $R F Q$ thall would operate in a continuous $(100 \%$ duty fictor) mode. The design concept includes a new approach, based on the recent $\mathrm{RFQ}$ that was built for the SSC application, that uses a ramped voltage to maintain high accelerating fields throughout the structure. This results in at very compact alcelerator. Beamdynamics calculations for an unoptimized design demonstrate the basic feasibility of the idea. We are also fortunate that, at this frequency, the rf power sources are commercially available for both the normal and superconducting calses. The next step would be to produce an optimized design and build a prototype accelerator to demonstrate the concept.

\section{POISSON/SUPEREISH Code Development for PC Compatibles}

We have adapted the POISSON/ SUPERFISH codes to run on 486 or 386 PCs. The PC version includes features not found in the standard version. These extra features include (1) control programs for atutomatically tuning RFQ. DTL. and CCL cavities; (2) a complex version of the of field solver FISH: (3) memory allociation for temporary arrays; (4) many line regions for dividing the mesh into fine or coarse sections; (5) full support for multiple-cell DTL cavities; (6) plot files for displaying resonance-search and transit-time data: and (7) on-line documentation. We modified AUTOMESH extensively to generate self-consistent logical and physical coordinates. This new, more-robust code greatly reduces the number of crashes in LATTICE crashes that previously were caused by ill-formed mesh triangles along boundaries. Program FISH detects and corrects potentially poor placement of the driving point. Programs AUTOMESH, LATTICE. FISH, POISSON. SFO,

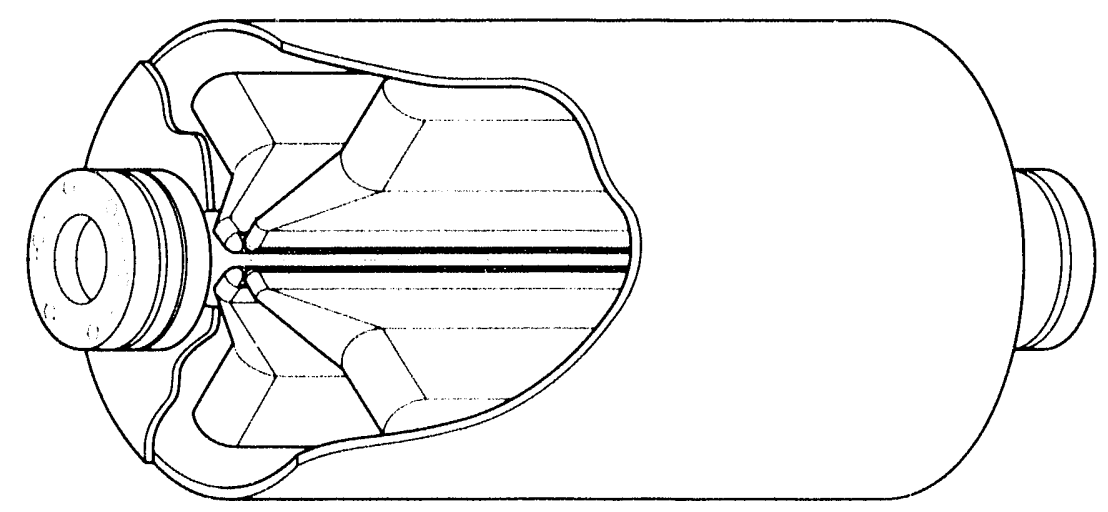

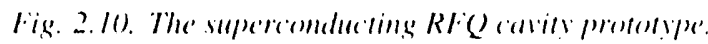


and VGAPLOT return DOS exit-error codes for use by control programs. The DOS exit-error codes solve arbitrarily large problems, limited only by computer resources. Each program allows free format entry of CON array elements, and provides error checking of the user entries. Our new root finder and convergence criteria have been implemented in release 4 of the standard version. We distribute the PC version of these codes to users in the accelerator community upon request.

\section{Structures Development Laboratory}

\section{Cavity Development Activities}

At the structures laboratory we concentrate on superconducting accelerator cavity development. This effort is directed at developing the database and technical expertise required to design a superconducting proton accelerator. Earlier work had identified pion acceleration as a programmatic goal. Changes in programmatic emphasis have given top priority to proton acceleration. All of the previous design and database work can be applied to the proton goal.

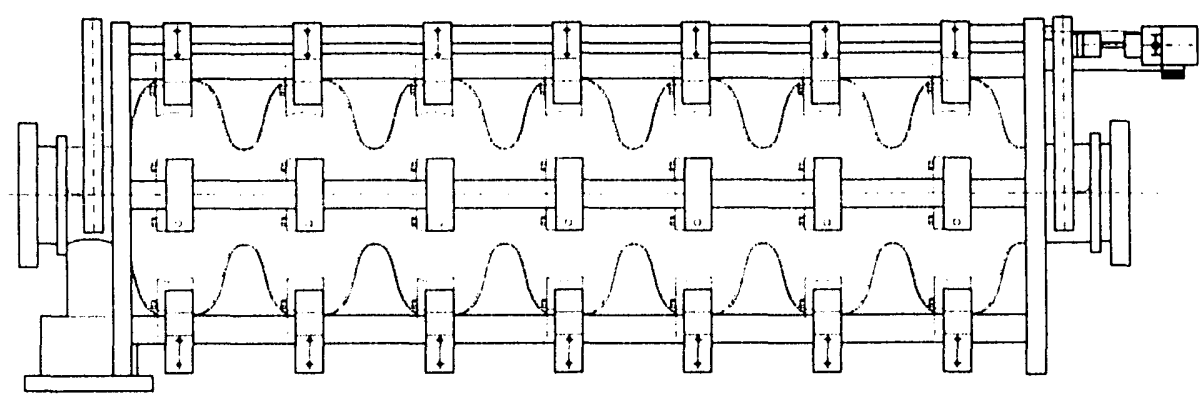

Fig. 2.11. Prototype design drawing for a superconducting seven-cell cavity for ion acceleration. c'mphasizing a mechanically rigid structure to reduce microphonics, field flatmess upon cooldown to $5 \%$, and high accelerating gradients.

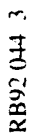

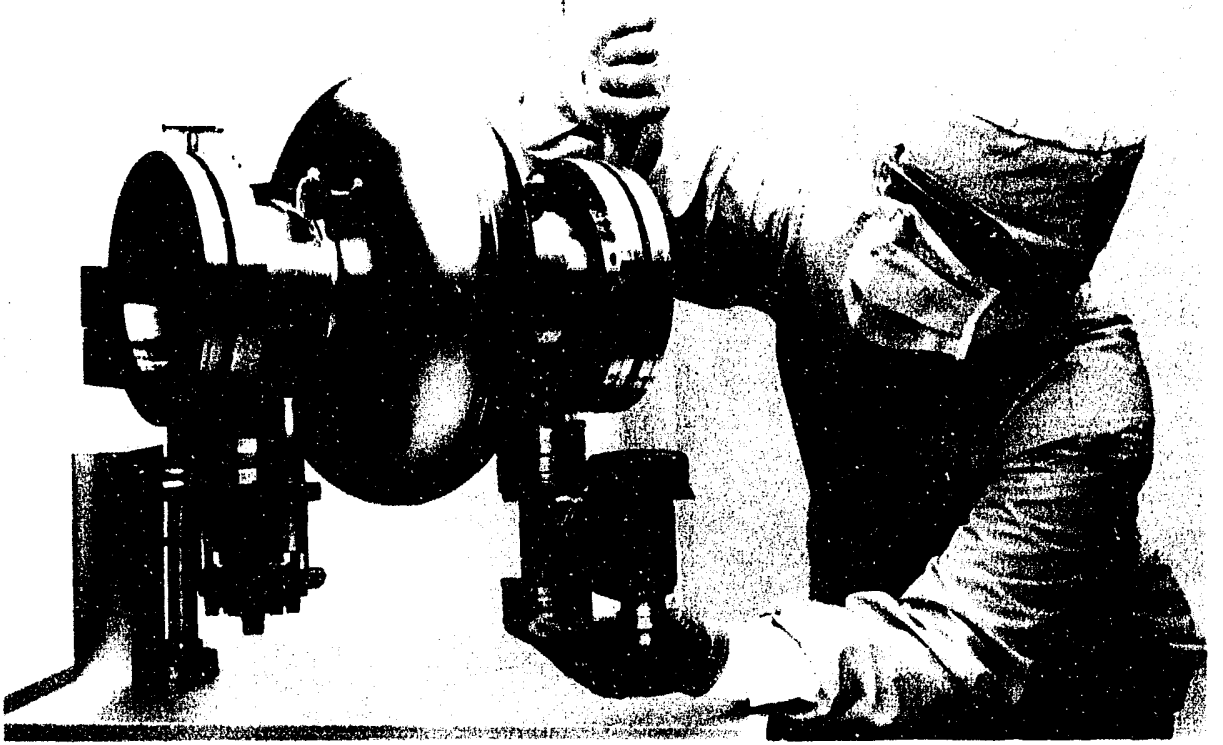

Fig. 2.12. After clean-room assembly, an 805-MHz- cavity is ready for testing in $2 \mathrm{~K}$ helium superfluid liquid. (RB92 (0440.3)
The design frequency of the accelerating structure matches the Los Alamos Meson Physics Facility (LAMPF) frequency of $805 \mathrm{MHz}$. In Previous fiscal years we concentrated our work on smaller $3-\mathrm{GHz}$ cavities in order to establish an initial database and develop cost-effective handling techniques. This fiscal year we concentrated on testing single-cell 805$\mathrm{MHz}$ cavities. We have achieved average and maximum values for peak surface electric fields of $30 \mathrm{MV} / \mathrm{m}$ and $50 \mathrm{MV} / \mathrm{m}$, respectively. These are world class results, exceeding previously reported results at this frequency. The values are consistent with the design goals for the program, namely, a cavity average of $20 \mathrm{MV} / \mathrm{m}$ for the surface electric field. A next major step in the program is a test of a sevencell, 805-MHz-cavity structure, complete with a stiffening structure for reducing microphonics, and a tuning fixture. The cavity (Fig. 2.11) was designed in FY 1992 and has been ordered.

The advantage of using superconducting technology for accelerator structures is amplified as the accelerating electric fields and the $\mathrm{rf} Q \mathrm{~s}(\mathrm{Q}=\mathrm{rf}$ stored energy/dissipated energy per cycle) are increased. Carefully controlled acid etching and cleaning of the niobium cells are essential if high fields are to be obtained. At Los Alamos the cells are acid-treated using facilities in MST division. The cells are then transported to AT-1's clean room for processing with ultraclean water. It is essential that ultraclean techniques are followed in the cleaning and handling of the cell. The cavity assemblies are sealed before they are removed from the clean room and subsequently inserted into the liquid helium cryostats (Fig. 2.12).

Vacuum-oven treatment may be required to prevent the so-called "European Q disease." The treatment removes hydrogen from the niobium that can form a hydride during relatively long cool-down times. The presence of the hydride can result in a 
degradation in the $Q$ of the cell. The "disease" has been cured by heal treatment at $80(0)^{\circ} \mathrm{C}$.

If $r f \mathrm{Q}$ values consistently equal to or greater than the design value of $5 \times 10^{\prime \prime}$ are to be obtained, the rf surface resistance of the niobium must be low and the thermal conductivity high. A high thermal conductivity reduces the likelihood of the occurrence of a quench at high electric/magnetic fields. Annealing the niobium in a hightemperature vacuum oven is effective in increasing the achieved values for Qs if the annealing also includes a process that removes oxygen entrapped in the niobium. This is done by titanium gettering in a vacuum oven at $-150\left(0^{\circ} \mathrm{C}\right.$. Smaller 3-GHz cavities can be heattreated with ovens in the structures laboratory. An oven large enough for a complete seven-cell structure is available to us through another division of the Laboratory.

Under certain conditions high-pulsepower (HPP) conditioning of a cell has improved the achievable electric field in a below-par cell. HPP is attractive because it offers an opportunity to recondition an accelerating structure without removing it from the cryostat. The testing reported here was done at
3 GHz. We still need to verify the results all $805 \mathrm{MHz}$.

\section{Achievements}

We developed sufficient datahatse and expertise to allow us 10 advance beyond 3-GHz cavities and concentrate on 80.5 MHz-cavity processing and testing. We have achieved approximalely a factor of $50 \%$ improvement in peak surface field over the previously reported results from the worldwide database at $805 \mathrm{MHz}$ (Fig. 2.13). The average value of the achieved electric field, including an extrapolation to the seven-cell cavity, exceeds the goal for proton acceleration by $\sim 10 \%$.

During the fabrication of the seven-cell structure, niobium half-cells must be ebeam-welded together. However, welding reduces the thermal conductivity at the weld region. The cell is then prone to quenches from the superconducting state quenches that start at the equator weld, a region of relativaly high current density. The fabricator can relieve this problem by heattreating the entire seven-cell structure after welding. However, in our calse, none of the potential fabricators had an oven large enough for the entire sevencell structure. That situation required

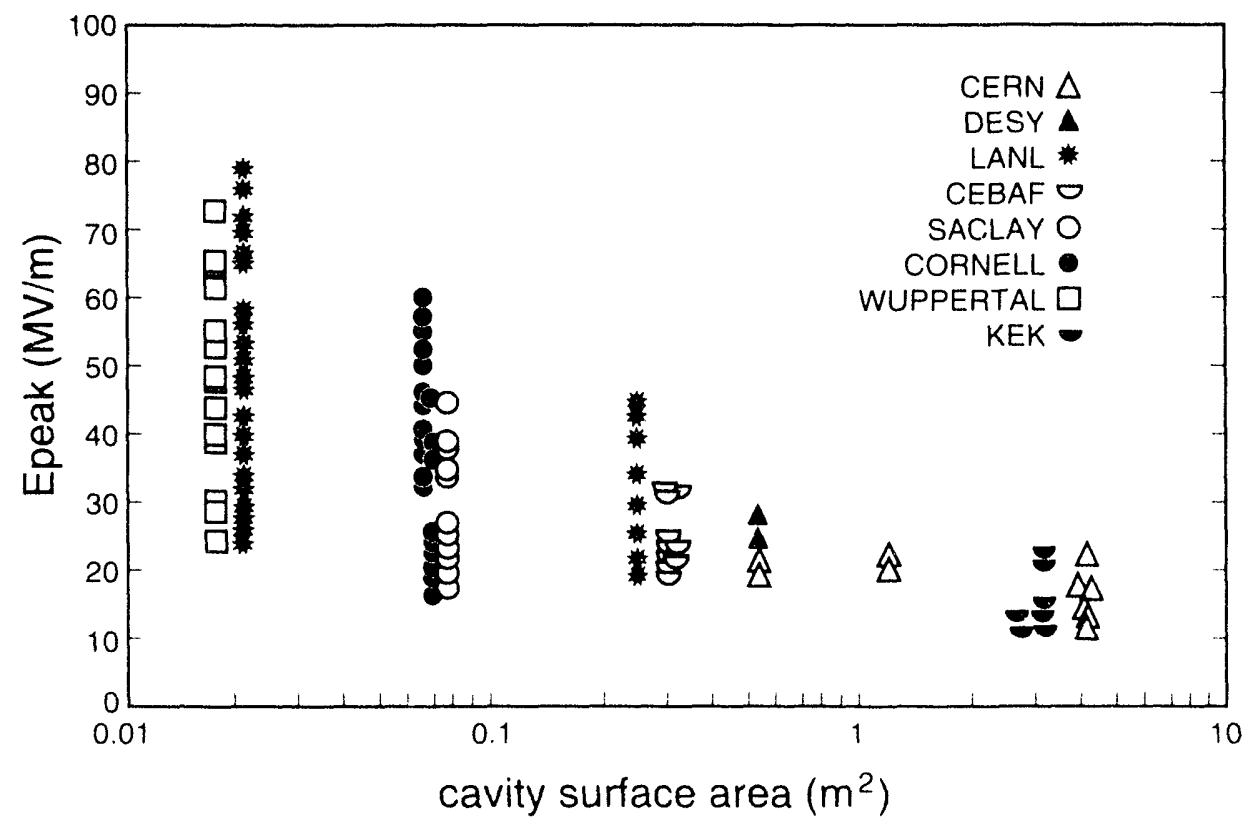

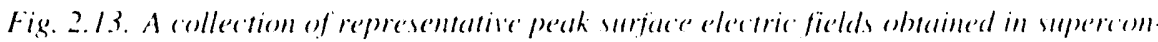

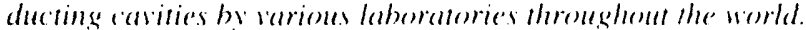

us lo seck a different solution. The iris region of the cell is a region of relattively low-current density: it is, therefore, less sensitive to quenching. We devised a labrication procedure that allowed the fabricator to weld the halfcells at the equator, heall treat the resultant single cell, and assemble the seven-cell structure by welding the individual eells together at the iris. As at lest of this procedure, niobium samples were welded together; some samples were then titanium-gettered heat trealed. Comparison of the residual resistance ratio at $4 \mathrm{~K}$ (RRR) values of the heat-treated and nonheattreated samples showed that the damage to the RRR from e-beam welding could be repaired by the titanium-gettered heat treatment. We successfully tested the procedure using a $3-\mathrm{GH} z$ cell.

This work, combined with additional analyses of structural vibration and mechanical stress, has allowed us to proceed with the next major programmatic step, namely the procurement of a seven-cell structure for tests in the vertical cryostat.

A higher-capacity, roots-type, helium vacuum pump was installed. This pump allowed us to achieve $2^{\circ} \mathrm{K}$ with $8(0.5-\mathrm{MHz}$ cavities for the first time. Also, the time to reach 2 $\mathrm{K}$ with $3-\mathrm{GH}$ / cavities was reduced from $\sim 7$ hours; an entire run can now be completed in a few hours.

A high-pressure $(100()$ psi) ultrapure water rinse wats installed this fiscal year in the structures laboratory s clean room (Fig. 2.14). The water rinse has demonstrated its capability for removing particulates down 100.5 microns in size. This unit is effective in removing dirt from complicated structures such as the welded bellows attachments to the cavity ports, and it was used in the achievement of the record value of 50 $\mathrm{MV} / \mathrm{m}$ for the $80.5-\mathrm{MH} / \mathrm{c}$ cavity. 


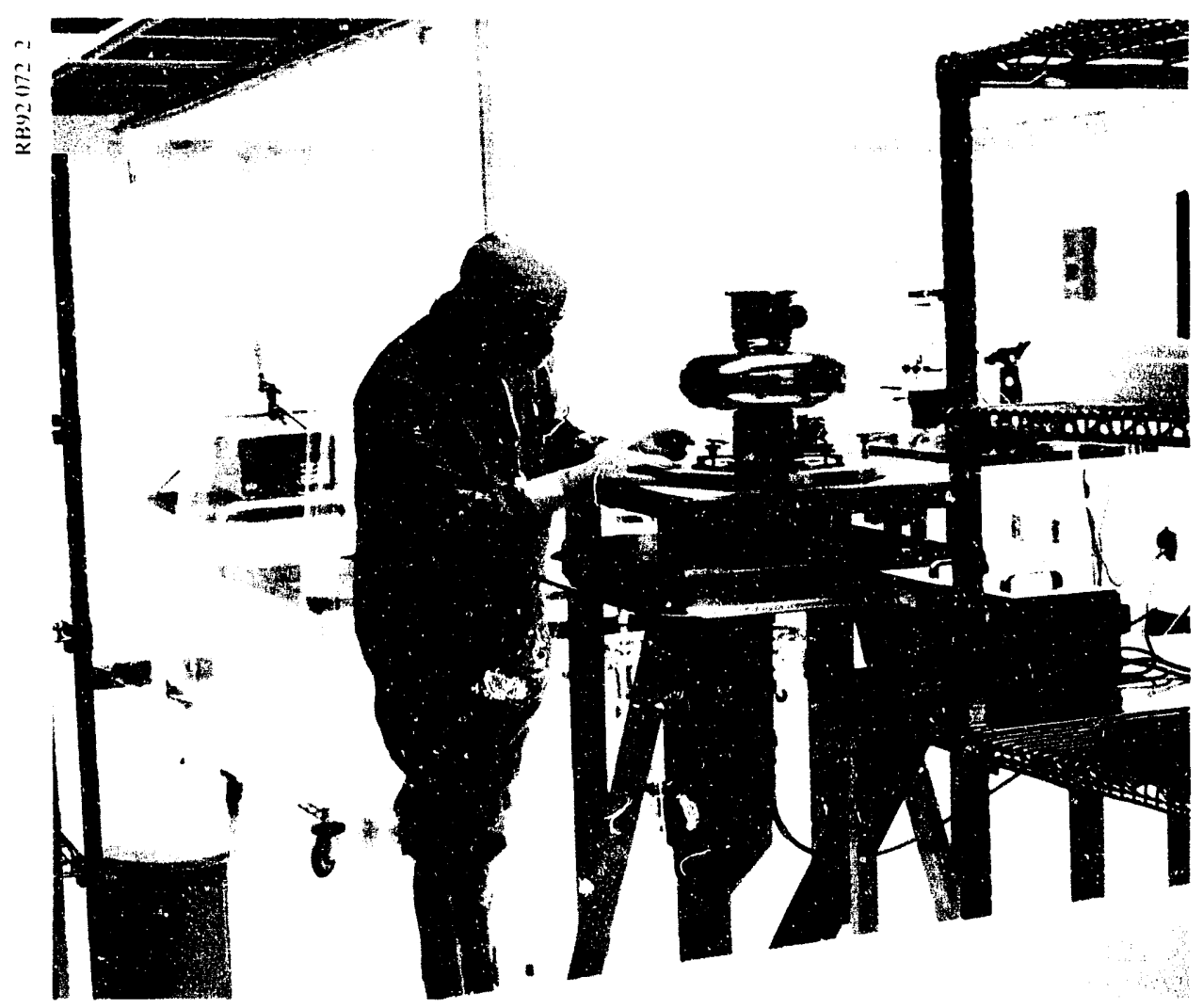

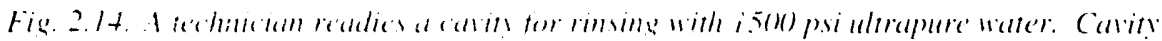

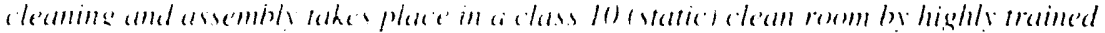

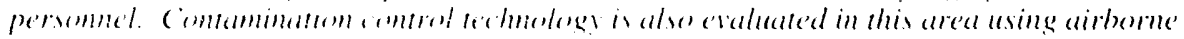

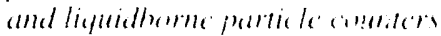

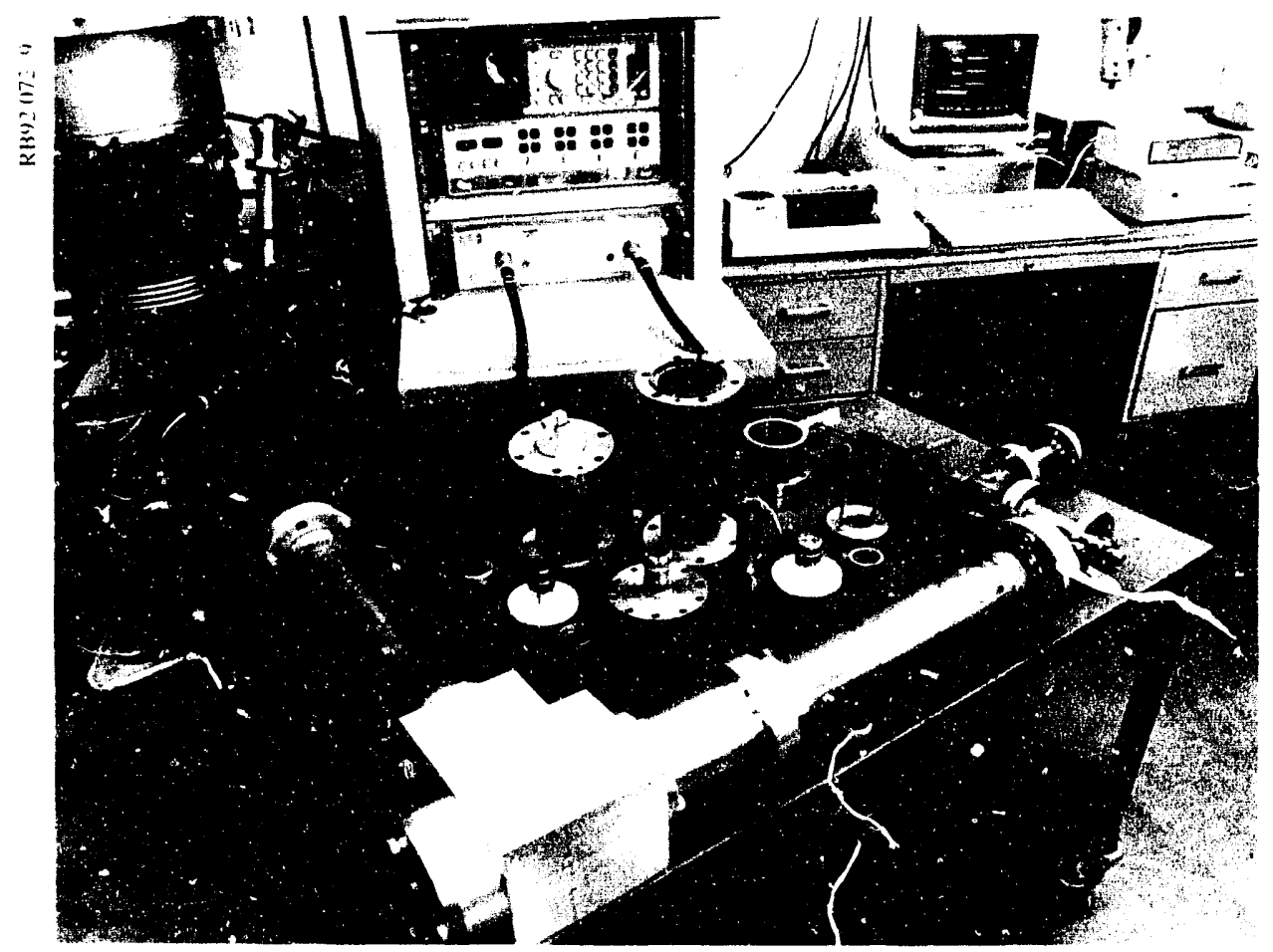

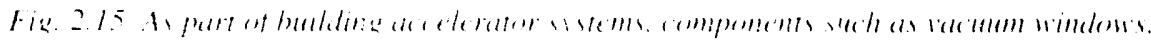

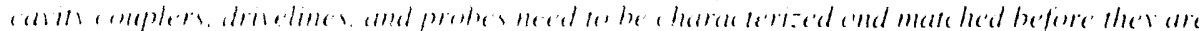

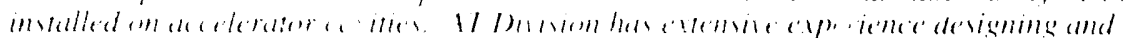

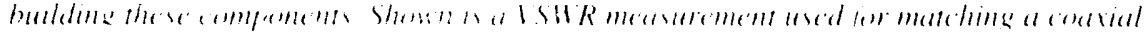

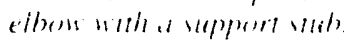

The klystrons in the two COMARK transmitter modules are now operating at $8(0.5 \mathrm{MH}$.. The units previously operated at $500 \mathrm{MHz}$. We now have $10-k W$ cw capability and $10(0)-k W$ pulsed capability at $8(0.5 \mathrm{MHz}$. These units can now be used to high-powerprocess cavities. Programmatic advallages accrue because we have demonstrated that cavities can be reconditioned in situ, thereby allowing us to return a faulty cavity to the line without having to return to room temperature for reconditioning.

Waveguide components (variable coupler, window, and supports) for the high-power test and the seven-cell test have been designed and prototypetested and are now undergoing final fabrication in preparation for the HPP test and the seven-cell cavity test (Fig. 2.15). In addition, the vertical cryostat for the test of the seven-cell structure has been delivered to the Laboratory.

The main program thrust is directed at completing the preparations for and the test of the seven-cell structure in its vertical cryostall. Preparations include digging a pit in the high bay area of MPF-17 for the vertical cryostat. The program will also complete the HPP test of a single cell 805-MH\% cavity. 


\section{Technical Memoranda}

1. J. Billen, "New Root Finder for SUPERFISH on the PC," AT-1:93-301. September 8, 1992.

2. R. Garnet, "Design of the SSC Linac," AT-1:91-409, October 1, 1991.

3. E. Gray, "CEBAF TE Intercavity Coupling," AT-1:92-4(18, October 1, 1991.

4. E. R. Gray, "Cavity to Cavity Coupling Measurement," AT-1:-91:406, October I, 1991.

5. E. R. Gray, "Cavity Models, Cavity Coupling, and Geometric Coupling K," AT-1:91-407, October 1, 1991.

6. E. R. Gray, "Measured Beam Tube Coupling on PIL.AC Calvity Shape," AT-1:91-459, October 6, 1991.

7. E. R. Gray, "Cavity to Cavity Coupling Induced Field Error," AT-1:91-421, October 8, 1991.

8. E. R. Gray, "External Multicell Cavity Field Flattening," AT-1:92-7, January 9, 1992.

9. E. R. Gray, "Stainless Beam Tube Section Power Loss," AT-1:92-18, January 15. 1992.

10. E. R. Gray "805 MH\% RF Cavity High Power Processing," AT-1:92-19, January $16,1992$.

11. E. R. Gray, "Helium Displacement," AT-1:92-180, May 15, 1992.

12. E. R. Gray, "Cavity Frequency Change with Neck Extension," $\wedge$ 'T-1:92-252. August 2, 1992.

13. E. Cray, "A Traveling Wave Cavity Model," AT-1:91-460), Novemher 6, 1991.

14. S. Nath, "Examples of Front-end ATW DTI: s," AT-1:92-128, April 8, 1992.

15. G. Neuschacfer, "Examples 5 Tank 350 MHy APT DTL.," A'T-1:(92-289.

August 28, 1992.

16. G. Neuschatefer. "Experience with DTL, Permanent Magnet Quadrupoles and Recommendations for a Space-Based Version of ("TA." $A \mathrm{~T}-1: 92-131$. April 13, 1992.

17. (i. Neuschaefer, "805 MHz DTL L AMPF Upgrade," AT-1:92-245. August 9. 1992.

18. (3. Neuschaefer. "ATW/APT 175 MHz RI(Q's," AT-1:92-101, March 16, 1992.

19. (i. Neuschacfer, "I75 MHz APT/ATW RI() liximples," AT-1:92-\%).

March 10, 1992.

2(). (i. Spalek. "Summary of Tuning Stresses and l onds for the 7 (ell Developmental Superconducting (avity," $\wedge T-1: 92-11$, April 28. 1992. 
21. G. Spalek, "Power Requirements for 1 Cell and 7 Cell $805 \mathrm{MHz}$ Superconducting Cavities Being Developed at LANL," AT-1:91-410, October 1, 1991.

22. G. Spalek, "Estimate of Overpressure Protection of Developmental 7-Cell Cavity," AT-1:92-29, January 22, 1992.

23. T. Wangler and R. Stevens, "High-Transmission (HT) RFQ Designs for APT/ ATW," AT-1:92-0100, March 11, 1992.

24. T. Wangler, "Accelerating Structures for a Superconducting Proton Linac from a Beam Dynamics Point of View," AT-1:92-0107, March 16, 1992.

25. T. Wangler and L. Young, "RFQ Linear Accelerator for Pet Isotope Production," AT-1:92-324, September 22, 1992.

26. L. Young, "Transient Analysis of PILAC Type Cavities used as Energy Booster on LAMPF," AT-1:92-265, July 29, 1992. 

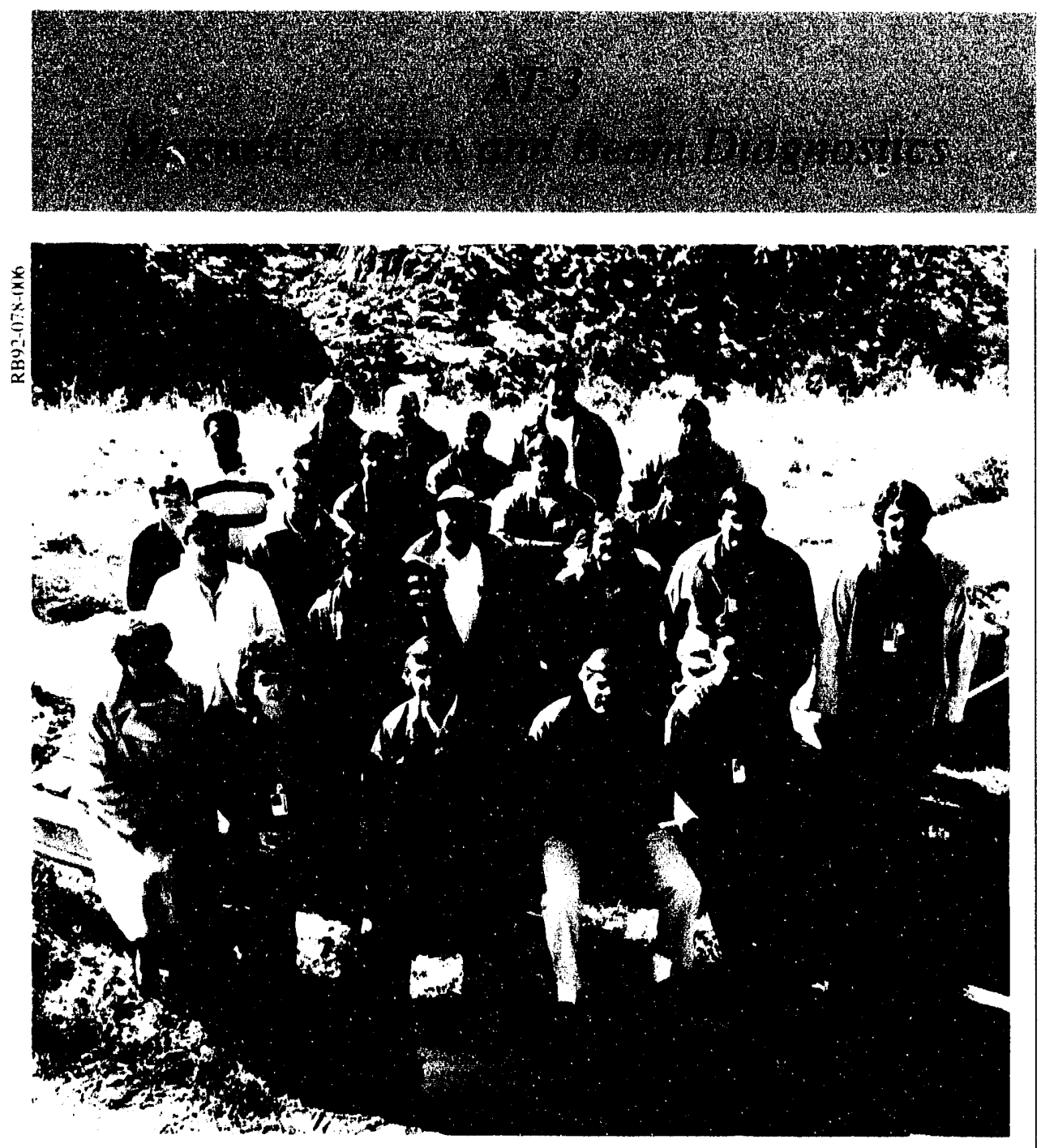

Introduction 39

Backgroumd 39

Achice'eme'nts

Optics Section 39

Becam Diagnesstic Section 4)

Magne' Section 41

Merhanical Design,

Fabrication, and Assembly' Sertiont .42

Future Plans .42

Referencess 42

Terdinical Notes 43

Terdmical Me'moranda 44 


\section{Introduction}

The AT-3 group charter is to "apply beam transport theory, state-of-the-art diagnostic instrumentation, and advanced magnet fabrication and measurement techniques to the design, construction, and commissioning of magnetic optic systems." To fulfill its mission, the group maintains experimental and construction facilities that include a diagnostic laboratory, which fabricates beam-diagnostic electronics; a magnet-measurement laboratory, which performs precision measurements on a wide variety of electromagnets and permanent magnet assemblies; a mechanical design, fabrication, and assembly section; two rooms for laser development, used on the laser induced neutralization diagnostics approach (LINDA) measurement system for the Ground Test Accelerator (GTA); and an area in the high bay, which assembles beamlines. The group is also responsible for managing a staff machine shop located in MPF-18 that houses small lathes, milling machines, grinders, sanders, drill presses, a bead blaster, a bandsaw, a welding and brazing facility, and full vacuum leak checking capabilities.

\section{Background}

Our group employs 33 full-time employees: 12 technicians, 20 staff members, and 1 group secretary (aided by a part-time assistant). In addition, we have one industrial staff partner from Grumman Aerospace Corporation $(\mathrm{GAC})$ and two contract employees.

\section{Achievements}

\section{Optics Section}

In FY 1992 the Optics Section continued to develop those capabilities necessary to study and design beamlines and output optics systems that must meet very demanding requirements. We also created designs and conducted studies that support a variety of Los Alamos projects.

\section{New Techniques and}

\section{Code Development}

In a collaborative effort with J. van Zeits at the University of Maryland, we developed computer codes to calculate higher order aberrations, now making it possible to fully optimize high-order aberration coefficients. These results have been checked against numerical integration and other codes developed at GAC that are not capable of optimization. Consequently, a significant development, the concept of contouredfield telescopes, was developed. The principle is that the longitudinal dependence of each multipole is determined by the requirement that, while constrained to serve its primary function, it also does the least damage to the next higher order of aberration. This technique generates designs in which the residual high-order aberrations are reduced by one or two orders of magnitude. Consequently, we have been able to apply this work to the GTA and the neutral particle beam space experiment (NPBSE).

We have studied various first-order achromatic $180^{\circ}$ bend designs, including space-charge effects. We developed the ACHRO code to help us quickly generate and evaluate designs for new requirements. This work has been applied to the GTA Optics Experiments design.

We refined our nonlinear beam redistribution method and created designs for several projects described below. Our designs take the beam leaving an accelerator and generate a distribution that is, to a good approximation, uniform over a large rectangular region on the target.

We verified some aspects of Parmila emittance growth predictions caused by quadrupole roll errors in linacs. In this work, we used P. Channell's mismatchemittance-growth theory to estimate emittance growth in terms of the rollangle error.

We continued to develop the TRACE3D code, which is used frequently for the first-order beamline design. We ported the code to UNICOS, replacing the previous CTSS version for the Cray. We also completed an implementation for SUN workstations, in which we wrote a replacement for the PLOTI () library, eliminating the need for a Tektronix terminal or emulator. This implementation uses the same TRACE3D code used on VAX VMS systems, eliminating the need to maintain separate SUN and $\mathrm{VAX}$ versions.

We continued to develop the BEDLAM code, an experimental three-dimensional, high-order space-charge code that represents a beam by the moments of its phase-space distribution. We tested the space-charge algorithm and verified that the moment approach, as expected, works very well in generating beams that are matched to high order in the presence of nonlinear forces.

\section{Beamline Designs and Project Support}

We completed a new design of the GTA high-energy beam transport (HEBT) that includes an insertable beam dump. Variable field permanent magnet quadrupoles (PMQs) expand the beam in the beam-dump mode.

We designed a $3.1-\mathrm{m}$ bend for GTA, which consists of three five-cell, firstorder achromats. This design was studied extensively to determine effects of various magnet and beam errors and to determine the aperture and steering requirements.

We determined that an energy-corrector cavity is required for GTA only if the energy errors are beyond a certain value, that a bunch rotator is not needed, and that we need a (wofrequency (425 and $850 \mathrm{MH} /$ ) momentum compaction system at the end of the bend. The system requirements were determined using a newly developed specialized code that computed the fraction of beam on a distant target. using an actual telescope design to estimate chromatic effects. 
The measured beam behavior in the intermediate matching section (IMS) of GTA was found to be in good agreement with our simulations; however. the existing beamline design eould not provide a perfect match into the linac. The matching range of the IMS wats insulficient to handle the somewhat low brightness of the present beam. A redesign was proposed in which the PMQ steerer magnets would be rehored to lower their gradients. The proposed design is capable of matching a much wider range of beams exiting the radiofrequency quadrupole (RFQ).

We created a specialized version of the ETRACEM code for the dual-axis radiographic hydrodynamic test (DARHT) project, adding leatures such as the ability to account for random errors in the magnets.

We designed a beam-redistribution system that produces uniform or hollow distributions at the target for the Energy Selective Neutron Itradiation Test (ESNIT) facility. We determined losises and effects of duodecapole components in the octupole magnets.

For the Accelerator Production of Tritium (APT) project, we designed a beam-redistribution system that produces a large uniform beam at the larget and characterized the nominal heam-intensity distributions and their operational variabilities resulting from beam jitler, beam-energy shifts following rf-module failure, and beamemitlance shifts following acceleralorquadrupole failures. Because the specifications include multiple targets, the HEBT designs used rl deflectors.

For the Ios Alamos Neutron Scallering Center II (IANSCL II) project, we identilied and studied a candidate ring design and dimensioned its apertures (o) accept 1/3 of the beam required at 800 MCV and $5 \mathrm{MW}$. Additionally, we studied the ring design's injection chicante hump-magnet scheme, and extraction section. We also identified a possible 2-(ic) ring design.
In FY 1902 we designed optics and conducted studies for the Acceleraltor Transmutation of Waste, pion linac, International Fusion Matcrials Irradiation Facility, NPBSE, (EED), and the Luggage Detection System.

\section{Beam Diagnostics Section}

The AT-3 Beam Diagnostics Team was involved with several programs in FY 1992. The majority of our efforts were for the GTA program, but we also provided diagnostics support for the Advanced Free-Electron Laser (AFIEL), Average Power Laser Experiment (APLE), APLE Prototype Experiment (APEX) Grumman Injector, and the Supereonductor Super Collider programs.

The heam diagnostics team provided GTA with permanent and characterizalion baam diagnostics for several heam experiments, including tests for the IMS, which matches the heam from the RFQ to the input of the drilt tube linac (DTL.). The permanent beam diagnostics included within the IMS were three microstrip probes, a beam current monitor, and a video beam profile. A diagnostics plattorm or plate, for beam characterization hats a transverse emiltance measurement (typically described as a slit and collector emit(ance scamner), a beam current monitor. three more microstrip probes, al phasespread meatsurement, and a longitudinal emillance measurement using a laser induced neutralization diagnostics approach (LINI)A). Several papers have heen written describing these various meatsurements and the dala acquired from these systems and were published at the I.INAC and PAC conferences this past year. Additionally, our team has wrillen many $\mathrm{A} \mathrm{T}-3$ lechnical notes and several meatsurement operators manuals.

( )ne beam diagnostics system completely designed, prototype, and beam lested in limiled yuantities was the beam-loss moritor syslem, which comsists of an ion chamber and lexal preamplifier whose signal is cabled lo another processing electronics module. The complete beam-loss monitor system provides $(w 0$ fianctions for the (iTA. The primary function is to provide a fast $(<10$ ms) response signal if a catastrophic accident should occur in which the accelerator and beamline components anc placed in peril because of direcl beam impingement. For particle beam energies above 5 to $8 \mathrm{MeV}$, this interaction would produce lower energy gammas, which would be sensed by the system?s ionization chamber. If the gamma dose late is sufficiently high, a signal is sent to a fast protect system that, in turn, shuts down the beam source. The second function of the beam-loss monitor system is to provide smaller raltes of heam loss, which in turn can give the GTA commissioners more information about the beamline and accelerator.

AT-3 also contributed to various electron beamlines allached to the freeelectron laser (FEL). Both the AFEL and APFX fatcilities have received beam position measurement systems that use capacitive probes and processing electronics similar to those used for the G'TA. Additionally, we began work on the API.F project, an electron linac/ beamline for high-average power FEL.s all Bocing in Seattle, hy performing heam lests on a flying-wire style of charged-beam profile mealsurements (FWBPM).

We modified the design of the prototype FWBPM system, originally developed fir use on the (iTA, Io improve its perfomantece and to aldapt it (o) measure beam-profiles for the APL.t:/HP() high-current electron accelerator. The signall's amplitude. measured either directly off the wire, or indirectly from detectors locilled near the wire, is proportional to the beam that intercepts the wire. Hence measuring the signal as the wire moves through the heam will map out the beam profile along the axis perpendicular to the wirces molion. The APl l:/ HP( ) liWBPM syslem has a pair of 3.5 . dia. micron carbon wires mounted al 
right angles to one another on a $17-\mathrm{cm}$ diameter wheel. The two orthogonal wires allow both the $X$ and $Y$ beam profiles to be measured in the same scan. A servo motor is used to accelerate the wheel from rest to its peak speed in less than half a revolution, at which point the two wires intercept the beam and the wheel is decelerated back to rest before completing one full revolution. The electron beam that intercepts the wire produces secondary electrons that are detected either by measuring the charge depletion current on the wires or by collecting the secondary electrons on a pair of positively biased collectors mounted up and down stream of the wires. The goal of this system is to measure the profile of a $\sim 1 \mathrm{~ms}-$ long, $\sim 1 \mathrm{~mm}$ diameter high-current electron beam with a resolution of $100 \mathrm{~mm}$ or better without over heating and breaking the fine carbon wire. For the wires to survive in the APLE/HPO beam, the wire must be capable of sweeping through the beall at a speed of $20 \mathrm{~m} / \mathrm{s}$ or better. Achieving these speeds in just half a revolution does not leave enough time for the wheel velocity to stabilize before the wires sweep through the beam. To overcome this problem, the wheel velocity is recorded as the wires move through the beam, allowing the data to be corrected for any variations in velocity.

The APLE/HPO FWBPM system has been tested at the APEX electron accelerator using a high-intensity lowduty beam of $\sim 40$-MeV electrons. However the typical macropulse length of the APEX beam is too short $(<20$ $\mathrm{ms})$ for the moving wires to intercept. Instead the wires were moved slowly through the beam to map out a beam profile averaged over many macropulses. Preliminary results indicate that the signal strength measured from the wires and charge collectors agrees with the calculated signal strength: and a preliminary beam profile measurement also indicates that the profile measured with this system had roughly the same shape and width as the profile observed on a nearby video screen. We will conduct future studies to refine this measurement, to determine the measurement resolution, and to determine which method of signal detection yields the best results. Preliminary results indicate that the signal-to-noise ratio is considerably better on the charge collection ring, which if accurate would greatly simplify the mechanism by eliminating the need to make electrical contact with the wires mounted on the rapidly moving wheel.

Finally, we delivered several toroids (beamline devices used to measure beam current) and a microstrip measurement system to GAC. We have extended this contract to include two more microstrip systems which will be used with a small test stand for experiments with injector automation experiments.

\section{Magnet Section}

\section{Magnet Measurement}

In July 1992, we completed our final cryo mapping of the permanent magnet DTL quads for the GTA. Over 200 cryo maps were taken to measure the gradient length (GL) product vs temperature ( $T$ ) dependence of the 140 PMQs need for the DTL. This effort occurred over a year and a half as the magnets were received from the vendor. The cryo mapper specifically designed for this task worked remarkably well; once operational, it experienced very little down time. It measured the GL vs T of the cryo quads from room temperature to $20 \mathrm{~K}$ and back to room temperature with an absolute uncertainty of $\pm 0.5 \%$ in a cycle time of approximately 3 hours per magnet.

A Rotating Sample Magnetic Moment Mapper (RSM3) was built by the AT -3 magnet lab to measure the direction and magnitude of the magnetic moment of permanent magnet material samples. The design for the RSM 3 is patterned after a similar device built by Lawrence Berkeley Laboratory. ${ }^{1,2}$ It consists of a pair of $45-\mathrm{cm}$ diameter coils each wound with 850 turns of \#30 copper wire. The coils are placed in a Helmholtz configuration, and the sample to be measured is rotated at the center of the two coils by a small direct current (dc) motor. A 1000 -pulse per revolution incremental encoder is connected to the dc motor, and the coils' voltage is integrated and read in every 20 encoder pulses by a Metrolab digital integrator. Data acquisition and control are through a GPIB interface to a Macintosh Ilci running Labview. Magnet segments are mounted in a cubical holder that orients the sample's $x, y$, or $z$ axes parallel to the axis of rotation. The amplitude and phase of the signal, measured as the sample is rotated on its three axes, determines the magnetic moment in the plane perpendicular to the axis of rotation. These results can then be used to calculate the $x, y$, and $z$ components of the magnetic moment, the overall magnitude (M), and angular deviation of $M$ from the desired orientation (DQ). The uncertainty in the absolute measurement of $\mathrm{M}$ is estimated to be $\pm 1 \%$, and the uncertainty of determining the absolute DQ is estimated to be $\pm 0.5^{\circ}$. The relative uncertainties of $M$ and $D Q$ are much better, $\pm 0.05 \%$ and $\pm 0.25^{\circ}$, respectively.

\section{Magnet Design and Fabrication}

Four variable field permanent magnet quadrupole (VFPMQ) doublets and one singlet were designed and fabricated by AT-3 for use on the AFEL. The basic design of the AFEL VFPMQ is similar to the magnets built for the GTA IMS, which are patterned after the conceptual design proposed by K. Halbach." The magnetic design criteria for the magnets were a clear bore diancter of $26.2 \mathrm{~mm}$, a quadrupole gradient that can be continuously varied from () to 60 $\mathrm{T} / \mathrm{m}$, minimal allowed harmonics, and 35-mm-long pole tips. Two key mechanical design features of these magnets were that the doublets were made by mounting two VFPMQs $1 \mathrm{~cm}$ apart in the same housing and that the polarity of each VFPMQ could be easily reversed without disassembling the unit. The mechanical design wats 
found to work well and the magnetic measurements were in excellent agreement with the calculated results. The magnets were installed on the AFEL and have been in continual use since June 1992.

Additional subroutines for threedimensional modeling of large-bore optics elements were incorporated in MARYLIE and TLIE, the two highorder optics codes being developed in collaboration with the University of Maryland. These new subroutines allow us to compute error effects, including "hidden" multipoles in permanent magnet quadrupoles, i.e., field components arising from axial components of magnetization. These field components integrate to zero and are not seen in integral field measurements, but can affect higher-order optics.

A preliminary design for a prototype ferrite kicker magnet module for use in proton storage rings was completed in FY 1992. The design incorporates various features to minimize the rise time, including use of a parallel speedup capacitor and a series saturable inductor.

The cylindrical current-sheet magnet concept was extended to short iron-free quadrupoles. These magnets are designed to produce a magnetic field with nearly perfect $\sin 2 f$ symmetry about the beam axis; the residual integral error multipoles due to finiteturn-number effects are zeroed out by small shifts in the conductor positions specified by a computer algorithm developed in 1992. Precise conductor placement is achieved by fixing the superconducting wires in grooves that are machined in a particular pattern in a cylindrical surface by a numerically controlled milling machine.

\section{Mechanical Design, Fabrication, and} Assembly Section

The mechanical design, fabrication and assembly section provides design. quality control of design packages, liaison work, fabrication, assembly, installation. machining, welding, and vacuum leak certification. This service supports other sections within AT-3, AT Division, other accelerator laboratories. Grumman Aerospace Corporation, the U. S. Navy, Boeing Space and Science Division and the GTA program.

Some of the components that this section has provided are: beamline diagnostics, full sections of beamlines, experimental beamlines, beamline support structures, resistive dipole and quadrupole magnets, harps, vacuum boxes, steering magnets, Lambertsontype correction dipoles and quadrupole magnets, variable field permanent magnet quadrupoles, and variable field permanent magnet dipoles.

\section{Future Plans}

Our future plans are to complete the beam diagnostics for the GTA accelerator and begin the beam diagnostic work for the GTA bend and telescope. We will continue to build beam diagnostics for APLE, and increase our diagnostics support for SSC.

We will increase our magnet design, building, and mapping support for the SSC. We also anticipate building magnet mapping devices for the SSC.

The AT-3 optics section will support the bend and telescope design for GTA, support storage ring design for LANSCE II, and support the ABC projects with optics design for creating uniform beam illumination target design, and increase our support for the SSC.

\section{References}

1. D. Nelson, P. Barale, M. Green, and D. Van Dyke, IEEE Transactions on Magnetics, 24, No. 2. 1098 (1988).

2. D. Nelson, P. Barale, M. Green, and D. Van Dyke, 9th International Conference on Magnet Technology, p 735 .

3. K. Halbach, Nuclear Instruments and Methods 206, 353-354 (1983). 


\section{Technical Notes}

The following AT-3 Technical Notes were written during the report period.

1. R. E. Shafer, "Effect of Alignment Errors in the GTA-24 180 Degree Bend," Los Alamos National Laboratory Technical Note AT-3:91-16, October 1991.

2. B. Blind, "Beamline Configuration for Simulation Delivery of $p+$ and $p$ - to PILAC," Los Alamos National Laboratory Technical Note AT-3:91-17, October 1991.

3. B. Blind, "A Beam Delivery System for the ATWE," Los Alamos National Laboratory Technical Note AT-3:91-18, October 1991.

4. R. Connolly, "The Optics Design for a Spectrometer to Measure the Energy Distribution of 10-100-MeV Proton Beams," Los Alamos National Laboratory Technical Note AT-3:91-19, November 1991.

5. C. R. Rose and D. Wells, "Owner's Manual, 'Position Detector'," Los Alamos National Laboratory Technical Note AT-3:91-20, November 1991.

6. P. L. Walstrom, "Magnetic Fields from Distribution of Dipoles on Cylindrical Surfaces," Los Alamos National Laboratory Technical Note AT-3:91-21. November 1991.

7. B. Blind, "A Choice of Point-to-Parallel Focusing Modules," Los Alamos National Laboratory Technical Note AT-3:92-1, February 1992.

8. R. H. Kraus, Jr., "Permanent-Magnet Material Applications in Particle Accelerators," Los Alamos National Laboratory Technical Note AT-3:92-2, February 1992.

9. J. D. Gilpatrick, "Microstrip Measurement Algorithms," Los Alamos National Laboratory Technical Note AT-3:92-3, February 1992.

10. P. L. Walstrom, "Temperature Rise due to Gorter-Mellink Mutual Friction in the Pilac Horizontal Cryostat," Los Alamos National Laboratory Technical Note AT-3:92-5, March 1992.

11. B. Blind, "Beam-Redistribution System for ESNIT," Los Alamos National Laboratory Technical Note AT-3:92-6, May 1992.

12. B. Blind, "Useful Formulas for Cylindrical Resonators in TM110 Deflecting Mode," Los Alamos National Laboratory Technical Note AT-3:92-7, July 1992.

13. J. D. Gilpatrick, "Double Balance Mixer Operation used as Phase Synchronous Detector as Applied to Beam Position and Intensity Measure," Los Alamos National Laboratory Technical Note AT-3:92-8, July 1992.

14. C. R. Rose, "Operator's Guide 425/850 MHz Down Converter Version 3.0," Los Alamos National Laboratory Technical Note AT-3:92-9. September 1992. 


\section{Technical Memoranda}

The following AT -3 Technical Memos were written during the report period.

1. E. A. Wadlinger and W. P. Lysenko, "Maximum Energy Jitter from the 24-MeV GTA Linac," AT-3:91-573, October 3, 1991.

2. W. P. Lysenko, "TRACE3D on Sun Workstations," AT-3:91-599, October 15, 1991 .

3. W. P. Lysenko, D. P. Rusthoi, and E. A. Wadlinger, "GTA Bend Aperture Requirements," AT-3:91-601, October 16, 1991.

4. W. P. Lysenko, "Experiment 3 Physics Design Status," AT-3:91-605, October 16, 1991 .

5. W. P. Lysenko, "Status of Linac Quadrupole-Roll-Error Study," AT-1:91-620, October 22, 1991 .

6. W. P. Lysenko, "Update on TRACE3D for Sun Workstations," AT-3:91-665, November 19.1991.

7. E. A. Wadlinger, D. P. Rusthoi, and W. P. Lysenko, "GTA Bend Aperture Requirements for the 3.1-m Bend Experiment," AT-3:92-41, January 24, 1992.

8. W. P. Lysenko, "HEBT for 3.1-m Bend Experiment," AT-3:92-145, March 17, 1992.

9. W. P. Lysenko, "HEBT Steering and Matching Range," AT-3:92-386, August 11.1992 .

10. D. P. Rusthoi, “3.9-m Bend (Lattice \#12): Error Studies with Acceptance Beam." AT-3:91-588, October 8. 1991.

11. D. P. Rusthoi, "3.9-m Bend (Lattice \#12): Quadrupole Field Error Studies," AT-3:91-597, October 11, 1991.

12. D. P. Rusthoi, "GTA Bend Aperture Requirements," AT-3:91-60)1, October 16.1991.

13. D. P. Rusthoi, “3.9-m Bend (Lattice \#12): R-Matrix Elements for Steering Studies," AT-3:91-6.31, Octoher 28, 1991.

14. D. P. Rusthoi, "Study of Coherent Achromats with Space Charge," AT-3:91-644. November 5. 1991.

15. D. P. Rusthoi, "3.9-m Bend (Lattice \#12): Stecring Algorith Application," AT-3:91-681. November 27, 1991.

16. D. P. Rusthoi, “3.1-m Bend (Lattice \#1.3): New Lattice Parameters," AT-3:91-7()8. December 16. 1991.

17. D. P. Rusthoi, "3.1-m Bend (Lattice \#1.3): Second Order Analysis," AT-3:91-721, December 23, 1991. 
18. D. P. Rusthoi, "3.1-m Bend (Lattice \#13): Quadrupole Field Error Study," AT3:92-003, January 6, 1992.

19. D. P. Rusthoi, "3.1-m Bend (Lattice \#13): Space Charge Study," AT-3:92-017, January 13, 1992.

20. D. P. Rusthoi, "3.1-m Bend (Lattice \#13): Magnet Skew Study," AT-3:92-022, January 15, 1992.

21. D. P. Rusthoi, "3.1-m Bend (Lattice \#13): Error Studies with Acceptance Beam," AT-3:92-024, January 16, 1992.

22. D. P. Rusthoi, "3.1-m Bend (Lattice \#13): Dispersion \& R-Matrix Data," AT-3:92-032, January 22, 1992.

23. D. P. Rusthoi, "3.1-m Bend (Lattice \#13): Steering Error Calculations," AT-3:92-040, January 24, 1992.

24. D. P. Rusthoi, "GTA Bend Aperture Requirement for the 3.1-m Bend," AT-3:92-041, January 24, 1992.

25. D. P. Rusthoi, "3.1-m Bend (Lattice \#13): Steering Algorith Application," AT-3:92-059, February 3, 1992.

26. D. P. Rusthoi, "GTA IMS: Emittance Measurements/Predictions Compared," AT-3:92-113, February 27, 1992.

27. D. P. Rusthoi, "GTA IMS: Emittance Measurements/Predictions Compared \& Model Corrected," AT-3:92-123, March 4, 1992.

28. D. P. Rusthoi, "3.1-m Bend (Lattice \#13): Effect of Higher Dipole Tolerances ...," AT-3:92-138, March 13, 1992.

29. D. P. Rusthoi, "GTA IMS: VFQ Settings for Rounder Beams at ES5," AT-3:92-179, April 14, 1992.

30. D. P. Rusthoi, "DARHT: Match and FODO Lattice," AT-3:92-214, May 12, 1992. 

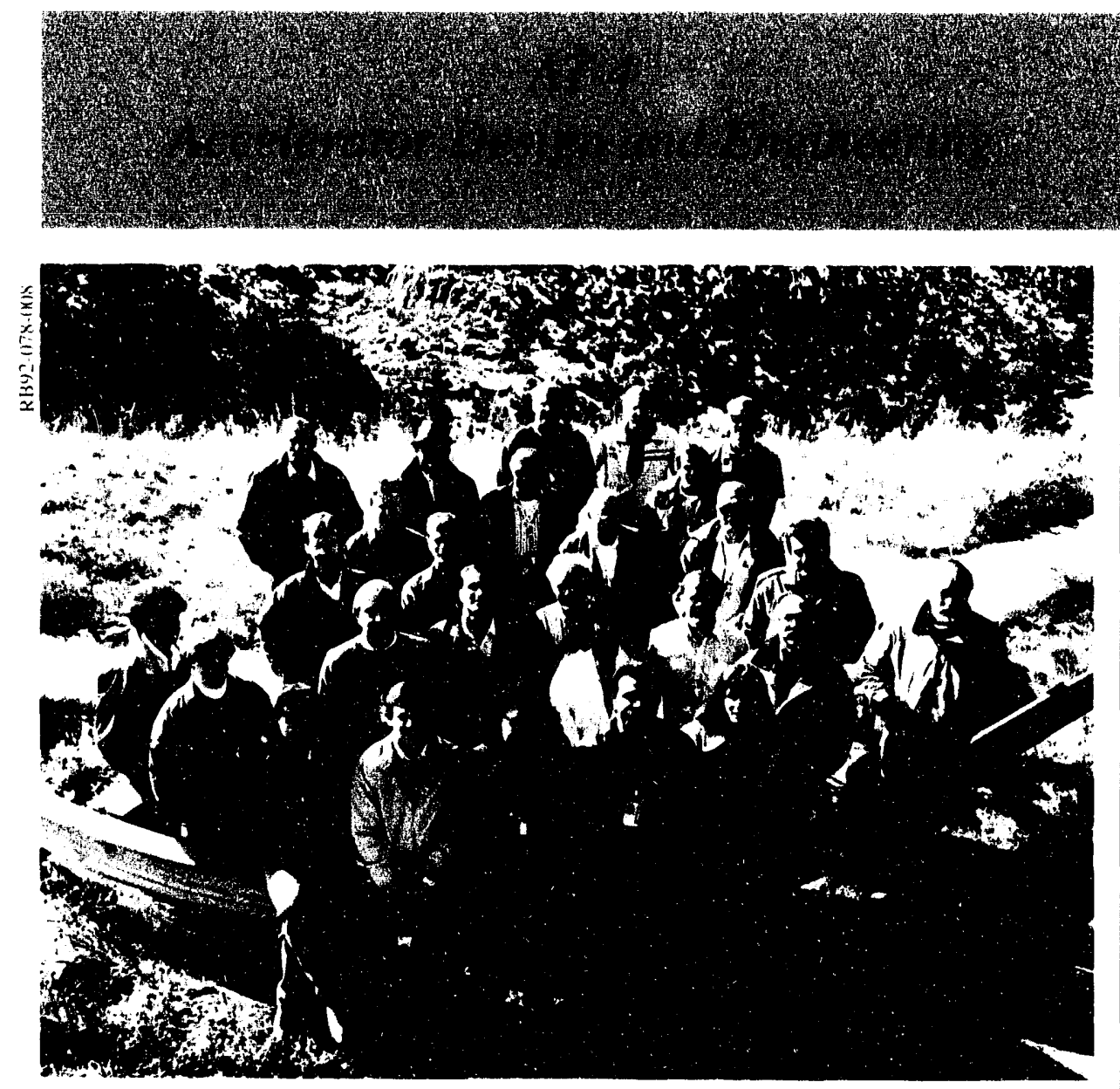

Introduction 47

Achievements. 48 GTA 48

Design of the Accelerator for Production of Tritium (APT) and Transmutation of Waste (ATW)

Seven-Cell Superconducting Carity' and Superconducting Cavity Chains

Support of Others .57

Environment, Safety, and Health $(E S \& H)$

References .59

Technical Memoranda. 59 


\section{Introduction}

AT-4 represents one of the strongest mechanical engineering organizations at the Los Alamos National Laboratory (LANL), combining the individual strengths found in other Laboratory mechanical engineering organizations and focusing them on the design of radio-frequency ( $r f$ ) accelerators. The group is currently composed of five sections: analysis, mechanical engineering, mechanical drafting, test engineering, and drawing production and quality assurance (QA). The centralization of these sections has allowed us to build a stronger capability in AT Division.

The engineering analysis section has developed and supported a sophisticated analysis capability incorporating PATRAN/ABACUS software and Silicon Graphics engineering work stations. This system allows us to model both the structural as well as the thermal response of very complex geometries as well as the vibrational response of systems. The analysis section is also responsible for developing computer aided engineering ( $\mathrm{CAE}$ ) techniques for accelerator development. We are currently using these techniques to build cold models of complex of structures using a stereo-lithography technique. The basic goal of the project is to build an actual cold model of the part in a few hours based on an electronic model of the part (computer aided design [CAD] solids representation) using a stereo-lithography technique combined with a copper deposition process. This electronic model would also be coupled with our thermal and structural analysis software to allow us to analyze the part from a common data base.

Our mechanical engineering section is responsible for the mechanical design of accelerator components, including engineering accelerator cavities, magnets, couplers, diagnostics and beam line components such as vacuum vessels, support structures, and cooling systems. This section is also respon- sible for designing cryogenic accelerator components as well as for developing and implementing the engineering methodology necessary to the success of our projects. Methodology includes physics and engineering criteria definition documents, a design review process, value engineering, and training new engineers.

Our mechanical drafting section produces machine drawings that vary in scope from sketches to manufacturing drawings. Manufacturing drawings are produced using geometric tolerancing to ANSI Y14.5 standards and incorporate drawing system requirements based on DoD-D-1000 standards. The drafting section incorporates a formal drawing checking procedure as well as a formalized drawing change procedure. This section uses a CAD system with SiG stand-alone work stations, which operate in a secure environment, and ICEMDDN CAD software. In addition, the mechanical drafting section manages the electronic archival of drawings.

The test engineering section is responsible for assembling and testing hardware designed by the engineering and design sections; designing assembly and testing fixtures; and assembling complete accelerator cavities including tuners, magnets, positioning devices, $\mathrm{rf}$ couplers, diagnostics, and cooling manifolds. Typical testing involves pressure and flow testing of accelerator cavities, vacuum and cryogenic testing of components, load, positioning, and repeatability testing of magnet positioners, and testing of rf tuners. This section operates three cryogenic test facilities capable of operating at $20 \mathrm{~K}$ with refrigeration capacities up to $750 \mathrm{~W}$ and operates a vacuum laboratory that incorporates ultra-high vacuum test apparatus.

Our drawing archival and QA section is responsible for drawing production, reproduction, hard copy archival, and QA functions in the group. This section also operates the drawing security vault and maintains the security re- quirements for CAD systems as well as for drawings. Responsibilities also include meeting the Department of Energy (DOE) program requirement for three levels for drawing archival as well as for adhering to a drawing system. In addition, DOE programs are beginning to apply QA procedures to the design process by requiring design reviews, design notes, and specifications documentation. Consequently, this section is responsible for implementing these QA functions for the other sections in the group as required.

In FY 1992, we have witnessed several successes in project related activities within the group. The Ground Test Accelerator (GTA) has been commissioned up to and including the first drift tube linac (DTL) module. This accelerator, engineered and constructed by AT-4, has been immensely successful; both the RFQ and the DTL have operated flawlessly. The intertank matching section (IMS), which required the $\mathrm{rf}$ couplers be redesigned and the steering magnets be modified to account for a broader operating requirement, has also t. zen a success, particularly because it is such a highly complex piece of hardware. AT-4 has also successfully progressed toward a preliminary design of the GTA optics system and is beginning the conceptual engineering design of an upgrade to the Proton Storage Ring facility, which could represent a major engineering project in the next few years. The group has continued to provide engineering support to other groups and divisions associated with accelerator work. We participated in the superconducting RFQ design, an accelerator for the Federal Aviation Administration for baggage inspections, and supported the beamline components design for the Clinton $\mathrm{P}$. Anderson Meson Physics Facility (LAMPF) accelerator. We are a maturing organization who has developed the key elements to support a successful engineering organization. 


\section{Achievements}

\section{GTA}

\section{DTL Assembly and Testing}

The drifi lube linac (DTL) comprises the major portion of the 24-MeV GiTA. It consists of 10, 1-meter long, 8.50 $\mathrm{MHz}$ cavities containing a total of 1.30 drift-lubes. It resides immediately downstream of the Intertank Matching Sections (IMS) on the GTA beamline.

Four DTL modules have been completed through high power ri acceplance testing at operating temperature $(20 \mathrm{~K})$. One additional module is completely assembled and in the process of testing. The remaining five modules are in various stages of assembly.
All components are available exeept the remaining drift-lubes for modules \#8-

10. which are being completed al Westinghouse Hanford Company. During FY 199.3. final assembly and lesting of the remaining DTL, modules is planned for eventual installation on the GTA beamline.

Acceptance testing of each module is an extensive set of tests consisting of (1) room temperature alignment of the drifttubes. (2) room lemperature resonant frequency tuning of the cavity, (3) flow orificing and pressure/leak lesting, (4) low power eryogenic testing to evaluate changes in drift-tube alignment and resonant frequency from room lemperalure, and (5) high-power if conditioning of the cavity prior $(0)$ installation on the beamline. The D'TL modules appear 10

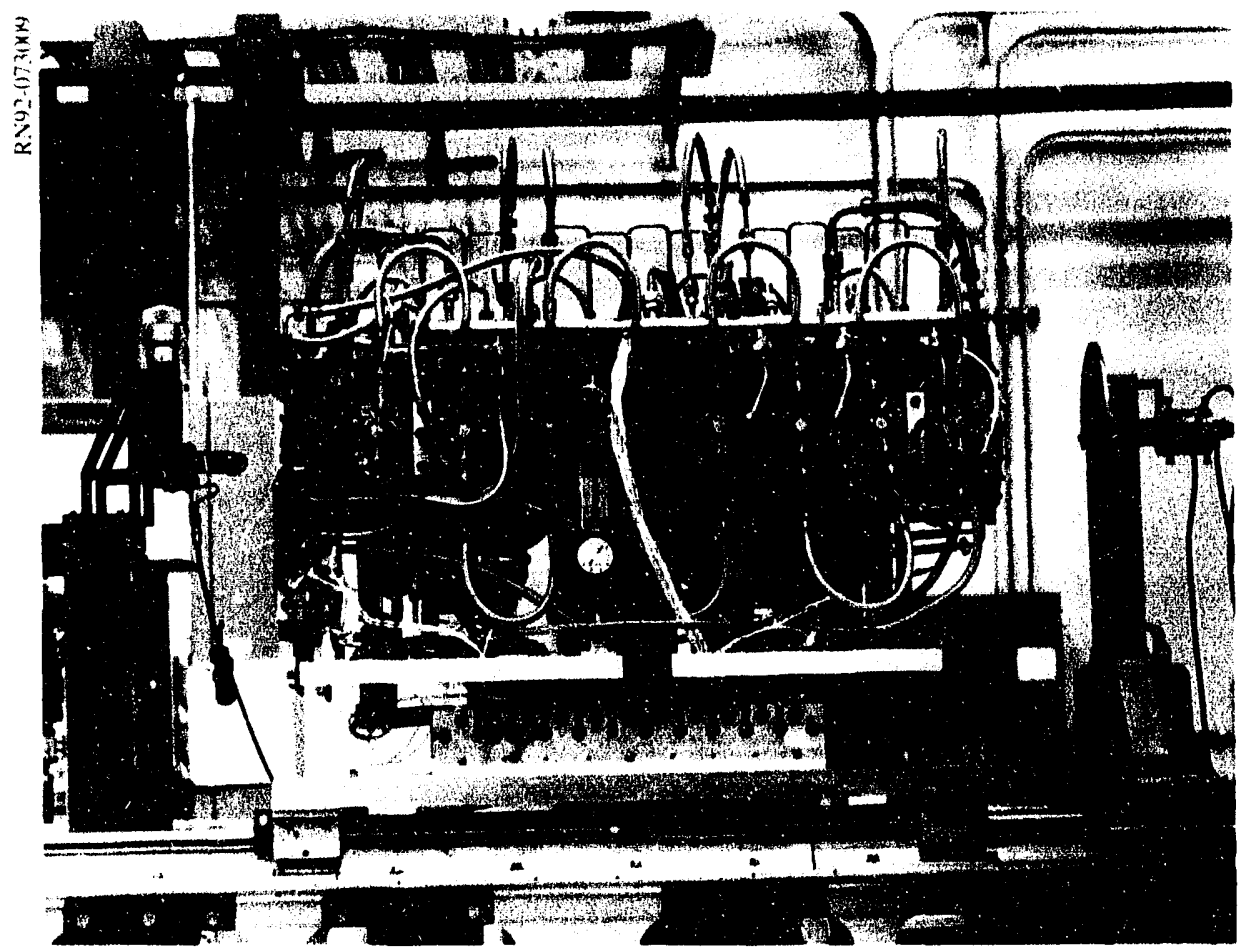

be operalting as expected through the high-power conditioning phase.

Figure 4.1 shows module \# I completely assembled before installation into the DTL vacuum vessel for heam experiment 2A. Module \#1 hats been operational on the GTA beamline for beam characterization experiments since early September 1992. This module has been operating as expected with no apparent problems. Figure 4.2 shows 8 of the 10 DTL modules in various stages of assembly to indicate the complexity of the assembly process as each module is built up to be fully operational.

\section{Waveguide Basement Floor Analysis and Installation}

Upon completion of experiment $2 A$, we will move the GTA experiment from the tunnel's northeast corner to the south side of the tunnel and position il directly over the waveguide batsement. Consequently, we significantly modified the existing tunnel floor dynamic-input spectrum. Because the acelerator will now be supported on a suspended stronghack, we are confronted with additional design challenges. Initially, the DTL, accelerator was designed for a dynamic-input spectrum in the previous, more benign, stable location. In its new location, it will be exposed to more background lateral vibration sources (c.g.. pumps and compressors) than the original, conventional support structure was designed to accommodate. As a result, the floor was latterally isolated from the north wall, but still is able to support vertical loads.

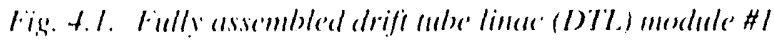

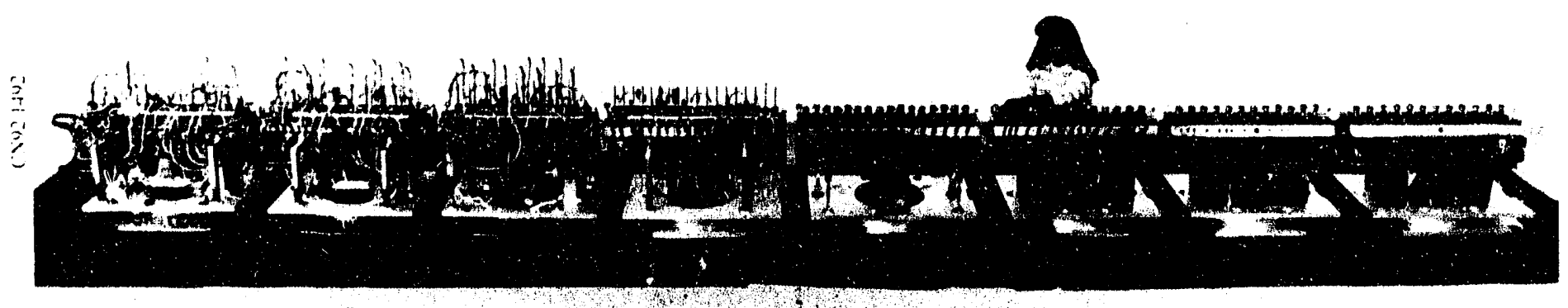

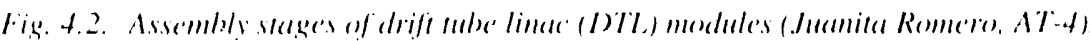


AT-t and MEE .. hald initially developed a finite e ment model of the DTL. assembly from the base of the module support pedestal to and including the drift tube mounted into the DTL module. Following vibrationai analysis. We found this mode' $s$ drift tube displacements were very small and could therefore be neglected. MEE-13 measuresis the dynamic response of the floor around the wareguide basement in 199? and updaces the dynamic model to include the new strungback. It was then fou do that the vibrations at the interface to the hasement s north wall caused larger drift tube displacements than the prevous moder had indicated. Beam dyna nic analyses showed that these drift tube displacements produced encugh of a stecring error to give an inaleceptable apparent emitlance growth. These simulation efforts identifited the level of vibration isolation reguired. such that the resultant inputs to the base of the support pedestal are at or tolow the original design environment. In addition, a modal survey was done using DTL module \#4 to verify the finite element model results. These results indicated the strongback needed to redistribute loads while maintaining the refuired alignment during accelerator operation. The structure had to be stiff enough to preclude deflections during operation at high vacuum after alignment at atmospheric pressure. During high-vacuum operations. the $60(0) \mathrm{lb}$ vertical up load at eacl of the 10 DTL pedestals must be balanced by the $6(0.0()()$ lb vertical load distributed over the vacuum vessel for A siructural finite element model was devel- oped to validate the resultant design. Figure 4.3 shows one of the three waveguide floor modules before installation into the GTA tunnel. The numerous cutouts shown provide access for $\mathrm{rf}$ waveguide and utility feedthroughs. The three floor sections were delivered in August and have been installed into the GTA tunnel. The numerous cutouts shown provide access for rf wiveguide and utility feedthroughs. The three floor sections were delivered in August 1992 and have been installed in the GTA tunnel.

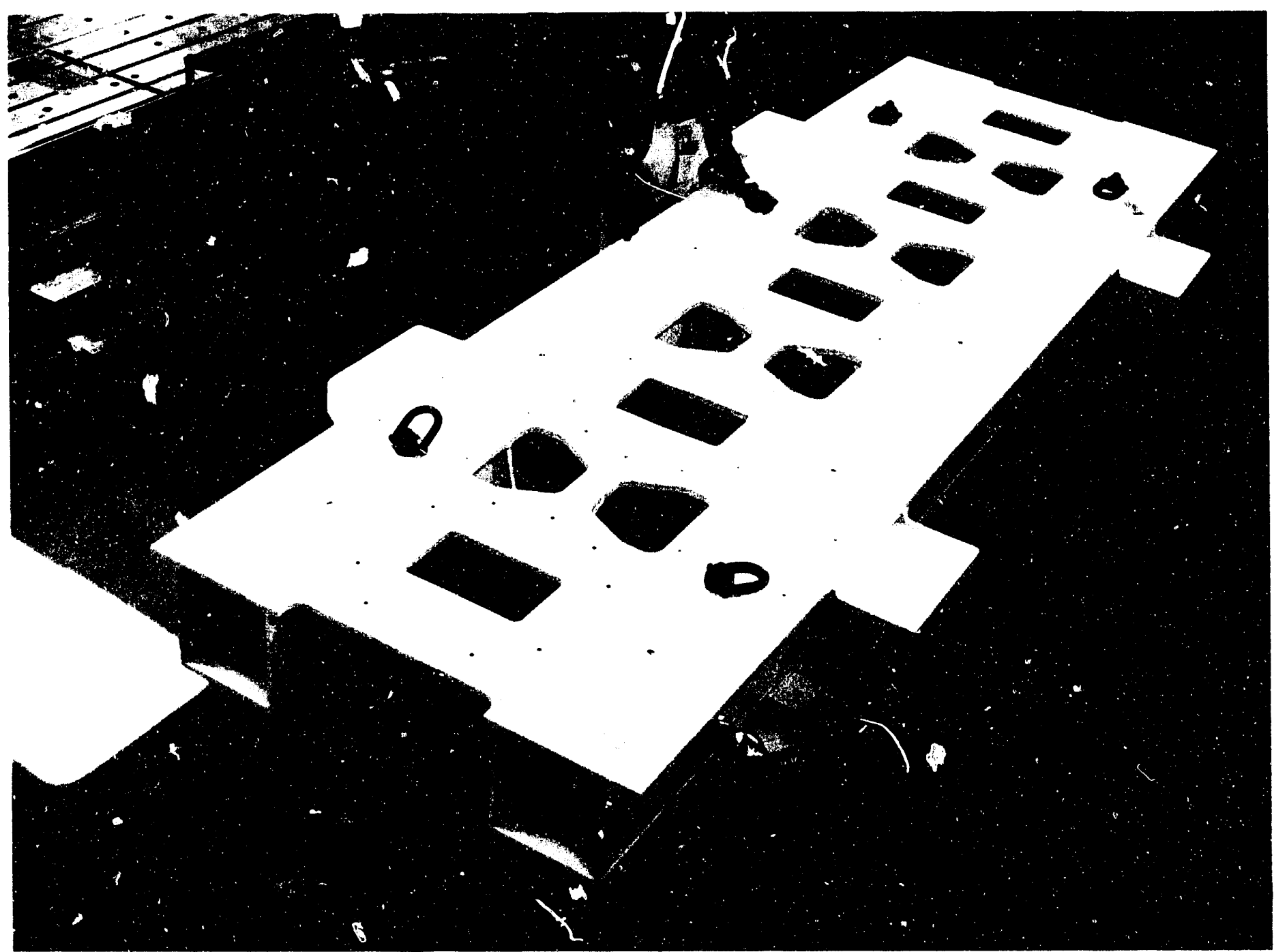

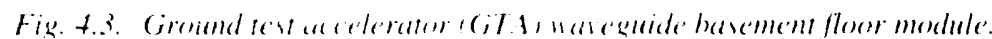




\section{Optics Design}

The GTA optics design went through several changes again in FY 1992. including conceptual designing, costing. and scheduling. At the beginning of the fiscal year, efforts were directed loward designing and building a "space qualifiable" (optics system, meaning the hardware would be designed for a spate mission, but not lested. However, if funding were to become available, the system could be tested and flown. Consequently, we crealled a conceptual design of the system and huilt preliminary thermal, structural, and dynamic finite element models. The thermal model determines both spatial and time-varying temperature profiles of the space structures and their magnets, including the on-orbit envirommental heal fluxes such as solar, albedo, and the earth's infrared. Dynamic and structural models were built using heam and shell elements, respectively, to interface with the existing Titan III model McDomnell Douglats established for the neutral particle beam space experiment (NPBSE) in 1991. These models enable parameters such as mounting schemes to be optimised for minimized weight. Much of the required interface data such as geomeric relationships fo other spacecralt hardware, internal heal sources and weights. and latunch and space envirenment dynamic loads were developed from past reports (e.g.. NPBSE. NPBIE).

During the liseal year the design focus changed 60 match the actual program funding. The change eliminated the term "space qualifiable" from the hardware description and replated it with "spake congnizant," meaning the optics system would look as if il could go into space, hut there would be no analysis to support the design. The high-energy heam tramsport (HEBT). eycepiece, and steerer would he designed as ground experimental hardware only and would not be required fo be space compatible. The change eliminated the term "space cognizant" from describing the lelescope. The bend. shown in lig. 4.4. was still forematin ats a sace compat- ible item and was designed as a pseudomonocopue or truss structure. This design addressed and mel its goals: an eflicient lightweight structure (o) carry flight and launch loads. maintain the precise magnet alignment requirements, maintain the particle beam axis in line with the structural axis to reduce high-interface stresses from eccentric load paths, provide maintenance and acessibility to the beam diagnostics and magnets, and have a reasonable assembly and commissioning plan.

The decign change resulted in us splitting the optics program into two paths. The first direction was to design the 24MeV ground telescope and eliminate the bend entirely, which would leave the GTA accelerator on the north side of the tumnel facing west. We completed a work breakdown schedule and cost estimate to determine if the hardware and labor savings would office the utility and facility costs associated with leaving the experiment in its present location. The second direction was to go back to a space design to support the far-field optics experiment (FOX) headed by Grumman with team members from McDonnell Douglas and LANL. The FOX is a 5-MeV "straight shooter" with a single joint enabling the telescope to be folded for storing dur- ing latunch. Consequently, this effort involved making layouts and pertorming preliminary dynamic and thermal an:alyias.

After the cost estimates and tradeoff analysis were completed, we decided the GTA experiment would be moved to the south wall where it would face east. Four other optics systems were investigated to help reduce the overall cost and schedule. The first was an 8.5-MeV ground "straight shooter," which would follow after DTL module \#5 was considered, eliminating the cost of installing the five remaining DTL modules and delaying the bend design and fabrication until a later date. The telescope would be designed so it could be upgraded to a 24-MeV configuration. The second optic system considered was to design both an 8.5 $\mathrm{MeV}$ bend and telescope and install them after DTL module \#5, with both assemblies potentially upgradable to $24 \mathrm{MeV}$. The third design '."suld be an 8.5-MicV space apiics system, which would com ete with FOX for funds set aside for a space experiment. The fourth system involved designing a 24 MeV ground system that would be installed after the initial DTL experiment when funds became available. After evaluating design configurations, cost estimates, future plans, and sched-

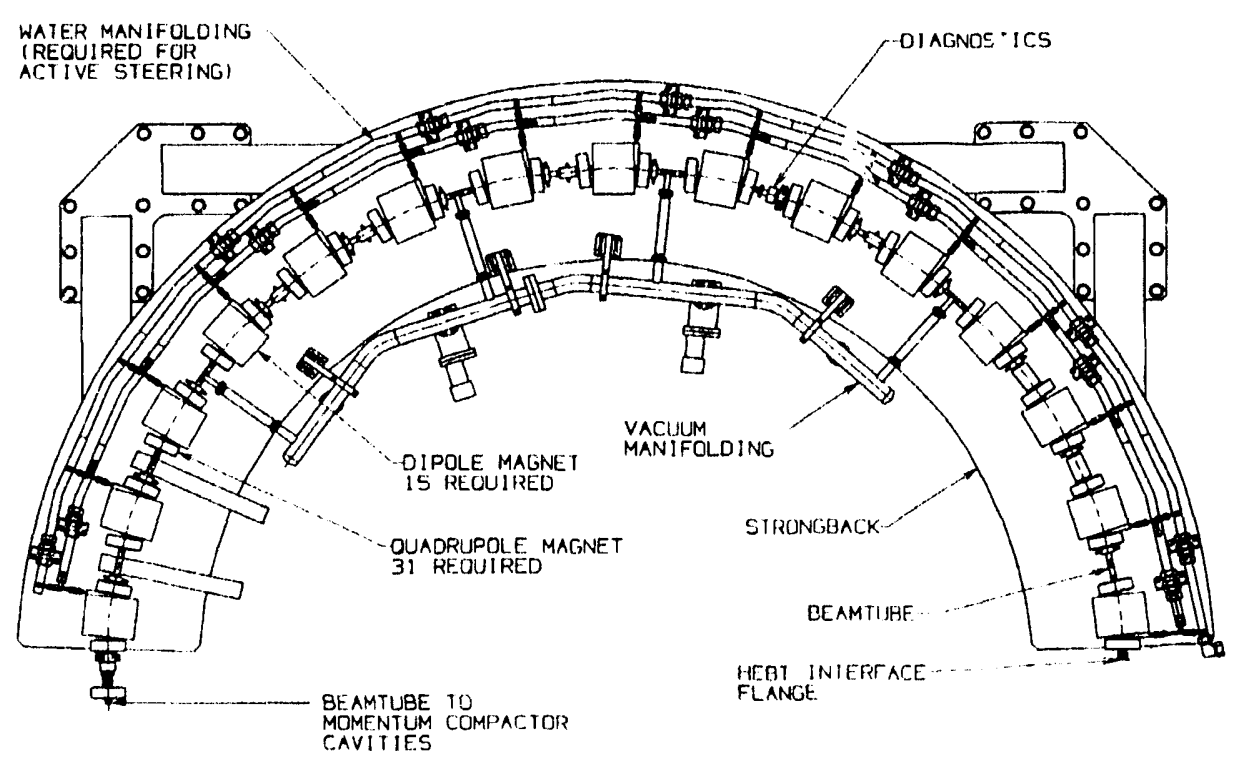

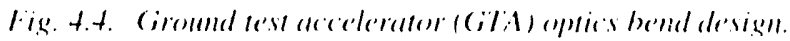


ule impacts, the fourth choice was chosen as the program direction.

Once the program focused on the 24$\mathrm{MeV}$ ground optics system, we completed a conceptual design of the bend. After reviewing the available tunnel layout. vibrationally unstable floors, and the logistics of multiple set-ups, an active alignment scheme using heodolites did not seem feasible. Therefore, the design presently evolving uses tight tolerances, a quadrupole assembly fixture with taut-wire alignment apparatus, and a coordinated assembly plan to ensure the bend magnets are properly aligned.

\section{Design of the Accelerator for Production of Tritium (APT) and Transmutation of Waste (ATW)}

Since the conception of LAMPF, the greatest challenge for the linac designer has been to engineer high-current, continuous-duty machines: several of our present studies involve such machines. During 1992, AT-4 led the engineering design, under a DOE study contract, for a 200-mA, 1000-MeV, cw, water-cooled machine called the Accelerator for Production of Tritium (APT). This work has required that we develop new cavity structures, and solve special cooling and maintenance problems, setting the stage for other machines of a similar nature.

A conceptual design for the APT accelerator has been in progress during FY 1992. This accelerator consists of a pair of $100-\mathrm{mA}$ proton sources feeding two RFQ's that operate at a resonant frequency of $350 \mathrm{MHz}$ raising the beam energy to $7 \mathrm{MeV}$. The beam is then delivered to DTLs designed for 350 )$\mathrm{MHz}$ transmission, further raising the energy to $20 \mathrm{MeV}$ before the two beams are funneled into a single 20()$\mathrm{mA}$ beam with a microstructure frequency of $700 \mathrm{MHz}$. At this point. a new accelerating structure is evoked, called a bridge coupled DTL (BCDTL). to carry the beam to $100 \mathrm{MeV}$. At 100 $\mathrm{MeV}$ the beam power is 20-MW cW and must be handled with extreme care.
The beam is injected into a sidecoupled linac structure similar to LAMPF where the beam is accelerated to $1 \mathrm{GeV}$ before it is directed to the tritium production targets.

While this study involves the concept of using a machine to produce tritium, with minor adjustments in output energy and current, the machine can be used to treat radioactive waste (i.e., Accelerator for Transmutation of Waste [ATW] or Accelerator Based Conversion $(A B C])$ or act as a rieutron factory of unprecedented flux. It is, therefore, a design with wide ranging applications. The basic design, however, is water-cooled and conventional in nature as compared with more exotic superconducting approaches.

\section{Mechanical Design}

One of the key decisions we made early in this study was to use electromagnetic quadrupoles in the DTL to resist radiation effects. Even small losses in a $200-\mathrm{mA}$ proton beam at

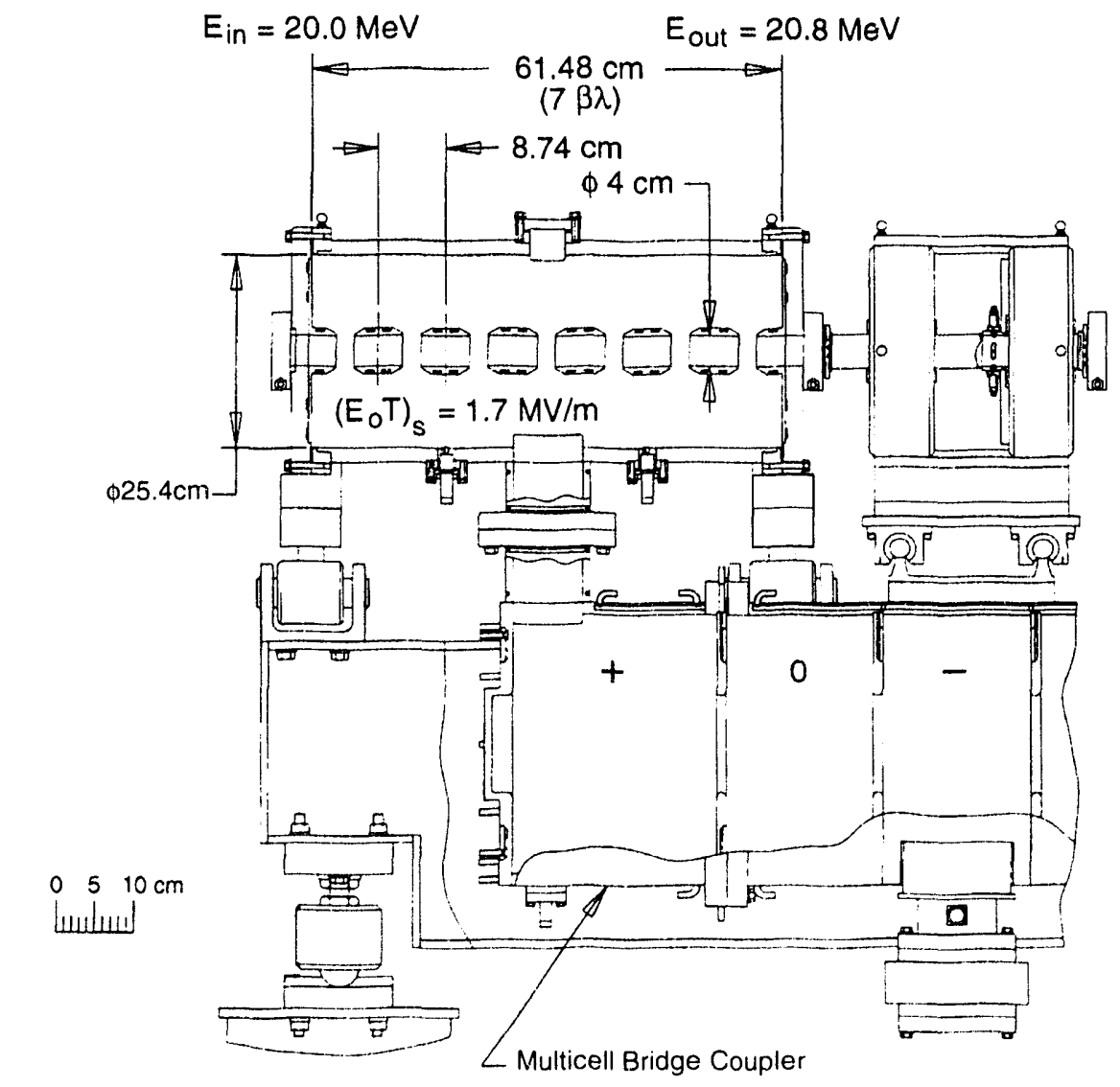

Fig. 4.5. BCDTL 700-MHz accelerating tank at 20 MeV. elevated energies will produce sufficient neutrons above a few MeV to significantly shorten the lives of rare earth cobalt-magnet materials. Using electromagnetic quadrupoles in the DTL, which dictate the minimum size for the DTL drift tubes, coupled with the need for continuous-duty rf sources, resulted in our choosing the $350 \mathrm{MHz}$ for the front end of APT.

Following the funnel at $20 \mathrm{MeV}$, we identified a more difficult problem: the need for low-energy, large-bore, highfrequency $(700-\mathrm{MHz})$ structure to accelerate the beam to its final energy. It was evident that a standard coupled cavity linac (CCL) would function well, but only at 80 or $100 \mathrm{MeV}$ and above. To bridge the gap between 20 and 100 $\mathrm{MeV}$, we needed an entirely new structure to avoid the structural, cooling, and operational problems of a very lowenergy $\mathrm{CCL}$. We developed the BCDTL for this purpose (Fig. 4.5). It consists of short accelerating modules, seven beta-lambda in length, which the 
beam dynamicists find give excellent beam transport results. We placed quadrupole doublet focusing magnets for strong focusing between modules at short intervals. Each of the BCDTL. aceelerator tanks holds six drift tubes. unique in that they do not carry quads. In addition. the drift tubes have extremely large bores considering the high frequency at which they must operate. Because there are no quads in the drift tubes, alignment folerances can be more lenient $( \pm 0.5 \mathrm{~mm})$. Consequently, the drift tubes can be "hard socketed" for good if seals, which are necessary for ew operation. The aperture ranges from $4 \mathrm{~cm}$ in diameter at 20) MeV to $5 \mathrm{~cm}$ at 100 MeV. It is desirable to achieve a high-alperture ratio of bore diameter $t o$ beam diameter $t o$ prevent beam losses and radioactivation by the beam. The BCDTL effectively overeomes the problems caused with an equivalent CCL at the same low energies by climinating the close-spaced of surfaces. multipactoring, and other operational difficulties. Also eliminated are cooling and fabricating problems, and incfficient of operation result- ing from the inherent low-shumt impedance of a CCL. struclure below 1(1) MeV. Figure 4.6 shows a lour-lank assembly of a BCDTL module.

Once the beam energy has heen raised (o) $100 \mathrm{MeV}$, a conventional pi-mode CCL is the best structure to use. The shunt impedance is now higher than a BCDTL and the fabrication and cooling problems are greatly diminished. A precedent also exists in L.AMPF for CCL structures fabricated at this entry energy, although at 70) $\mathrm{MHz}$, the APT cavities are $1.5 \%$ larger than L.AMPF and marginally difficult to braze. This factor is another reason why the choice of 3.50 and $7(0) \mathrm{MHz}$ was made for APT. Below 700 MHz, a CCL becomes difficult to build because of its increased size and bulk. The frequency choice that was selected is ideal from all engineering, cavity structures and labrication standpoint.

The 10()-MeV CCL module, shown in Fig. 4.7 represents over $3(0)$ such modules in the CCL., which compose $9(0 \%$ of the bulk and cost of the APT linac.

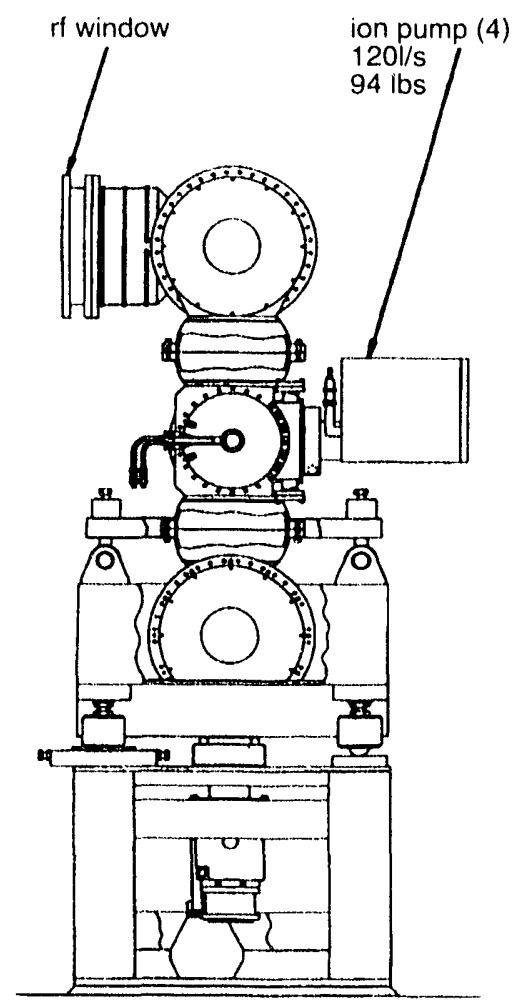

Fig. 4.6. Bridge coupled drift tube linas (BC TITL) module at 20 MeV.
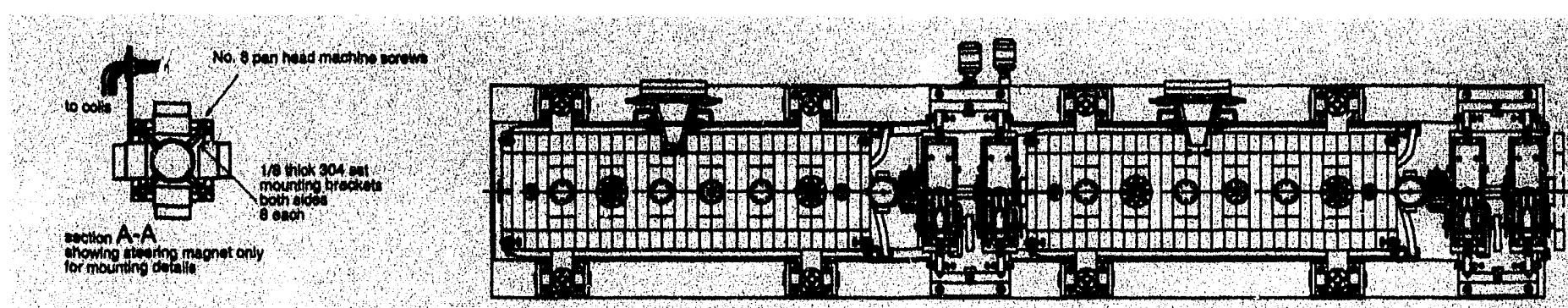

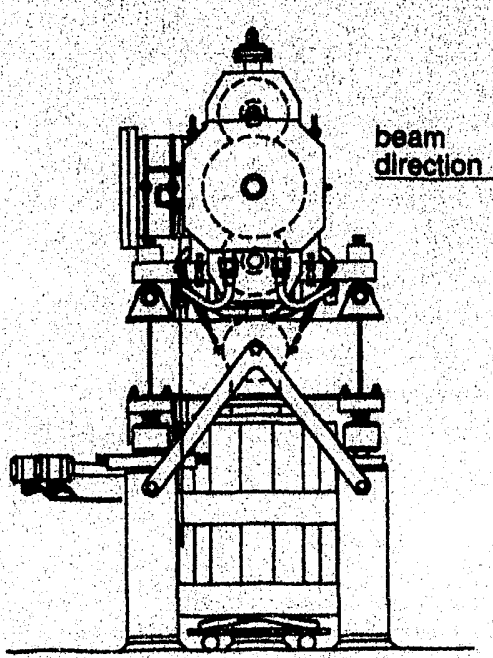

Fig. 4.7. CCL Module at $100 \mathrm{MeV}$.
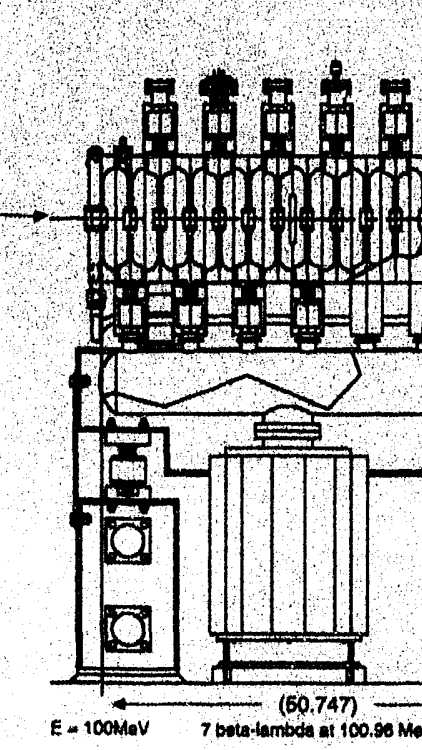

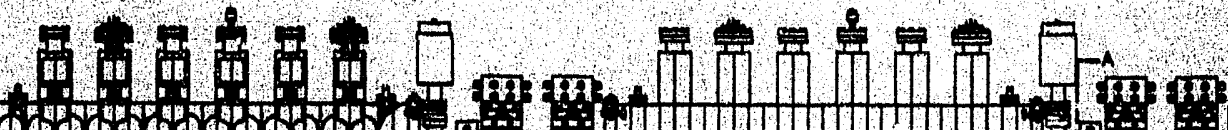

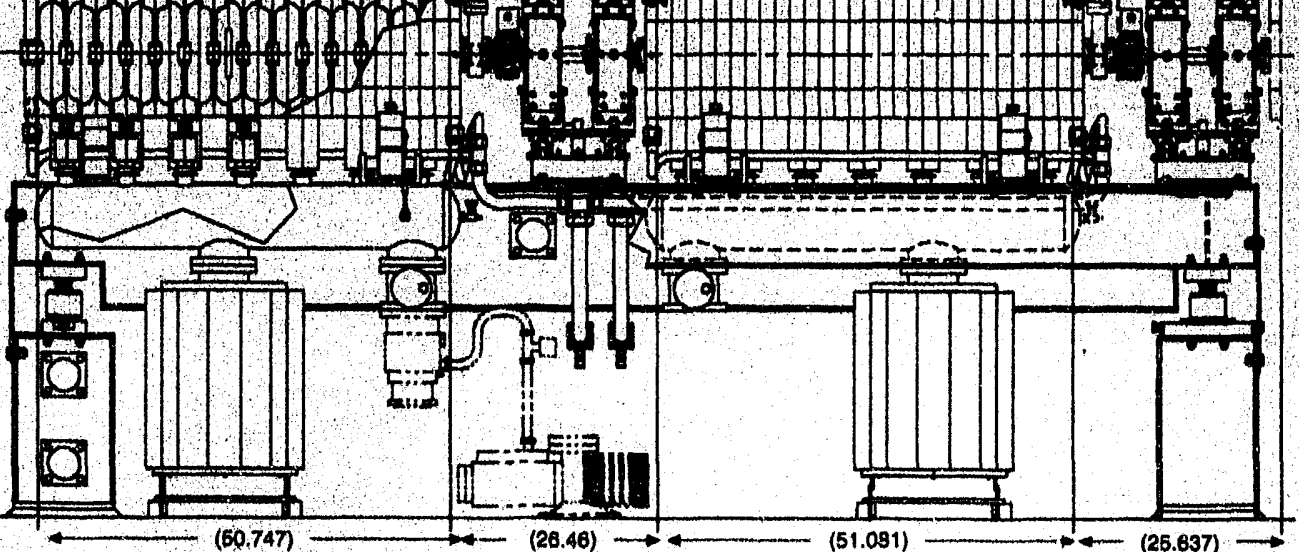

3.5 bota-lambeda at $101.73 \mathrm{MoV} \quad 7$ betatambda al $102.52 \mathrm{Mets}$ 


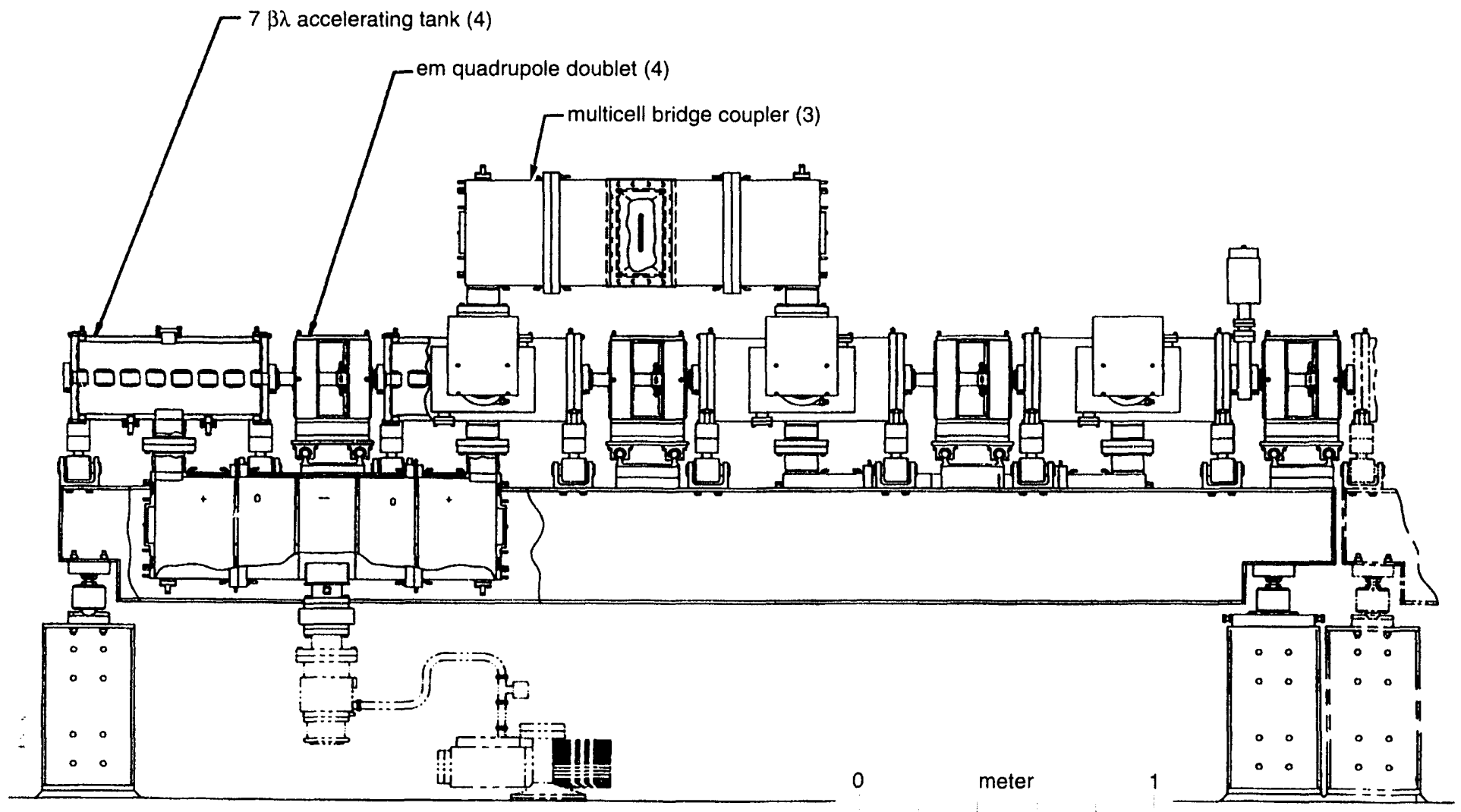


This structure is designed around a single half-cell concept in which half the coupling cell and half the accelerating cell are machined into the same forging. This is a departure from the technique used at LAMPF in which the coupling cells were brazed as separate assemblies. However, by splitting the accelerating cells along the septum midplane, it is now possible to machine adequate cooling channels to accommodate $\mathrm{cw}$ operation of the structure because septum rf heating is of primary concern in a cw CCL. Along with the septum cooling channels, an internal channel is also machined around the coupling cell periphery. A cross section of the resulting septum cooling channels is shown in Fig. 4.8. The entire cooling system for a seven beta-lambda long structure consists of longitudinal gun-bored cooling channels around the periphery of the tank assembly distributed in pairs. with each pair feeding a single septum and coupling cell. The flow is mass balanced and confined to counterflow, which results in a uniform temperature distribution along the length of the tank. Development of this cooling scheme along with the BCDTL. constitute two of the major engineering breakthroughs in the APT linac design.

A third significant design development is based on the potential need to perform maintenance on beamline components under radioactive conditions, which demands quick or even remote servicing. We found the principal components subject to failure are the vacuum pumps, the rf windows, and the intertank beamline components, which include the doublets. diagnostics, steering dipoles, bellows, and a rad-hard vacuum valve. All the intertank hardware is mounted on a pair of rails for quick removal and ease of alignment as shown in Fig. 4.9. The hardware is constrained of fit into a longitudinal space of 3.5 to 5 belia-lambda depending on the energy point heing considered. The unit can either he fully disassembled on line. if conditions permit. 11 replace field coils, or it can be partially disassembled to changeout valves or bellows. It can also be removed en- tirely and taken to the lab for total overhaul, perhaps in a hot cell. A replacement unit, prealigned in the lab and completely checked out, could then be slid onto the rails providing predetermined alignment with the asinstalled hardware.

The entire APT is designed to be modular in that each klystron amplifier drives a segment of the accelerator using from $600 \mathrm{~kW}$ to $1.25 \mathrm{MW}$ of total power. Each accelerator module is mounted onto a rail system complete with a self-contained vacuum system, water-cooling manifold, focusing system, diagnostics and essential instrumentation. It is prechecked, aligned. and $\mathrm{rf}^{\prime}$ conditioned offline and then transported to the machine tunnel where it is installed on prealigned kinematic mounts. This modular approach allows the machine to be maintained either in sections, off line, or removed as a complete unit for major repairs.

The general APT design described above is applicable to any proposal for a water-cooled, funneled machine in the $30(0)-1090(0)-\mathrm{MH} z$ range that accelerates protons to energies of 1 to 2 $\mathrm{GeV}$, operattes at beam currents up to $350 \mathrm{~mA}$, and functions in a cw mode. This APT study has been a great engineering challenge, has placed us in a position to deal with similar machines for radioactive waste disposal, and has shown potential for future applications.

\section{Seven-Cell Superconducting Cavity and Superconducting Cavity Chains}

One of the most advanced superconducting cavity prototypes was designed and released for fabrication during FY 1992 after a collaborative effort between AT-4 and AT-1. The prototype consists of seven cells of high-

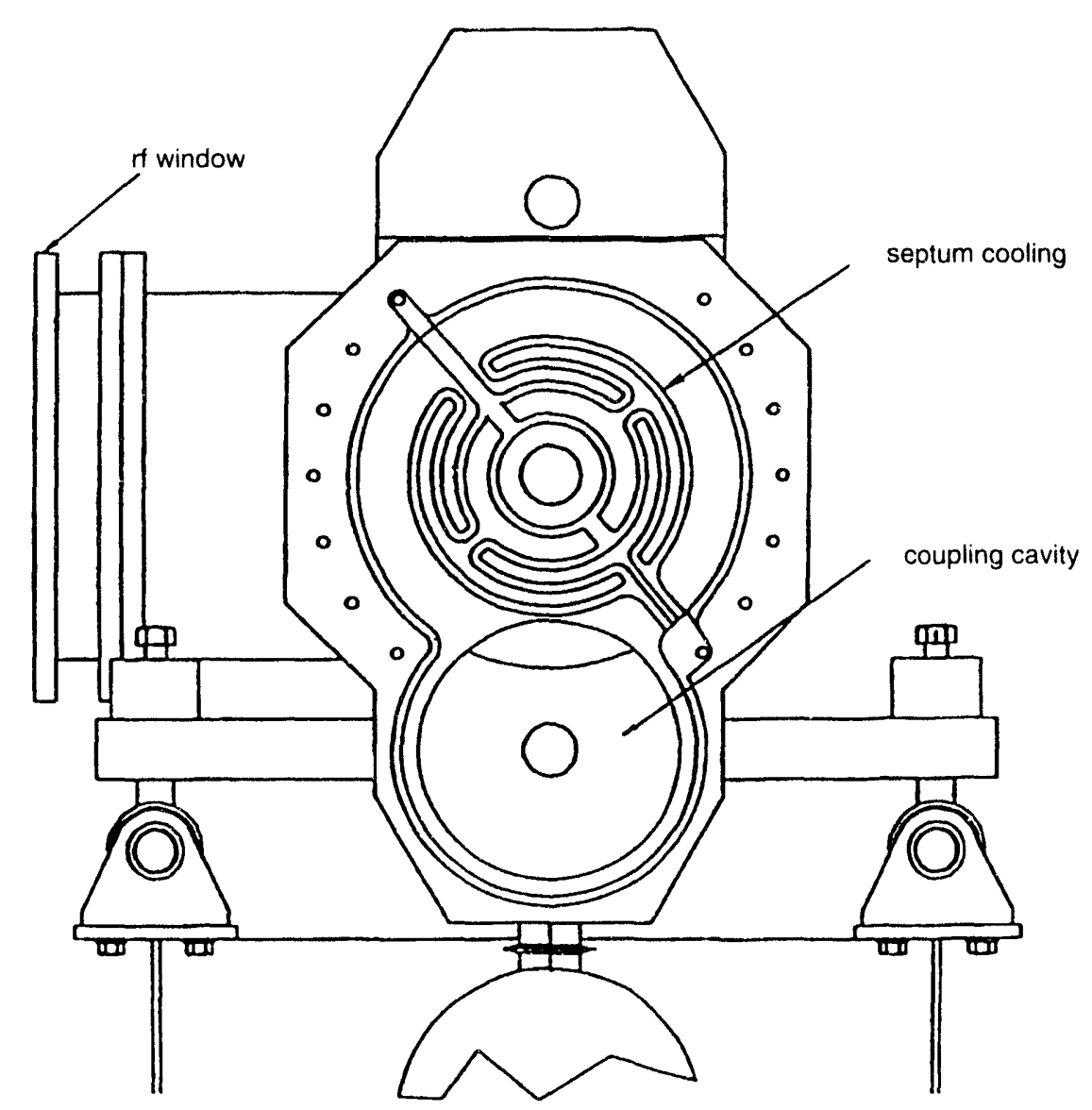

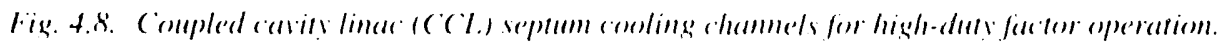



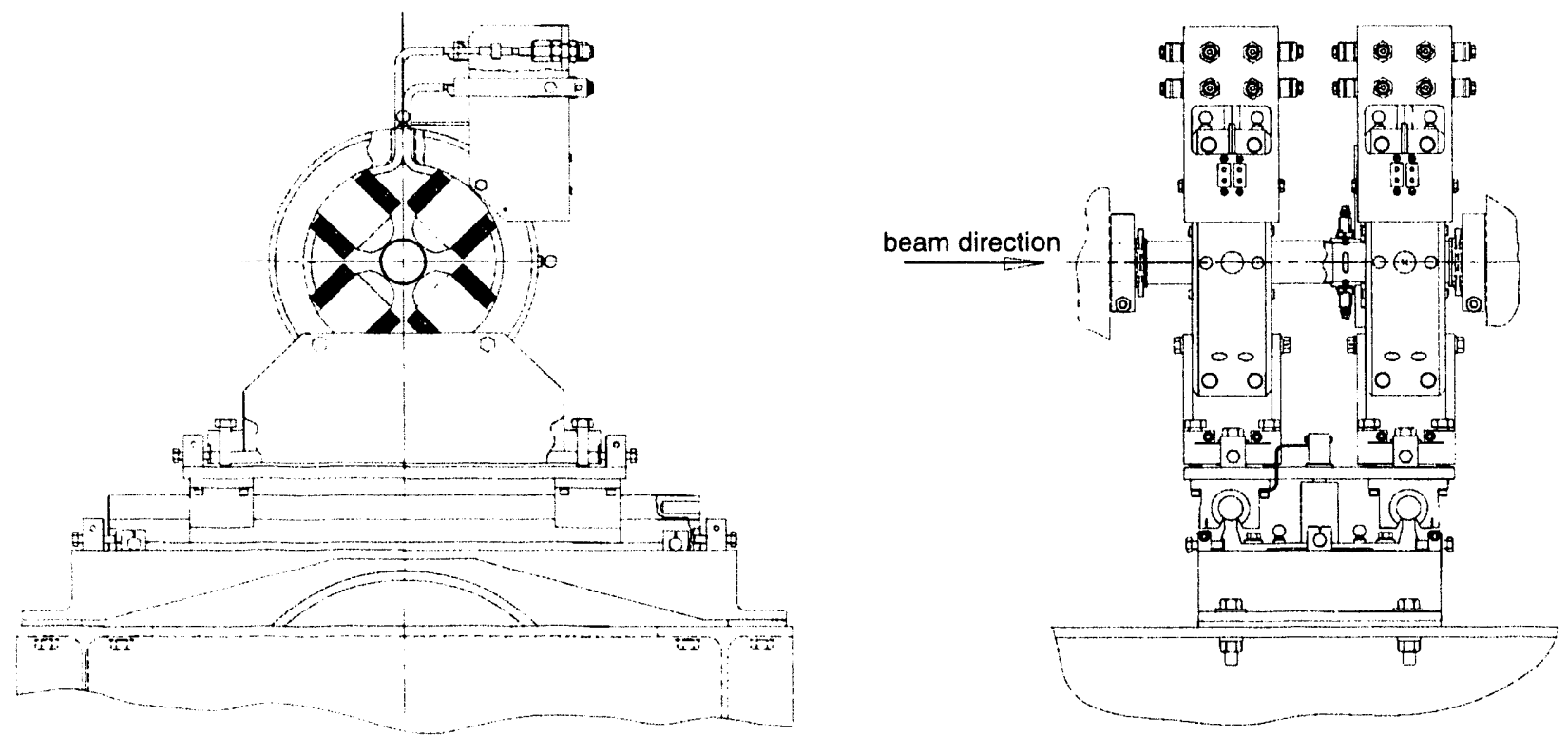

Fig. 4.9. Quadrupole doublet alignment and quick maintenance hardware for radioactive applications.

purity niobium welded together into a $805-\mathrm{MHz}$ cavity chain. Unique features of this cavity include its extremely high unloaded $\mathrm{Q}\left(5 \times 10^{9}\right)$ when operated at superfluid helium temperatures $(2 \mathrm{~K})$ and the high fields expected in the structure (12.5 to 15 $\mathrm{MV} / \mathrm{m}$ ) at this relatively low frequency. The $805-\mathrm{MHz}$ resonant frequency implies that a large surface area will be exposed to high field levels. The successful test of this prototype would be a benchmark in coupled cavity superconducting technology.

A representation of the seven-cell assembly is shown in Fig. 4.10. Individual cells are electron-beam welded at the equators and iris. The cavity subassemblies are then acid etched. tuned, and annealed at high temperature using titanium gettering to remove surface impurities. Cavity assembly inside the stiffening cage of six titanium tubes ensures that microphonic resonances exceed $240 \mathrm{~Hz}$ in the lowest transverse mode for adequate $\mathrm{rf}$ stability.

Also shown in Fig. 4.10 is a four-bar flex linkage for fine tuning. This linkage is expanded in Fig. 4.11. Fine tuning is accomplished by deforming the end half-cells of the cavity chain.

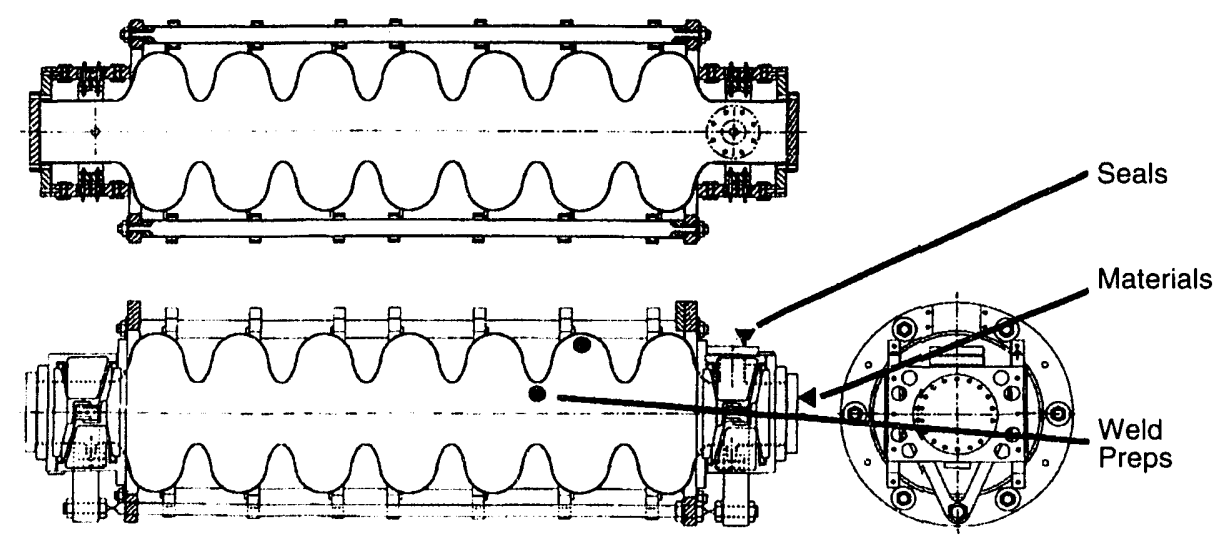

Fig. 4.10. Seven-cell high-gradient superconducting cavity showing areas of advanced technology:

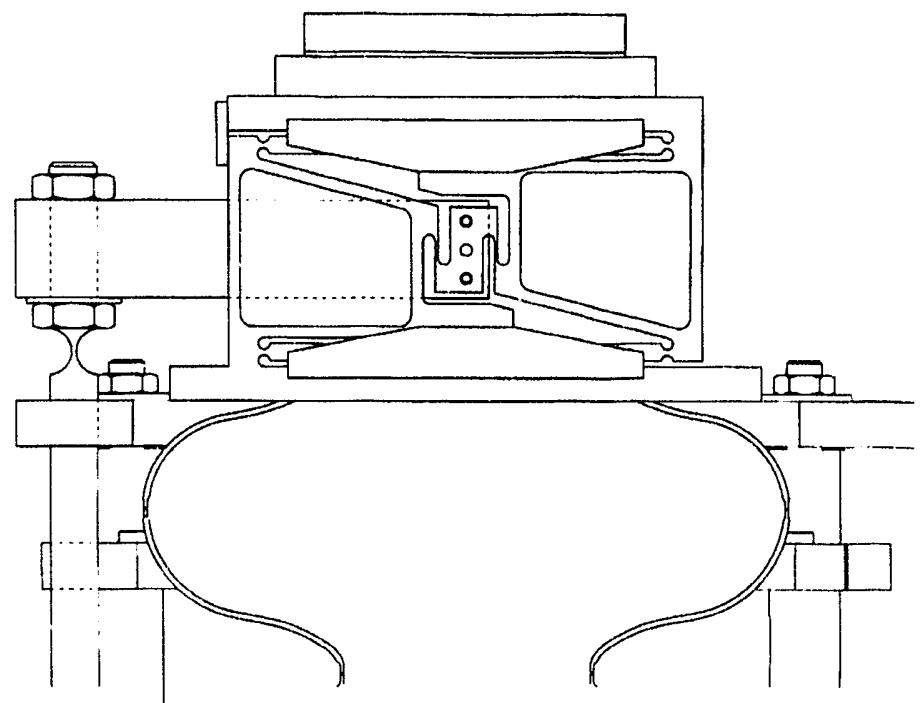

Fig. 4.11. Four-bar linkage used to achieve microtuning of the end half-cells of the seven-cell cavity. 
One must be careful to work within the elastic limit of niobium at superfluid temperatures; however, the tuning sensitivity of the system is almost 320 $\mathrm{KHz} / \mathrm{mm}$ and the elastic tuning range is $\pm 41 \mathrm{KHz}$. To achieve this tuning range, the adjuster compresses or expands the end cells by only $\pm 0.13 \mathrm{~mm}$. Because of the extremely high $Q$ of this cavity chain, the linear yoke motion of the actuator necessary to achieve a 1 $\mathrm{Hz}$ step in cavity frequency is only 250 $\AA$ (2.5 mm).

The seven-cell design departs from conventional engineering methods in several other ways, including the use of commercial metallic seals rather than indium. Indium is a good sealing material at superfluid temperatures, but tends to flake. Even the smallest particle falling into the cavity will destroy its high-field capability. To use the commercial Helico-flex Delta-Seal requires that niobium alloy flange material be selected with a Vickers hardness greater than 100. We selected an

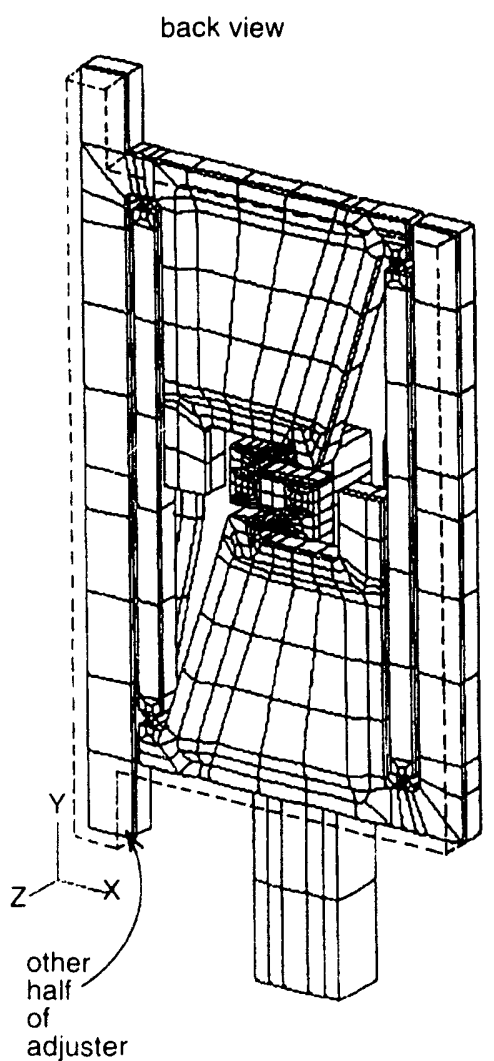

alloy consisting of $10 \%$ hatnium and $1 \%$ titanium in niobium and tested it for weldability to pure niobium and resistance to superfluid helium leaks.

The seven-cell design described above is a research cavity with various uses, including pion or proton acceleration at medium energies. It is scheduled to undergo rf testing at full power in FY 1993.

\section{Vibration Analyses}

Because of an inherent sensitivity to microphonics, superconducting cavities must be designed to resonate at relatively high frequencies, despite the low stiffness of the basic thin-walled niobium structure. To raise the fundamental frequencies of this type of cavity, proposed stiffening schemes must be analyzed to determine if the required fundamental frequency levels are achieved. Finite element models of several superconducting cavity structures have been built to determine their fundamental resonant vibration modes.

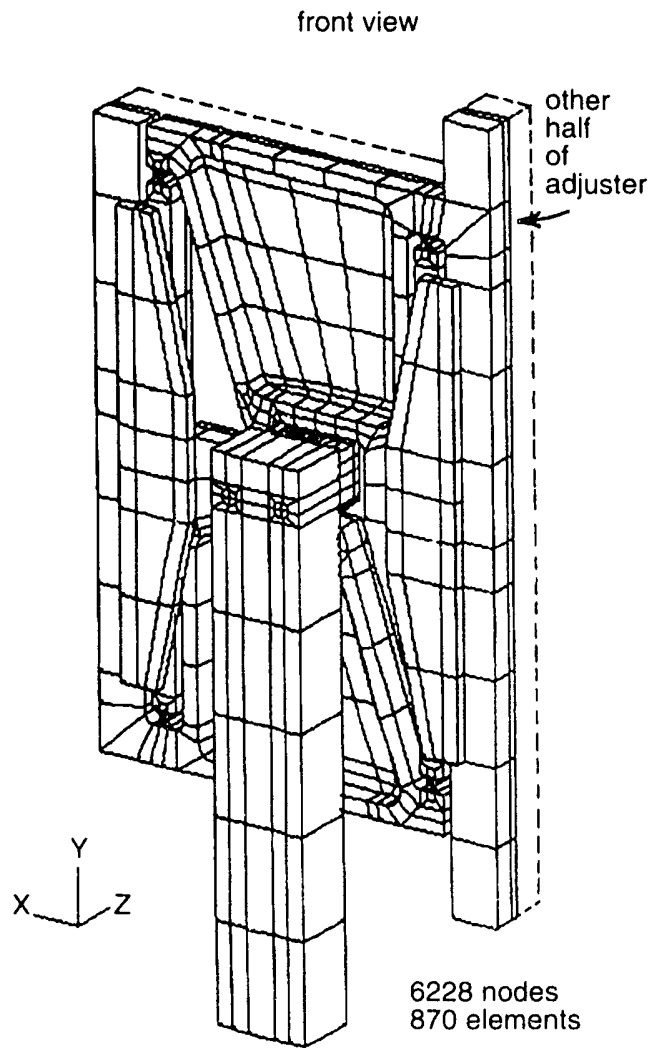

Based on a highly favorable comparison between the finite element model of a CERN/DESY four-cell cavity and actual vibration test data of that cavity, we analyzed the LANL seven-cell cavity. Various stiffening schemes were proposed and analysis was done to arrive at an optimum stiffened configuration. The resultant method for stiffening the rf cavity consists of a structure with six titanium tubes connected at either end with a bulkhead, to which each cavity cell equator is rigidly attached. This simple structure raised the first (lowest) transverse bending mode from $37 \mathrm{~Hz}$ to more than $240 \mathrm{~Hz}$.

Based on this success, we constructed a similar analytical model to represent a nine-cell cavity currently being developed at the Continuous Electron Beam Accelerator Facility, where a novel stiffening concept is being considered that encases the entire cavity within a thin-walled cylinder.

\section{Fine Frequency Adjuster Mechanism}

The seven-cell cavity 'fine' tuning procedure involves elastic deformation of the cavity"s end half-cells. We have designed an adjuster mechanism to effect this end-cell deformation via longitudinal translation of the cavity end flanges. It is mounted between the stiffening rod bulkhead and the cavity end flange (Fig. 4.11) and is similar to a four-bar linkage, but relies on flexures to eliminate the possibility of backlash and hysteresis. The adjuster is actuated through a yoke attached between two adjusters on either side of the cavity. Translation of the yoke rotates the center flexure, which in turn causes longitudinal expansion or contraction of the adjuster via a four-bar linkage motion of the outer flexures. A detailed threedimensional, finite element model of the adjuster mechanism was created (Fig. 4.12), representing half of one adjuster. Although the niobium cavity is not directly included in the model. spring elements are used to represent its stiffness. We analyzed several different loading conditions to explore the various operating environments. 
Recently, a modified version of the seven-cell fine-frequency adjuster mechanism has been adopted by Cornell's Newman Laboratory of Nuclear Studies for use on a single-cell superconducting cavity.

\section{Support of Others}

\section{Group N-2 FAA Moderate Energy Beam Transport (MEBT) System} Los Alamos National Laboratory's Group N-2 has purchased a 2.75-MeV RFQ from AccSys Technology Inc., which will be used in experiments to determine the feasibility of using a resonance absorption technique to detect nitrogen bearing plastic explosives. AT -4 was asked to design and fabricate a moderate energy beam transport (MEBT) system for use on the exit of the RFQ. The MEBT contained four electromagnet quadrupoles (EMQ) obtained from the Accelerator Test Stand project in AT Division. The EMQs were retrofitted with new mounting devices, which provided four degree-of-freedom precision adjustments. The mounting devices also incorporated kinematic mounts, which allowed for removal and repeatable replacement of the $100 \mathrm{lb}$. EMQs. A small $1-\mathrm{cm}$-bore permanent magnet quadrupole (PMQ) was mounted on the exit of the RFQ in a vacuum tight holder. Provisions were made to monitor beam current along the beam line using two Model 150 Pearson coils. The entire MEBT assembly was mounted on a support stand, which provided adjustment in the $x, y$, and $z$ axes. In July 1992, the MEBT was assembled and aligned at AccSys Technology. Acceptance testing and operational experiments are currently being conducted by N-2 personnel. Figure 4.13 shows the MEBT assembly currently being used by $\mathrm{N}-2$.

We are currently designing a new MEBT for the next phase of this program. It will include a $90^{\circ}$ bend in the -Y direction, eliminate two of the EMQs, incorporate a new support stand, and add diagnostics in the vertical leg. The new accelerator and MEBT will be installed in the UHTREX facility at LANL's TA-52.

\section{Group N-2 Nondestructive Assay Barrel Scanner}

AT-4 persomnel assisted Group N-2 in the design of a combined thermal/ epithermal neutron (CTEN) nondestructive assay diagnostic. This instrument will be used to interrogate TRU waste and provide the required

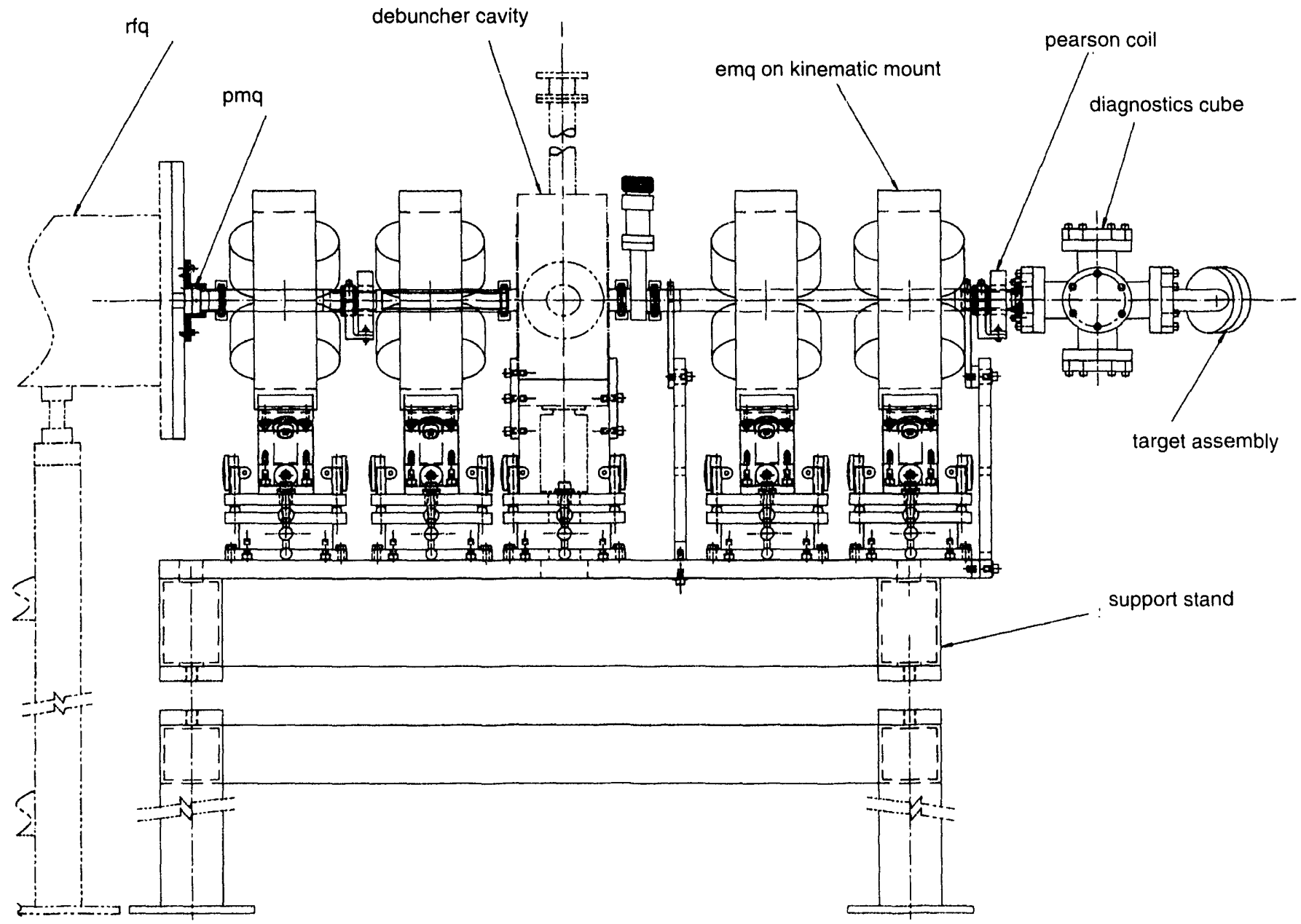

Fig. 4.13. Moderate energy beam transpon (MEBT) system assembly: 
characterization for proper disposal. The device will be fabricaled and commissioned at N-2 before shipment to the Ilaho National Enginecring Laborafore for tinal installation.

The CTEN diagnostic is the latest version of a long line of neutron counting devices, and the design is very similar to carlier versions. Design changes have been limited to the addition of extra shielding and to upgrading the instruments and mechanical drive trains. The most significant design change has been to replace the drive trains used (1) manipulate the target and shielding door from chain and right angle gear drives to modular rodless cylinder units with servo motor controls. The replacement of hand assembled drives with modular units allows for significant savings in the amount of design detail required and in the assembly and ad-

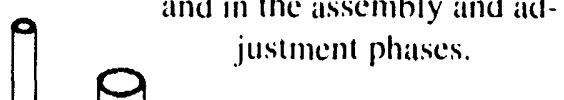

We have completed the design of the lop alssemblies and work is progressing (on the details of the shiclding doof and target positioning assemblies. These design modifications are intended to minimize the amoumt of time recpuired for redesign and use the skilled fabricalion craltsmen available al N-2.

\section{Group MP-7 LAMPF Support}

\section{Helium-je't Experime't!}

AT-4 is assisting MP-7 in an engineering and design effort in support of a LAMPF helium-jet experiment. This experiment requires a coaxial cylinder arrangement, which is shown in lig. 4.14. A maximum of 10 allm of helium gas is introduced at the center of the left end of the coaxial assembly of concentric cylinders, expanding through the cone lo an 8 -inch-long chamber filled with vertically mounted test foils. The helium is expected to flow parallel to the test foils in a laminar condition. At the right end of the 8 inch flow chamber is an array of capillary tubes that will extract the helium isotopes produced when the assembly is introduced into the I,AMPI heamline at the $A-6$ largel area. The annulus between the coaxial cylinders contains conling watcer.

This assembly hats the capability of moving +2 inches about the center of the $x$-inch lest chamber in the $x-y$ direction and normal to the beam.

C'urrently the design is completed with $50 \%$ of the hardwate in procurement. A moxk-up of this experiment is scheduled to start in early 190.3 in the l.AMPl: hol-cell areal. After complelion of the mock-up and off-heam testing the assembly will be installed in the A-f larget areal. Subsequent to the first experiment. We plan ib design fest chambers that can he quick y removed and reinstalled with new foils.

\section{A-2 Targer Area lpereade}

Personnel in $\wedge \mathrm{T}-4$ are also assisting in the upgrade to the $\wedge-2$ larget area at 1.AMPF. Redesign work is in progress to replace some existing hardware in the targel area, including vacuum tubes, collimaltors, and coolant lines. In the process of replacing this hardware, we are improving the current method of extracting heat from the target arcal. To support this redesign effort, we are developing several finite element analysis models lo analyze the target area's heat transfer characteristics based on the hearn's power deposition on the larget. Recommendations can then be made on the size and quantity of coolant channels necessary fo cool the collimators and vacuum lubes. 


\section{Environment, Safety, and Health (ES\&H)}

In the interest of improving the group's formality of operations, AT-4 has adopted a graded approach with an incremental implementation plan to accomplish desired changes. The AT-4 environmental, safety, and health (ES\&H) self-assessment plan has been completed, approved and implemented. Work is continuing toward accomplishing the goals outlined in the plan. Individual training plans for employees have been completed, and formal ES\&H training for all AT-4 personnel is in progress. A tracking and filing system is in place that includes employee job specific technical training as well as ES\&H training. We have continued to generate and resolve ES\&H Action Sheets throughout FY 1992. AT-4 has taken an active part in selfinspections as well as division inspections. During the Tiger Team inspection in August 1991, 225 ES\&H Action Sheets were generated. All but 20 have been resolved, and we are in the process of addressing these. Material Safety Data Sheet (MSDS) libraries have been established on the third floor of Building 365, in the group office, and in the drawing vault. In addition, a liquid chemical and chemically contaminated solid waste storage area has been established in Building 365 .

AT -4 has completed the standard operating procedures (SOP) and normal operating procedures (NOP) documentation for all testing operations within the group. Detailed SOPs and NOPs for the high-power cryo test bed, the low-power cryo test bed, the pressure and mass flow test stand, operation of the drawing print machines and plotters, and use and change out of anhydrous ammonia tanks were written and placed under quality assurance document control. Other documents included the test supervisors manual for If power conditioning and testing of DTL modules in the high-power cryo test bed.
AT-4 also introduced a QA controlled document system that includes a formal cover sheet, distribution control, and document change notice (DCN) control of all revisions to the document. We have used this control to formalize the issuance and revision of manuals and procedures that have been designated as needing better accountability.

The present AT-4 drawing vault is an advanced system with a print machine, CAD plotters, and computer links combined with microfilm, disk and hard copy archives as well as DCN records, computerized drawing lists, and classified document records. The drawing vault serves AT-3, AT-4, AT-5, and AT-10. By combining and making efficient use of this resource, these groups have realized a significant cost savings, improved quality, and better QA document accountability. The vault is an approved repository for classified drawings, documents, and CAD disks with a classification of Secret NSI or less. Proper marking, identification, and accountability of all drawing hard copies, document files, and disks is maintained. Issuing and tracking drawing numbers and DCN forms are required as well as maintaining uniform procedures, listings, and accounting records to prevent duplicates, lost originals, or damage to drawings, documents, or disks.

AT-4 has dramatically changed in the formality and awareness in which it handles ES\&H issues.

\section{References}

1. S. J. Black and G. Spalek, "Calculation of Mechanical Vibration Frequencies of Stiffened Superconducting Cavities," 16th International Linear Accelerator Conference, Ottawa, Ontario, Canada, August 23-28, 1992, Los Alamos National Laboratory document LA-UR-92-2735.

\section{Technical Memoranda}

The following AT-4 Technical Memos were written during the report period.

1. D. Liska, "Vacuum Analysis of the 20-100 MeV BCDTL," AT-4:9202, April 1992.

2. D. Liska, "Generalized Design Formulas for Low Energy Electromagnetic Quads," AT-4:92-03, April 1992.

3. D. Liska, "Vacuum System Design Cookbook," AT-4:92-04, April 1992.

4. D. Liska, "Extreme Limits of Operation for Compact Electromagnetic Quadrupole Field Coils, Including Poletip Performance and Maximum Focusing Impulse (<GL $>$ ) Generated," AT-4:92-05, October 1992. 

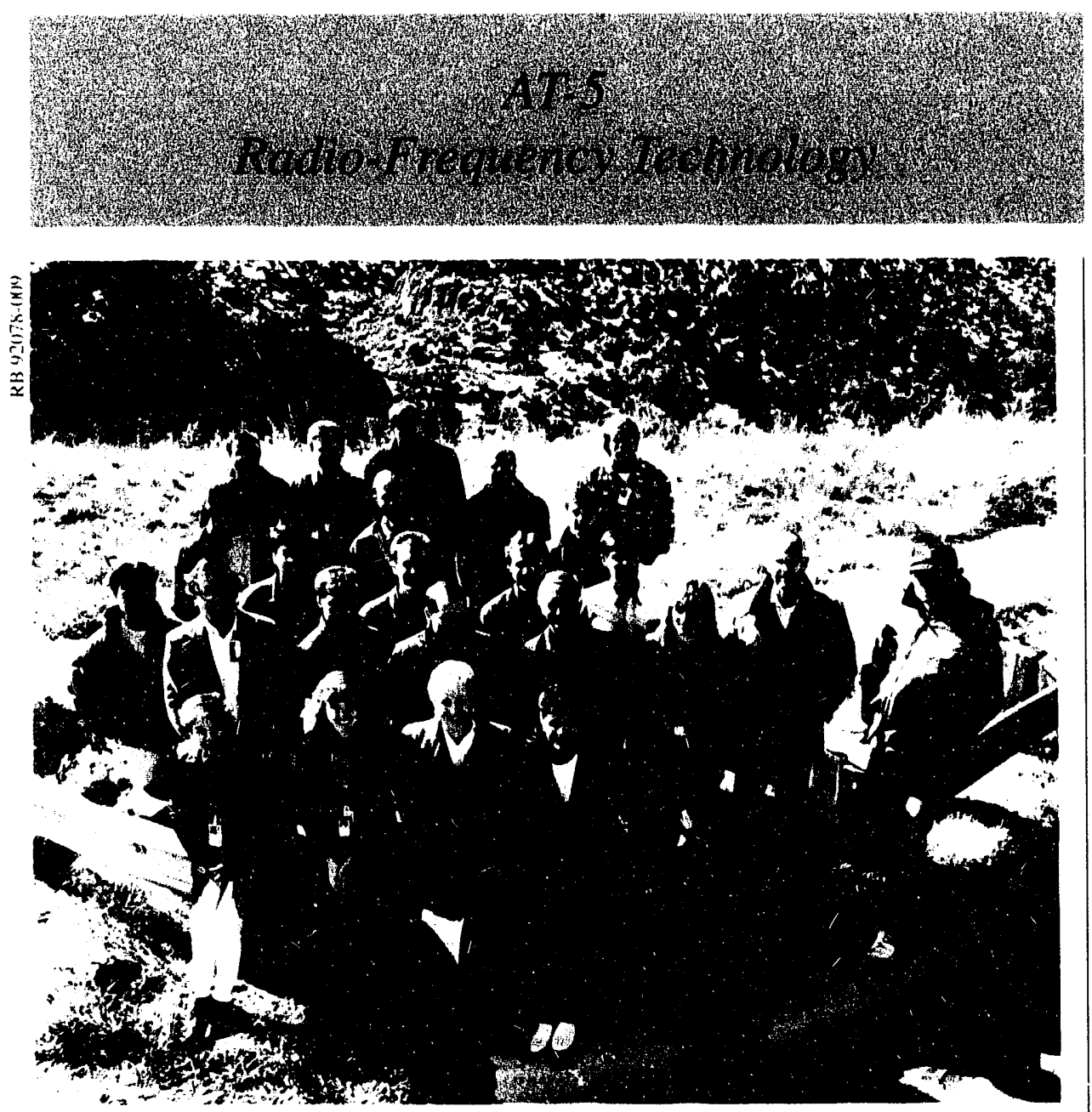

Introduction 6)

GTA 61

Overvicul......................................... 6 )

High-Poner of System Develoment and Production

RF Sistem Integration with GTA

Automated Kls:stron Test Stand ....64 RF Comtrols ................................ 64

Feedforward Module. .66

University of Thente 66

Boeing APLE 67

Argonne Advanced Photon Sontre (APS)

Superconducting Super Collider (SSC) Laboratory Linac

Directed Energy Weapons Poner Integration (DEWPOINT). 70

Plasma Soutre Ion Implantation...... 71

Grotroll .72

Accelerator Production of Tritium (APT)

Accelerator Transmutation of Waste (ATW) and Accelerator Based Comversion ( $A B C$ ) .73 


\section{Introduction}

AT -5 has a primary mission to develop high-power radio-frequency (rf) systems for particle accelerators. The key elements which make up the highpower rf systems are the high-power generators with their associated support equipment and the rf feedback controls.

The same expertise used to provide the high-power rf systems for accclerators is applicable to other areas. This provides AT-5 with other opportunities to develop technology and applications in radar systems, waste processing, and materials processing.

The following describes the programs and activities of AT-5 during FY 1992.

\section{GTA}

\section{Overview}

In FY 1992, AT-5 integrated the 425$\mathrm{MHz}$ of equipment with the first of the $850-\mathrm{MHz}$ hardware. This was AT-5's first opportunity to demonstrate the if system as it was designed and built and as it applied to an accelerator.

AT-5 was responsible for significant input toward developing the controls and computer interfaces as well as the four main components of the rf system for the ground test accelerator (GTA). The four main areas we were responsible for were the $425-\mathrm{MHz}$ tetrode amplifiers, the low-level rf (LLRF) controls, the $850-\mathrm{MHz}$ cavity conditioning stand, and the $850-\mathrm{MHz}$ amplifiers for the beam line.

The 425-MHz tetrodes are 300-kW devices that supply power to the radiofrequency quadrupole (RFQ) and to the two intermediate matching sections (IMS). AT-5 designed and built four systems with one as a spare. Even though the IMS requires only $50 \mathrm{~kW}$ for each cavity, all the tetrode designs were the same allowing for ease in manufacturing. The tetrodes have worked very well since their installation and have been improved since their conception by adding new Allen-Bradley control systems. This has made interfacing to the overall GTA control system easier.

The $850-\mathrm{MHz}$ conditioning stand is a stand-alone unit with its own power supply and capacitor bank. This system was the original prototype for the $850-\mathrm{MHz}$ amplifiers that will be used on the drift tube linac (DTL) sections of the accelerator. The system is used continuously while we are building the DTL portion of the rf system. Because we have a dual-klystron modulator, we are able to condition cavities in the conditioning vessel while using the other klystron to drive the first DTL tank for experiment $2 \mathrm{~A}$.

The low-level rf design supports both field control and resonance control of the cavities. It also supports many pick-up loops enabling the accelerator physicists to monitor accelerator performance. Additionally, we provided the $\mathrm{rf}$ reference rack that distributes the $425-\mathrm{MHz}$ and $850-\mathrm{MHz}$ reference signal to the various rf and diagnostic components.

The high-power rf system for the 10 DTL tanks for experiment 2D is under construction. This system uses a 5-A high-voltage power supply and a single large capacitor bank that provides power to all 10 klystrons. The integration of the system will be performed in FY 1993. After this system is complete, we will retrofit the conditioning stand to upgrade it to the production design of the DTL systems.

GTA has been a showcase for AT-5 and has allowed us to demonstrate a wide variety of expertise in accelerator if power.

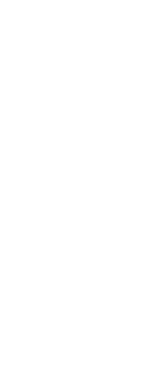

\section{High-Power rf System Development and Production}

The GTA 850-MHz klystron amplifier system has many unique design features. By combining two klystron amplifiers in the same oil tank with a common high-voltage controller, we realize significant economy of hardware and system volume (Fig. 5.1). By using modern high-voltage modulator design techniques, we realize fiber optic control. Additionally a feedback circuit topology minimizes output amplitude droop from the klystron. An advantage of the mechanical layout is that almost all electronic components are located on a removable lid, easing maintenance and ensuring a minimal down time. In the unlikely event that a modulator unit fails, it can be quickly swapped out and repaired off-site.

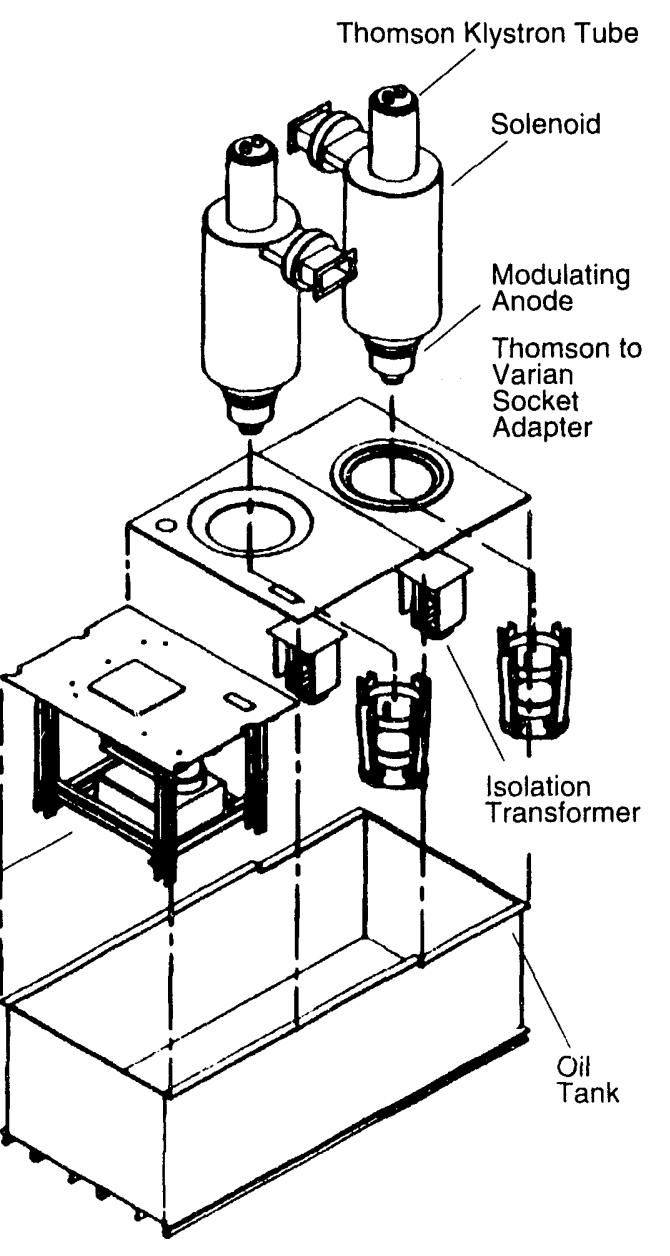

Fig. 5.1. Two-klystron modulator. 
A view of the lirst GTA production modulator being installed is shown in Fig. 5.2. When completed. the of stations will be capable of producing over $12 \mathrm{MW}$ of $\mathrm{rl}$ power with al 2-ms pulse wicith and a 10-Hz repetition ritte.

The control hardware and software for the GTA klystron systems have been optimized from the engineering prototype to provide more offective system interface and control. Each klystron stand has its own supervisory controller for equipment monitoring and sequencing. Unlike the previous standalone rf stations, equipment parameters and stattus must be transmitted (o) the GTA master control room. Each station must be ready before the accelerator can operate. An example of an improved local control screen is shown in Fig. 5.3.

We designed the klystron capacitor bank"s energy-storage system with personnel safely as the utmost concern. Consequently, redundant discharge patths and charge-state monitors are included. To increase personnel salety, the GTA energy storage system includes ergonomic considerations and advanced proection electronics. The capacitor bank uses interlocked and hanging ground hooks in the calpacitor vault entry doorway requiring the operator to physically remove the salfety hooks before entry into the vault is possible. An electronic ground liaule interrupter (GFl), a new implementation for this type of hardwate, effectively protects personnel and equipment from lethal shocks. The system is interlocked such that if the cill malfunctions or is nonoperational, the power system will shut down safely and inhibit further operation. Allentive engineering has also yielded a clean. coronatree system design.

$A$ view of the 5()() -k.l cappacitor hatnk system is shown in Fig. 5.4.

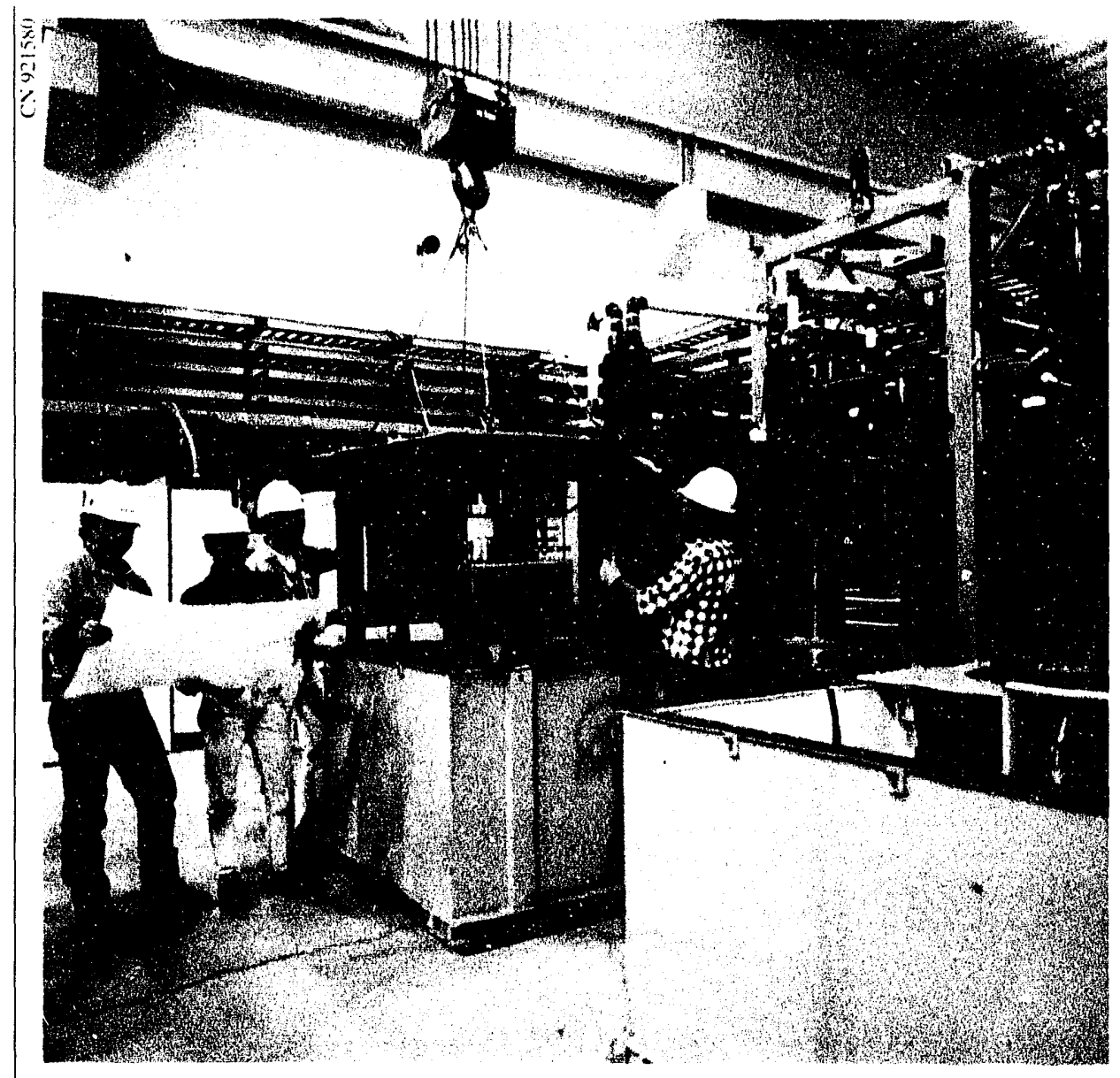

Fig. 5.2. Installation of first production modulator (left to right: Bill Reass. Bill Norm, lohin Buncrofit. Phil (ritelli).

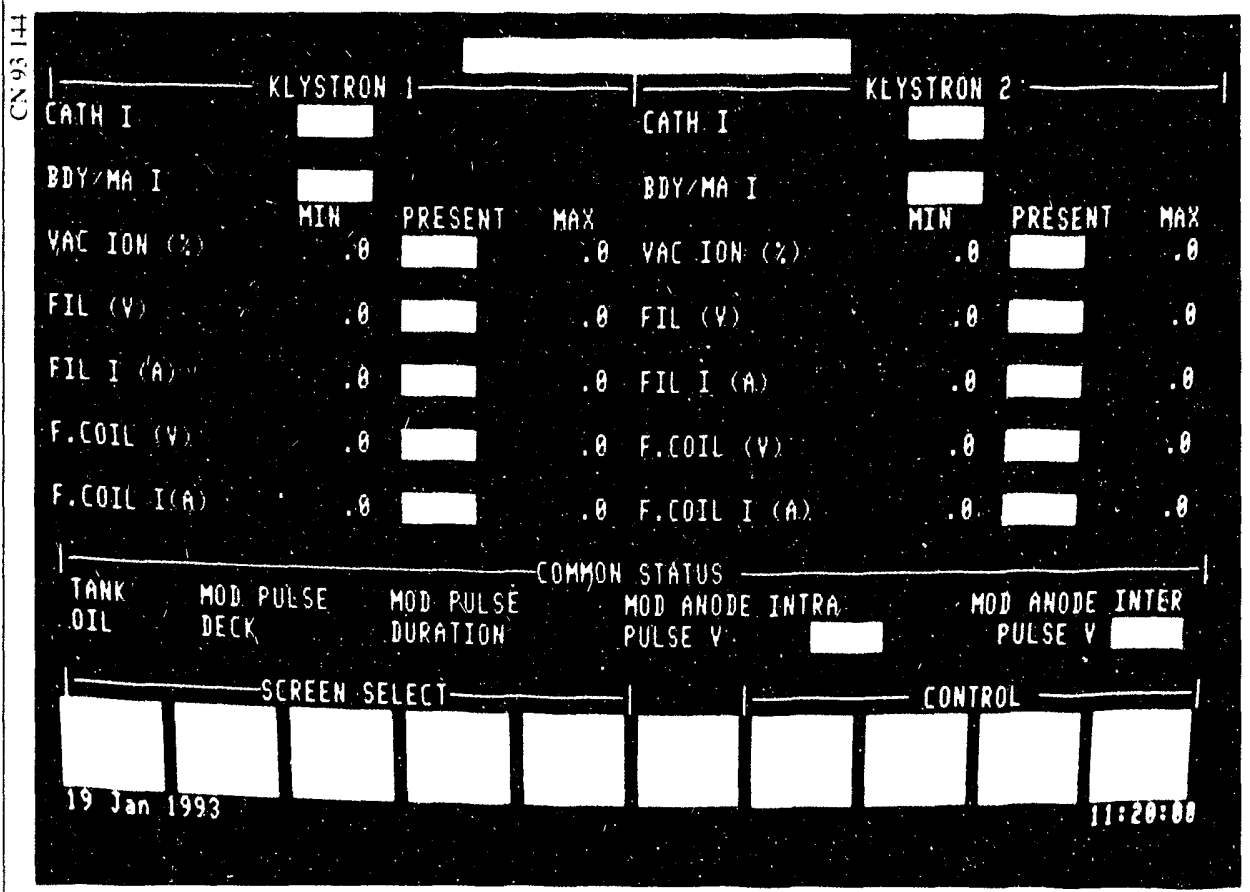

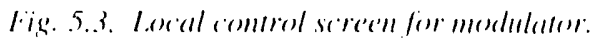




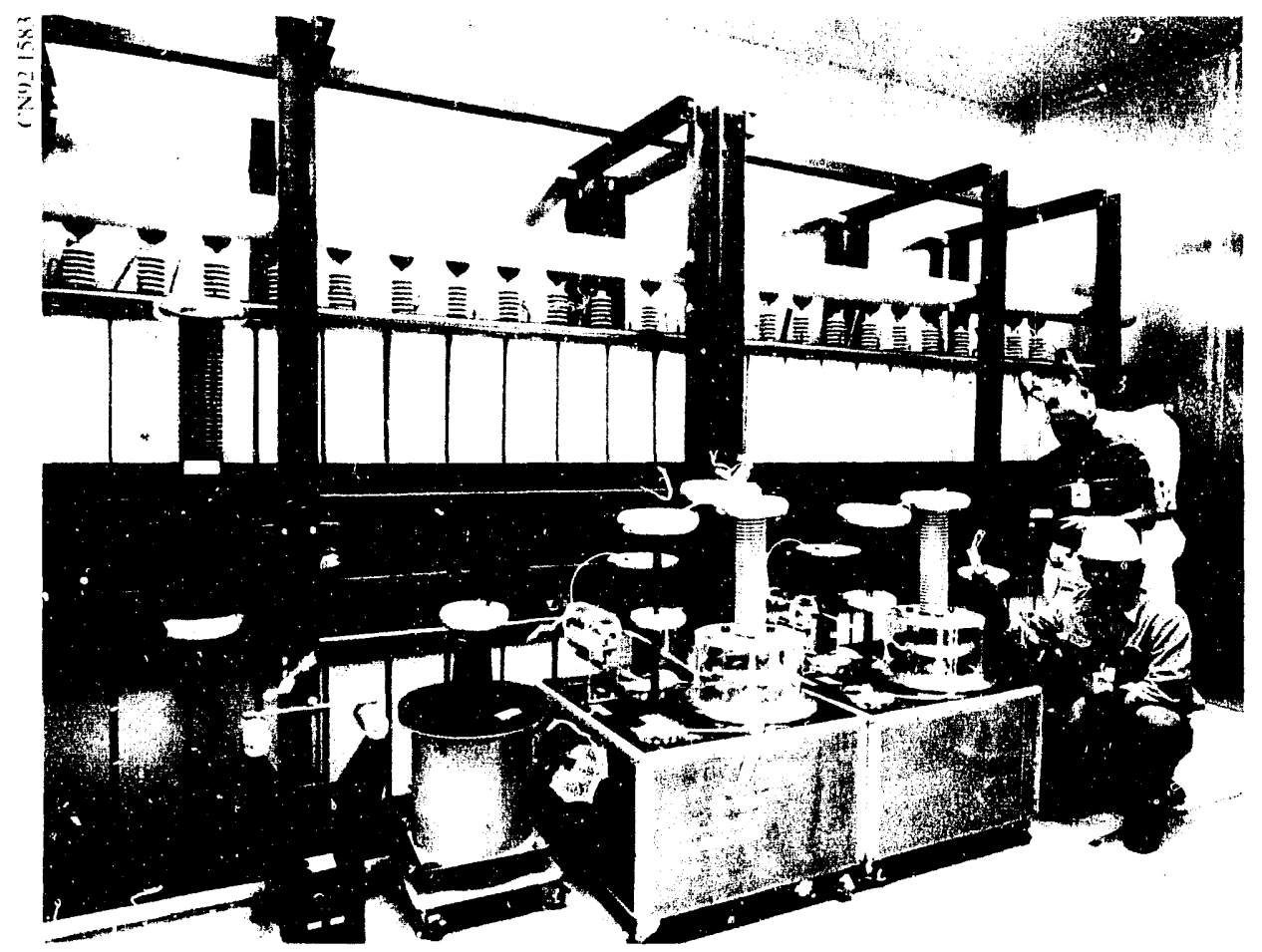

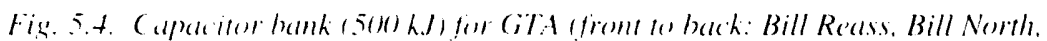
John Bancrolth.

\section{RF System Integration with GTA}

In FY 1992, substantial progress was made in integrating the GTA rl system. In April 1992. the rf systems team was formed to improve the reliability, availability, and operability of existing equipment for experiment $2 \mathrm{~A}$; 10 prepare for expansion to support experiment 2D: and to bring new if components on line. The final fabrication stages of the of klystron stations for the DTL are shown in Fig. 5.5.

Standard system engineering techniques were applied. albeit informally, to integrate the GTA rf system. Major hardware components were taken off line and run through an intermediate hardware and sof tware integration and verification process. Several nagging reliability problems were attended to. Operator interface screens used for controls gain scheduling were rede-

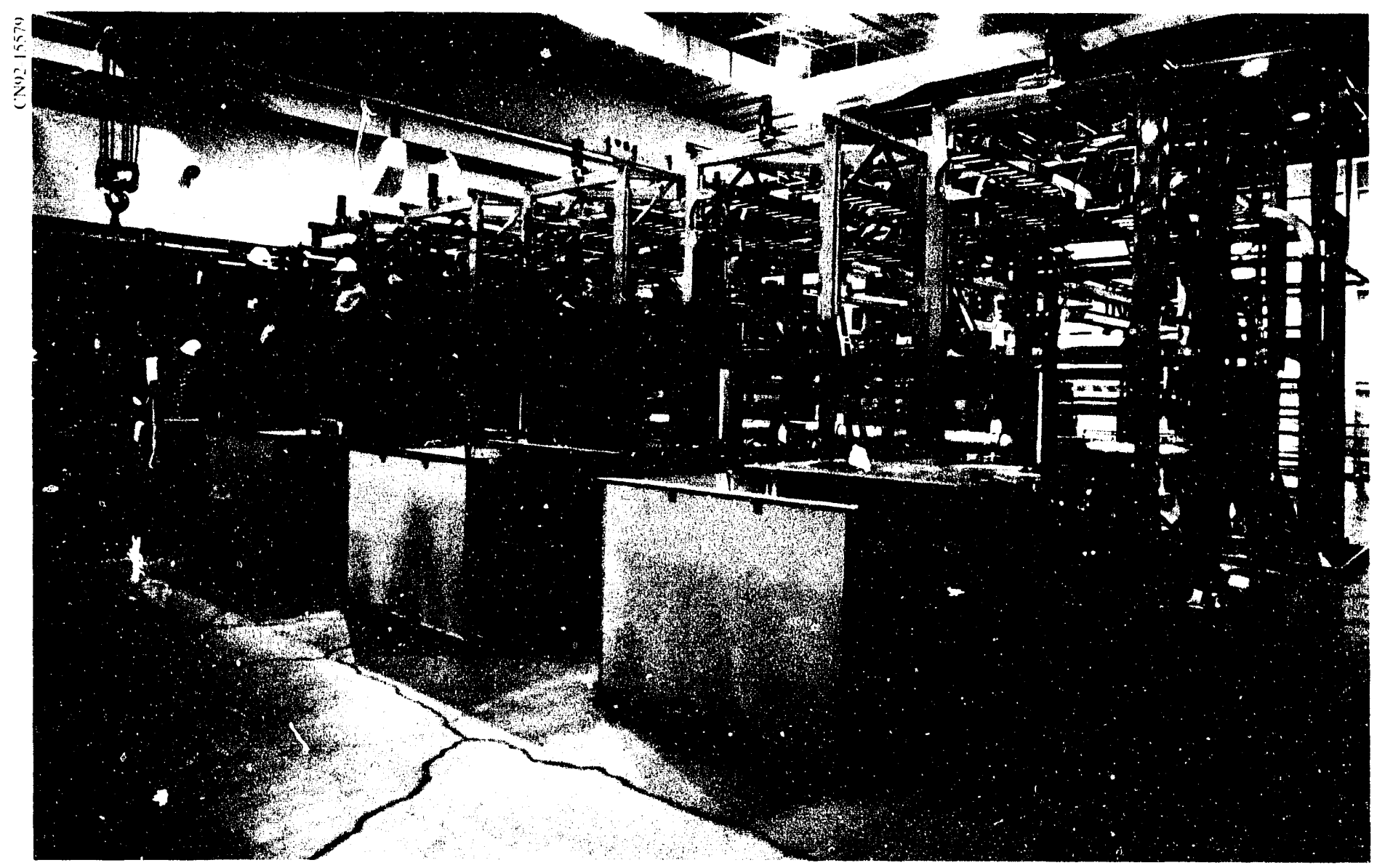


signed for easier use and increased fault tolerance. All system components were then reintegrated, along with their respective software, and the entire if system was put through a verification procedure. As a result, the typical of turn-on time for operations was redaced from 4 hours to under $1 / 2$ hour. Also, rf operations have been essentially uninterrupted during beam experiments, with minimal operator interaction. The acquisition rate of quality experimental data during experiments is at an unprecedented high level due, in part, to these improvements in availability, reliability, and operability of the of system.

Several activities are under way to ensure continued success in the GTA rf sector. We are developing a formal product-oriented work breakdown structure and will negotiate ari integrated requirements document and all required interface control documents based on this work breakdown structure. Formal verification processes will be instituted to track compliance to requirements. These efforts will further improve the design quality and implementation processes.

\section{Automated Klystron Test Stand}

The automated klystron test stand was designed and fabricated in FY 1992 to verify the manufacturer's performance data before the amplifiers are commissioned in the GTA. To monitor and record the status of a klystron, this test stand uses a LABVIEW-based data acquisition and control program (Fig. 5.6). The LABVIEW system interfaces with the Allen-Bradley PLC5/15 con- troller that monitors and records the status of the power supply, transmitter, and other safety interlocks. Thus,

LABVIEW automatically records the operating voltages and currents of each klystron as the lube is being tested.

Three automated tests are performed to characterize the tube performance: swept frequency, power transfer, and a Rieke diagram. An example of swept frequency results is shown in Fig. 5.7. Ten out of the twelve Thomson klystrons were tested this year; test results are compiled in AT-5 memoranda.

\section{RF Controls}

In FY 1992, improvements in the Downconverter (DCM), Vector Detector (VDM), and Upconverter (UPM) modules allowed the design of the core LLRF system to be frozen. In Febru-

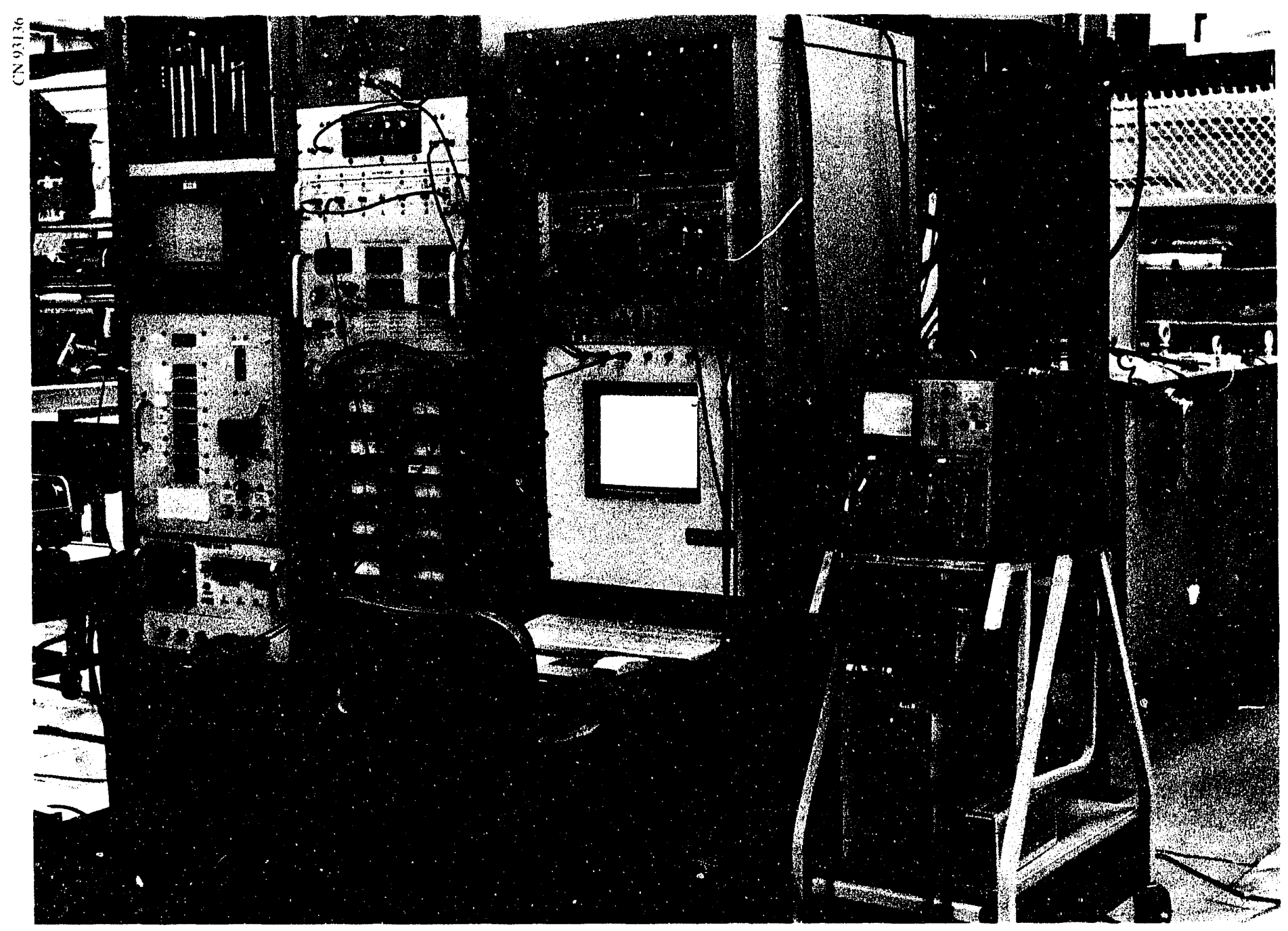

Fig. 5.6. Alutomated klystron test hardware. 
ary, the three $425-\mathrm{MHz}$ LLRF systems and the first $850-\mathrm{MHz}$ system were integrated into the GTA. The LLRF system for the RFQ was upgraded to include module improvements to allow for a reduction in size. As a result. operational problems in the original configuration were corrected, and the LLRF system for the RFQ was reduced from three to two chassis. The two new LLRF systems for the IMS cavities were also integrated as two-chassis systems. We successfully integrated the new 850-MHz system for the DTL \# 1 cavity and demonstrated its proper operation. To support the $850-\mathrm{MHz}$. system, we upgraded the of reference rack to include $850-\mathrm{MHz}$ as well as $425-\mathrm{MHz}$ outputs.

To deliver this hardware as tested and operational, certain process advances were made. The Automated Test Lab was developed and constructed during 1992. The test lab includes the test console, test data storage. tested components, and instrument manuals as well as work space for component troubleshooting. The test console, shown in Fig. 5.8, contains a UNIXbased $\%$ orkstation capable of remotely controlling all the rack-mounted instruments and switching modules. All test software is written in Hewlett

Packard's graphical programming language called VEE. We first demonstrated the facility in late 1992. A GTA UPM was completely tested with the facility. The console, testing software, testing fixtures, and cabling harnesses all had to be completed. During the testing, we reconfigured instruments, actuated switches, and recorded measurements in a totally automated manner for the complete module test.

We performed system testing on all five of the systems to verify the LLRF subsystem integration process before delivery to the GTA. In addition, we created a definite customer and acceptance test to enable system verification upon delivery to the GTA rf team. Lastly, operator manuals for all the modules were completed to facilitate understanding and troubleshooting.
Frequency sweep klystron $\# 8, I C=28.3$ A, $1 / 5 / 93$

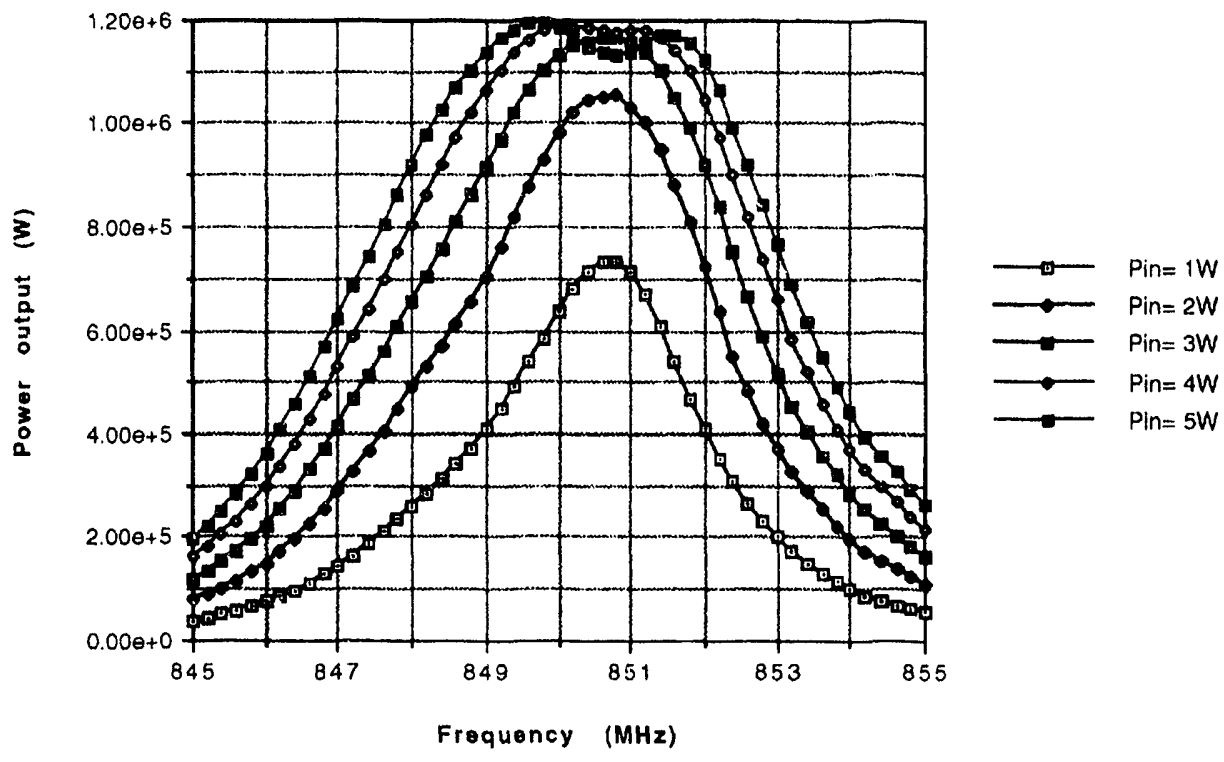

Fig. 5.7. Sample of automated klystron test results.

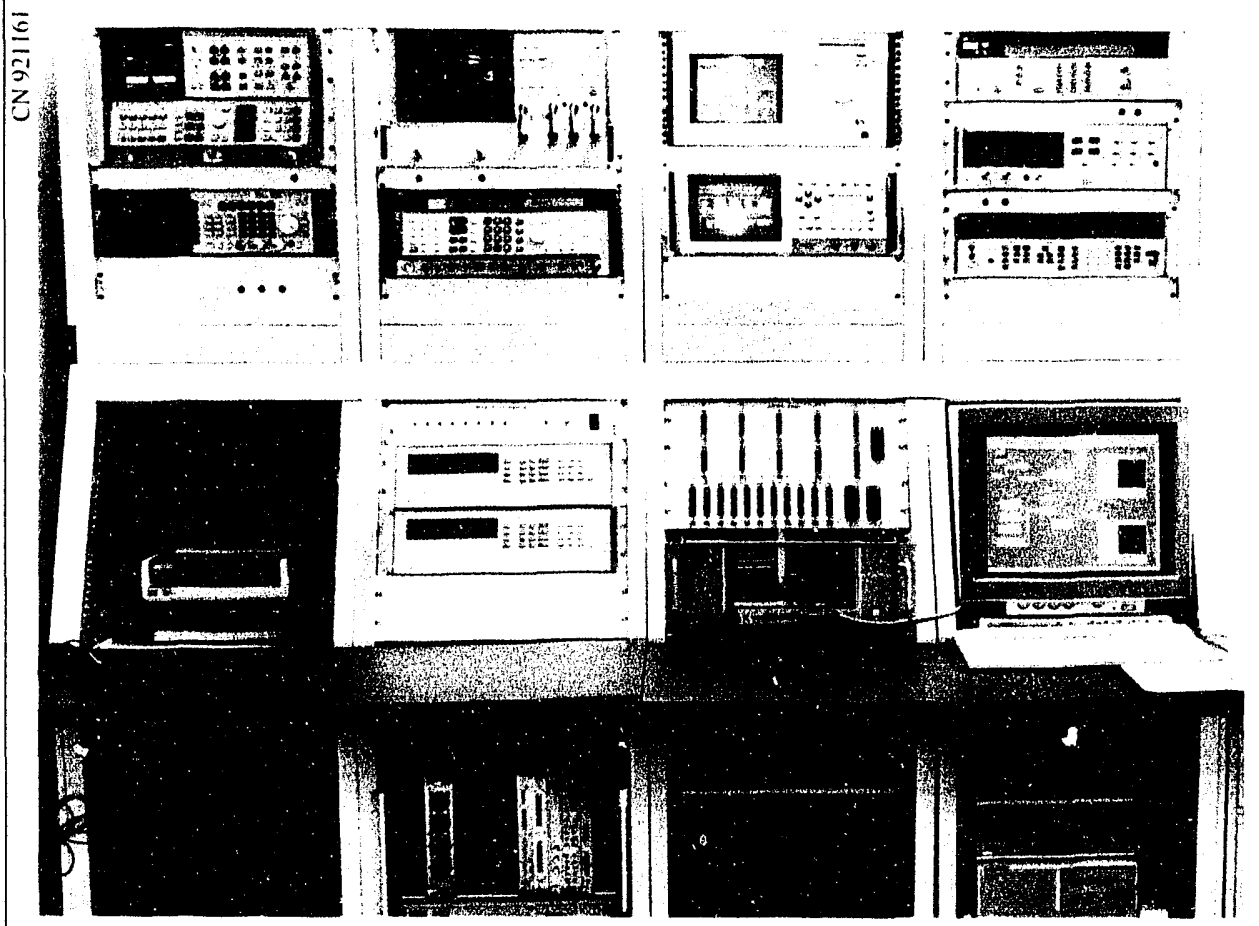

Fig. 5.8. Radio-frequency (rf) control module test lab. 


\section{Feedforward Module}

The Adaptive Feedforward Module, which wats developed for the University of Twente, was lested in an experiment on the GTA. The feedforward topology is shown in Fig. 5.9. This feedforward module demonstrated the use of adaptive feedforward technology and provided a real method to extend the sys$1 \mathrm{em}$ bandwidth beyond the closed-loop control limit. Beam transient disturbances were nearly eliminated, an impossibility with the existing system. Figure 5.10 shows one of the error signals before and after feedforward is applied. The beam turn-on transient wats almost completely eliminated with the feedforward. Additional implementation of the feedforward device on the GTA showed it to be a useful replacement for the current system. The theory may be used in the next generation of of control equipment.

\section{University of Twente}

Los Alamos Nattional Laboratory (I.ANI.) entered into a collaboration with the University of Twente in the Netherlands to build a free-electron laser (FELL) for research applicaltions. AT -5 was tasked by the University of Twente to build the rf controls, the if driver, and the system interlock controls. AT-1 and AT-7 were tasked to provide other elements of the aceelerator. (Figure 5.11 shows hardware installed in the University of Twente accelerator valult.) Based on previous hardware developed for the GTA, AT-5 developed new designs for the control software (based on LABVIEW commercial software) and for the klystron driver becalise of the specific freguency (130) $\mathrm{MHz}$ ) and power requirements (approximately $\mid \mathrm{kW}$ ).

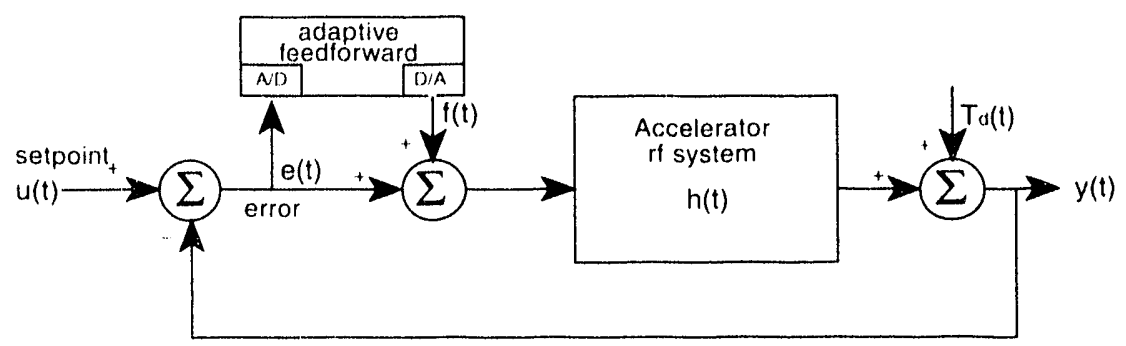

Fig. 5.9. Fendforward system topologey:
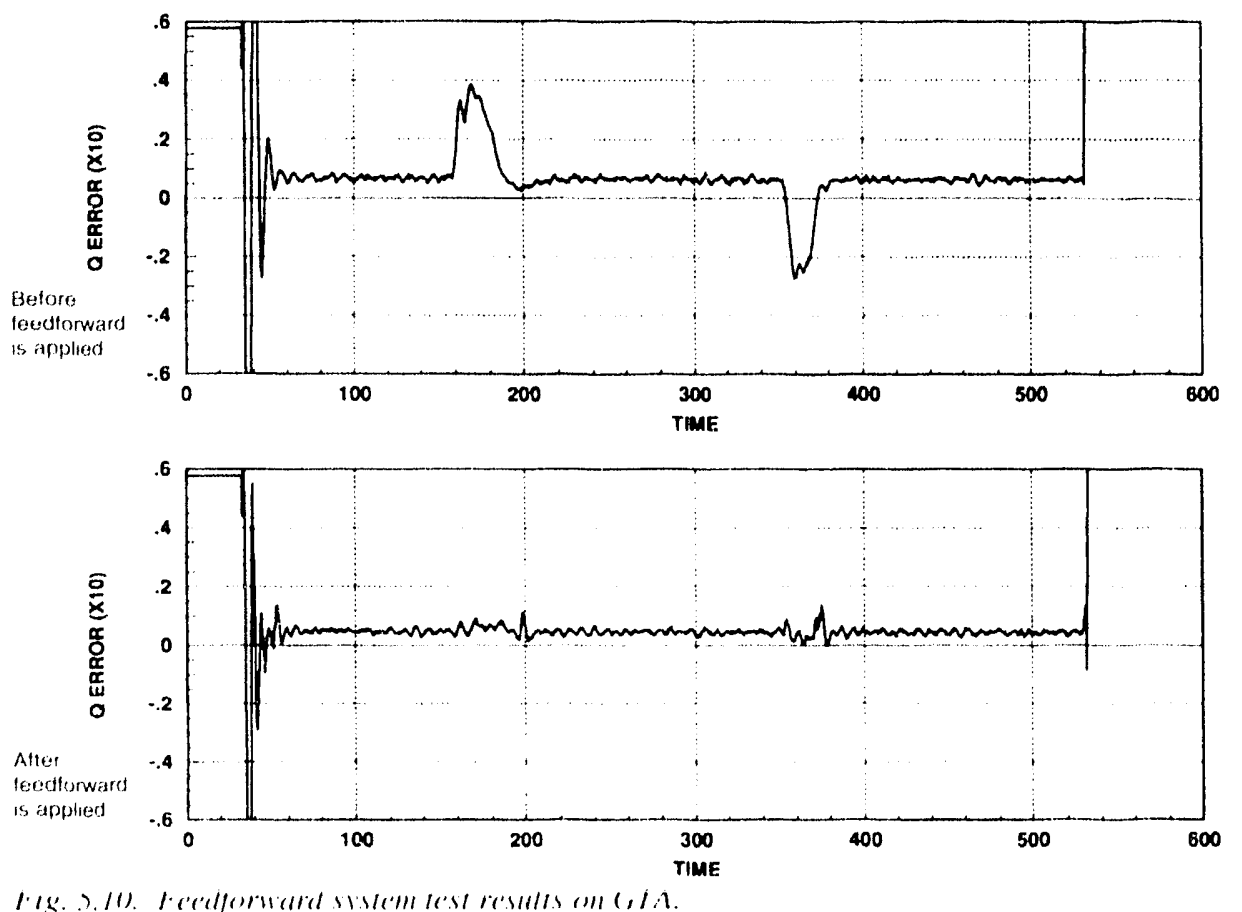

The high-power radio-frequency system for the University of Twente provided by AT-5 consists of a 130)-MHz preamplifier providing in excess of $1000 \mathrm{~W}$ of drive power (0.02\% duty fictor, 2(1)- $\mu$ s pulse width) to the klystron; a waveguide run between the klystron and the accelerating structure; and a control terminal to monitor and control the high-voltage system. The klystron driver is a four-stage amplifier with three solid-state stages and one planar-triode calvity-amplifier stage. The monitoring and control system consists of an Allen-Bradley Industrial Controller and a T30 terminal, which provides the user interface. In May 1992, AT-5 delivered and installed this equipment and it is operating without problems. This hardware is shown in the left rack in Fig. 5.11.

The LLRF system developed for the University of Twente provides field control, resonance detection, accelerator timing, diagnostic monitoring, and alarm and limit monitoring for their FEL, accelerator. This LLRF system was built into a single rack and included all LLRF equipment, an embedded Macintosh computer, a keyboard, a monitor screen, and all the necessary power conditioning. We also developed a LABVIEW software system for this system that runs on the embedded Macintosh computer and is stored on the computer's hard disk. Consequently, the university was provided with a complete, stand-alone LLRF control system. This hardware is shown in the middle rack in Fig. 5.11.

The LLRF system was completely tested and integrated at LANL to verify its operation before shipment. In addition, this system wals used on the 130)()MH\% AFEL system at LANL and performed suceessfully. The system was shipped to the Netherlands in August 1992. The university was provided with a complete set of documentation, including a system definition and user's manuals for all devices in the system. The universily personnel used these documents, along with our alssistance 
via electronic mail, to perform much of the integration of the LLRF system with their accelerator. As a final training step, an AT -5 engineer traveled to the University of Twente to help with the final integration and to train personnel to operate the LLRF system. Currently, the initial results are favorable, although full system operation will be performed in FY 1993.

\section{Boeing APLE}

In 1992, we delivered a VXIbus-based LLRF system to provide rf field control, resonance control, timing, and diagnostic monitoring for the first four 433.33-MHz cavities of the Boeing Average Power Laser Experiment (APLE). We installed this system at Boeing in February 1992, and it was used successfully as part of their highduty experiments that summer. Furthermore, we provided on-site training at Boeing to enable their personnel to understand, calibrate, and operate the LLRF system.

In addition to the delivered system, we negotiated a contract to deliver Boeing another LLRF system to accommodate the remainder of the FEL project. The new system will include another 433.33-MHz control system similar to the existing one, a new rf reference system, and a phase-stabilized transport system.

\section{Argonne Advanced Photon Source (APS)}

Argonne is building a new facility called the Advanced Photon Source (APS). AT-5 negotiated a design contract with Argonne to modify some of our existing rf control equipment to operate at $2856 \mathrm{MHz}$ with a higher measurement bandwidth. This included design modifications for the

Downconverter, Envelope Detector, and Vector Detector modules. The design work has proceeded well, with Argonne receiving and testing the engineering prototypes. Presently, the final designs are $50 \%$ complete and should he finished early in 1993. Argonne requires many (40-80) of these modules for the APS project in 1993; therefore,

LANL will be heavily involved in providing Argonne with these modules.

\section{Superconducting Super Collider (SSC) Laboratory Linac}

AT-5 has been working with the Superconducting Super Collider (SSC) Labo- ratory on components for the linac for several years. The primary tasks have been to develop and fabricate a 427-MHz tetrode-based amplifier for test stand operation at the SSC and to develop and fabricate low-level if controls. In addition to providing hardware, AT-5 has collaborated with engineers at the SSC on their system design, particularly the design of the feedback control system.

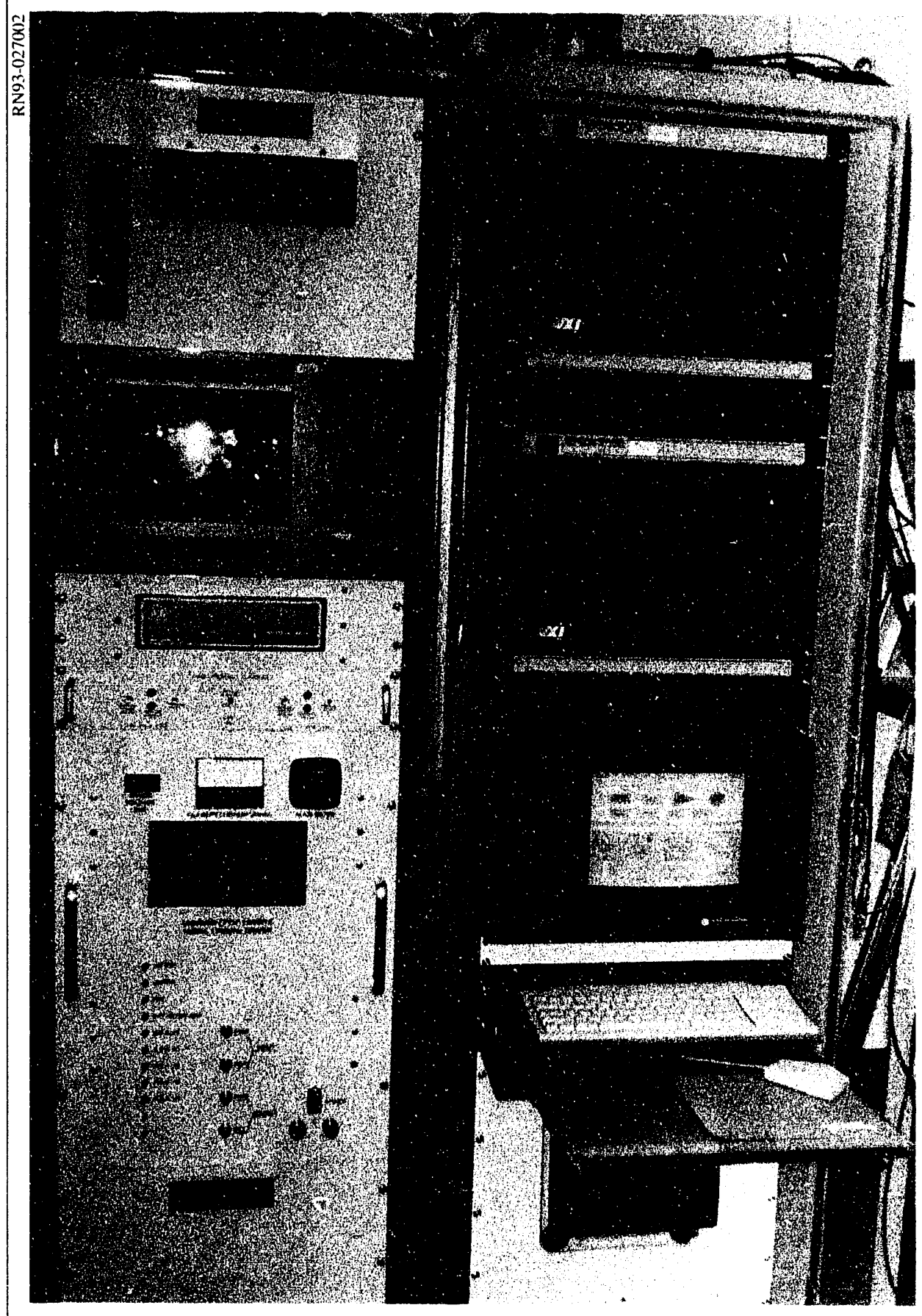

Fig. 5.1\%. Unversty of livente hardware installed in ace elerator area. 
The first control system was delivered (1) the SSC in early FY 1992. In addition. we loaned the SSC a cavity/amplifier simulator that allowed them to use the controls, apply disturbances, and measure responses. The simulator results were used by the controls personnel at the SSC to justify further development and fabrication effort. In the last half of FY 1992, almost all modules needed for two more complete control systems were provided to the SSC: in addition, the SSC returned the cavity/amplifier simulator tafter building a simulator of their ow'n).

SSC and LANL collaborated to modify the design of the +25-MHz tetrode amplifier developed for the GTA to meet a 427-MHz requirement for the SSC. The GTA amplifier is a 30()$-\mathrm{kW}$ pulsed amplifier with a maximum pulse width of 2 ms and a pulse repetition frequency of $10 \mathrm{H} \%$. This design was modified to meet a 6()()$-k W$ pulsed requirement for the SSC with a pulse width of $1(0) \mu$ s and a pulse repetition frequency of $10 \mathrm{H} \%$. Fabrication was completed at LANL, and the amplitier was delivered to SSC and tested in May 1992. The amplifier design included a new lower-loss input cavity for the final amplifier and an upgrade in the
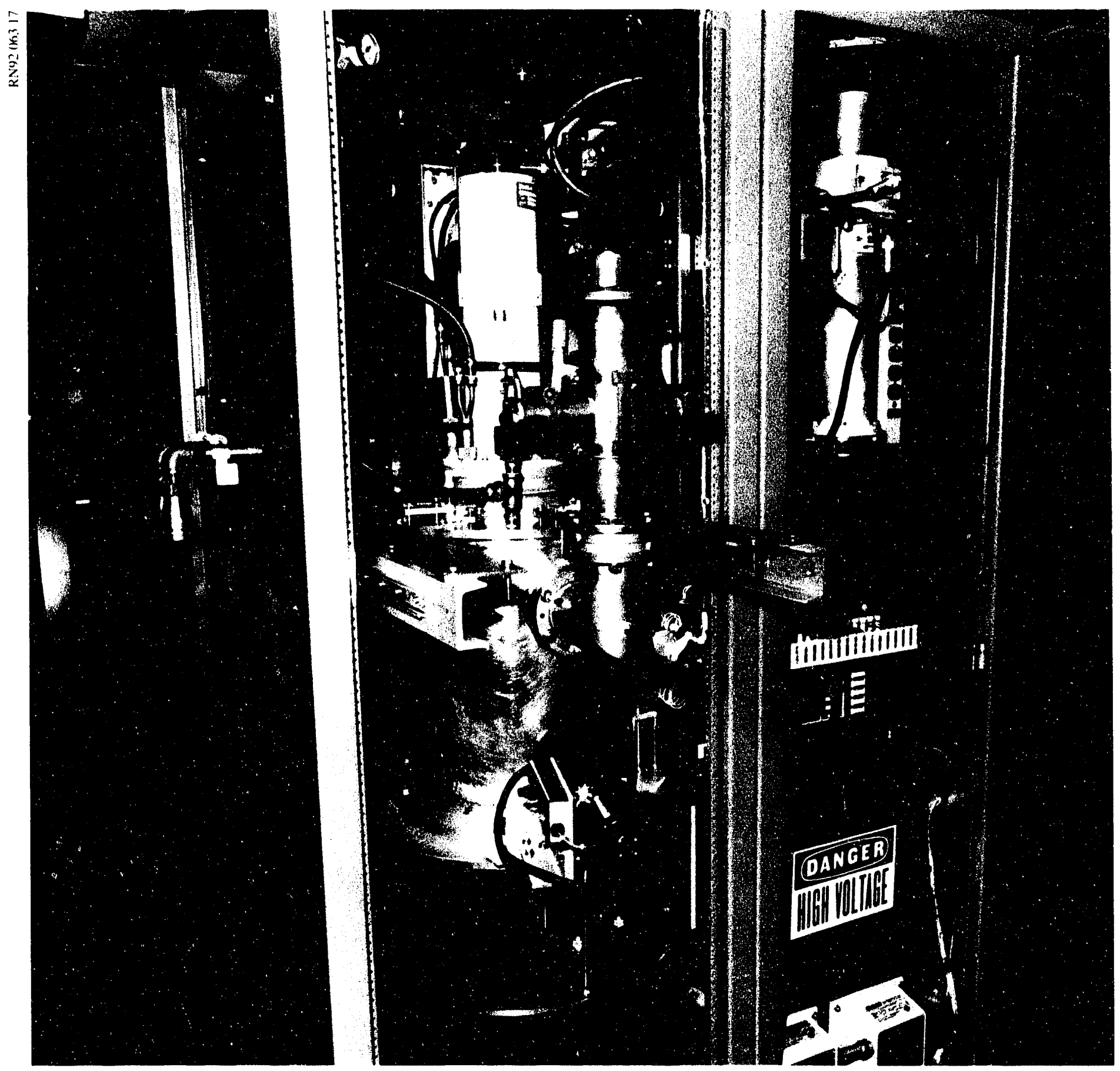

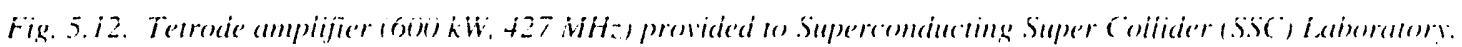




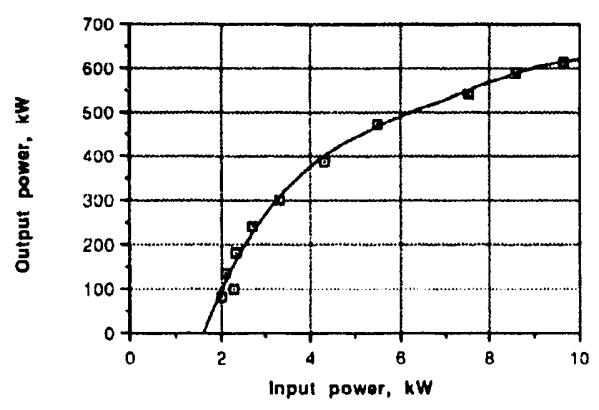

Fig. 5.13. Power transfer curve for SSC Laboratory tetrode amplifier.

amplifier computer controls from the Pushbutton sheet-metal panel to a programmable terminal. In addition, the high-voltage power supply design for the final amplifier was drastically improved over the GTA design and has become our current baseline for this class of amplifier. The completed SSC amplifier is shown in Fig. 5.12 and a sample of the test results is shown in Fig. 5.13.

AT -5 has been involved with the SSC in designing and developing the lowenergy booster (LEB) cavity since the beginning of the program at SSC. The AT-5 participation resulted from our experience with perpendicularly biased, ferrite-tuned cavities. This experience was gained during the Advanced Hadron Facility study at LANL.

The basic configuration for the SSC LEB cavity remains as originally proposed by AT -5 . This configuration is shown in Fig. 5.14. The cavity is a quarter-wave resonant structure, with the accelerating gap at the high-voltage end and the ferrite torroids at the highcurrent end. Wave propagation is in the coaxial mode throughout most of the structure up to the ferrite region, where the propagation is in the radial direction. The control bias $\mathrm{H}$ field direction is across the torroids in the longitudinal direction. The circuit's high-power excitation is provided by a $4 \mathrm{cw} 150.0(0)$ E tetrode tighty coupled to the cavity through a coupling capacitor located between the accelerating gap and the ferrite tuner.

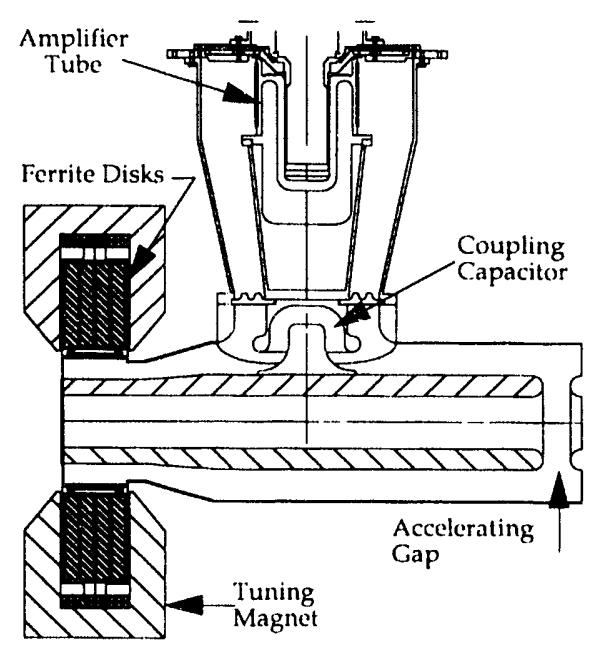

Fig. 5.14. Low-energy booster (LEB) cavity configuration for SSC.
Two different ferrite cooling techniques are being pursued by the SSC. The liquid cooling technique proposed by AT- 5 is being developed at the SSC, while an alternate technique, using berylium oxide disks glued to the ferrites, is being developed in Siberia by the Institute for Nuclear Physics Laboratory for the SSC. Tests at the SSC during the past year have validated water as a suitable ferrite cooling fluid within the rf structure.

In collaboration with the SSC power supply group, AT-5 modeled the magnet string in the LEB to characterize the magnet system's electrical design for the $L E B$, the first of five rings that make up the SSC. The LEB, which consists of 12 nearly equivalent magnet cells arranged in a 57()-m ring, is designed to accelerate protons from 600 $\mathrm{MeV}$ to $12 \mathrm{Gev}$, and it can function in one of two modes (Fig. 5.15).

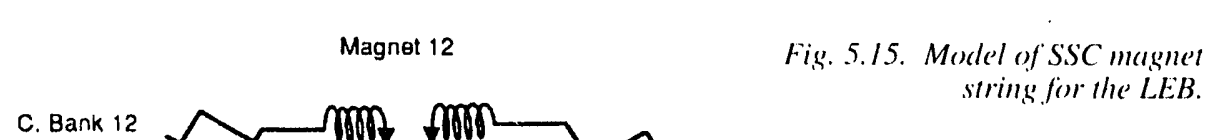
string for the LEB. 
The primary operating mode is as a 10$\mathrm{Hz}$ rapid-cycling proton synchrotron. In this mode, capacitor banks are used to make the entire magnet circuit resonant at 10 Hz. (Fig. 5.16). However, because capacitor performance is alfected by temperature change and the enclosure in which the capacitors are to be installed is unheated, total capacitance in the system is expected to vary as the daily ambient temperature varies. Therefore, to maintain resonance at 10 Hz. each capacitor bank includes additional trimming capacitors that may be remotely switched in and out of the circuit. This resonant system eliminates the requirement of otherwise having to provide (and recover) a large amount of reactive power.

Because the acceleration cycle is from $600 \mathrm{MeV}$ to $12 \mathrm{GeV}$, the magnet current must swing a factor of 10 , from 400) A 10 400() A. Because capacitors cannot conduct direct current (dc), the capacitors are placed in parallel with inductors (chokes) so that the magnet system can operatc with a biased sinewave excitation. The primary focus of the modeling effort is to analyze the electrical propertics of this magnet power system.

The second operating mode of the L.EB is to cycle at $1 \mathrm{H} \%$ as a ramped proton synchrotron, with flat $(\mathrm{dl} / \mathrm{dt}=0)$ " firont porches" and "lops" for injection and extraction (Fig. 5.17). This 1-H\% mode is accomplished by driving the magnet system with silicon controlled rectifier (SCR) power supplies using predetermined waveforms.

We analyzed both the 1()-H\%-resonant and the 1-Hz-ramp modes using as the primary analysis tool SPICE, version 3E. which operates on SUN workstations.

\section{Directed Energy Weapons Power Integration (DEWPOINT)}

The goals of the Directed Energy Weapons Power Integration (DEWPOINT) program are to design and fabricate a lightweight, stateof -the-atr, space traceable, 5()()-kW cw rf amplitier. GTE is designing and fabricating at $1-k W$ solid-state hybrid amplifier module (HAM) composed of solid-state triodes (SST) and a microchannel heatsink provided by Lawrence Livermore National Laboratory. The specifications on the HAM are output power of 1 $\mathrm{kW}$ cw, efficiency greater than $65 \%$, and a gain greater than $9 \mathrm{~dB}$. In FY

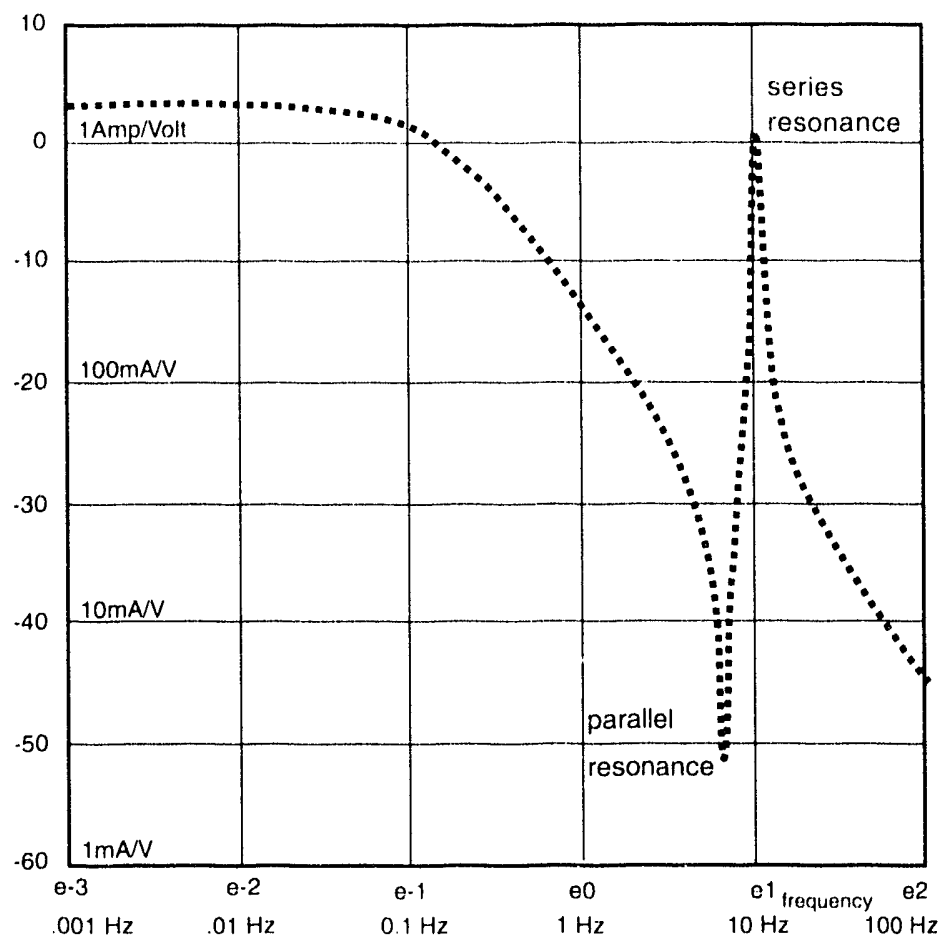
1993, G'TE will

test a submodule of the HAM that consists of four SSTS and will produce greater than 250-W cw. Upon completion of these lests. GTE will begin fabricating the required number of HAMs to huild a $25-\mathrm{kW}$ amplifier. Westinghouse (WEC) is responsible for designing a $5(0)-k W$ splid-state amplifier using the HAMs as fundamental building blocks. After completing this design, WEC will fabricate a $25-\mathrm{kW}$ cw demonstrator that is derived from the 5()()$-k W$ space-traceable amplifier. The prime power for the amplifier will be designed by Electromesh Institute in Russia. Currently, the institute is designing a hyperconducting alternator to our specification.

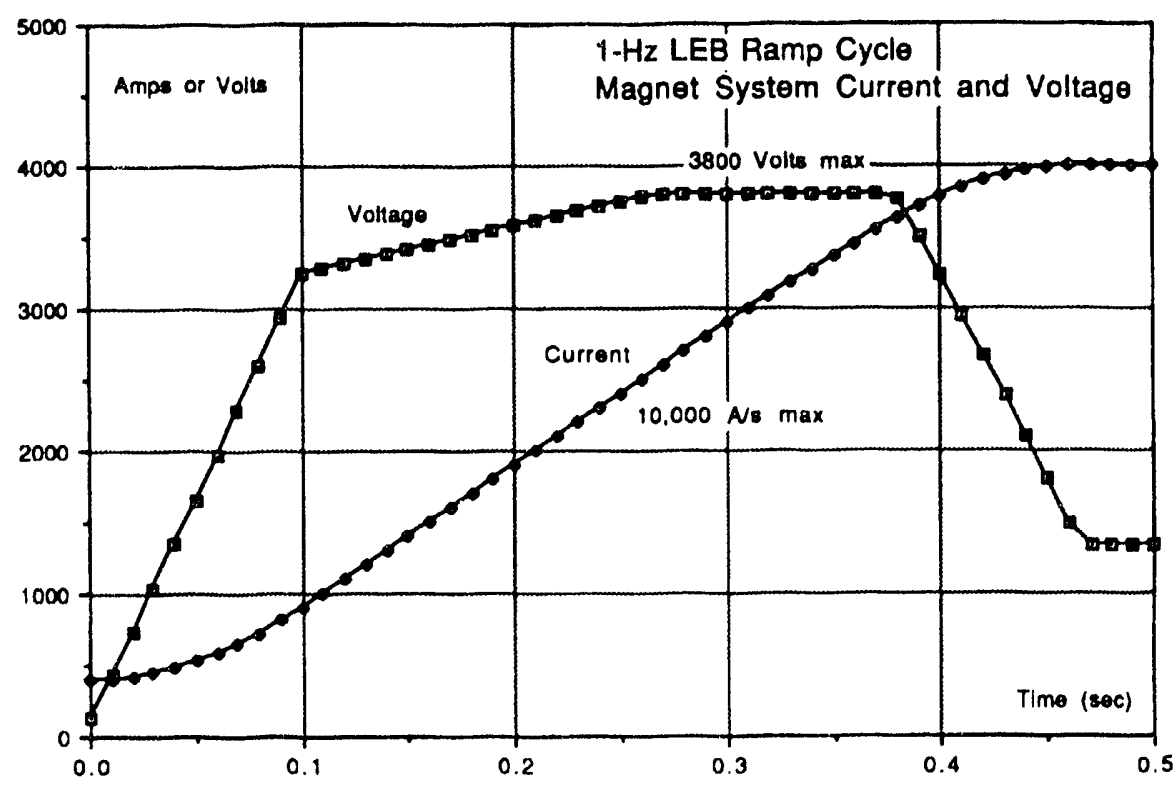

fïe. 5. 17. Masenet modeling results shewing the 1-He operating mole. 


\section{Plasma Source Ion Implantation}

The plasma source ion implantation (PSII) process is a new manufacturing technology being codeveloped by AT Division, $\mathrm{P}$ Division, $\mathrm{X}$ Division, and MST Division in collaboration with General Motors Corporation and the University of Wisconsin. The intent of this Cooperative Research and Development Agreement (CRADA) is to develop the PSII process on a large scale for the automotive industry. PSII is a process to tribologically alter material surfaces by immersing an object in a plasma and then attracting the ions to the object with a high negative voltage. Tribology is the study of the science of friction, wear, and corrosion. For automotive applications, these objects may be drive-train components, machine tooling, or stamping dies. As an example of the process, a metallic part might be immersed in a nitrogen plasma and pulsed at high voltage to form a nitride surface layer. The PSII process shows promise as a replacement for the wet chemical bath (plating) process, thereby eliminating the associated hazardous waste.

The AT -5 contribution is to design and develop the high-power modulator systems suitable for commercializing PSII. It is anticipated that the first production-line prototype PSII systems will require peak switching currents of 200 A or more at the $150-\mathrm{kV}$ levels with a few percent duty factor $(0.5$ to $1 \mathrm{MW}$ average). Further modulator and power system development is required to commercialize full-scale automotive assembly-line processing. These rep-rated systems may operate at kilo-ampere pulse currents at the $500-\mathrm{kV}$ levels. A simplified diagram of the first modulator system is shown in Fig. 5.18. A view of the hardware (Fig. 5.19) shows a striking similarity to the GTA rf modulator. It is important to note that the switching technology used for this system is a direct technology spin-off from the Strategic Defense Initiative Organization and GTA programs.

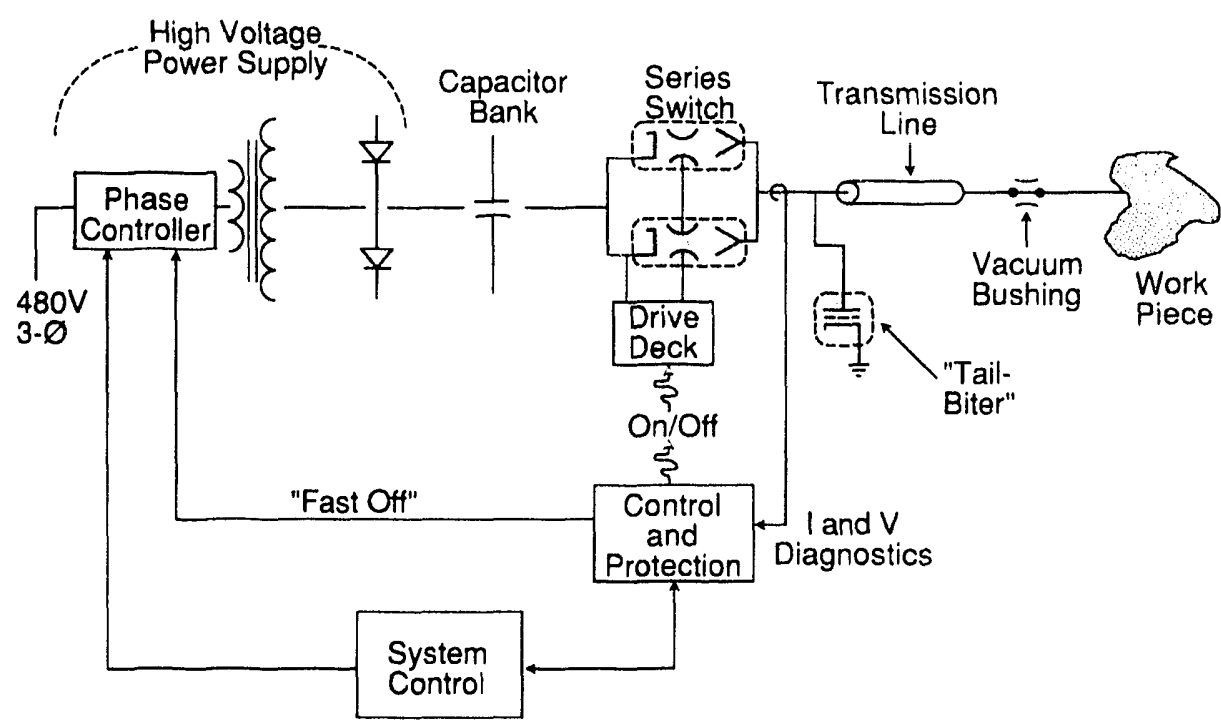

Fig. 5.18. Block diagram of PSIl high-voltage modulator.

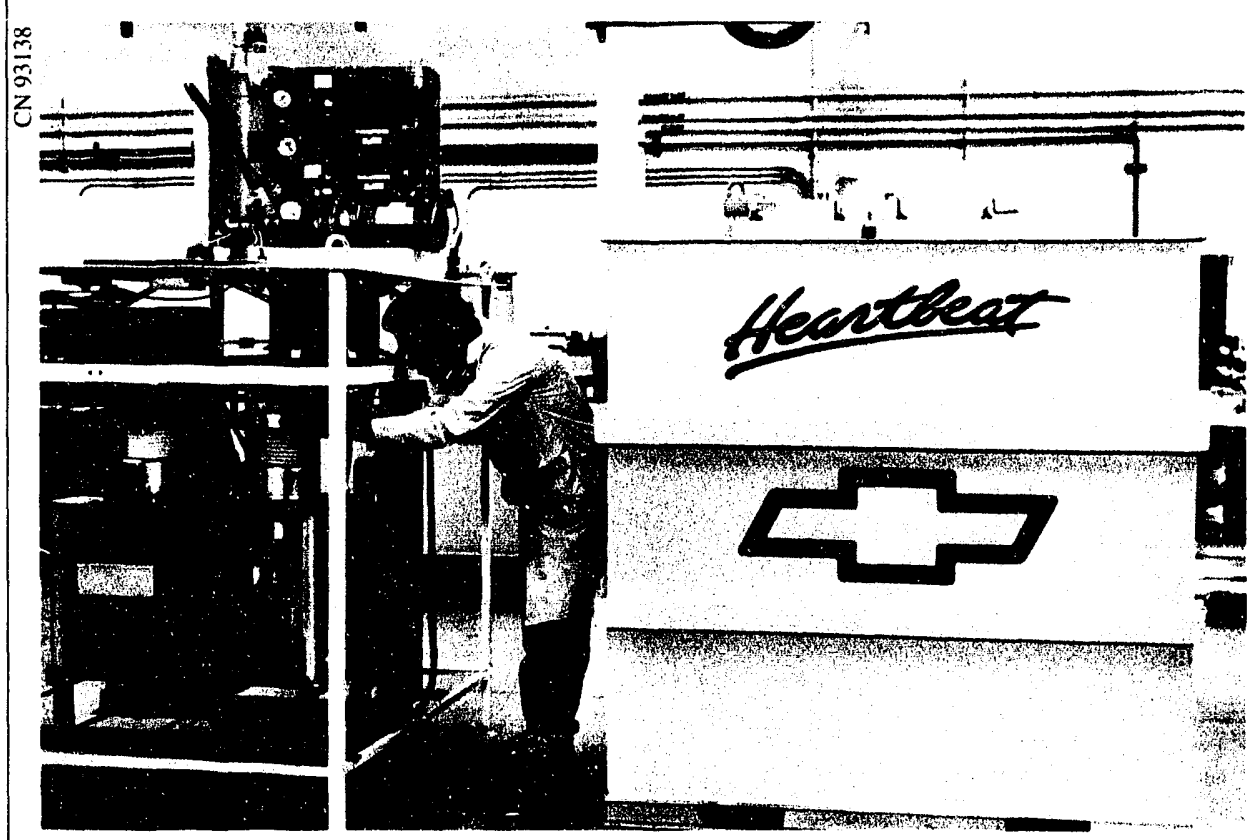

Fig. 5.19. Initial construction of PSII modulator (Ralph Cordova, AT-5). 


\section{Gyrotron}

AT-5 is working with MST-4 to commercialize a Ukrainian technology that involves processing materials with directed millimeter-wave radiation. The effort is a result of a CRADA between LANL and the National Center for Manufacturing Sciences (NCMS). The CRADA involves taking a materialprocessing experimental stand from the Ukraine, rebuilding it to meet US safety standards and quality expectations, and providing an experimental facility where US or Canadian members of NCMS can evaluate applications of the technology. The material processing stand consists of (1) an 84$\mathrm{GH} \approx, 35-\mathrm{kW}$, cw gyrotron with a mode converter to take the overmoded output of the gyrotron and convert it to a concentrated distribution suitable for use in the processing chamber: (2) a superconducting magnet for gyrotron operation; (3) a quasi-optical transport system to duct the gyrotron radiation to a processing chamber; and (4) a processing chamber where the experimental work lakes place. This method of processing is being considered for ceramics bonding, enamel applications, $\mathrm{Si}$ and GaAs wafer processing. polymer hardening, and many other applications. AT -5 has received the gyrotron equipment from the Ukraine and is awaiting funding to refurbish the equipment. The system is expected to be operational 9 months from the receipt of funding. The Ukraine's operational system is shown in Fig. 5.20.

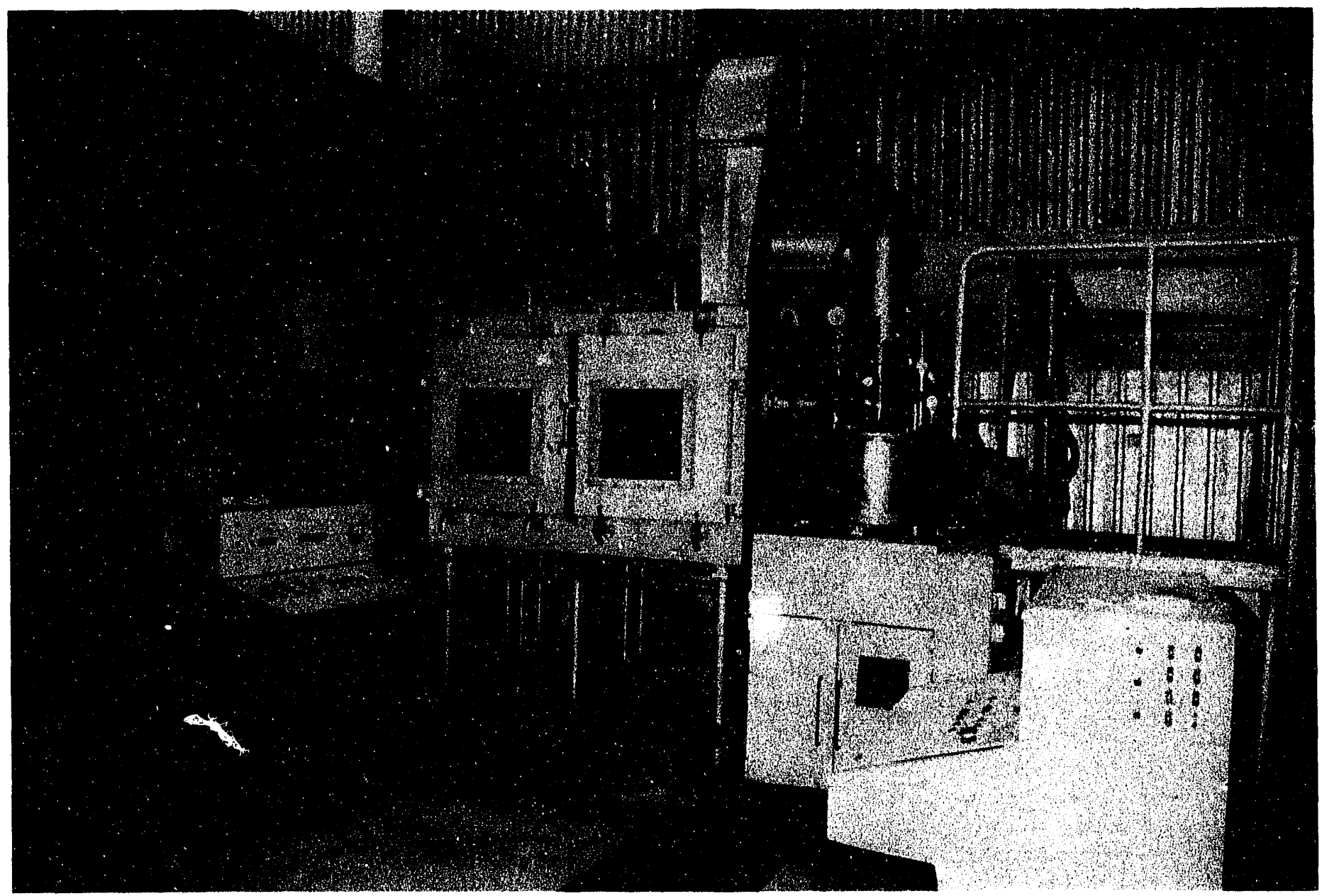

Fig. 5.20. Gyrotron-based materials processing system installed in the Ukraine. 


\section{Accelerator Production of Tritium (APT)}

AT Division has been working for several years on a proposal to produce tritium with a high-energy, cw, proton accelerator. An accelerator-based approach to produce tritium offers environmental and safety advantages over a nuclear reactor. In addition, an accelerator is more easily scaled in both design and operation for reduced levels of tritium production. AT- 5 has been directly involved in this proposal effort because the rf power required is so enormous. The present design requires close to $300 \mathrm{MW}$ of $\mathrm{cw}$ if power. At optimistic efficiencies, this would require more than $500 \mathrm{MW}$ of prime power.

AT-5 has been working on the conceptual design of the Accelerator Production of Tritium (APT) if system, including the rf power generator type, the distribution system, the power conditioning, and component protection. A large part of the effort is based on maximizing the system availability. Studies of existing systems are in progress, including the Clinton $\mathrm{P}$. Anderson Meson Physics Facility (LAMPF), Continuous Beam Accelerator Facility (CEBAF), European Center for Nuclear Research (CERN), Large Electron-Positron Collider (LEP), and others.

Additionally, AT-5 has been working on the APT system design of the beam/ cavity control. High-efficiency operation implies minimal control margin, but minimal control margin implies reduced control response. Tradeoffs must be considered. APT is a very heavily beam-loaded accelerator and will require excellent control to reduce beam losses. Various schemes combining both feedback and feedforward are being considered to meet the control requirements while maximizing operating efficiency.

\section{Accelerator Transmutation of Waste (ATW) and Accelerator Based Conversion (ABC)}

The Accelerator Transmutation of Waste (ATW) and the Accelerator Based Conversion $(A B C)$ of plutonium are additional applications of the highpower proton accelerator technology being considered for APT. Design studies are taking place for all these applications, as funding permits.

The generator of choice for all these applications remains the klystron, but we have learned that the 1-MW CERN klystrons have had several failures, and their life is about 25,000 hours, on average. Reliability concerns also limit the high-frequency power per klystron to $1.0-\mathrm{MW} \mathrm{cw}$. The vendors have improved the $352-\mathrm{MHz}, 1.0-\mathrm{MW}$ klystron to $1.3 \mathrm{MW}$, and they can now make $1.0-\mathrm{MW}$ at $77 \mathrm{kV}$, but this lowers the efficiency to $62 \%$. Thus, at 350 or $400 \mathrm{MHz}$, we can expect klystrons with 1.3-MW output power. However. we have learned that the main cause of downtime is not the klystron, but the cables and connectors, which require considerable attention for maximum system reliability.

Another generator under consideration for these applications is the klystrode because of its improved operating efficiency. The klystrode for the Chalk River Laboratory (CRL) in Canada worked very well and has produced over $70 \%$ dc-to-rf efficiency under a wide variety of conditions (compared to only $68 \%$ for the klystron, and then only under optimum conditions). The CRL klystrode is rated at $250 \mathrm{~kW}$ at $267 \mathrm{MHz}$.
We presented our ideas on the Accelerator Based Conversion ( $A B C$ ) if system to the Russian designers at at Los Alamos meeting. and they presented their ideas based on their use of the regotron (another type of highpower if generator). Although the differences in approach are greal, we have corresponded with our Russian counterparts to better understand each other"s ideas. 

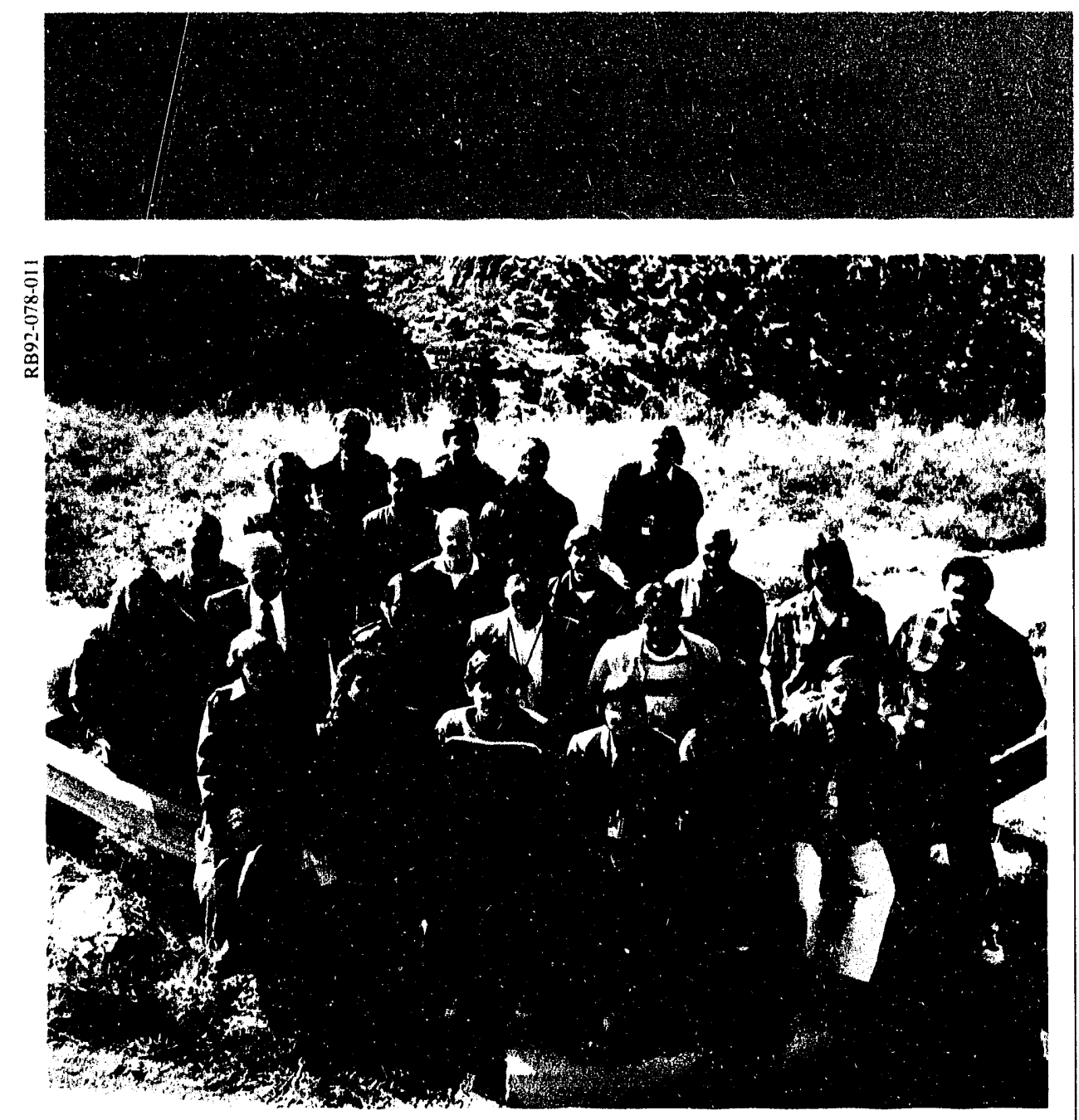

Introductiont

Free-Electrom-Laser Dovelopments.. 75 Al'EX 75

AFEL EXperime'mt. ........................ 7.5

Microwiggler .............................. 7.5

P(OP Experime'nt ....................... 76

Theory and Simulation ..................... 77

Theory...................................... 77

Simulations .................................. 77

Loss Alamos Accelerator

Code Group 80

Sofiware Development and

Maintenance ..............................80

User Consultation .......................80

Distribution of Software and

Documentation 80

Gathering and Dissemination of Information

Other Activities 81 Alpha Simulation Using Neutral Beams

XUV Plasma Source

SSC Collaboration

PSR Development

LANSCE II Proposal

ESNIT Collaboration 82

Accelerator Theory Notes .8 .3

Terhnical Memoranda 8.3 


\section{Introduction}

Group AT-7 provides theoretical and computational support for AT-Division programs and supports free-electron-laser (FEL) activities within ATDivision. We conduct theoretical analysis and develop computational tools applicable to accelerator technology, including activities by the Los Alamos Accelerator Code Group (LAACG) and theoretical research in the accelerator area. AT-7 manages FEL experiments by coordinating work of contributing groups and by providing technical guidance to the FEL program offices for proposals, program directions, and external collaborative arrangements. We engage in extensive research and development on accelerator components and systems pertaining to the enhancement of FEL performance.

\section{Free-Electron-Laser Developments}

\section{APEX}

A Boeing/Los Alamos collaboration is under way to build a high-average power FEL called the Average Power Laser Experiment (APLE). The goal of APLE is to demonstrate that a FEL can produce laser light with an average power of $100 \mathrm{~kW}$. A collaborative effort by many Los Alamos National Laboratory (LANL) groups has resulted in the APLE Prototype EXperiment (APEX). The APEX's purpose is to demonstrate the basic physics and technology of APLE at low-duty factor. The APEX FEL is driven by a $40-\mathrm{MeV}$ electron accelerator that consists of a $1.3-\mathrm{GHz}, 12-$ mode coupled-cavity linac with a 6$\mathrm{MeV}$ photoinjector. The microbunch characteristics include a current of 0$300 \mathrm{~A}$ and a pulse FWHM of 7-15 ps. Following the commissioning of APEX in FY 1991, the FY 1992 effort focused on physics experiments associated with the FEL operation. Highlights included lasing at a wavelength of $837 \mathrm{~nm}$, the shortest to date for a Los Alamos FEL. Ultrahigh current densities of $7000 \mathrm{~A} / \mathrm{cm}^{2}$ were demonstrated from the APEX photocathode, which significantly exceeded the previously reported values for multialkali photocathodes. The electron beam's brightness at the end of the APEX 40$\mathrm{MeV}$ accelerator was measured to be 3 $\times 10^{12} \mathrm{~A} /(\mathrm{m}-\mathrm{rad})^{2}$ at a current of $135 \mathrm{~A}$, making the APEX beam one of the world's brightest high-current electron beams. Single-bunch transverse wakefield effects were measured directly for the first time using a fast streak-camera technique, and a scheme for mitigating wakefield effects in a high-current accelerator was developed. A Detailed report of APEX activities can be found in the APLE Free-Electron Laser Program section of this report.

\section{AFEL Experiment}

The Advanced Free-Electron-Laser (AFEL) experiment is a research and development initiative to advance the FEL technology required to build a compact, robust, and user-friendly system for industry. AFEL develops technology in key areas such as the high-brightness beam, high-efficiency microwiggler, high-power optical system, and user-facility.

The AFEL system, now fully assembled, consists of a high-brightness electron linac with a photoelectron source, an emittance-reserving beamline with permanent-magnet transport elements, and a high-efficiency

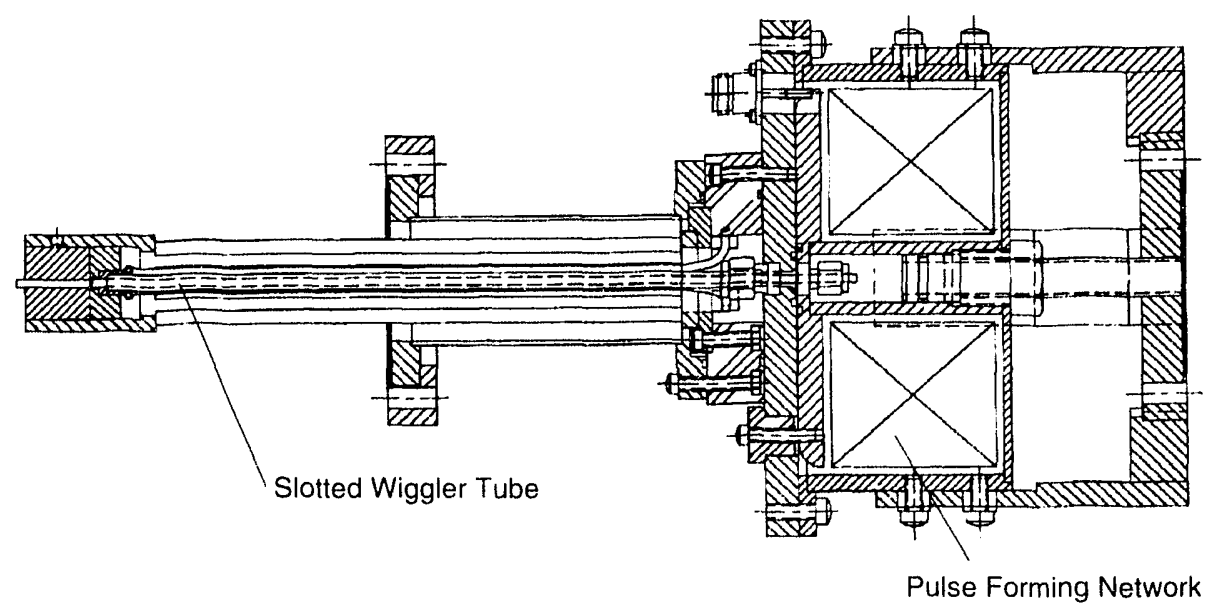

Fig. 6.1. An engineering design of the pulsed-current microwiggler.
FEL resonator with a permanent-magnet wiggler. The linac was conditioned with high-temperature bake and glow discharge to achieve an operating vacuum of $1 \times 10^{.9}$ torr. It was high-power tested to $10 \mathrm{MW}$. Since early summer, the linac has reliably generated high-brightness electron beams. Using a $1-\mathrm{kW}$ drive laser, the highest peak current we obtained was $200 \mathrm{~A}$, and the highest current density was $1 \mathrm{kA} / \mathrm{cm}^{2}$. At this current level, the beam energy was around $16 \mathrm{MeV}$. The macropulse transverse emittance (normalized and $\mathrm{rms}$ ) and energy spread were measured at 5 $\mathrm{mm}$ mrad and $1 \%$. After all the beam transport elements were installed and tested, we were able to transport $70 \%$ of this beam through a tube $25-\mathrm{cm}$ long and magnet wiggler was assembled, tested, and field corrected. The peak magnetic field was $5 \mathrm{kG}$, corresponding to an $\mathrm{a}_{w}$ of 0.45 required for near-infrared operation. Lasing with the AFEL system is expected in early 1993. A detailed report of the AFEL activities can be found in the Advanced Free-Electron Laser Initiative section of this report.

\section{Microwiggler}

The microwiggler program's purpose was to investigate the pulsed-current microwiggler's potential to greatly reduce the electron-beam energy required to reach short wavelength. We completed the physics design and are now developing an integrated engineering design (Fig. 6.1). The engineering $2-\mathrm{mm}$ in diameter. The permanent- 
design shows a uniform wiggler field created by a pulsed current of $35 \mathrm{kA}$ on a tube $305-\mathrm{m}$ m long with an inner diameter of $2 \mathrm{~mm}$. The uniformity required for the wiggler field leads to submicron alignment tolerance required by the slotted tube. In this engineering design. special attention has been focused on eliminating field errors and selecting construction materials. The field errors include static dipole and quadrupole errors in the body and at the ends of the wiggler. We measured and corrected these errors using the pulsedwire technique (Fig. 6.2). In addition, we developed a field-correction system to produce time-dependent dipole and quadrupole fields in the wiggler. These fields will compensate for changes in the error fields caused by thermal heating over the macropulse duration. The materials were chosen to provide good structural stability and good vacuum. This engineering design is now being tested in the proof-of-principle (POP) experiment.

\section{POP Experiment}

By the late 1990s, the United States" microelectronics industry will be required to have an advanced set of lithography tools that will enhance its competitiveness and support a position of leadership and sufficiency in defense and consumer electronics. Los Alamos is proposing to extend the traditional optical lithography process with FELs as powerful and tunable light sources of extreme ultraviolet (XUV) radiation to produce gigabit integrated circuits with features of $0.15 \mu \mathrm{m}$ and less.

\section{A POP experiment is being carried out} at the APEX facility. The goal of the experiment is to lase with a microwiggler at a fundamental wavelength of $850 \mathrm{~nm}$ and then at the third harmonics of 25() $\mathrm{nm}$. The POP experiment is an extremely challenging experiment for many reasons. First, the microwigglers are $305-\mathrm{mm}$ long tubing with a 2- $103-\mathrm{mm}$ inner diameter. For the electron beam to traverse such a tube without loss requires a stable highquality beam. We completed an experiment that showed the bright electron beam at APEX could pass through such a device with better than $90 \%$ transmission. Second, the small-signal gain and detuning range are small because of the short wavelength. The cavity length has to be controlled to 1 $\mu \mathrm{m}$, a sensitivity that is 100 times more stringent than our previous experiments. To solve this problem, we preset the cavity length by lasing at a longer wavelength or by using the drive laser. Mirrors that have high reflectivity at two different wavelengths were specially fabricated for this purpose. Third, the pulsed-current microwiggler is used to enhance FEL gain at short wavelength with available electron-beam energy. The microwiggler is a recently developed device whose characteristics remain to be fully understood.

The good response of the photomultiplier at short wavelengths allows us to measure the spontaneous emission and to use it as a diagnostic tool. We conducted a series of experiments to understand the measured spontaneous emission. However, we have yet to be successful in lasing. 'The problems encountered are caused mainly by the microwiggler's unknown properties, which include field errors and nonuniformity, heating effects, and vacuum property of material used. A third version of the microwiggler is being built and will be tested in January 1993. 


\section{Theory and Simulation}

\section{Theory}

We deviloped software and analytical tools for "sveral related projects: accelerator production of tritium (APT), accelerator transmutation of waste (ATW), and accelerator-based-fusion materials testing in collaboration with the Japan Atomic Energy Research Institute (JAERI). All these projects require a high-current proton or "zutern linac with very low-beam loss. In FY 1991, AT-7 personnel analyzed the beam rms equations for such systems and developed software for performing rms simulations and for finding matched beams, including the effects of acceleration, nonideal quadrupole focusing, realistic gap fields, and rms space charge. This software was used along with TRACE-3D and PARMILA in support of the IAERI collaboration and is expected to be useful in all the projects described above.

We also studied beam halo formation and subsequent beamline interception. Our work on beam-halo growth began with the APT project, which showed how to create exactly stable nonlinear symplectic maps. However, the examples given were purely mathematical and not of practical interest. If realistic maps were found, we could build highintensity ithacs or large, colliding beam-stor ofe rings with the appropriate nonlinear magnets that would ensure nonlinear stability (extremely small partic z loss). Recent work has concentrated on stabilizing real maps that might be of interest for high-intensity linacs. Maps from very realistic models have been stabilized in an ideal sense. One important realization was that stability can be achieved without changing the linear or the first order nonlinear terms. Thus, in a circular machine, for example, one could achieve stability without changing the tunes of the chromaticity correction.

AT-7 has also examined the applicability of the Panoficky-Wenzel theorem of standing-wave deflectors excited in hybrid (TE-TM mixed) modes. In their 1956 paper, Panofsky and Wenzel say they restricted their work to beams moving through cavities excited in TE or TM modes. We reexamined their work and showed the following given their assumptions: (1) the particles are rigid enough that the particlc orbit is not substantially affected in its passage through the cavity, and (2) the transverse electric field vanishes at each end of the cavity, then they have implicitly derived a result that is not restricted to TE and TM modes. More specifically, the transverse momentum imparted to a charged particle moving in the $z$ direction through a resonant cavity of length $d$ is given by

$$
\Delta p_{\perp}=\frac{e}{\omega_{0}} \int_{0}^{d}(-i) \nabla_{\perp} E_{z} d z
$$

where $e$ is the charge of the particle, $\omega_{v}$ is the angular frequency and $\nabla_{\perp} E_{z}$ is" the transverse gradient of the z component of the electric field along the path of the particle. This more general result rave valuable insight into the study o. proposed deflector for APT.

We analyzed the effect of transversely rotated quadrupoles mixing the transverse phase spaces. Assuming that the initial phase spaces are uncorrelated, we have shown that there is an emittance growth in both planes of

$$
\Delta \varepsilon^{2}=\frac{\sin ^{2} 2 \theta}{f^{2}} \sigma_{11} \sigma_{33}
$$

where the quadrupole of focal length $f$ is rotated by an angle $\theta$ transversely. $\sigma_{11}$ and $\sigma_{3,3}$ are beam sigma-matrix elements.

\section{Simulations}

The APEX beamline has been simulated extensively. We have calculated the effects from transverse wakefields (confirmed by actual measurements shown in Fig. 6.3), transversely rotated quadrupoles, and virtual cathode formation just off the photocathode. We have alsi carefully simulated the emittance-me-wurement technigues and have determined that for electron beams from photoinjectors, typical emittance-measurement techniques (e.g., quadrupole scans or multiple screens) consistently underestimate the emittance. The error can be as large as a factor of 2 to 4 resulting from the nonthermal electron distribution. The unfortunate side effect is that it is now more difficult to match electron beams into various beamline elements, such as bends or wigglers that require explicit knowledge of the beam's phase space.

Our collaborations with Boeing have contributed to the APLE beamline design. We developed simulation tools to model the actual nonsymmetric magnetic field in the APLE-injector experiment by including direct BiotSavart integration of the surrent in PARMELA. Using this $t$ ol, we corrected the field errors by s:arefully using transverse stacks of permanent
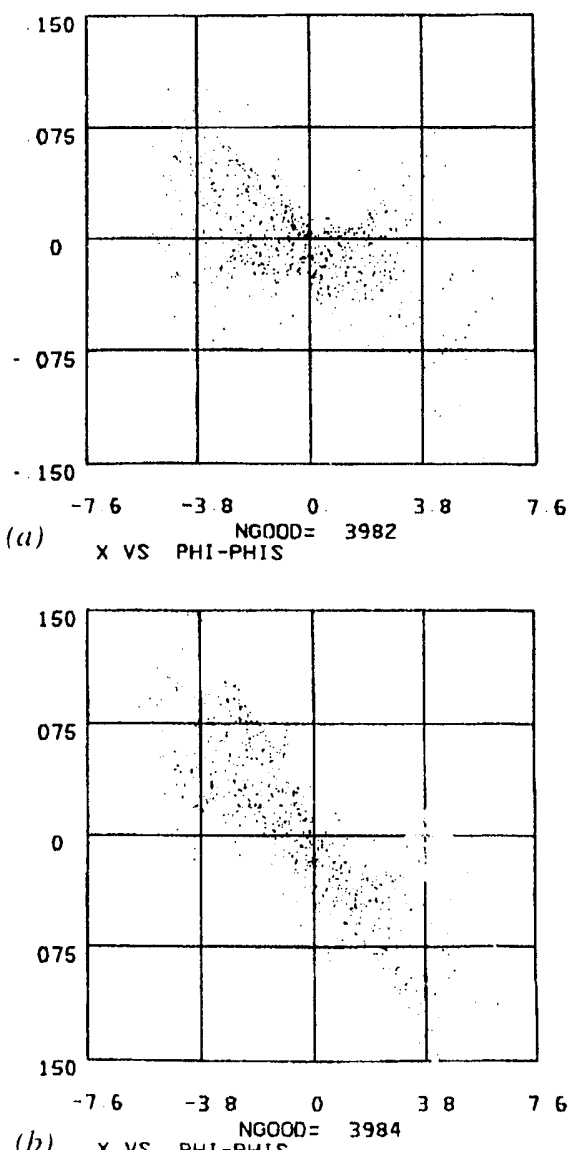

Fig. 6.3. Time-resolved horisontal beam site an screen (a) withoun transverse wakefields, (b) with transierse wakefields. 
magnets. In addition, we used PARMELA to design the beamline and to demonstrate the match into the bends and the wiggler. We also performed beam-interception calculations in the wiggler to ensure that the power interception is acceptable, and threedimensional field simulations on the APLE cavities to determine the field asymmetry from the cavity drive slots and power losses.

AT -7 has designed and simulated a 70-MeV beamline for plane-based FEL applications. We accelerate a 5 $\mathrm{nC}, 20 \mathrm{ps}$ bunch to $40 \mathrm{MeV}$ at which point it is bunched in a chicane buncher to $800 \mathrm{~A}$. The bunch is accelerated another $30 \mathrm{MeV}$ to a total of $70 \mathrm{MeV}$. After matching this beam to a wiggler. FELEX simulations showed up to $20 \%$ energy extraction from this bunch.
We designed a postaccelerated RKA (Fig. 6.4) with the simulation tools developed for the relativistic klystron amplifier (RKA). We have learned that the maximum harmonic current modillation from bunching within a cavity as a function of the space charge has two peaks: one when the beam is ballistic and a second, larger one, when the beam is close to the space charge limit. The fundamental harmonic current can be as much as 1.6 times the average current at the second peak. In this regime, most of the beam's initial energy is captured in the beam's potential fields. We discovered that by including an idler cavity, the higher harmonics (i.e., second and third) have nearly the same current as the fundamental. For short pulse lengths (around $100 \mathrm{~ns}$ ), the electron beam's Coulomb fields can be short circuited in the output cavity. This allows us to bunch close to the space charge limit and obtain the highharmonic current, but yet still have efficient power extraction. For longer pulse lengths (beyond $1 \mathrm{~ms}$ ), the electron beam cannot be shorted out, and the beam energy in the potential fields is lost. However. if the beam is postaccelerated after bunching, the high-harmonic current is preserved and all the additional power added to the bunch can be extracted, resulting in high-overall efficiency. This device has both higher harmonic currents and significantly shorter lengths than a single diode RKA with the same beam current and total voltage. We also noted that this device could be used to extract efficiently at the third, and possibly higher, harmonics and could compete with other sources at 11.4 $\mathrm{GHz}$ in regards to size, power, and efficiency.

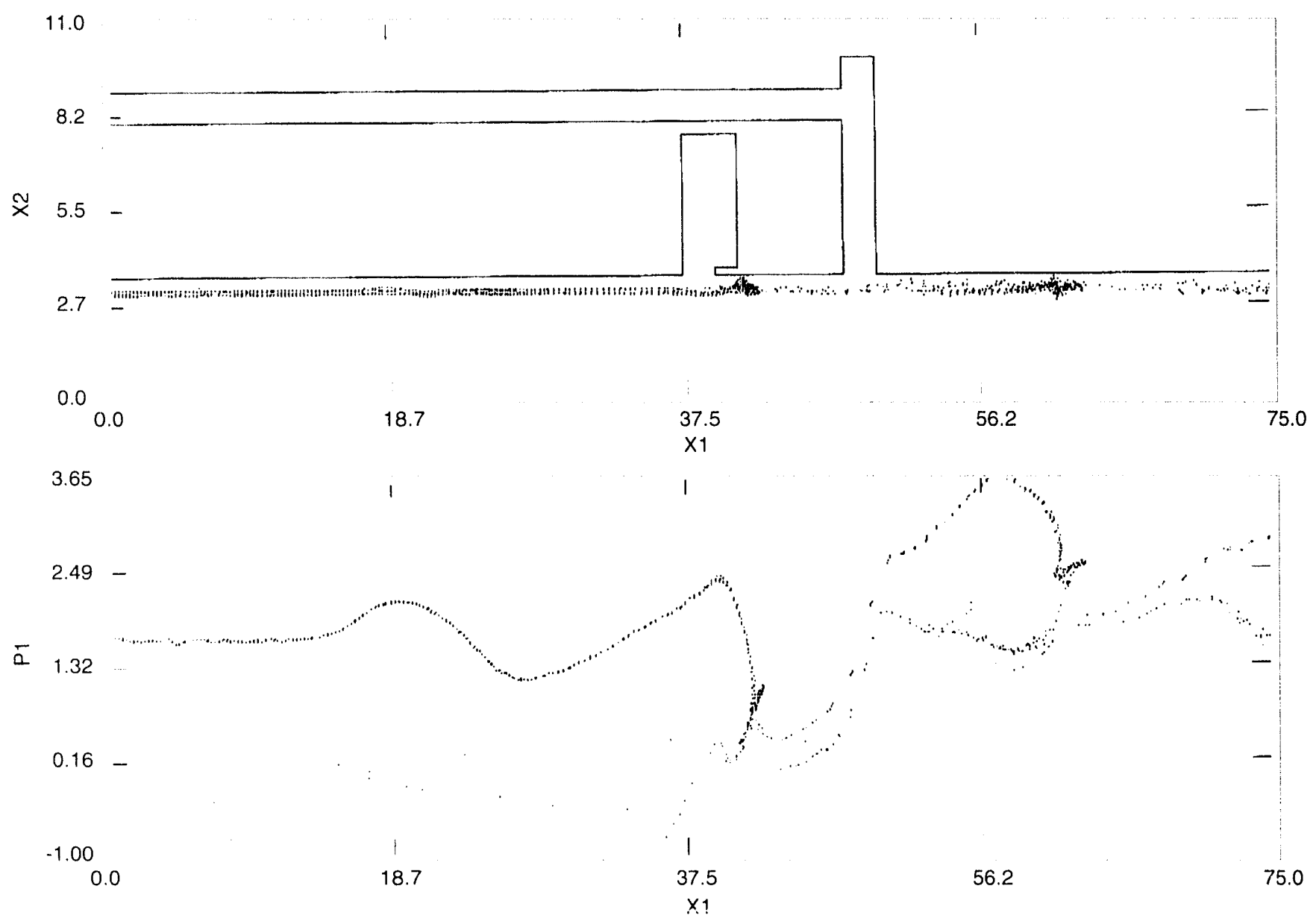

Fig. 6.t. Particle-in-all simulation of a post-accelerated relativistic klystion. 


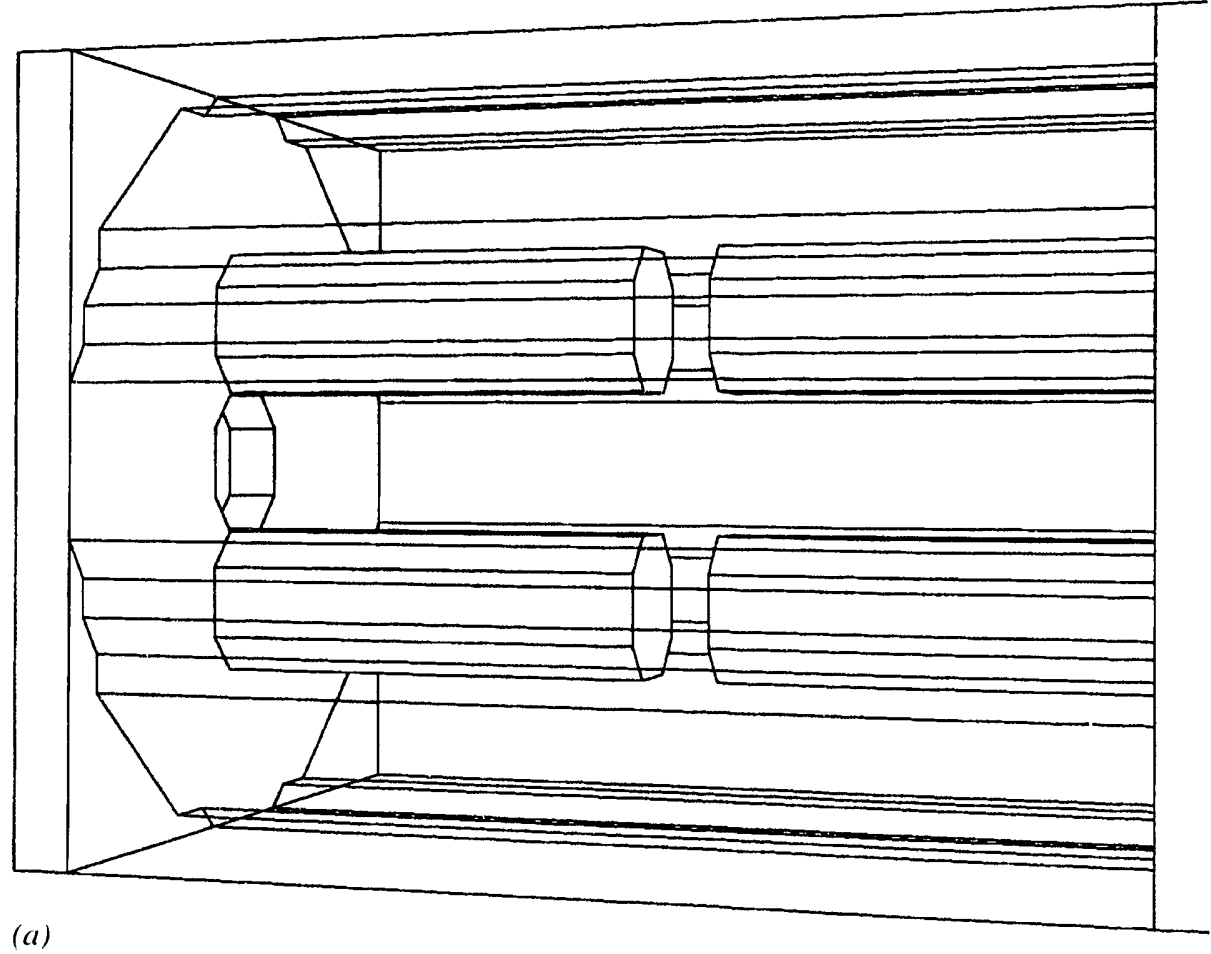

(a)

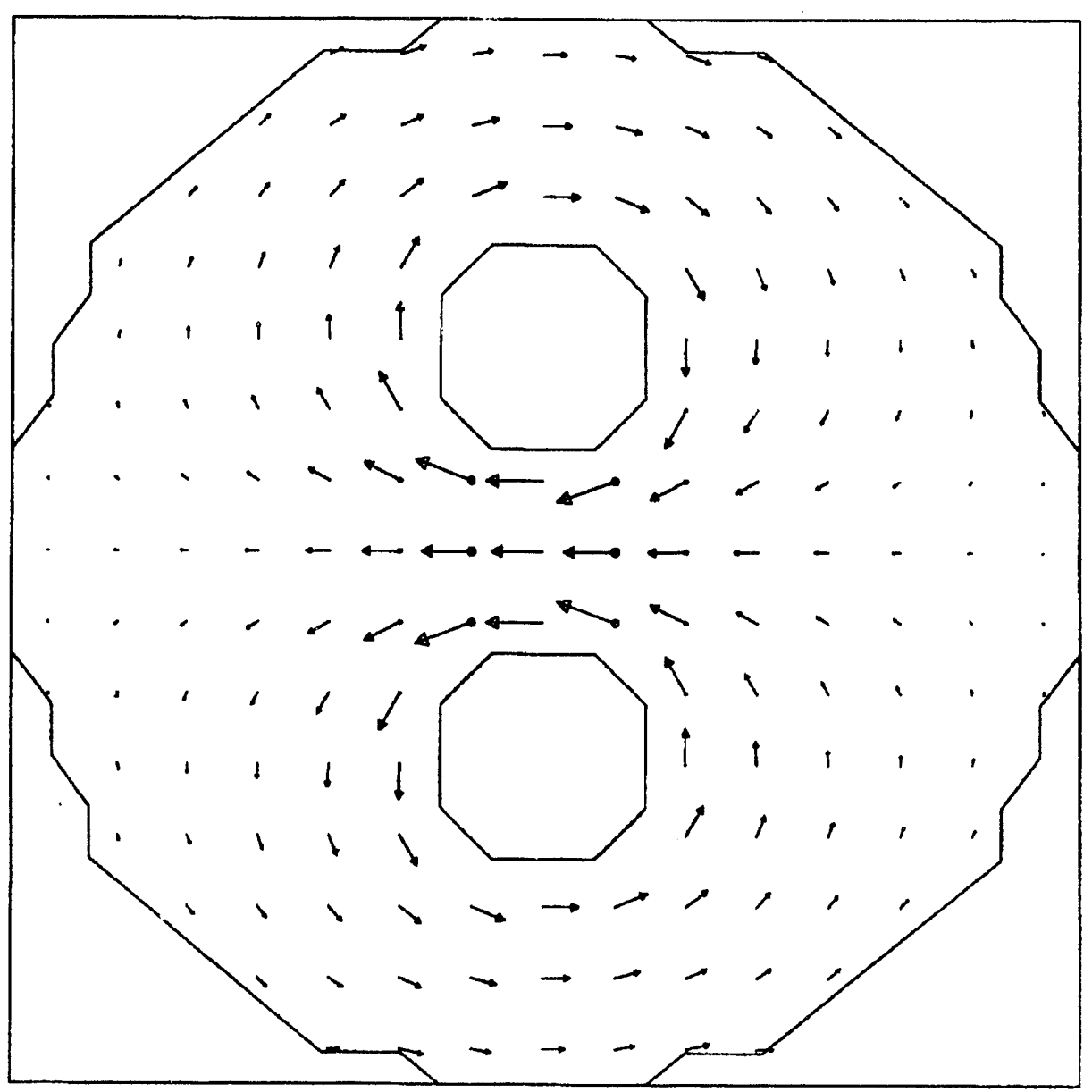

(b)

Fig. o.5. (a) AIW deflector modeled by MAFIA,

(b) Electric field plot produced by MAFIA postprocessor.
MAFIA postprocessors DIFF, FIELDS, and POWER were written as tools for the MAFIA user (Fig. 6.5). Program DIFF subtracts the electric and magnetic fields of one MAFIA run from another, allowing the user to see the effect of a perturbation. Program FIELDS interpolates the MAFIA calculated electric and magnetic fields in between mesh locations and allows for easier interface with particle-pushing codes. Program POWER computes stored energy, total power loss due to finite conductivity, individual $p$, wer loss for each metal, and power-loss densities for the metals specified by the user. These codes were used in the analyses of the APLE design, the APEX linac, and the deflecting cavity studied for APT.

The electron-pushing code PARMELA has been ported to the UNICOS machines, and the various versions of PARMELA have been combined into a unified version. This new code includes both external POISSON and MAFIA fields and three-dimensional injector solenoid fields created by BiotSavart's integration of current loops.

Previously, a code to simulate beanbeam effect in the strong-strong regime was developed using moment methods and was shown to be very fast. The limitations of the code were that it was only a two-dimensional code and that an accelerator ring was modeled only as a linear device. These restrictions have recently been removed. The new beam-beam code for strong-strong regime simulations includes threedimensional effects, such as synchrobetatron resonances due to finite crossing angle and the interaction of the beam-beam nonlinearities with the machine's nonlinearities. In addition, tracer particles have been added for purposes of visualization. 


\section{Los Alamos Accelerator Code Group}

Funded by the Department of Energy to serve the US scientific community, the LAACG provides software and services to design and analyze particle accelerators and beam-transport systems. Its main activities include developing and maintaining software; consulting with others; distributing software and documentation; and gathering and disseminating information. In recent years, the code group's services have extended to international users.

\section{Software Development and Maintenance}

The LAACG completed two major revisions to the widely used POISSON/ SUPERFISH codes used to design accelerator components, including magnets and radio-frequency (rf) cavities. The first major revision was Release 3 , which contained bug fixes, physics enhancements, and expanded graphics support. The second major revision, Release 4 , included removing all bit packing, increasing FORTRAN77 standardization, including the preprocessor FRONT, improving graphics capability based on the X-Windows protocol, and producing arrow plots
(Fig. 6.6). Release 4 is available for various platforms, including Sun SPARC, HP Series 700, IBM RISC/ 6000, SGI, VAX/VMS, and Cray/ UNICOS.

The LAACG expanded its activities related to ARGUS, a three-dimensional family of simulation codes developed at Science Application International Corporation (SAIC). ARGUS contains modules for steady-state and timedependent particle-in-cell simulations and for time domain and frequency domain electromagnetic simulations. Code group personnel have used ARGUS to model the Large Orbit Gyrotron experiment. They also attended a course at SAIC to become expert users and to provide SAIC with guidance in preparing the 1993 release of ARGUS through the LAACG. Figure 6.7 shows an example of ARGUS output.

During FY 1992 Cray computers at Los Alamos and the National Energy Research Supercomputer Center (NERSC) at Livermore switched to the UNICOS operating system. Code group personnel developed UNICOS versions of several programs, including MAFIA 2.04 and PARMILA. In addition, code group personnel began using the
INGRID mesh generator as a first step toward performing simulation with unstructured grids.

\section{User Consultation}

The LAACG consulted with many researchers at national laboratories, universities, and in industry. Most consultation involved helping researchers correctly use codes to perform calculations and simulations.

\section{Distribution of Software and Documentation}

The LAACG distributes software in three ways: electronic transmission, magnetic media (tapes), and access to software at NERSC. The LAACG also distributes documentation. From January to June 1992, the LAACG delivered software to over 150 contacts (including system managers) and mailed out over 900 documents. These figures increased dramatically in the latter half of 1992 because of widespread interest in POISSON/ SUPERFISH Release 4.

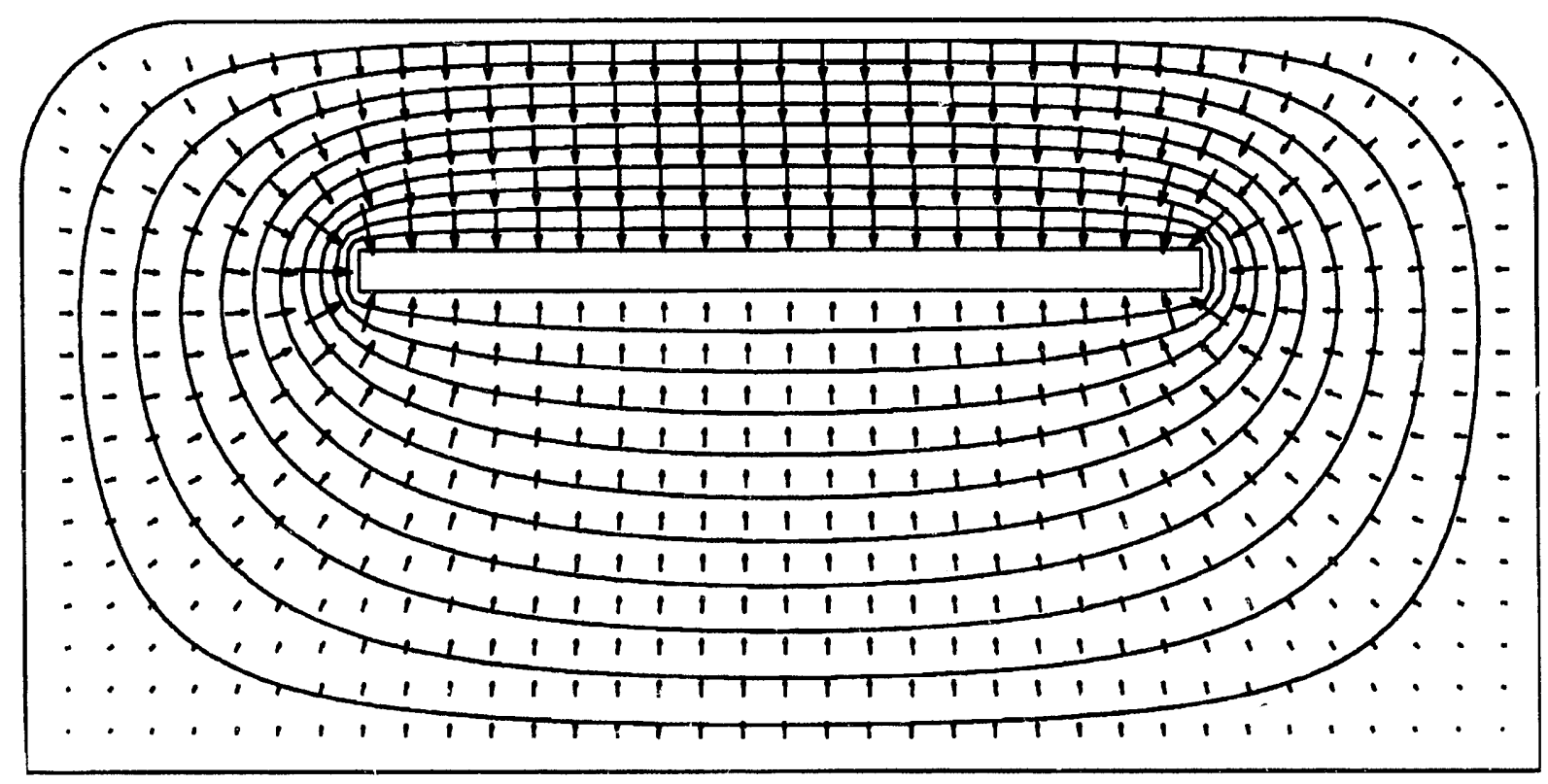

Fig. 6.6. Arrow plot of field calculated with SUPERFISH codes. 


\section{Gathering and Dissemination of Information}

During the 1992 Linear Accelerator Conference at Ottawa, Canada, the code group organized an information booth to introduce code group services to users. In addition, it held a "town meeting" to hear users' concerns regarding accelerator code development and availability. The LAACG is now co-organizing the 1993 Computational Accelerator Physics Conference, which will be held in February 1993.

\section{Other Activities}

\section{Alpha Simulation Using Neutral Beams}

Effects such as instabilities or anomalous transport from the alpha particles produced in burning plasmas may affect the performance of ignited fusion machines such as ITER. It is important to obtain information about these effects soon so the best designs for these future machines can be produced. Experimental simulation has been done in existing devices by injecting neutral $250-\mathrm{KeV}$ beams with scaled magnetic fields. However, we

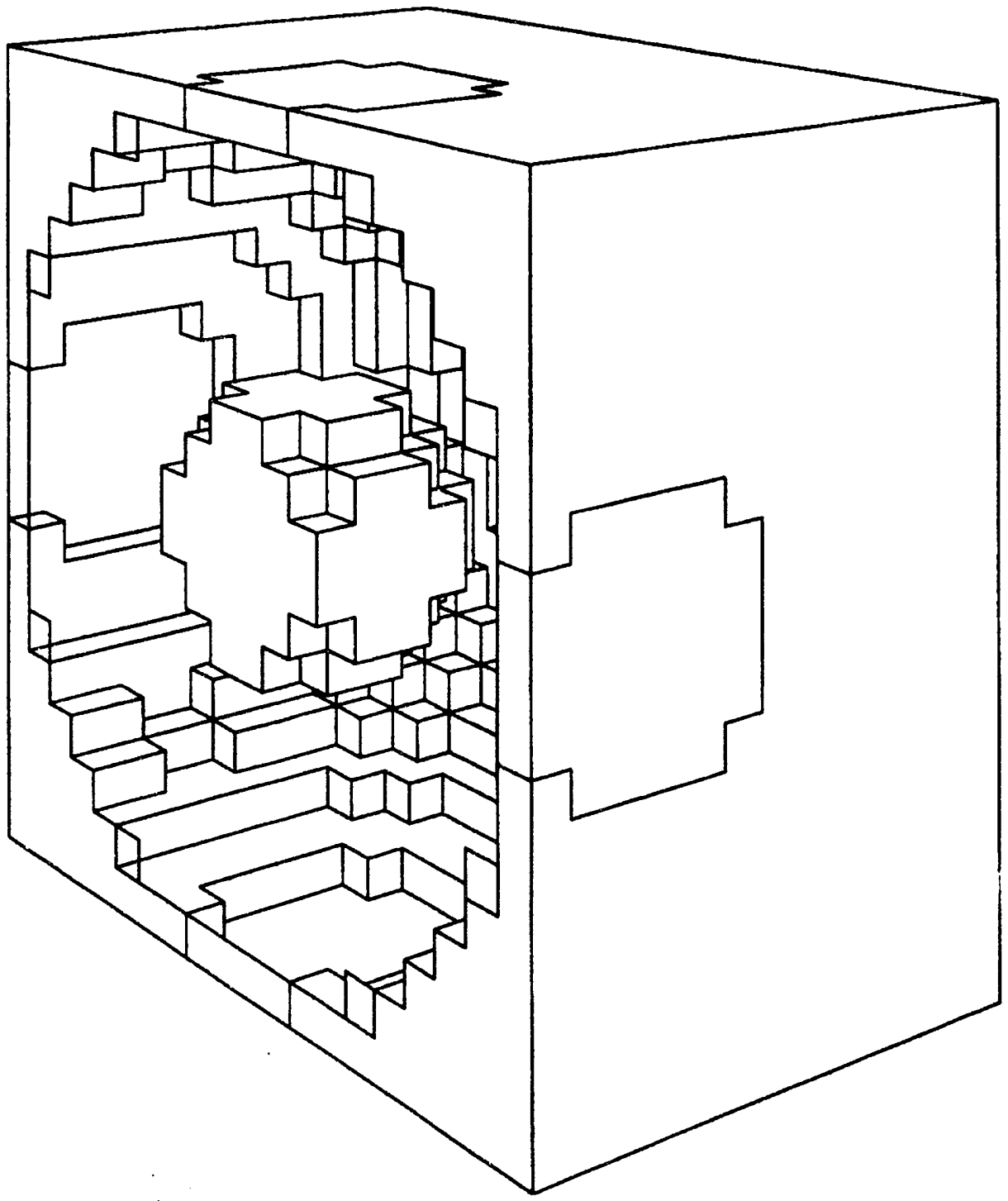

Fig. 6.7. Typical output from the ARGUS code.

realized that neutral beams developed for the neutral particle beam (NPB) program could provide much more realistic experimental simulations of the possible effects at conditions much closer to fusion conditions. Before we discovered the usefulness of the neutral particle beam, a cw 1.7 MeV D- beam with a current of $125 \mathrm{~mA}$ in one module was needed, and another 4 to 16 modules were required to inject approximately the same free-energy density into a device, such as TFTR or DIIID, at full-magnetic field under near-fusion conditions as would be produced in a burning plasma. Single modules with these parameters are being developed for the NPB program.

\section{XUV Plasma Source}

We are seeking funding for a unique electron beam-driven plasma XUV source that is currently in conceptual development. The source consists of a $5-\mathrm{MeV}$ electron beam and a tenuous plasma of neon or nitrogen. The electron beam is a continuous stream of pairs of electron bunches. The first bunch has low charge and is $10 \mathrm{ps}$ long; it partially ionizes the gas into plasma. The second bunch has a high charge of $5 \mathrm{nC}$ and is less than $1 \mathrm{ps}$ long; it will deposit $75 \%$ of its energy into the flasma via wakefield interaction. The resulting hot, dense plasma quickly (i.e., within $100 \mathrm{ps)} \mathrm{loses} \mathrm{energy} \mathrm{by}$ XUV line radiation. The output wavelength depends upon the chosen gas type and its pressure. The output power level depends upon the gas type and the parameters of the input electron beam. Compared with similar schemes for example, the laser-driven plasma, this scheme has several advantages. First, an rf electron beam avoids the particulate contaminates of a laser produced plasma. Second, plasma recombination to its initial atomic state allows the gas to be recycled through a closed-loop recovery system. Third, it is "granular" (i.e., acceptably small unit size and cost) enough to be of interest to soft $x$-ray lithography systems. Fourth, this approach is adjustable to yield short $(15 \mathrm{~nm})$ or long $(60 \mathrm{~nm})$ 
wavelength irradiance by changing only the composition of the flowing gas (e.g.. neon versus nitrogen). This operation's versatility is an attribute not offered before to the semiconductor manufacturing industry. Fifth, this design has multisided output access, each yielding irradiance suitable to illuminate a single stepper. Sixth, this source has upgrade power potential because the injectoraccelerator structure does not have an operational thermal limit.

\section{SSC Collaboration}

Our collaboration with the Supcrconducting Super Collider (SSC) theory group concentrates on the rf counter phasing and beam-loading stability problems for the low energy booster (LEB). To avoid multipacting in the if cavities, the cavity voltage must be above $20 \mathrm{kV}$. However, to achieve the desired capture during injection, the rf voltage must be about $10 \mathrm{kV}$. To avoid this problem, we plan to counter-phase the rf cavities, $50 \%$. Consequently, the cavities can be run above the multipacting limit, yet the injection capture can still be optimum. In addition, the cavities can be easily rephased when higher voltage is needed. We have shown that if the rf amplifier can deliver $120 \mathrm{~kW}$ per cavity, there will be enough rf power to maintain the correct voltage when the tuning error in the cavities is moderate. Higher harnonic rf systems are used in synchrotrons and storage rings to increase the bunch length and the spread of the synchrotron frequency to reduce the space charge effect and to dampen the longitudinal instabilities. We also examined the beam-loading stability in an if system with a higher harmonic by directly investigating the equations derived from the equivalent-circuit model. The stability conditions for the LEB have been derived from the linearized equations.

\section{PSR Development}

We observed a fast transverse instability with beam loss in the 800-MeV Los Alamos proton storage ring when the injected beam intensity reached $3 \times 10^{13}$ protons per pulse. Understanding this instability and the methods to control it have taken on new importance as the neutron scattering community considers the next generation of acceleratordriven spallation neutron sources, which call for peak proton intensities of $2 \times 10^{14}$ per pulse or higher. Previous observations indicate that the instability is probably driven by electrons trapped within the proton beam. Theoretical study has shown that beam leakage in the interbunch gap leads to electron trapping. In 1992, several experiments were carried out by using the newly implemented "pinger" and by varying the machine transition gamma to explore further the nature of the "e-p" instability. Currently, we are examining the experimental data and writing a simulation program to study the longitudinal space-charge effects.

\section{LANSCE II Propo: :I}

Recently, serious consideration has been given to a next-generation accelerator-driven spallation neutron source at Los Alamos. Conceptual design is in process. We helped design the lattices for the $800-\mathrm{MeV}$ ring and the $2-\mathrm{GeV}$ ring, provided the preliminary stability' studies, and studied the design's longitudinal phase-space painting scheme.

\section{ESNIT Collaboration}

The Energy Selective Neutron Irradiation Testing project is a joint venture between LANL and JAERI. An accelerated deuterium beam is directed to a lithium target, producing neutrons used for fusion materials research. Currently, we are considering a 2- to 3-MeV RFQ at either $120 \mathrm{MHz}$ or 175 $\mathrm{MHz}$, with a 75- to $125-\mathrm{mA}$ deuterium beam, followed by a 35 - to 40 MeV DTL. 


\section{Accelerator Theory Notes}

The following AT-7 Accelerator Theory Notes were distributed during the report period:

1. M. J. Browman, "Testing Program Power," AT-7:92-ATN-1.

2. M. J. Browman, "Effect of Tuning Stubs on the Power Loss in a Four Slot Cavity," AT-7:92-ATN-2.

3. M. J. Browman, "Generating MAFIA Azimuthally Symmetric Cavities from a Two-dimensional Cross Section," AT-7:92-ATN-3.

4. R. Ryne, "Normal Analysis of Anharmonic Oscillator, including Envelope and Emittance Growth Calculations," AT-7:92-ATN-4

5. R. Gluckstern/R. Copper, "Beam Breakup Estimates for a Super Conducting Proton LINAC," AT-7:92-ATN-5.

6. R. Gluckstern, "Transient Effects in the Slotted Cylinder," AT-7:92-ATN-6.

7. C. Fortgang, "Field Correction for a One-metered Long Permanent-Magnet Wiggler," AT-7:92-ATN-7.

8. J. Merson, "Power Densities for Superconducting RFQ LY-10-14," AT-7:92ATN-8.

9. J. Merson, "HALAST and GMHALD on UNICOS," AT-7:92-ATN-9.

10. J. Merson, "SPCG 4 and GRAF on UNICOS," AT-7:92-ATN-10.

11. T. Wang, "Robinson Instability with a Higher RF Harmonic," AT-7:92-ATN-11.

12. M. J. Browman, "The Panofsky - Wenzel Theorem Revisited," AT-7:92-ATN-12.

\section{Technical Memoranda}

1. J. Merson, "Cell Dimensions used to Generate SFDATA Table for ATW 350 DTL," AT-7:92-TM-1.

2. M. J. Browman, "Program Plots," AT-7:92-TM-2.

3. M. J. Browman, "Program Power," AT-7:92-TM-3.

4. J. Merson, "Dimensions of Superconducting RFQ LY-7-7," AT-7:92-TM-4.

5. J. Merson, "Prior work done on Behavior of Multiple Beams in an RFQ," AT-7:92-TM-6.

6. J. Merson, Wangler, "Superconducting RFQ LY-7-15 Dimensions," AT-7:92-TM-7.

7. J. Merson, "Power Densities and Fields for Superconducting RFQ LY-7-15," ÁT-7:92-TM-8. 
8. J. Merson, "Total Power Loss for Superconducting RFQ LY-7-15," AT-7:92-TM-9.

9. J. Merson, Wangler, "Dimensions of Superconducting RFQ LY-8-7," AT-7:92-TM-10.

10. J. Merson, "Power Densities for Superconducting RFQ LY-8-7," AT-7:92-TM-11.

11. J. Merson, "Frequency, a Stored Energy and Vane Voltage of LY-8-7 from MAFIA Superconducting RFQ," AT-7:92-TM-12.

12. J. Merson, "Fields at End Wall of LY-8-7," AT-7:92-TM-13.

13. J. Merson, "Superfish Definition and Results for Superconducting RFQ SCRFQ 11," AT-7:92-TM-15.

14. H. Takeda, "Otimization of APLE Photoinjector with the Injector Solenoid Field Modeled by the Biot-Savart Law," AT-7:92-TM-16.

15. H. Takeda, "Optimization of ESNIT RFQ at 175 MHZ," AT-7:92-TM-17.

16. J. Merson, "End Region Dimensions," AT-7:92-TM-18.

17. J. Merson, "Field's for Superconducting RFQ Geometry ZY5-AJ," AT-7:91-TM-24.

18. J. Merson, "Summary of Super Conducting RFQ Mafia Calculations," AT-7:91-TM-25.

19. M. J. Browman, "Comparison Between Previously used Side-complied Geometry and APEX Cavity," AT-7:91-TM-26.

20. M. J. Browman/Rodenz, "Proposed Changes to M3," AT-7:91-TM-27

21. M. J. Browman, "PARMELA Input for APLE Cavities with Different Plot Configurations," AT-7:91-TM-28.

22. M. J. Browman, "Effect of Tuners on the Fields in an APLE Cavity," AT-7:91-TM-29.

23. J. Merson, "NERSE Unicos Class Highlights," AT-7:91-TM-30.

24. A. Lombardi, "Wakefields Effect in Microwiggler," AT-7:91-TM-31.

25. J. Merson, "Source of SF Data used for Current ATW PARMILA Calculations,"AT-7:91-TM-32.

26. J. Merson, "Preliminary ATW DTL Dimensiorı, ' AT-7-91-TM-33.

27. J. Merson, "POISSON/SUPERFISH Mafia and NERSC," AT-7:91-TM-34.

28. J. Merson, "Power Density in Selected Cells of ATW DTL 35od," AT-7:91-TM-35.

29. J. Merson, "Power Requirements Dimensions of ATW 350 DTL," AT-7:91-TM-36. 
Technical Highlights $\bullet$ AT-7 • Accelerator Theory and Free-Electron Laser Technology 

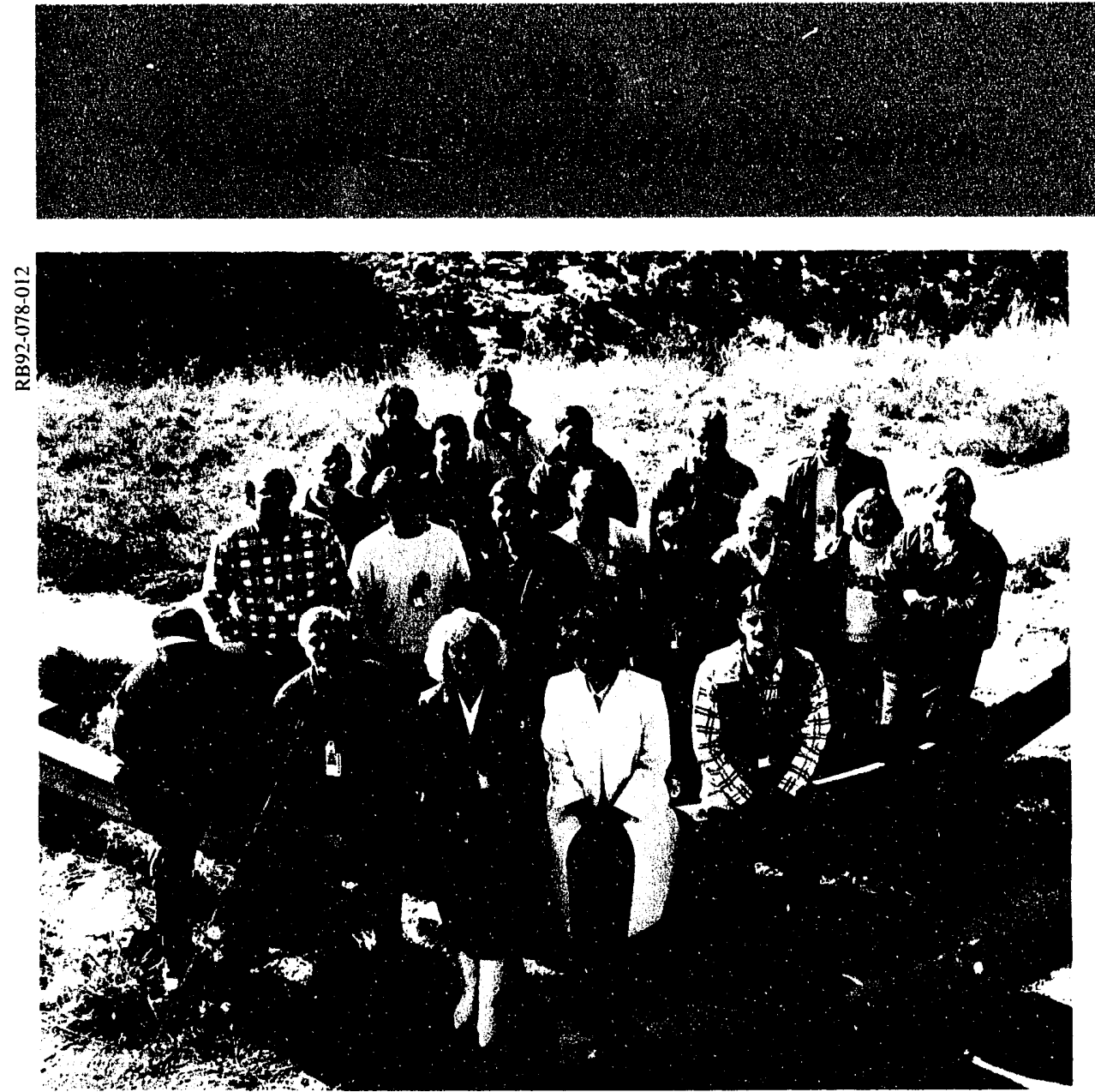

Introduction

Background

Accelerator Control

Operations.

Waveform Acquisition

.88

Off-line Testing 88

Commissioning and Automation

Software Tools ..........................8 88

Phase Scan .............................. 88

Steering .................................. 88

Automatic rf Structure

Conditioning ......................... 88

Video Beam Profiles ................ 88

Ion Source Automation ............89

Control System Hardware ............89

Operational Support ................ 89

Fast-Protect System

Development ........................ 90

Optically Isolated Binary

Output Module ........................ 90

Step-Motor Control .................. 90

Transient Digitizer ................... 90

Next Generation Computer for IOCs 90

Binary Output........................ 91

Nonvolatile IOC Memory

Module.....

Electronic Computer Aided Design

(ECAD) Support 91

Computing Support 91 


\section{Introduction}

In FY 1992, AT-8's major efforts included producing, installing, and commissioning of the control system for the intertank matching section (IMS) and the first drift tube linac (DTL) section of the Ground Test Accelerator (GTA). Typically, AT-8's tasks are to generate the hardware and software necessary to control and monitor accelerator components and subsystems, then to integrate, and test the controls on the beamline.

\section{Background}

Two experiments which studied the performance of the IMS and DTL were executed. During these experiments, controls personnel manned two shifts a day to assist with accelerator operation and data acquisition. The distributed architecture control system for the experiments consisted of about 15 frontend VME-based INPUT/OUTPUT controllers (IOCs) with associated instrumentation, and approximately 10 color work stations used as operator interfaces running the EPICS software.

This year marked the first use of the GTA control room. Previous experiments had been operated from a temporary control room amidst the electronic equipment racks. Operating from the control room was a definitive test of control system reliability and functionality, as neither the accelerator nor electronic equipment could be observed directly.

\section{Accelerator Control}

We installed the first DTL in a new vacuum vessel that is planned to contain five DTL modules. The control system monitors and provides supervisory control for the vacuum, cryogenic temperature, radio-frequency (rf) power, DTL tank positioners, frequency tuners, and a steering magnet. An additional three IOCs were installed to accommodate new DTL controls, rf controls, and beam diagnostics instrumentation.
We installed a complete vacuum control system for the new DTL vessel. Pumps, gauges, and valves are all operable through the control system as well as from a manual control panel. The control system provides interlocks that can automatically shut off devices or close valves in the event of an equipment failure. The control system also provides automatic trending of vacuum readings. A single IOC provides control and monitoring of the radio-frequency quadrupole (RFQ) vacuum system and the first DTL vacuum system.

The DTL, like the RFQ, operates at cryogenic temperatures. A large number of silicon diode temperature transducers are used to monitor the temperature of the DTL structure and various points along the cryogenic manifolds. Temperature readings are automatically logged by the control system. The desired operating set-point is typically 25 to $35 \mathrm{~K}$. If the control system detects any temperatures above $50 \mathrm{~K}$, it automatically disables beam production.

The DTL module is supported by five mechanical positioners, much like the RFQ. This enables the structure to be translated along orthogonal axes after the accelerator has cooled to cryogenic temperatures. These positioners are operable from the control room, which transforms the operator's desired $x / y$ coordinates into position commands for each of the five positioners.

We installed another if power system controller for driving the DTL. The control system interface is nearly identical to the ones installed previously for the RFQ and IMS. Through the control system, the operator may set and monitor parameters such as phase and amplitude for any cavity. RF engineers may control more detailed settings such as system calibrations and closed-loop control coefficients.

\section{Operations}

Integration and operation have become important issues as hardware is added to GTA and experiments have become more complicated. New application software and some hardware have been customized to assist in operating the accelerator effectively and safely.

The accelerator safety systems now include a control system integrity check. As part of the integrity check, the run-permit system monitors a 'heartbeat' from each IOC that controls critical accelerator operations. If any of these front-end processors fails to respond or is operating abnormally the accelerator is automatically shut down. The control system can also disable the accelerator when other abnormal conditions are detected, such as a vacuum reading that is too high or steering magnets that are not set within tclerance.

We endeavored to create software tools that aid experiments in effective and efficient accelerator control. The data archiving software has been tailored to users' requests. A new capability has been developed that can automatically vary an accelerator parameter through a series of set-points and collect data at each set-point, resulting in increased efficiency over repeated manual adjustments and data acquisition. A data retrieval tool extracts previously archived data and provides plotting, translation to several foreign computer file formats, and statistical analysis. Other tools include .... on-line operator log, an alarm manager that provides orderly notification of anomalies, and a graphic timing control program that displiys accelerator timing settings as a timing logic diagram. 


\section{Waveform Acquisition}

We now use a digitizer that has the capability to acquire synchronous waveforms. This digitizer was installed and a software driver was written to capture waveforms of various accelerator signals at a sample rate of $5 \mathrm{MHz}$ with 12-bit resolution. The entire macropulse can be captured, and up to approximately 80 contiguous macropulses can be buffered in the IOC. Four channels are captured simultaneously and time-stamped so they can be later correlated with other data. The operator can set the number of samples per waveform, the number of waveforms to acquire, and the trigger timing. The operator can also preview the four waveforms on the work station before data is acquired. We used this system during experiment $2 \mathrm{~A}$ for collecting data for beam jitter studies and for studying the performance of $\mathrm{rf}$ adaptive feed-forward electronics.

\section{Off-line Testing}

We maintained two test stands for testing accelerator structures before they were installed on the GTA. A lowpower cryogenic test bed and a similar facility for high-power testing have been instrumented with controls equipment to duplicate beam-line conditions. Besides providing data on the behavior and operation of the devices under test, we developed and tested control algorithms before installing them in the GTA control system.

\section{Commissioning and Automation Software Tools}

Several tools that have specific roles in the commissioning of the accelerator were either implemented this year or were considerably changed from last year. These include programs for phase scan, for steering in the low-energy beam transport (LEBT) and IMS, and ior automating rf cavity conditioning. A program was also developed that recorded beam position data and transferred it across the network to a
VAX system for use by RESOLVE, a beam-tracing program. In addition, the program ESCAN was ported from the GTA control system to the discharge test stand (DTS) control system. This brought all the emittance scanning programs to one method allowing for more meaningful comparison.

\section{Phase Scan}

The phase scan program that we began testing last year was brought to the point of full functionality and maturity and is robust enough to operate effectively. It does automatic scanning, data scaling, data display, and data storage under operator control. We modified the original design because the rf power occasionally shut down at zero phase, and a specified time period was required for the motorized phase shifter to work after a command to shift phase had been sent. Now the data that an operator sets to configure the scan is saved, on a cavity by cavity basis, for a default setup the next time the program is run. This program has produced essential data for documenting operation of the cavities as well as for initial tuning of the cavities.

\section{Steering}

AT- 8 built a beam-steering software tool that will aid us in our studies of beam launching into the first DTL tank. This tool provides the beam with a target at the DTL entrance; the target consists of position and angle in $x$ and $y$. This beam-steering tool has yet to be used on-line because of inconsistencies with calibration data from the IMS microstrip probes that provide data to the steering tool. The steering tool for LEBT steering, previously reported, uses a similar philosophy of steering to a target. We dramatically modified that code because the LEBT had been shortened. Lambertson magnets now located inside the solenoids are employed to do the steering. However, inconsistent experimental data obtained from emittance scanners in the LEBT have prohibited this program from being fi:lly tested. We expect the problems to be understood and corrected and the program to be operational in a very short time.

\section{Automatic rf Structure Conditioning}

We implemented a software tool to provide automatic $r f$ conditioning of the RFQ, our first attempt at automated conditioning for any GTA cavity. The tool was partially tested during the last beam experiment, but a complete test requires full access to the RFQ. We hope this effort will yield information useful in designing a general code to condition all GTA cavities.

\section{Video Beam Profiles}

The video profile diagnostic system implemented for GTA in FY 1992 was brought to a fully operational state after several problems were overcome. We discovered there was not enough residual gas to produce light, thus gas needed to be injected into the beam path. The first attempt at this uncovered faults in the electronics package that controlled the injection valve, in the software that controlled that valve, and in the hardware that delivered the gas. Modifications were made and the system became operational. Initially, only the system designer could operate the diagnostic. However, we modified the software interface to become more user friendly, and now this diagnostic is a production tool that can be used by any GTA operator.

Another problem encountered was the early images captured by the video profile showed background that extended beyond the edges of the actual viewing area. We used several methods to eliminate this background. We reduced the intensifier gain and integrated over only the time when beam is present to reduce the background to an acceptable level. A cross section of the remaining image was modeled by a linear combination of two Gaussian functions. The narrower function was the image from the beam interaction, therefore, we calculated centroids and 
widths from that information while the wider function compensated for the background. This calculation was incorporated into the real-time imaging software and displayed as the images were obtained.

Presentations were made on this diagnostic at both the Neutral Particle Beam (NPB) Symposium at Argonne and the LINAC Conference at Ottawa. These presentations covered both the implementation system and data analysis methods.

\section{Ion Source Automation}

In FY 1992, AT-8 demonstrated a neural-network based automated ionsource tuner could control the smallangle source on the Discharge Test Stand. This work has since been transferred to the GTA injector control system. Although the problem of automating this source is quite similar to the work done on the DTS, there are major differences. First, the dimensionality of the problem is increased because there are more adjustable parameters that must be addressed on the $4 \mathrm{X}$ source used on GTA. Second, the increased mass of the GTA source requires more heat for proper arc operation than did the SAS source. In fact, the heaters provided on the $4 X$ source are insufficient to bring the anode and cathode to proper operating temperature, so the extra heat required must be provided by the arc itself. Therefore, the control of the temperatures of the anode and cathode must follow a different strategy. Third, to move the ion-source controller from a demonstration mode to an operational mode requires manual operation. When the controller is operational it must operate unattended and therefore must be robust. However, we have resolved all problems identified while using the controller in the DTS demonstration. For example, if a thermocouple indicates an impossible temperature, the controller reacts by taking the loop off-line but maintains the heater set-point. This open-loop mode of operation can continue for a short time allowing the operator to address the problem. The system is robust to problems with the thermocouples, the errors in the Allen-Bradley computer interface, and in an automatic to manual mode change. The ion source is maintained at operational because of the system's sensitivity to these conditions and the controller's reaction to the problems.

During FY 1992 we presented our work on automation to an industrial workshop organized by SDIO, at the NPB Symposium at Argonne, at the LINAC Conference in Ottawa, and we published our work in Nuclear Instruments and Methods.

\section{Control System Hardware}

\section{Operational Support}

Final checkout and integration of all beam experiment $1 \mathrm{C}$ controls were in progress at the beginning of FY 1992. During the early stages of the experiment, it became clear that additional controls and monitors were necessary for several components of the IMS. We designed additional hardware for the rfdrive-loop elbows to maintain the temperatures and to prevent if field breakdown, for the frequency tuner stepmotors to prevent binding at cryogenic temperature, and for the upstream steering quadrupole motors to facilitate operation within acceptable limits. The original drive motor on the D-plate bending magnet was found to be marginal in torque; therefore, controls for a larger motor were added. The control room timing system, which provided key timing signals for control room data acquisition, and the IMS video profile system were integrated and became solid operational tools.

To prepare for experiment $2 \mathrm{~A}$ and future experiments, we implemented two test beds so that each of the ten DTL modules could be characterized, rfconditioned, and functionally tested before installation on the beamline. Each DTL rilodule contained 34 temperature monitors, $2 \mathrm{rf}$ frequency tuners, and 2 step motors for two- dimensional steering-quadrupole positioning. DTL module I also implements five step motors for threedimensional positioning of the entire module. We decided to upgrade the control system for the High-Power Cryogenic Test Bed (HPCTB), to a system that could be capable of highpower if conditioning of DTL modules. This modification, in conjunction with required safety enhancements, necessitated a complete revamping of control wiring and electronics. Another IOC and associated controls were fabricated and integrated for use with the LowPower Cryogenic Test Bed (LPCTB).

Before experiment $2 \mathrm{~A}$, the beamline controls for DTL module 1 were fabricated. Wiring to the beamline was installed and tested, and a new DTL IOC was implemented on the classified control network. After DTL positioning tests, we determined that larger step motors with linear variable differential transducer (LVDT) positional feedback would be required for adequate DTL position control. Consequently, we installed and tested additional hardware.

For experiment $2 \mathrm{~A}$, both DTL module 1 and the D-1 diagnostic plate were installed in the first of two DTL vacuum vessels. A rack of equipment with its associated cabling was installed to control the various valves, pumps, and monitors for this new vacuum system. The new controls were interfaced to the existing vacuum control IOC.

Because high-beam energies and currents were associated with experiment 2D, we used sophisticated beamloss monitoring electronics to determine when beam was spilled. A new IOC was assembled to interface with prototype beam-loss electronics so that it could be functionally tested during experiment $2 \mathrm{~A}$. The new IOC contains approximately 100 channels of binary and analog inputs and outputs as well as time-stamp generation, triggering, $: \mathrm{id}$ fast-protect signals. A large database, several sequence programs, and control screens were also implemented to support this effort. 
Several modifications to the versamodule European (VME) time-stamp generation modules were made after experiment $\mathrm{IC}$ for compliance with new low-level if timing requirements for experiments $2 \mathrm{~A}$. The new timestamping hardware was fabricated and installed in all eight GTA IOCs that incorporated hardware-generated time stamps and performed as required during the experiment.

\section{Fast-Protect System Development}

To protect accelerator components at higher beam energies, we implemented a fast-reacting hardware fault detection system for experiment $2 \mathrm{~A}$ that can truncate a beam pulse within microseconds of a fault. The hardware designed for this system included several new modules. The Fast-Protect Master module (Fig. 7.1) generates a carrier signal that is daisy chained to a series of slave modules. Any slave module receiving a fault input will not pass the carrier signal, thereby breaking the chain. At the end of the chain is the Extractor Gate Interface Chassis. The chassis has inputs from the Personnel Safety System, the Run-Permit System, and the Shift Supervisor Key as well as from Fast-Protect. Inputs from any of these systems can terminate the GTA beam. Under program control, the Fast-Protect Master module can be used to perform a series of functional tests of the entire Fast-Protect System. Each slave module can be monitored by the control system to determine the status of its inputs. Versions of the slave module were designed in a VME package for beam-loss applications and in a VME External Interface (VXI) package for $\mathrm{rf}$ applications. Fast-Protect Warning Modules were also designed and built for use with the beam-loss monitor electronics to alert operators when the amount of spilled beam was approaching the level at which the beam would be truncated. Software drivers for each of these devices were written, and the entire system was demonstrated successfully on experiment $2 \mathrm{~A}$.

\section{Optically Isolated Binary Output Module}

Some GTA injector reliability problems persisted as a result of electro-magnetic interference noise-induced failures of the Allen-Bradley modules used to control the high-voltage dome electronics. We determined that the AllenBradley contact output modules (Model OW) were the most likely cause of the problem. Consequently, replacement modules with high-electrical isolation were designed, built, and installed. No further reliability problems associated with injector controls were encountered.

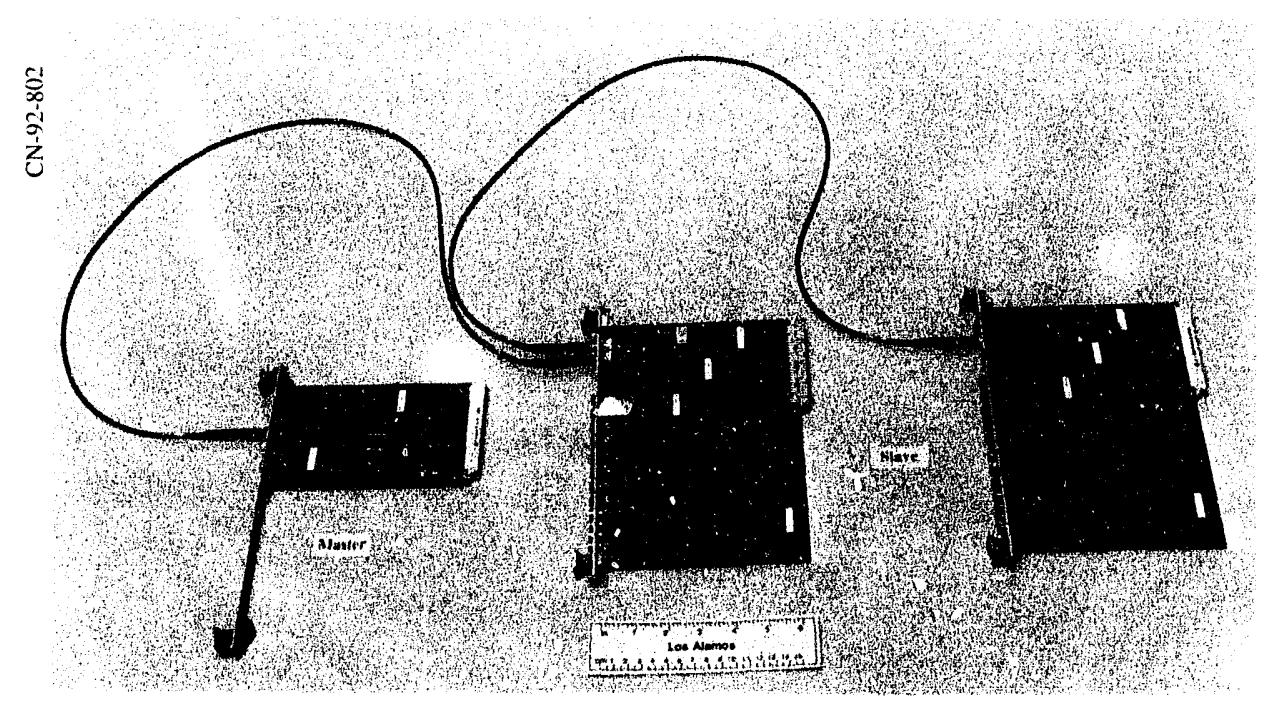

Fig. 7.1. Fast-Protect System.

\section{Step-Motor Control}

AT-8 incorporated a convenient scheme design into the existing motor driver backplane electronics for controlling brakes on step motor assemblies (e.g., the offset beamline harp assemblies and high-energy beam transport [HEBT] variable-field quadrupoles).

A new version of the motor driver backplane has been designed to support four motors with incremental encoders. This version will be required for HEBT motor control. A compatible VME transition board has also been designed.

\section{Transient Digitizer}

Transient digitizers with analog bandwidths of up to $10 \mathrm{MHz}$ are needed and the demand is expected to increase for experiment 2D. Currently, only the Joerger model VTR-1 transient recorders have been used routinely on GTA, and the 8-bit resolution of these units is not sufficient for several applications, including beam jitter and rf feedforward control studies. This year Omnibyte, Inc. began manufacturing a four-channel, $5 \mathrm{MS} / \mathrm{s}$, 12-bit module designed by FERMI Laboratory for a very attractive price. We purchased and evaluated one of these modules and concluded that although it suffers from digital noise pickup in the board's analog front end, which limits usable resolution to about 10 bits, the module was found to be an attractive alternative to the VTR-1 in most respects, including a much lower cost per-channel.

\section{Next Generation Computer for IOCs}

The Motorola MVME-167 central processing unit module was evaluated as an alternative for the aging Heurikon HKV2FA units we presently use. The 167 incorporates many important advantages including a 68040 processor, on-board ethernet interface, less heat susceptibility and larger EPROM and RAM capacity. The 167 processor runs control applications at 6 to 10 times the speec of the HKV2FA and costs about $25 \%$ less to field in a typical application. 
We purchased the support software for this new board and converted four test IOCs to use the 167 so that future control system applications can be developed and tested with the 167 instead of the HKV2FA.

\section{Binary Output}

The Xycom XY240 VME binary output module was thoroughly evaluated as a possible replacement for the XY220. The 240 was found to be a superior module and a recommended replacement for future applications. We wrote a software driver to allow this module to be incorporated into the EPICS control system.

\section{Nonvolatile IOC Memory Module}

A Force RR-2 VME memory module was fitted with $2 \mathrm{MB}$ of SRAM and an onboard battery for backup. A file system was created and the board was configured as a RAM disk. Such a configuration may be used for future applications that require a completely self-contained IOC running with no network resources or with operating parameters that must be maintained even if there is a power outage.

\section{Electronic Computer Aided Design (ECAD) Support}

The Electronic Computer Aided Design (ECAD) effort included supporting AT3's diagnostics design requirements, AT-5's low-level and high-power if design requirements, and AT- 8 's controls hardware development requirements. Most of this support effort was directed toward designing printed circuit boards and associated hardware to meet these requirements. In FY 1992, we placed 80 printed circuit board fabrication orders, amounting to more than 850 boards delivered.

ECAD also increased design efforts in the area of mechanical packaging and documentation. This included the layout and design of low-level VME EXtended INterface, (VXI) printed circuit board module, and submodule assemblies.
VXI mechanical module templates were developed in Autocad to aid in the documentation of each type.

We increased our extensive, customized parts library for printed circuit board design and our documentation for a variety of parts. This customized parts library ensures consistency in design among many ECAD designers and supplies useful and relevant information for the parts list extraction process. Parts lists extracted from each design include a parts description, reference designation, manufacturer's part number, and manufacturer's name.

\section{Computing Support}

The AT Division computing network supports exceptionally diverse computer equipment and applications. AT-8's computing support section is responsible for operating and maintaining the division's network. Using modern, but proven technology, this section strives to maintain high-quality network services in a heterogeneous computing environment containing over 150 work stations, over 150 personal computers (IBM and Macintosh), approximately $20 \mathrm{VAX}$ computers, and several network file servers. The services range across two open, unclassified networks and two classified networks. Extensive network equipment, including bridges, routers, and terminal servers, are used throughout the network, and network statistics are collected at a central location. The computing support section is also responsible for complying with various Laboratory and Department of Energy policies and orders for open and classified computing.

AT Division networks support a wide spectrum of scientific computing, including measurement and control systeins, software development, mechanical computer aided design (CAD), electronic CAD, experimental data reduction, and system modeling. Resources such as printers and plotters are shared among users, and the electronic mail services for all computers are integrated throughout the open network and with the Laboratory's Integrated Computing Network (ICN). Open standards-TCP/IP, DECNET, XWindows, NFS, NIS, and DNS for example--are used when possible. As in the past, the reliability of the network and availability of central services has steadily improved, consequently, AT-Division network is a model for others in the Laboratory.

During FY 1992, a major effort was made to consolidate computer services for the VAX computers and Sun workstations, resulting in reduced costs for support and maintenance, improved reliability, and increased performance. For instance, the original $6 \mathrm{VAX}$ clusters with $28 \mathrm{VAX}$ computers were consolidated into 3 clusters and 17 computers, resulting in an annual savings of approximately $\$ 50 \mathrm{~K}$ in hardware maintenance, while maintaining the same computer power. Similar upgrades to the Sun file servers on the open network resulted in comparable savings. We also added mail service and central file backup capability to the personal computers.

AT-8's computing support section surveyed the division members to determine the requirements for database management. From this survey, we determined that a client/server model relational database management system (RDBMS) would meet the division's requirements. We evaluated potential commercial RDBMS and made preliminary recommendations to the division. 

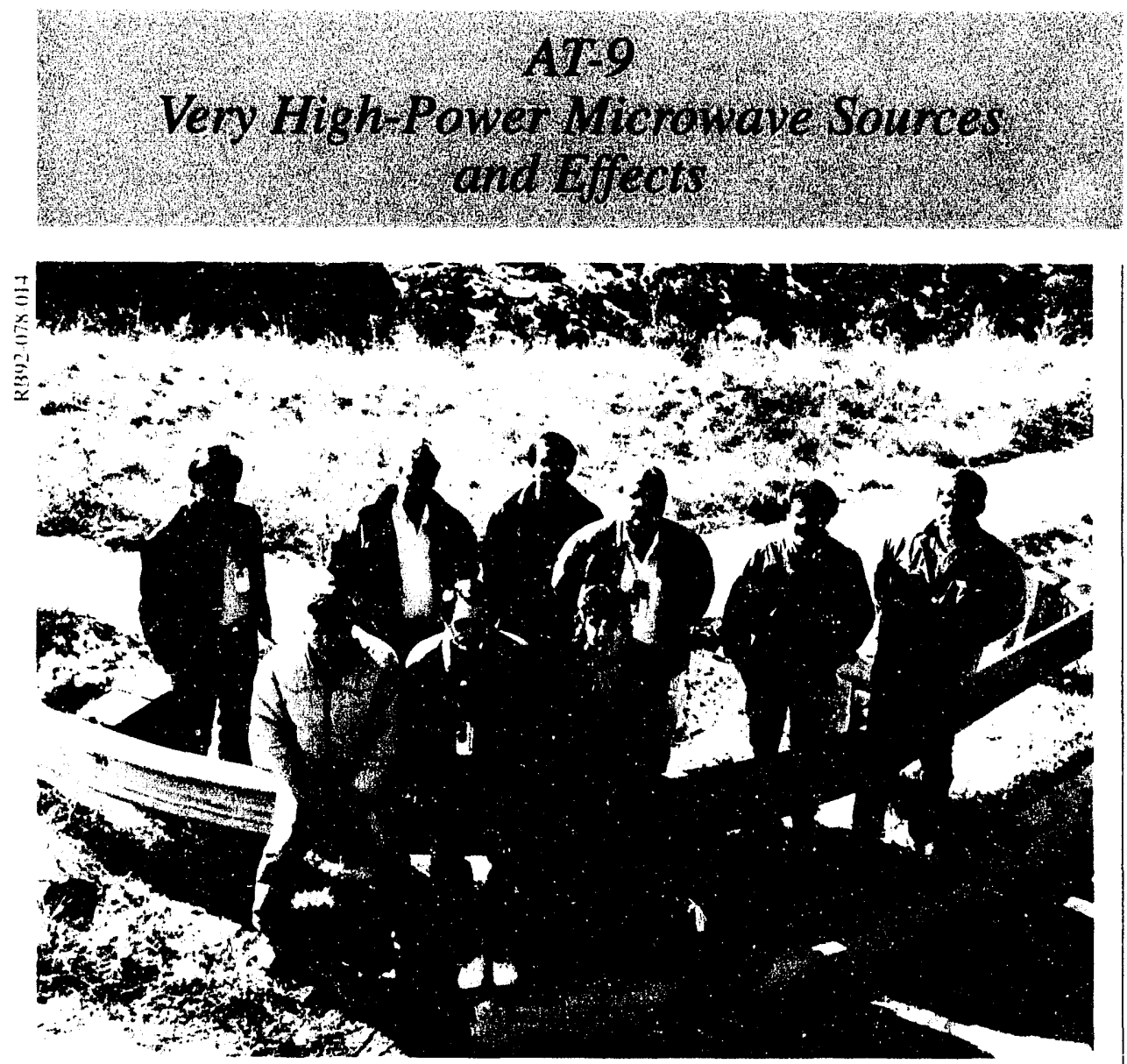

Introduction 9.3

Background 9.3

Microwane Source

Development 9.3

Relativistic Klistron

Amplifier... 9.3

Large Orbit Gyrotron .96

Piesoelectric Experiments 100

Pulsed-Power Research and

Development 102

BANSHEE 102

Portable Pulser. 103

WEMPE 4 10.3

Electron-Gun Test Stand 103

Vulnerability: Lethality; and Effects (VLE) Testing 10.3

High-Level Testing of Mobile Militury Sistems. 103

Test Point Impedance

Assessment 104

High-Performance Ground Penetrating Radar. (HIPERGPR) 10.5

Materials Processing with $\mathrm{HICH}$ POWER MICROWAVES (HPM) .... IO6

Wideband Antenna

Developme'nt 106

1.30()-MHz RF for the Advanced Free'Electron Laser. 106 


\section{Introduction}

AT-9 is the Very High-Power Microwave Sources and Effects Group within Los Alamos National Laboratory (LANL). The group's mission is to develop new high-power microwave (HPM) generators and apply them to Department of Energy (DOE) and Department of Defense (DoD) problems. The AT -9 efforts span the range from studying the basic physics of high-current electron beam propagation to the application of HPM to industry. The common theme among these projects is very high-peak power microwave radiation. These high peak powers lead to interesting physics as well as to challenging engineering in most of our work. The group's projects are divided into two broad categories with some overlap: microwave source technology development and microwave source technology application. Major applications for HPM are defense (electromagnetic countermeasures), radio frequency sources for particle accelerators such as the proposed Nexı Linear Collider (NLC), advanced radar, and industrial uses such as coal processing to minimize waste products.

\section{Background}

AT-9 was originally founded to investigate the defense implications and applications of HPM and transientpulse technology. From its inception. our group has had a two-pronged approach of investigating how to produce HPM with our microwavesource development work, as well as how to apply HPM technology. The applied work began with electronics vulnerability testing to quantify susceptibility against microwave radiation. This has evolved into an extensive test program, both at L.ANL and at large transient electromagnetic simulation facilities elsewhere. AT-9 has been involved in transient-pulse technology as well, developing transmilters, antennas, and diagnostics that have been used for radar and vulnerability testing.
Fiscal year 1992 has been successful for AT-9. One of our leams received the Laboratory"s Distinguished Performance award for experimentally testing and demonstrating a single-shot microwave-source concept in record time. We have made substantial progress on both the large-orbit gyroklystron and relativistic klystron amplifier (RKA) projects, with 400)MW output power measured in the RKA work. AT- 9 has worked to get the Advanced Free-Electron Laser (AFEL) on the air and has performed extensive Vulnerability, Lethality, and Effects (VLE) testing; new initiatives have begun on both defense and industrial projects. Funding for AT-9 activities is supplied by a variety of DOE and DoD sponsors. Within the DOE, our funding supports science (large-orbit gyrotron [LOG] and RKA), hardness testing, cleanup (ground penetrating radar (GPR) and microwave coal beneficiation), and participation in the AFEL. The DoD sponsors include the Army, Air Force and Navy.

\section{Microwave Source Development}

The NLC is a proposed linear accelerator that is to be built after the Superconducting Super Collider (SSC) is completed. This machine will allow energetic electron collisions to be studied at much higher energies than currently possible at Stanford Linear Collider (SLC) and LEP at CERN. The accelerator will require many gigawatts of peak microwave power to accelerate the particles. To minimize cost, the rf power sources must be of very high peak power with high frequency $(1-20 \mathrm{GHz})$ and high reliability. These ambitious goals require major advances in microwave tube technology rataging from the electron gun and cathode . . . to the optics of intense space-charged beams ... to the nonlincar interactions involved in bunching the beam, to the high-power output couplers and windows.
The military requirement for HPM remains strong even after the recent changes in the world military picture. Sophisticated, sensitive electronics are pervasive on the modern battlefield. HPM has a role in the give-and-take between these electronics and the countermeasures employed against them. The defense reyuirements for HPM sources encompass the NLC needs, but are more diverse. Some defense applications are single shot, others are tunable, while others may be transient, wideband pulses. AT-9 is currently investigating the LOG and the RKA. Each source concept has strengths that make it useful for hoth scientific and military application.

\section{Relativistic Klystron Amplifier}

Los Alamos is developing an L-band high-current RKA. Although present experiments are single pulse, the longterm goal is to achieve $1 \mathrm{~kJ} /$ pulse with repetitive pulse capability at a repetition rate of $5 \mathrm{~Hz}$ with a longer-term goal of $100 \mathrm{~Hz}$. The RKA has an input cavity, a single idler cavity, and an outpul cavity (Fig. 8.1). The buncher section, which consists of the input and idler cavities, has been experimentally tested and is performing as designed. We designed the buncher section by using particle-in-cell (PIC) code calculations with the Los Alamos code ISIS. PIC code modeling has proved to be very important for suceessful design because of the highly nonlinear nature of the RKA calused by the intense space-charge effects. The most recent efforts involved adding the output cavity to the tube and experimentally optimizing the output power and pulse length to reach the design goal of $1 \mathrm{~kJ} /$ pulse. A number of expected problems that have been encountered with the output cavity are being systematically addressed. Problems include rl breakdown in the cavity and the ability to match the beam impedance to cavity gap shunt impedance for the most efficient conversion of beam power to microwave radiation. 
The electron beam is formed from a 6.2-cm-diameter annular field-emission cathode and is slightly compressed by a converging $0.5 \mathrm{~T}$ axial magnetic field to a nominally 5.9-cm-diameter beam with a 5 -mm thickness. The typical beam voltage is $620 \mathrm{kV}$ with an increasing current of 3-6 kA. The input and idler cavitics are quarter-wave coaxial resomators. The input cavity is coupled to a $30(0)-k W$ L-band magnetron through an iris into a tapered, reduced-height WR-6.50 waveguide. The idler cavity has an annular tuning ring which gives flexibility in induclively tuning the cavity. The output cavity, a noseless pillbox resonator with an annular coupling slot near the outer diameter. couples power into a low-impedance coaxial transmission line. The coax tapers out into standard 6 inch 50 line in which power is measured with a directional coupler and then dissipated in a dummy load.

The input cavity was changed from a loop coupling design in the original RKA to a waveguide input with iris coupling to accommodate the increase in drive power from $5 \mathrm{~kW}$ to a maximum of $300 \mathrm{~kW}$ (Fig. 8.2).

The coupling iris is large enough for the input cavity to behave as a matched load when the beam is present in the gap. Consequently, the cavity is very mismatched and reflects most of the incident rf power before the beam current turns on. Then as the beam traverses the gap, the cavity is nearly matched, and most of the magnetron drive power is absorbed and transferred to modulate the beam. We confirmed the low reflection with beam loading by computer modeling. The comparison between theory and experiment is quite good and is shown in Fig. 8.3.

According to PIC code calculations, the amount of current modulation produced by the input cavity, necessary for fullpower output of the tube, was about 10\%. As shown in Fig. 8.4, the input cavity is easily capable of producing $10 \%$ modulation with only $200 \mathrm{~kW}$ of injected rf power.

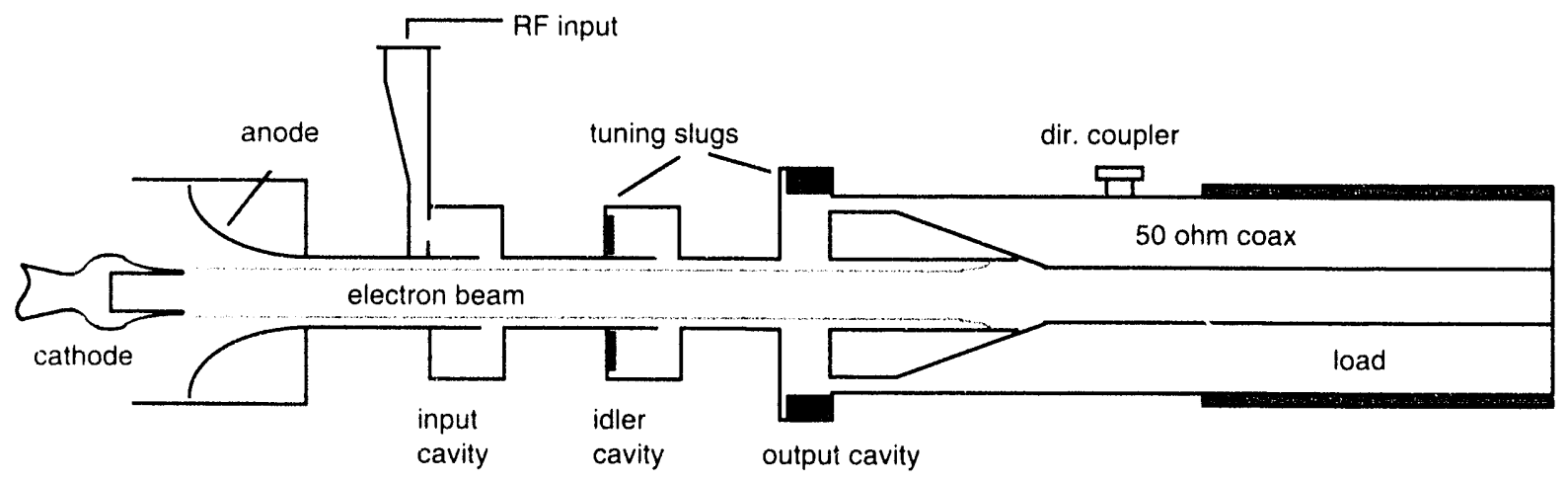

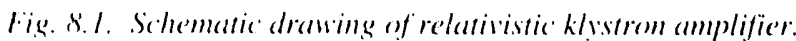

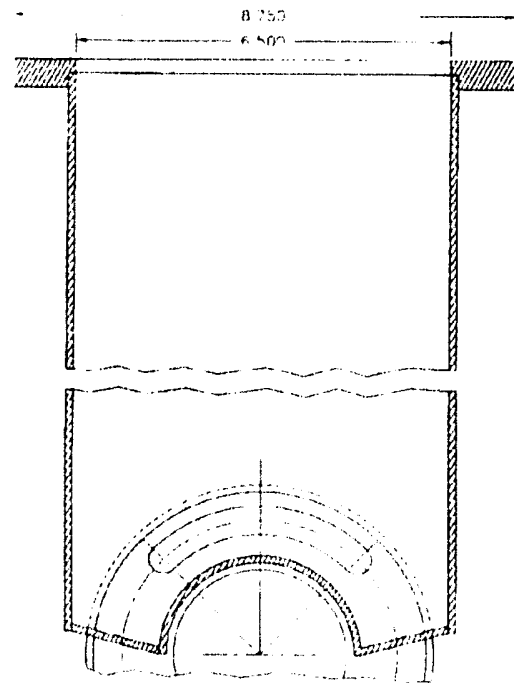

Fig. S.2. RKA inpun cority design.

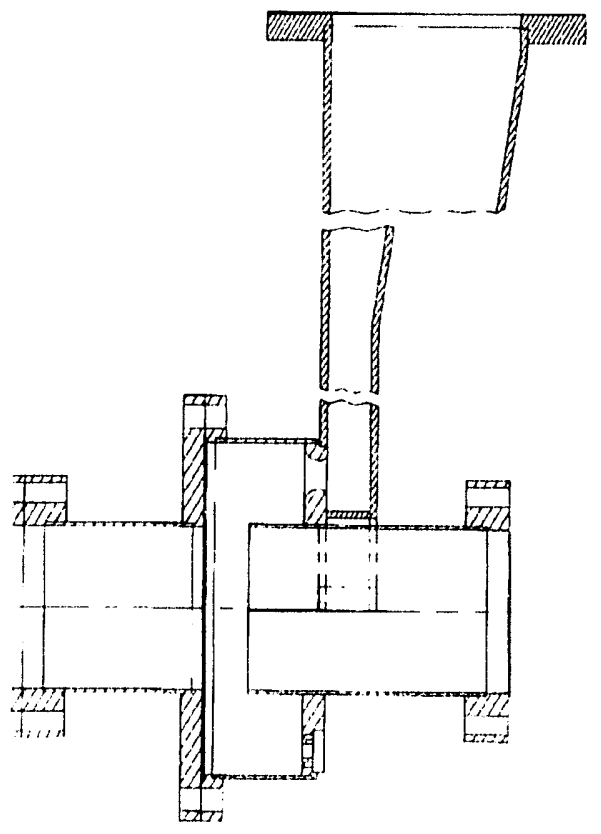

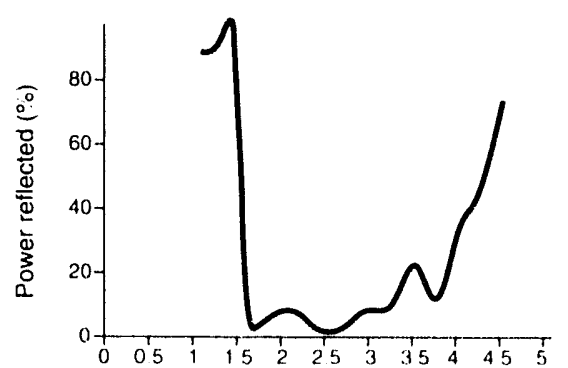

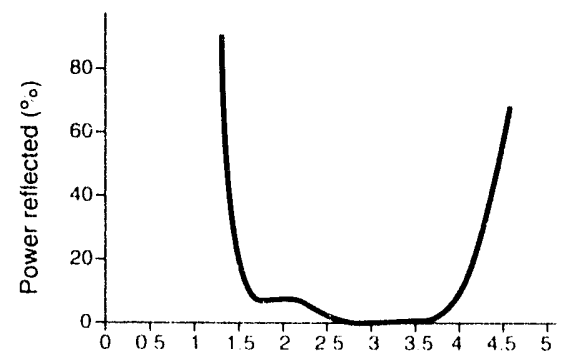

Fig. S..3. Theoretical laner) and

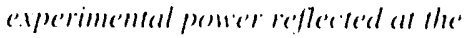
iilpul cority: 
We modified the original RKA idler cavity to include a tuning ring so the cavity could be inductively tuned (the cavity's resonant frequency is higher than the modulation frequency). This allowed for greater beam current modulation at the output of the idler cavity for the same amount of bunched beam at the input. Once the cavity was installed on the beamline, data were taken for different tuning frequencies and input cavity drive powers to find the best combination for maximum beam modulation. Figure 8.5 shows the good input match during the pulse, as well as an output power of more than $100 \mathrm{MW}$.

Using PIC code simulations, we determined the maximum output of modulated current from the idler cavity to be $65 \%$ to $75 \%$. Figure 8.5 shows the good input match during the pulse, as well as an output power of more than $100 \mathrm{MW}$. The average beam modulation is near $66 \%$ over the pulse length. The amount of extractable beam power is usually given as $P=\left(V \cdot I_{1}\right) / 2$. For this case, about $1 \mathrm{GW}$ of extractable energy is available at the fundamental frequency. The output cavity should be at least $70 \%$ efficient and should produce $700 \mathrm{MW}$ of if power out or $700 \mathrm{~J}$ per pulse.
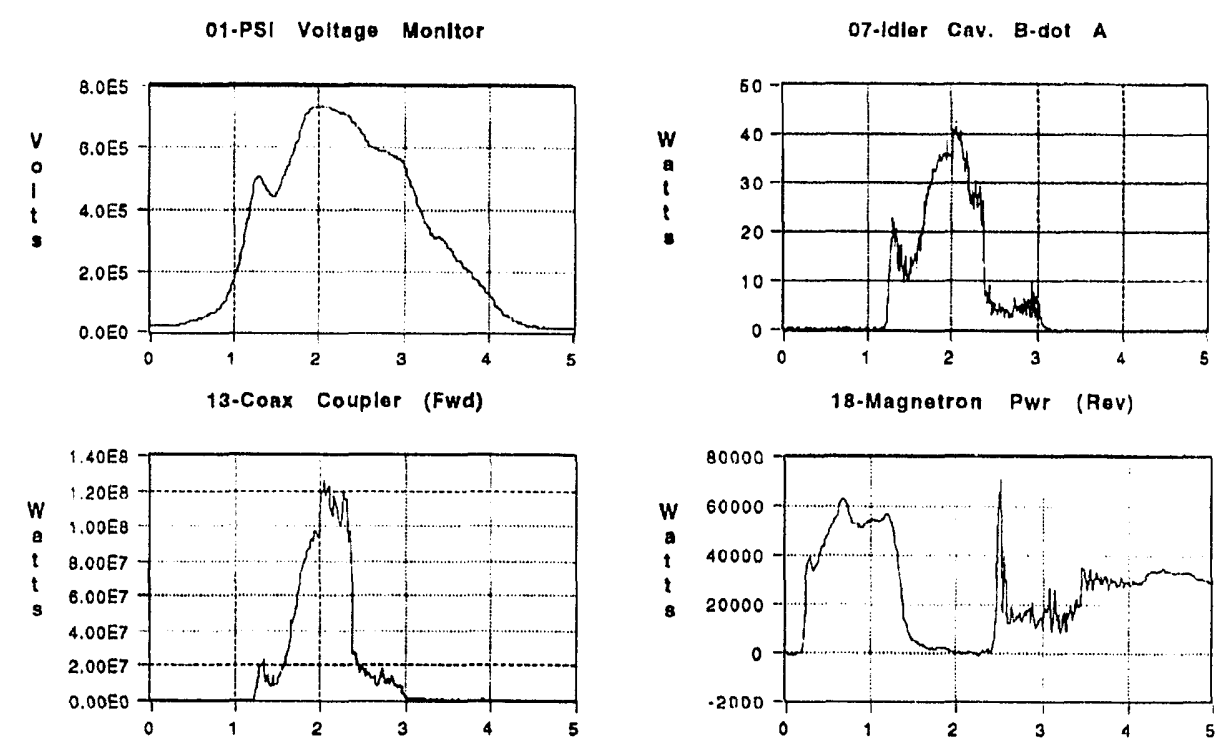

Fig. 8.5. Data for Shot \#1196 showing good match of input, as well as $>100-\mathrm{MW}$ output power.

Power from the initial output cavity design was significantly less than expected. One difficulty with the modeling was that a $2 \mathrm{D}$ code was used to calculate the interaction, which does not realistically model the three dimensional geometry of the actual experiment. Even though the designed $\mathrm{Q}$ of the cavity was 10 , the measured cavity $Q$ was approximately 200 and therefore could not be tuned on resonance for maximum efficiency without producing electric fields that exceeded the level for $r f$ breakdown.

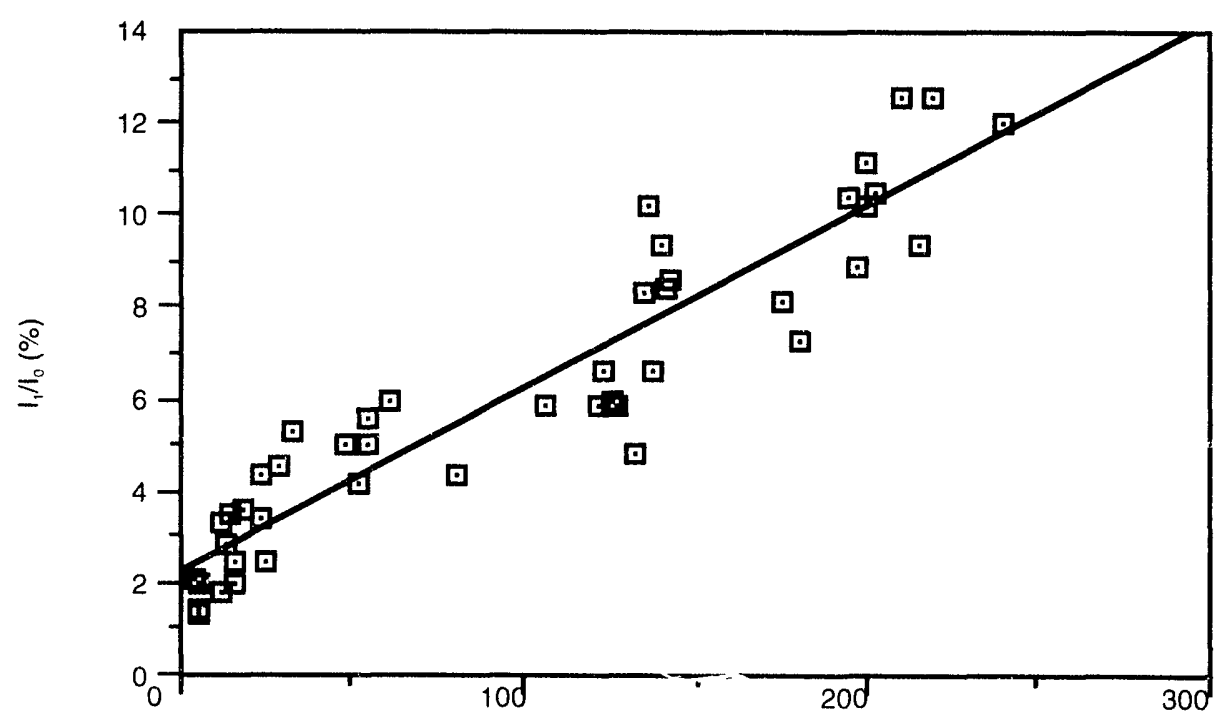

Magnetron Power (kW)

Fig 8.4. Current modulation downstream of input cavity as a fimction of ef input power. 
The first mode is one in which not much power is produced even though most of the waveforms are essentially good. The idler cavity signal is taken from a B-dot in the cavity. As can be seen in Fig. 8.6, the cavity signal stays at a relatively low value throughout the pulse for no apparent reason. Output power is usually in the range of 2010 40) MW for $0.5 \mu \mathrm{s}$.
In the second mode, the idler cavity shows reasonable modulation at the very begimning, but then lalls off abruptly after about 300 ns. This waveform ustailly correlates with the beam voltage changes at the heginning of electron emission from the callode. but an initial bump in the beam voltage does not always produce the same problem in the idler. Output power typically spikes to about 20 to $50 \mathrm{MW}$.

\section{1-PSI Voltage Monitor}

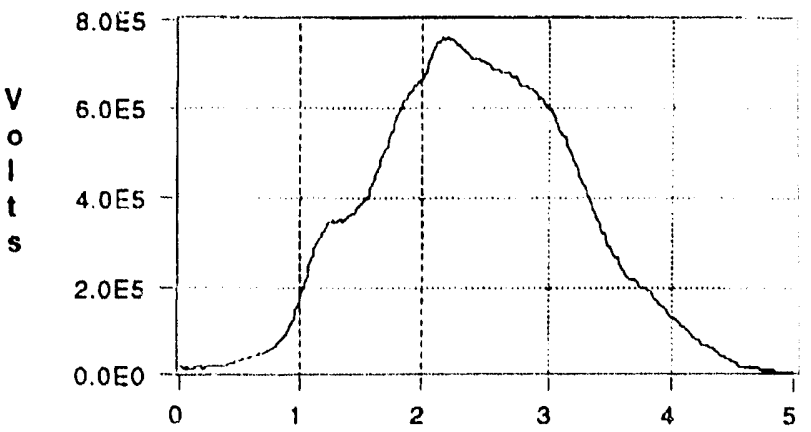

07-Idler Cav. B-dot A

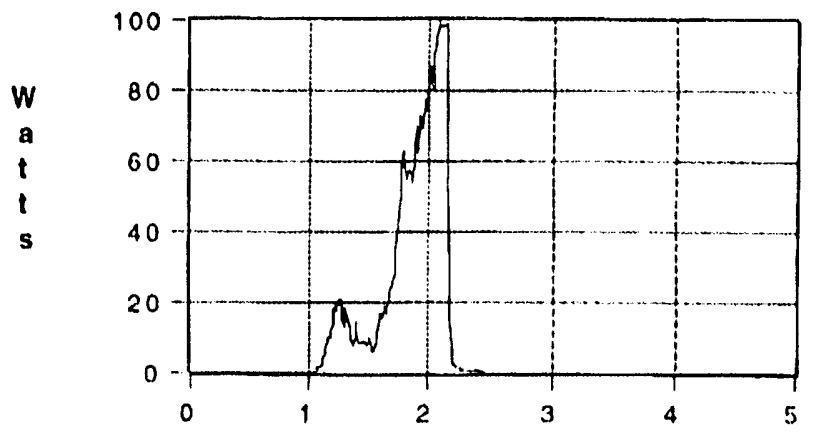

13-Coax Coupler (Fwd)

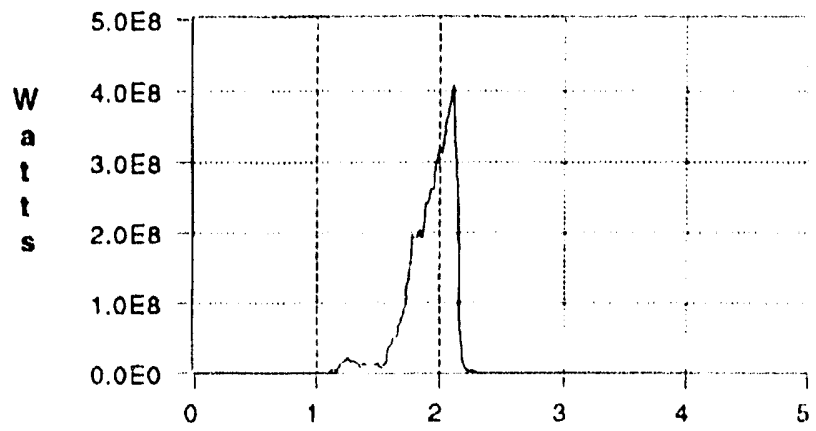

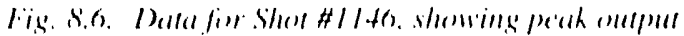

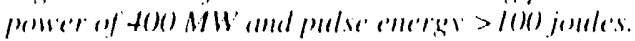

The third mode consists of the idler cavily modulating well for the first half of the pulse. but then something seems to change the beam modulation at the inpul cavity. This, in lurn, causes a change in the inpul cavity match and increases the amount of reflected injection power. With the drive power to the inpul cavity dropping. the desired beam modulation also drops off and drops the output power. Often, the begimning of the idler cavity drop correlates with the begimning of lhe beam vollatge's descent. Output power is reasomable in these shots and very offen firom 8() $101.30 \mathrm{MW}$ for $0.5 \mu \mathrm{s}$.

In the fourth and linal mode. the idler cavity works well. The mulpul power climbs to the point where the output cavily galp ficld is high enough to reflect electroms back up) strcallu. Comseguently, the idler cavily breaks down and rends the pulse, usually (mily 3.50 ) $1 \mathrm{~s}$ from the initial start. Average outpult power ranges from 200 to $300 \mathrm{MW}$ over the ramp-shaped pulse with peak power levels as high ats $4(0) \mathrm{MW}$. The gain in this mode is about 36 dB. In the main part of the pulse, there are $11(0$.$) of if energy.$

If the fourth mode could be chosen selectively, the breakdown might be solved by lowering further the output cavity $Q$, thus reducing the gap fields and also modifying the idler cavity to handle higher tields without breaking down. However, only the third mode gives output powers that ate consislent with the gains meatsured all low power injections. Solving this problem is difficult, becaltse there are no data fo correlate directly with the problem to datle. The breakdown could be a result of the changing beam voltage affecting the waly the inpul cavity bunches. Alternatively, higher beam harmonics could he propagating up the beam pipe creating the mismatch in the inpul cavity.

\section{Large Orbit (jyrotron}

We hatve designed and are lesting a L.OG amplifier for 1.3 (illz operation in 6.5 ns pulses. The ultimale power output goal is $500 \mathrm{MW}$ with a gain of at least 20 dB. This initial investigattion is intended to lay the groundwork for operation all 11.4 GHz lor particle acceleralor applicaltoms and also al frequencies of up to 3.5 (ittz for other uses. Computational design has been performed with the resomant cavity code MAIIA and the PIC codes MIERI.IN and ISIS. Lxperimental measurements of the resomallor modes were correlated with computalional and analytical predictions. We studied and experimentally verified electron-beam optices through a magnetic cusp with ISIS and MI:RI.IN Io develop a suitable clectrom-beam majectory from the electron gun into the microwate resomator region. Performance tests have hegun. In its final form, the device will use two resemallors sepatralled by ant electron-beam drilt lube 
A LOG amplifier operating at $1.3 \mathrm{GHz}$ is being developed to operate at powers of up to $500 \mathrm{MW}$ for $65 \mathrm{~ns}$ pulses. While this initial investigation is being performed at $1.3 \mathrm{GHz}$, this amplifier can be scaled to higher frequencies in a straightforward fashion. LOG oscillators have operated at $15 \mathrm{GHz}$ and higher frequencies with comparable performance at lower frequencies. Amplifier operation has been examined theoretically and experimentally, but less extensively.

These LOG amplifiers produce microwaves when a helically rotating electron beam interacts with the oscillating fields of a resonant cavity structure. The electron beam is formed by injecting a hollow, nonrotating beam, born in an axial magnetic field, through a magnetic cusp positioned at the anode plane. The gyrotron radius of the electron is equal to the radius of the annular beam, so this device is referred to as "large-orbit," as opposed to a conventional gyrotron in which the orbits may be very small because of a relatively high magnetic field. An annular slot is cut into the iron cusp plate to allow the beam to pass into the downstream resonator. In the cusp, a portion of the axial beam energy is converted to rotational energy. Typical ratios of rotational velocity to axial velocity (defined as $\alpha$ ) are in the range of 1.5 to 2.5. The electron beam entering the resonator has an energy of 500 to $700 \mathrm{keV}$, a current of 1 to $3 \mathrm{kA}$, and a radius of 5 to $8 \mathrm{~cm}$. The device, shown in Fig. 8.7, employs a cylindrical resonator with three vanes in the wall spaced equally in azimuth.

The amplifier is designed to have two cylindrical resonators as described above. The vane structure is used to evoke coupling of the rotating electron beam with the transverse electric $\operatorname{TE}(0,1, n)$ resonant cavity mode of the cylindrical structure by modifying the normally circular electric field pattern of the mode into a scalloped pattern, similar to the $\operatorname{TE}(3,1, n)$ mode but near the lower $\operatorname{TE}(0,1, n)$ resonant frequency for a nonvaned cylindrical wall with an intermediate radius.
We used two loops, one in each of the two vanes, to feed if into the cavity. If the beam's angular velocity is synchronized, the cavity's standing wave pattern will couple to the rotating beam provided. Consequently, an azimuthal density perturbation will grow on the beam with three-density maxima around the azimuth. The magnitude of the density variation will grow as the beam propagates down the resonator's length, influenced by the applied oscillating rf fields and the space-charge self-fields that drive the negative mass instability. The instability will grow as the electron beam propagates through the system. Feedback from the beam instability drives the cavity fields to greater amplitude.

The first cavity's downstream end has a central opening that forms the entrance to a cylindrical, nonvaned electronbeam drift pipe. The pipe isolates the rf radiation between the first and second cavities and serves as a region where the beam bunching can grow by the negative mass instability, independent of applied microwave fields. An optimum drift pipe length will be determined experimentally to maximize azimuthal beam bunch:- g. To extract energy, a second output resonator designed to be strongly coupled to the beam will be placed downstream of the drift pipe at the point of optimum beam bunching. Mode converters suitable for transforming the TE01 circular waveguide mode of the output resonator into TE10 rectangular waveguide mode have been thoroughly studied since the 1950s.

We designed the electron gun and optimized the eleci"on beam trajectory using the two-dimensional versions of ISIS and MERLIN PIC codes. 'Preliminary studies were performed with a computational technique known as synthesis. This technique steps the particies backward in position and time from the final state, (i.e., beam current, position, and velocity components). The initial conditions (i.e., emissionelectrode position, shape, and potential) that lead to the final state, and a satisfactory trajectory through the device can be determined.

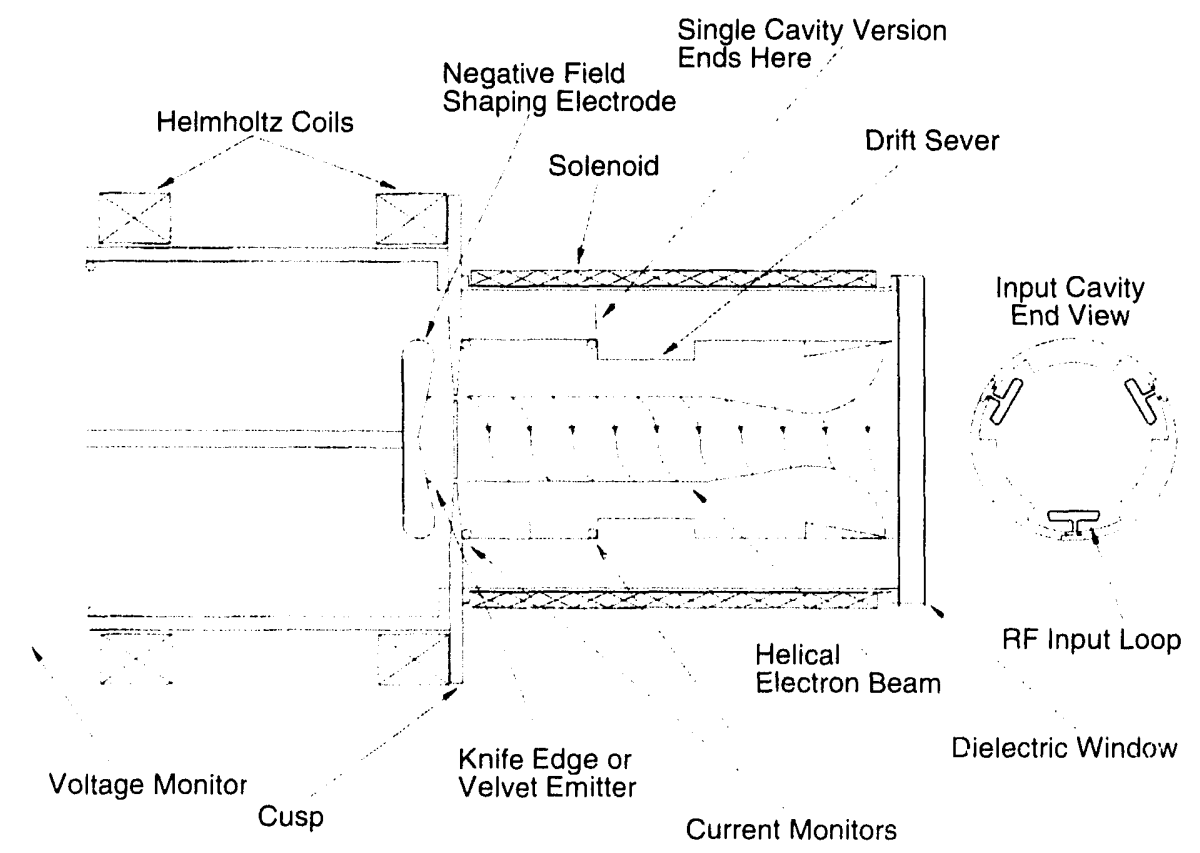

Fig. 8.7. Schematic drawing of large-orbit gyroklystron amplifier. 
An acceptable synthetically generated diode configuration consists of a cathode-emission annulus with a diameter of 14 to $14.2 \mathrm{~cm}$ on a conical equipotential surface at an angle of $67.5^{\circ}$ with respect to the symmetry axis (Fig. 8.8). The distance between the emission annulus and the anode was 2.2 $\mathrm{cm}$, yielding a cathode electric field of $300 \mathrm{kV} / \mathrm{cm}$ at a voltage of $650 \mathrm{kV}$. The annular opening at the anode through which the beam passes into the resonator drift section has a mean diameter of $12.5 \mathrm{~cm}$ and a width of $1 \mathrm{~cm}$.

We began with a synthetic computation that was the starting point to conventionally calculate beam dynamics moving forward in time. A conventional run using the diode and drift-pipe parameters from the synthetic calculations agreed with the synthetic calculation prediction. All of the current emitted from the cathode, up to a maximum of $2.7 \mathrm{kA}$ passed through the cusp and drifted with little radial oscillation while in the resonator region.

We built the diode hardware using PIC modeling; the experimental diode geometry is shown in Fig. 8.9. Electron emission was produced from an annular region of the angled surface either by mounting a knife-edged ring or a velvet belt on the surface as an explosive emission cathode. The emitting ring's diameter varied from 11.4 to 14 $\mathrm{cm}$. The drift pipe had no vane structure for these diode experiments, to approximate the conditions of the computer model.

\section{Alpha, $(\alpha)$ was mea-} sured using a quartz witness plate placed in the beam path. The quartz's luminosity permits a pattern of the electron deposition to be seen at the plate location. A shadow is cast on the quartz when a metal rectangle is attached upright on

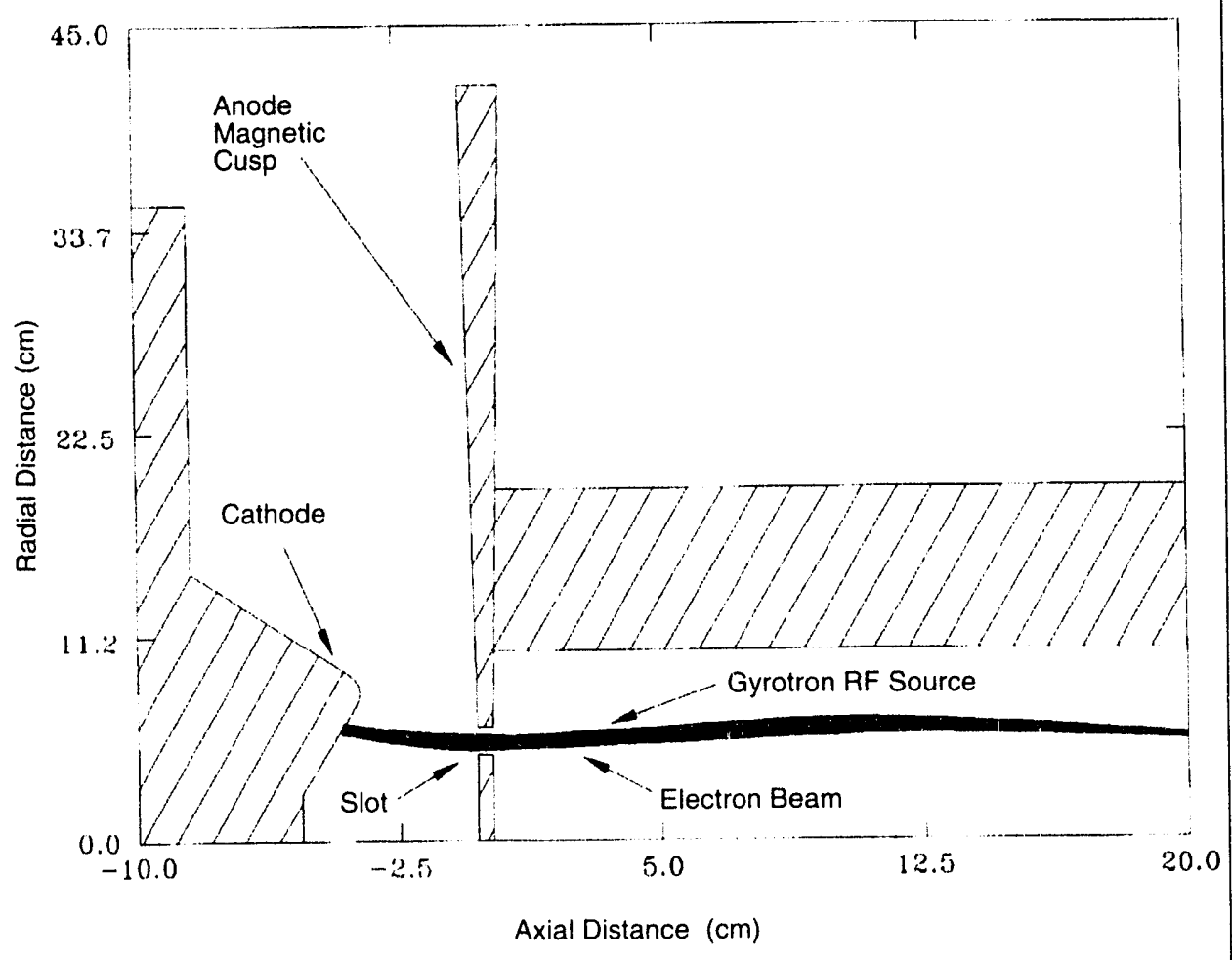

Fig. 8.8. Electron gun and beam optic's synthesized using computer modeling.

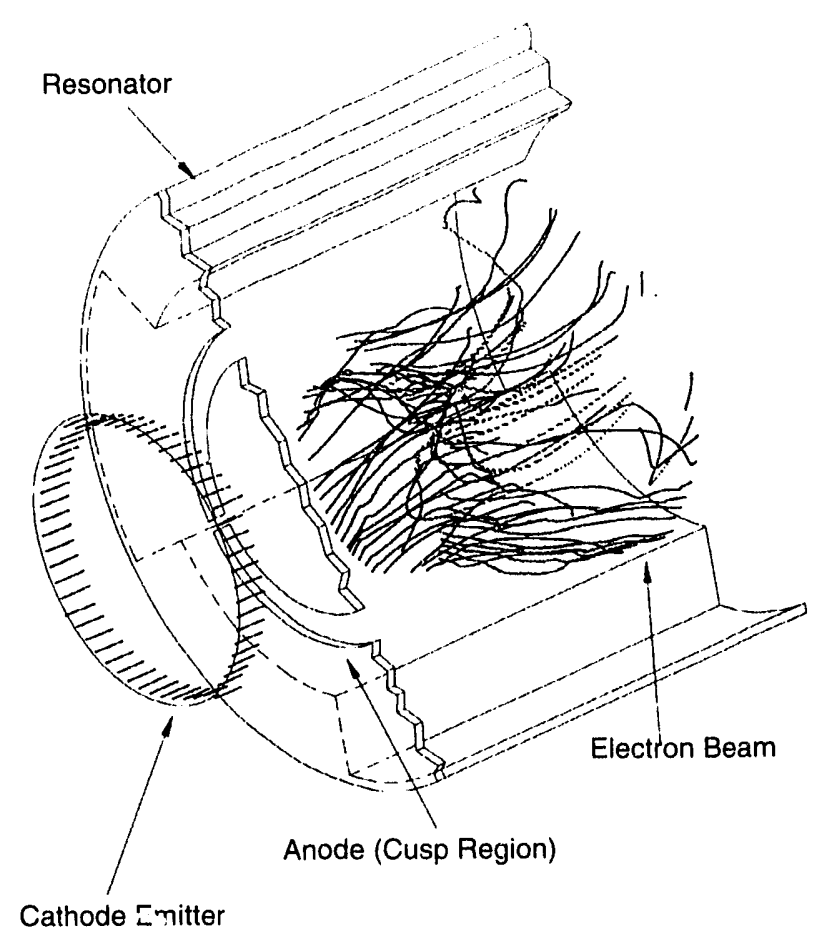

Fig. 8.9. Three-dimensional ISIS calculation of electron trajectories including dc and ac fields.

the upstream side of the quartz at the radius of the beam The ratio of the length of the shadow to the height of the obstruction is a measurement of $\alpha$.

Alpha was measured to be in the range of 1.5 to 2.0 for a magnetic field of $400 \mathrm{G}$ and in the range of 2.0 to 2.5 with a magnetic field strength of $500 \mathrm{G}$. This alpha range most accurately matched that shown by the computer model as providing good beam transport.

We transported $3 \mathrm{kA}$ past the cusp with an alpha of 1.5 using a knife-edge emitter with a diameter of $12.4 \mathrm{~cm}$, a magnetic field of 300 to $400 \mathrm{G}$ and a diode voltage of $700 \mathrm{kV}$. Waveforms for this case are shown in Fig. 8.10. These lower magnetic field conditions are those anticipated for the $1.3 \mathrm{GHz}$ amplifier experiments. The computer study did not include these parameters in its investigation, but rather used magnetic field of 500$) \mathrm{G}$ at the cathode, based upon the needs of an amplifier designed for higher frequency. 
We conducted an extensive study of the resonant structure's cavity modes using the electromagnetic fieldsolving code MAFIA, analytic modeling, and cold-test measurements performed with a vector network analyzer. Figure 8.11 shows the frequencies measured with a network analyzer connected to magnetic loop probes in the cavity, oriented to couple to the $\operatorname{TE}(0,1, n)$ modes. The peak of interest, the $\operatorname{TE}(0,1,0.5)$ mode, oscillates at $1,280 \mathrm{~Hz}$. Figure 8.12 shows the MAFIA calculated electricfield pattern for this mode at 1277 $\mathrm{MHz}$, showing good agreement with the cold-test measurement. When we test the input cavity with the downstream end fully open, the cavity had a $\mathrm{Q}$ of 45. As a two-stage device, the first stage $Q$ will be much higher, of the order of several hundred. The output cavity Q of the two-stage device will be similar to that of the single-stage resonator, in the range of 25 to 100 .

The experimental configuration consists of the diode, magnetic field coils, downstream resonator section, and a dielectric vacuum window. The vacuum window is used for radiating the microwaves into an anechoic volume downstream of the vacuum chamber. A 4-kW 1.3-GHz source provides $\mathrm{rf}$ input drive to the resonator in these initial studies. Up to $20 \mathrm{MW}$ is available as input drive, but device modifications are required for aperture coupling to accommodate the power. Presently, magnetic loops are located at the base of two vanes for cavity input drive. A loop in the third vane is used to monitor the cavity's standingwave field. A stub-waveguide receiver is positioned in the anechoic volume, downstream of the opened end of the resonator to monitor the radiated power.
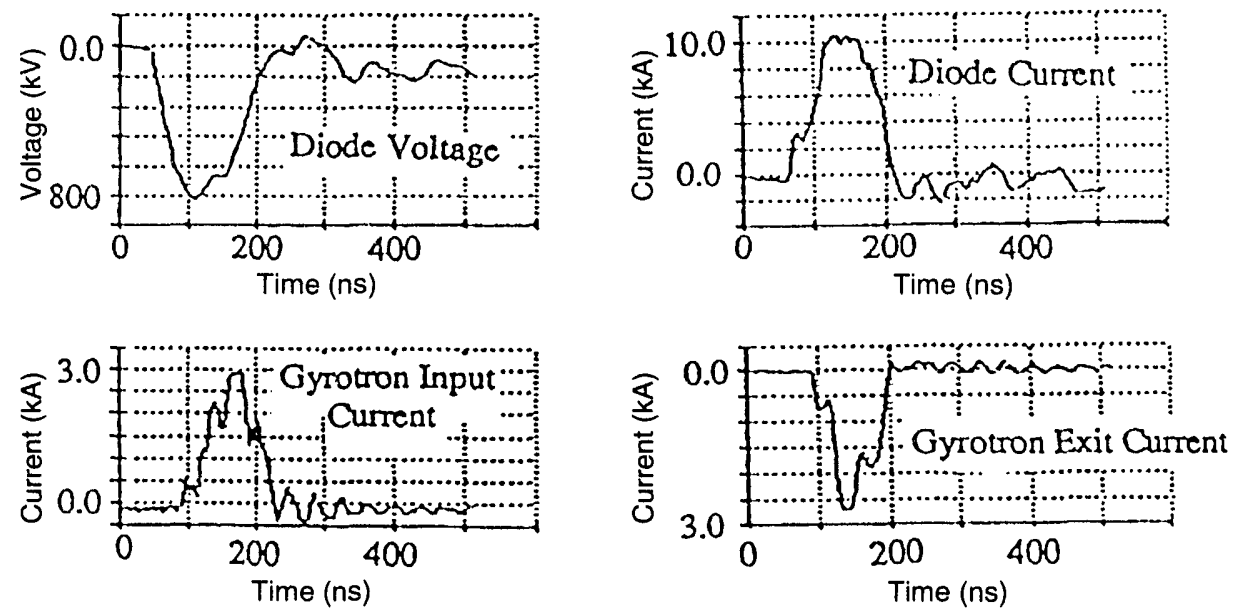

Fig. 8.10. Beam voltage and current from a typical gyrotron shot. Input beam power to gyrotron is $2.4 \mathrm{GW}$.

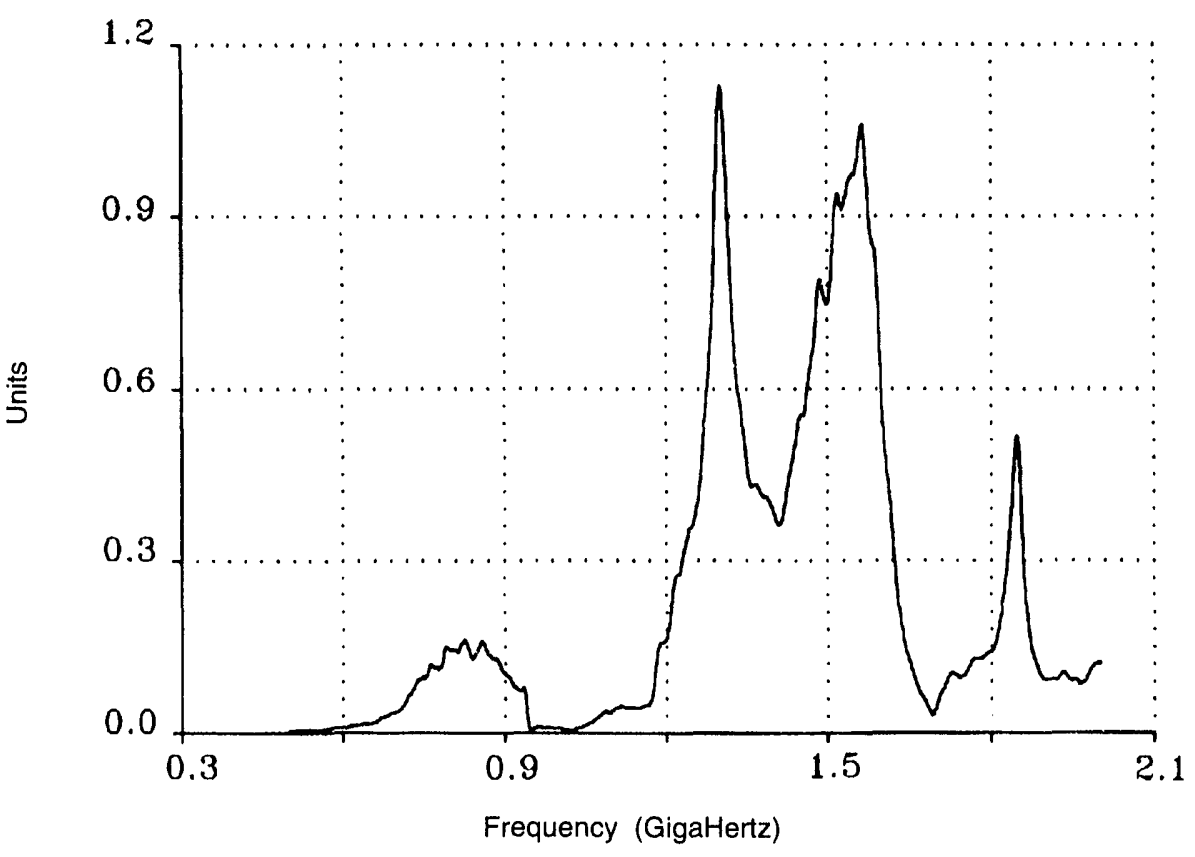

Fig. 8.11. Measured mode spectrum of gyrotron resonant structure. The strong peak at $1286 \mathrm{MHz}$ is the desired operating point.
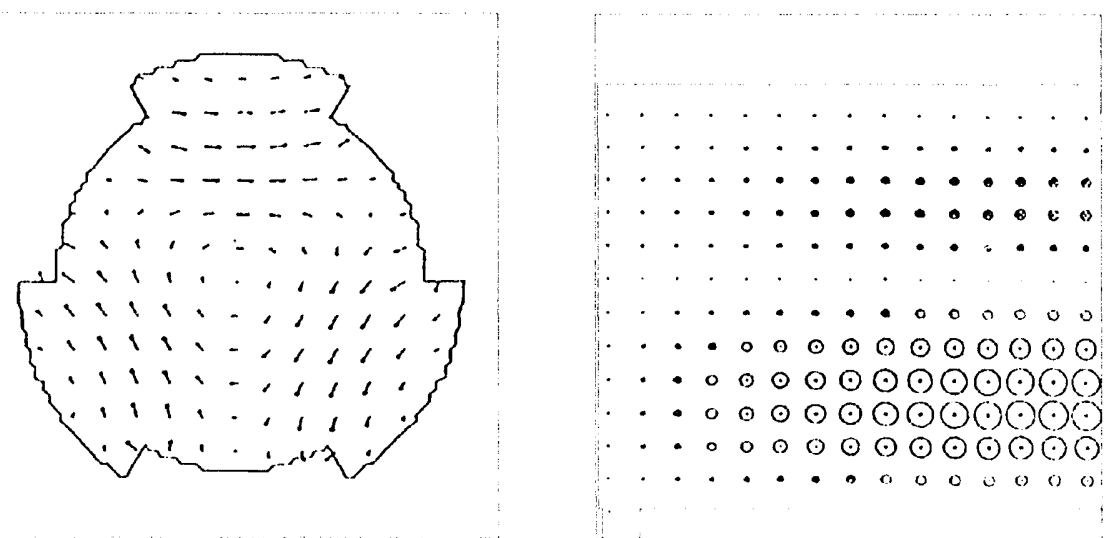

Fig. 8. 12. MAFIA output showing electric field distribution of 3-field symmetric mode at $1277 \mathrm{MHz}$. 
We evaluate amplifier performance by comparing the radiated microwave power in three different circumstances. First, the radiated power resulting from the $4-k W$ input drive alone is meatsured. Second, we measure the radiated power with no input drive, but with the electron beam injected. Finally, the radiated power is meisured when both input of drive and injected electron beam are present in the resonator. Relative power measurements among shots are performed by comparing detected signals received with a warveguide stub placed at a fixed location in the far field downstream of the resomaltor.

With no inpul drive, the raddiated if power pulse was narrow in time, 42-ns long. with a wide statistical standard deviattion of $24 \mathrm{~ns}$ or $56 \%$. This duration is significantly less (approximately $6.5 \mathrm{~ns}$ ) than the full width at half maximum (FWHM) duration of the clectron-beam current in the resonator. In addition. the mean value of the peak power, while larger than with input drive, had a $28 \%$ standard deviation. With input drive, the output power pulse continued for 60 ns, nearly the length of the electron beam pulse, with a standard deviation of $16 \mathrm{~ns}$ or $27 \%$. The peak power of the output pulse using input drive wals $26 \%$ lower than without input drive. but was longer by $50 \%$ with a smaller standard deviation of $16 \mathrm{~ns}$ or $27 \%$. The FWHM of the fast Fourier transform of the rf signal wals $36 \mathrm{MH} / 2.5 \mathrm{MH} /$ standard deviation) without input drive and 27 $\mathrm{MH} /(17 \mathrm{MH}$ \% standard deviation) with input drive. Hence, there was clear improvement in the output power pulse associated with the application of input rf drive. as was shown in total energy of the output pulse, the freguency purity, and in reduction of the statistical variation among pulses.

\section{Piezoelectric Experiments}

Compact sources of high-intensity electromagnetic radiation offer important advantages in counter-electronic military applications. At DoD's request, AT-9 and several other Laboratory groups developed a new concept and conducted simple, proofof -principle (POP) field experiments for producing a compact source of intense microwave radiation. These experiments demonstrated that microwave radiation is produced from pieroclectric material when it is compressed by at high-explosivegenerated shock wave. This is the first time microwave radiation has been produced directly from a high explosive without the complex, intermediate stage, which consists of a magnetic tlux compression-generator power supply that poduces a high-intensity electron heam, which is subsequently converted 10 microwaves. This entire effort from concept through explosive lesting was conducted in approximately 2 weeks. The group received the Laboratory's Distinguished Performance Award in recognition of its efforts.

Explosively-driven microwave and ultra wideband generators can produce mission kill of electronic equipment at stand-off ranges, preventing blast damage (or combined explosive and electromagnetic effects at choser ranges if desired). These systems can be delivered to close range by existing missile and antillery systems. Explosively-powered devices would enable much smaller payloads to exist, requiring much smaller delivery systems, thereby allowing a much wider range of uses.

A piezoclectric material becomes electrically polarized when mechanically deformed, or it changes dimension when placed in an electric field. Historically these materials hatve been erystalline such as quarty or ceramic. However. recent developments with organic polymers have resuled in materials with far greatler piesu activity that any other synthetic or natural polymer. This newer material is a flexible, compliant, ckear plastic film that can be readdly cut, shaped, and metalized with a conductive electrode coating. The properties of interest are - wide frequency range-near de lo low Cills.

- vast dynamic-range-sensitive to both minute forces and explosive shocks (nanobar to megahar)

- high-output vollage-10 times higher than piezoceramic for the same input forco

- high-de diclectric strength-75 $V / \mu m$

- high-mechamical strength and impact resistance

- very low raw material and fabrication costs

- reasonable dielectric constiant (relative dielectric constant $~ 12$ ) enabling a high stored energy

One characteristic of a high-explosive detonation is the intense shock wave that generates very high pressures in the 300$) 10.50(0)$ kbar range and as high as I Mbar in a convergent lens system. The high pressure in conjunction with a pic\%olectric material enables large voltages and currents to be generated. Picroelectric plastic film can produce electric fields of $0.3 \mathrm{~V} / \mathrm{m}$ per Pascal. Because ().1 Mhar $\left(=10^{111} P_{i 1}\right)$ is cassily achievable with a high-explosive detonation, we can generate electric fields of $3 \times 10^{\prime \prime} \mathrm{V} / \mathrm{m}$ assuming linear induced vollage vs pressure behavior of the material. This is a reasonable atsumption halsed on the manufacturer's specifications and on Sandia's work with piemelectric material in an unrelated application. Electric fields of, $3 \times 10^{\prime \prime} \mathrm{V} / \mathrm{m}$ exceed the de dielectric strength, which is about $10^{k} \mathrm{~V} / \mathrm{m}$. It is well known that as pulse length decreases the dielectric breakdown level increases, so thall als the pulse length is reduced from de to fractions of a microsecond, the breakdown level will increase significantly above the de dielectric strength of 1 lo $^{x}$ $\mathrm{V} / \mathrm{m}$. For comparison. the clectric fiedd produced by nuclear electromagnetic pulse (IEMP) is 10 - $\mathrm{V} / \mathrm{m}$. The pie/()electric material can also be treatled as a 
current generator. A $1 \mathrm{~mm}^{2}$ sample of piezoelectric material has generated $8 \mathrm{~A}$ of current, indicating that if the sizc were scaled to $7 \times 7 \mathrm{~cm}$, more than $5 \mathrm{kA}$ could be generated. When scaled to realistic sizes, these numbers show that piezoelectric material could produce electromagnetic pulses that could be radiated and used against a variety of targets.

Piezoelectric material is available in films 9 to $800 \mu \mathrm{m}$ thick with a thin 0.2 $\mu \mathrm{m}$ aluminum coating sputtered onto both sides for electrical contact. The film layers can be stacked so the voltage across one thickness adds to the voltage of the other thicknesses, such as when capacitors are connected in series. This piezoelectric material should be configured such that an explosive shock wave can compress the material, and with a geometry which allows connection to an antenna. We performed a POP experiment to become more familiar with the concepts that might lend to a useful device.

Although performed quickly, simply, and with unrefined diagnostics, the POP field tests demonstrated conclusively that electromagnetic radiation can be generated by piezoelectric material when a high-explosive driven shock wave is used to compress the material. The two configurations shown in Figs. 8.13 and 8.14 were tested at Firing Point 6 in Ancho Canyon in a collaborative experiment with AT-9 and M-6, the Shock Wave Physics Group; MEE-3, the Instrumentation Group; MEE-9, and the Engineering Design Group. The configuration in Fig. 8.13 illustrates that when we stack the layers of piezoelectric film, voltage is generated across the layers. A wideband bowtop antenna was used to radiate the energy. Physical dimensions of the film were $5 \times 5 \mathrm{~cm}$; 10 layers of $52-\mu \mathrm{m}$ thick film were stacked one on top of the other. This configuration used $300 \mathrm{~g}$ of Detasheet high explosive in two 4-mm-thick, $7 \times 7$ $\mathrm{cm}$ squares. The test configuration shown in Fig. 8.14 used a single 0.5mm-thick sheet of piezoelectric polymer. This configuration uses the material's capability to generate a large current to drive a loop antenna designed to resonate at $50 \mathrm{MHz}$.

Diagnostics employed at the firing point included five antennas covering overlapping frequency bands that ranged from a few megahertz to 12 $\mathrm{GHz}$. The antennas included a horizontal longwire doublet, a log periodic antenna (bandwidth $=20$ $\mathrm{MHz}$ to $1 \mathrm{GHz}$ ), two ridged waveguide horns with different bandwidths $(0.5$ to $6 \mathrm{GHz}$ and 1.0 to $12.4 \mathrm{GHz}$ ), and a Ddot probe (electrically small dipole). We observed radiation at frequencies ranging from a few megahertz to as high as 0.5 to $1 \mathrm{GHz}$ when the bowtop antenna with a 30 to $700 \mathrm{MHz}$ bandwidth was used to radiate the pulse. We observed rise times of approximately $3 \mathrm{~ns}$ and pulse widths as long as

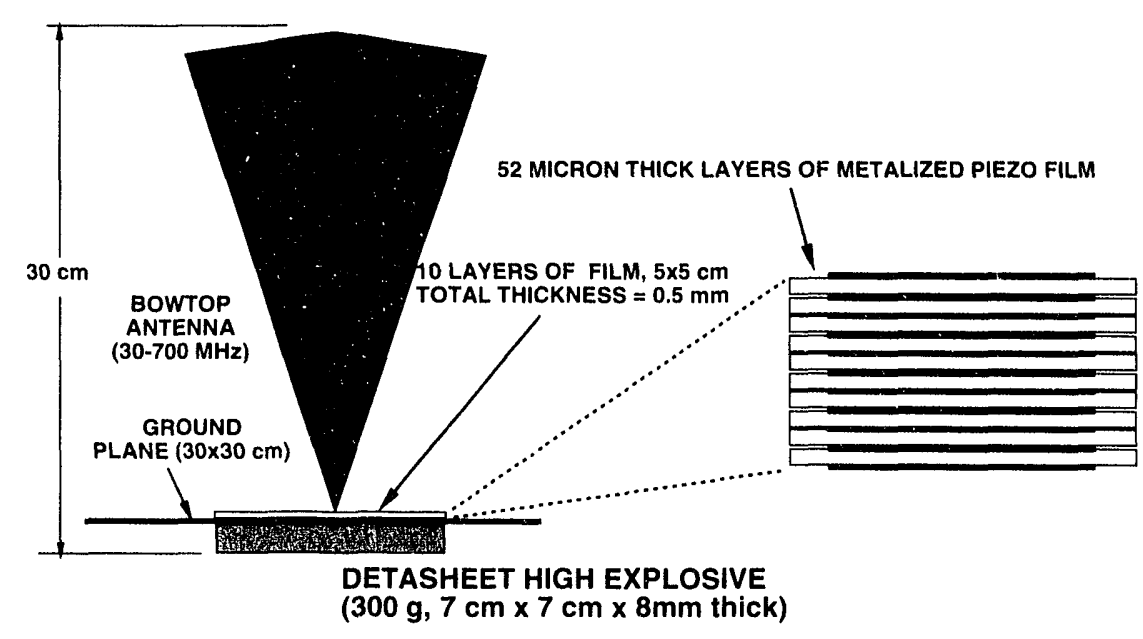

Fig. 8.13. Explosively driven if experimental configuration (Bowtop antemna). MvF 91-80

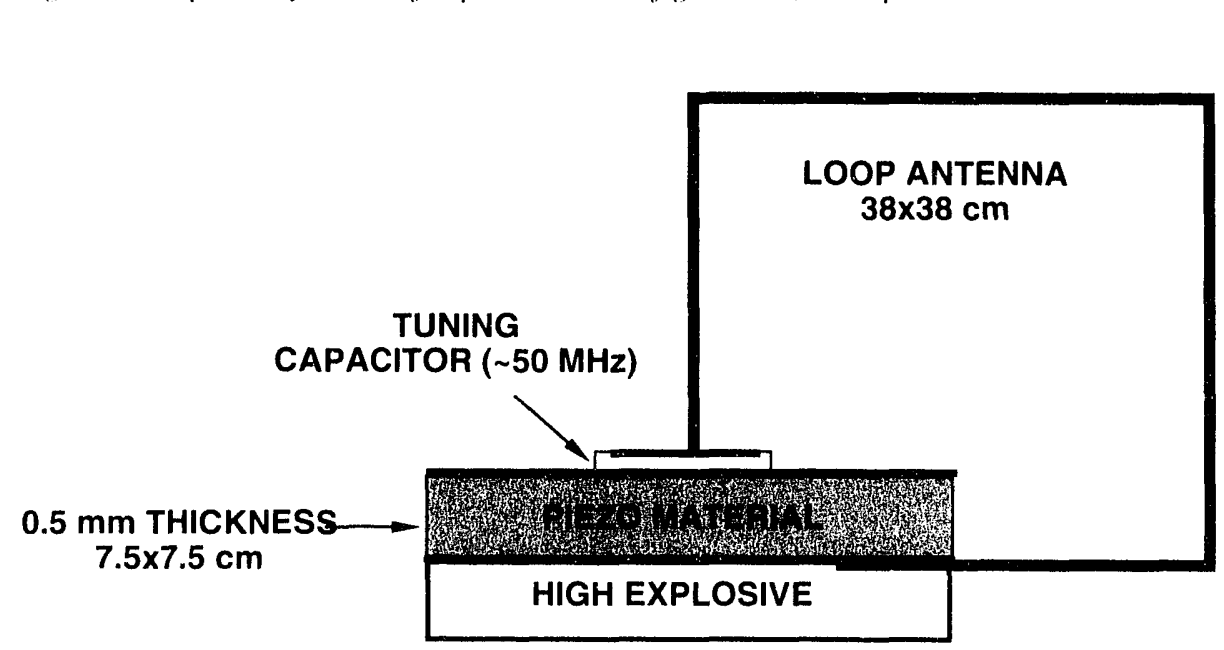

Fig. 8. 14. Explosively driven rf experimental layout (loop antenna).
$600 \mathrm{~ns}$ in the detected microwave signals. Figure 8.15 shows data from two different receiving antennas on the same explosive shot, when we used the Fig. 8.13 bowtop geometry. We estimated the radiated power densities at the antennas located 40 feet from the firing table to be tens of $\mu \mathrm{W} / \mathrm{cm}^{2}$.

Based on all five shots in two vastly different configurations (three with the bowtop and two with the loop antenna), these field tests demonstrated that electromagnetic radiation can be repeated generated by piezoelectric material that is compressed by a highexplosive driven shock wave This microwave source package is relatively compact and the quantity of high explosive is only a few hundred grams. 


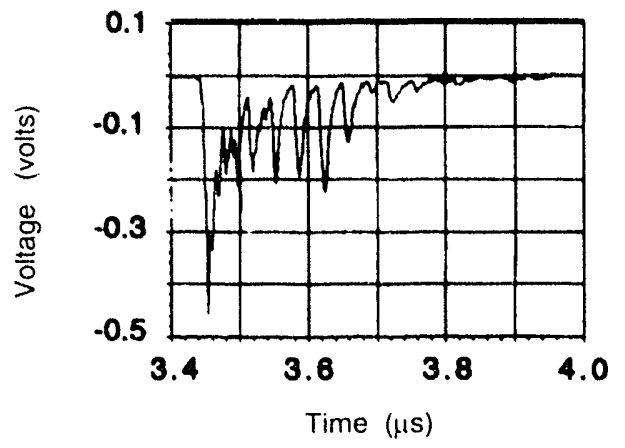

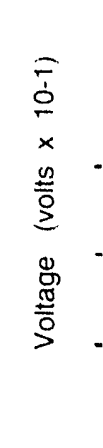

repetitively pulsed HPM sources and tor wideband, transicon pulse gencration. These applications reyuire outputs of tens of kilovolts to megavolts into loads of $5(0) 1010(0)$ ohms for natnosecond to microsecond pulse lengths at repetition rates up lo tens of kilohertz. Most of our developmental pulsers employ state-ofthe-art thyratron switches. These lubes are part of a long-term effort at Los Alamos to develop extremely highpowered repetitive switches for particle beams and lasers.

\section{BANSHEE} with personnel from five groups across four technical divisions, required an exeniplary level of skill, teamwork, and dedication by each member well beyond normal expectations.

\section{Pulsed-Power Research and Development}

concept. and implemented a proof of principle demonstration in less than 2 weeks The suceessful completion of
AT-9 is actively developing pulsedpower technology for advanced.
The BANSHEE pulser currently supplies a $6(0)-k V .10-k A, 2-\mu s$ pulse. It is being used as the electron-beam driver for the RKA experiment and ats a thyration test bed (Fig. 8.16). Recently, the CX1812 thyration tube produced by English Electric Valve (EEV) has been tested to failure. Currently, this thyratron tube, developed for the Strategic Defense Initiative Organization (SDIO)

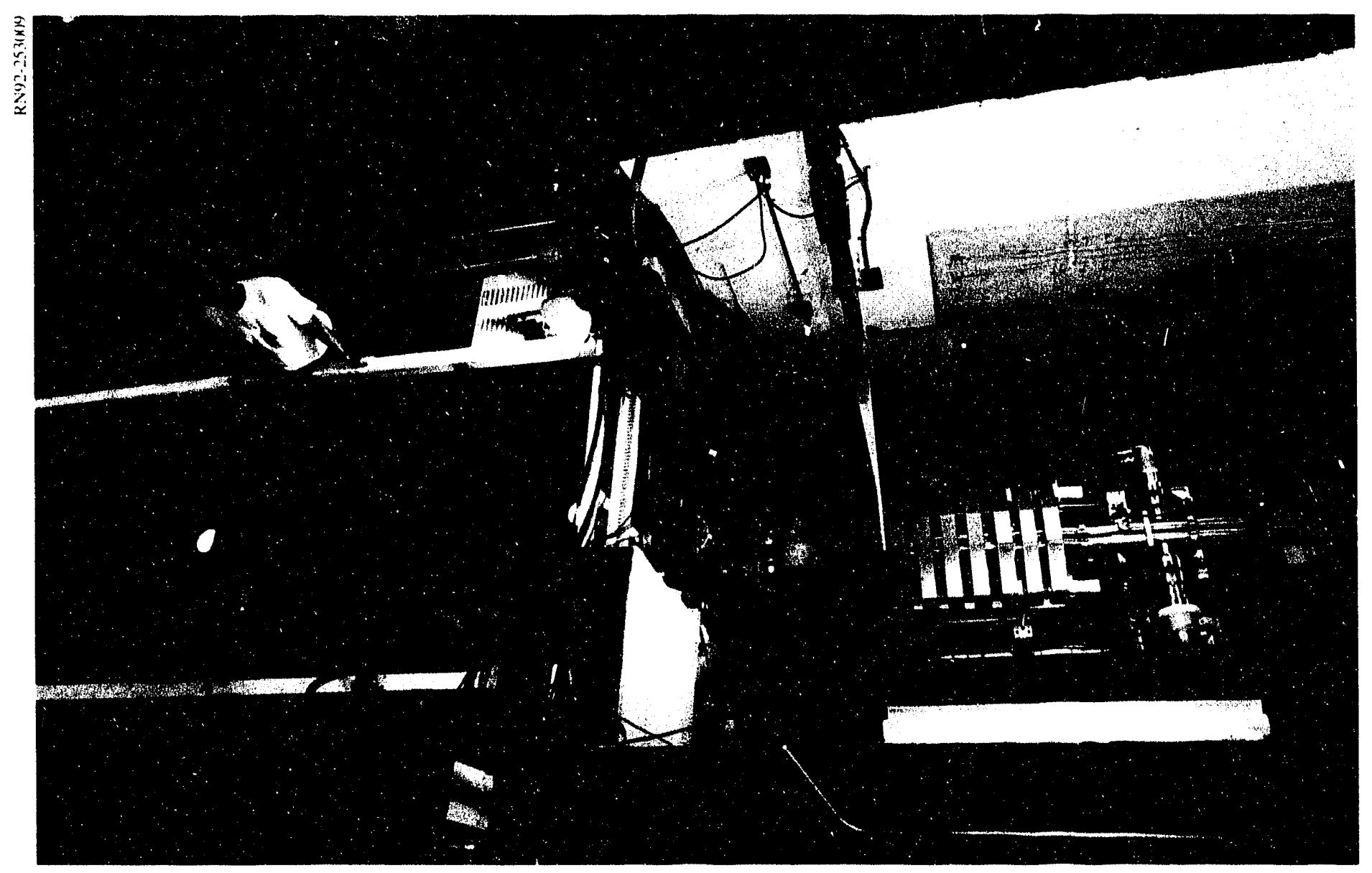

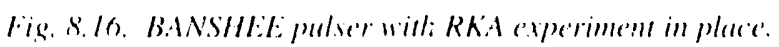


applications has the highest peak power of any thyratron produced. Several smaller tubos have been qualified at the muitigigawatt, microsecond level at up to $5 \mathrm{~Hz}$. EEV has corrected the flaws discovered in the first tests of the CX1812 and has produced an improved tube variant that will be tested in BANSHEE in 1993.

The tests to date have reached the limits of the pulsed-power system in several areas. An improved charging system and power supply have been designed and presented to sponsors. If funded, these would allow BANSHEE to operate in burst mode at the megawatt average power level. Improved capacitors have been identified that can operate reliably at this average power level.

\section{Portable Pulser}

The portable pulser is another advanced modulator developed for HPM source experiments. In 1992 this system was moved into the new AT-9 laboratory in building MPF-14, the first step in the system 's full-power commissioning. In 1993 we will add the shielding and diagnostic facilities, and the LOG experiments will be transferred to this machine, yielding a highly reprod scible 600-k V, 3-kA pulse. The system's ability to reproduce voltage will be a major improvement over the existing gyrotron power source, because the voltage repeatability between shots is critical when developing the complex beam optics in the cusp-injected electron gun.

\section{WEMPE 4}

A wideband electromagnetic pulse environment (WEMPE 4) was developed by AT-9 over the past several years for effects testing, developing antennas, and developing GPR. A turnkey WEMPE 4 system was constructed and delivered to the air force's Rome Air Development Center in 1992. Fig. 8.17. This is a userfriendly package supplied for wideband EMP experiments.

\section{Electron-Gun Test Stand}

Construction has started on an electron gun test stand, a 200-k V, 200-A, 15- Hz high-voltage modulator used for developing and testing electron-gun components. We will be able to test high-current density cathodes and parts of advanced electron guns independently of the microwave source experiments on BANSHEE.

\section{Vulnerability, Lethality, and Effects (VL\&E) Testing}

During the last 3 years, the Vulnerability, Lethality, and Effects (VL\&E)

Section in AT-9 has conducted many if effects tests, and the quantity of work is growing every year. This section is responsible for planning, executing, and documenting electromagneticcoupling tests for various government sponsors; data processing and analysis are an additional charter shared with Group X-5. The electromagnetic-

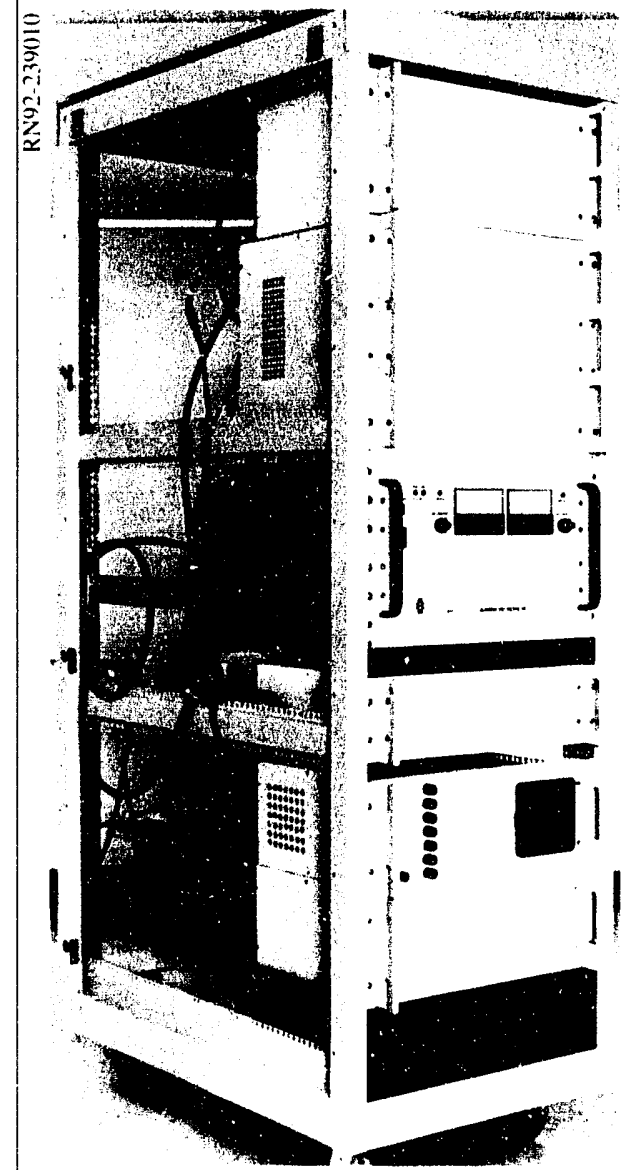

Fig. 8.17. Electromagnetic Pulse Generator delivered to Rome Air Development Center. coupling tests provide AT-9 with the opportunity to continue to develop ultrawideband video-pulse sources and to contribute critical coupling data to national committees such as the System Effects Assessment Team, the Foreign Asset Assessment Team, and the Department Of Energy (DOE) TriLab High Power Microwave (HPM) Vulnerability Committee. During FY 1992, the VL\&E Section conducted electromagnetic-coupling tests on three mobile military systems. These tests used a variety of electromagnetic environments to ensure complete electromagnetic characterization of each system.

\section{High-Level Testing of Mobile Military Systems}

During FY 1992, AT-9 conducted lowlevel frequency-domain tests on each system to build a baseline data set from which the response of the command post to high-ievel environments would be predicted. A typical system is shown in Fig. 8.18. The data acyuired during the high-level electromagnetic (EM) coupling test were then compared with the values predicted from the low-level data to evaluate the accuracy of numerical techniques used in generating the "pre-test prediction" values. After review of the data, we determined that the numerical techniques used to create the predicted values were valid, and we incorporated both the high-and low-level data into a transfer function data base. This data base is available to assist weapons designers in their evaluation of weapons's output in the context of the electromagnetic frequency coupling windows on that particular system. The test were conducted at the Defense Nuclear Agency's Advanced Research Electromagnetic Simulator (ARES): a facility originally built to test ICBM systems such as the MINUTEMAN and the PEACEKEEPER. However. recent modifications have given the facility the capability to conduct both low-level frequency domain and low-. medium-, and ingh-level time domain pulse testing. 


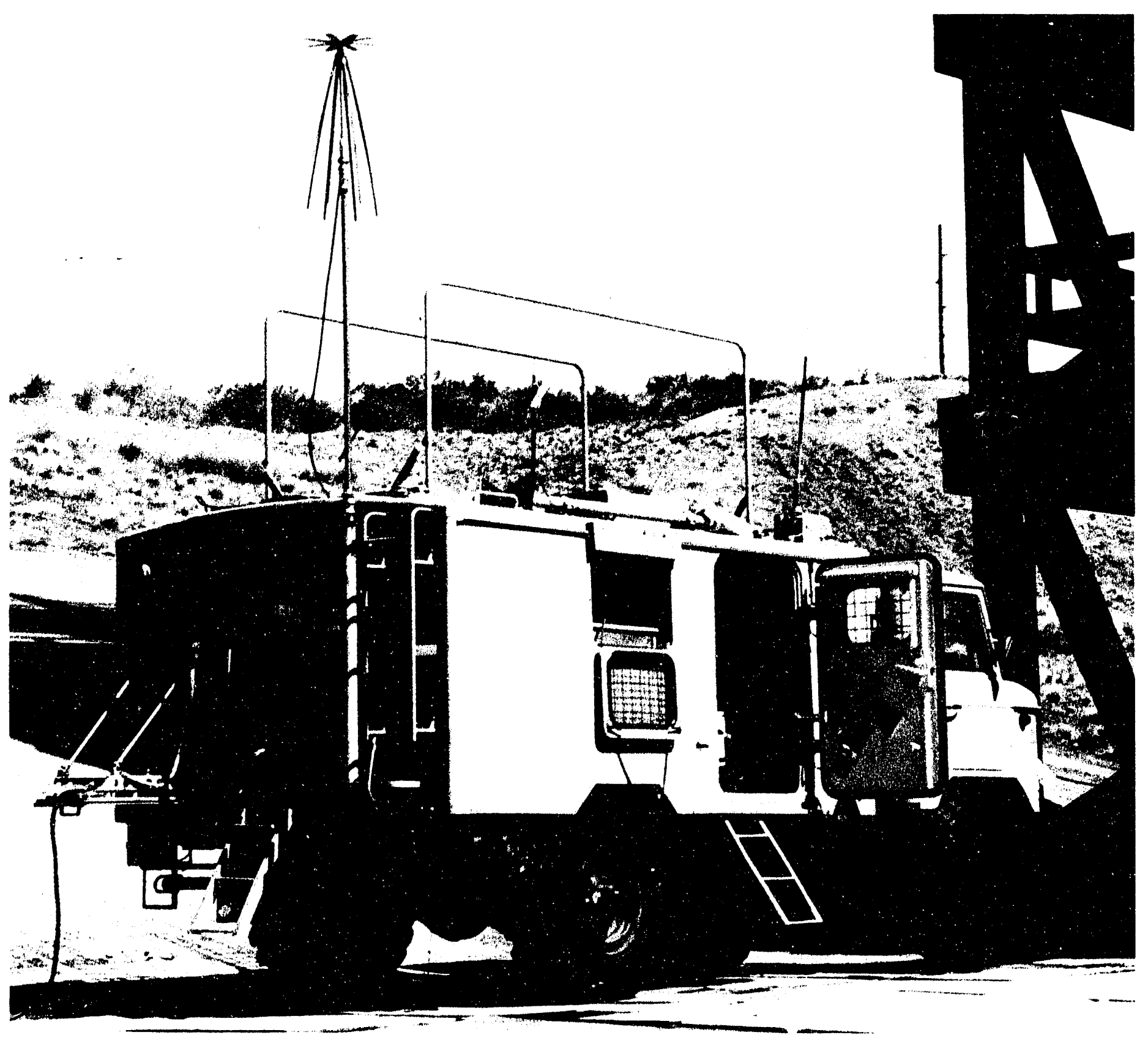

Fig. 8.18. Typical electronic system undergoing EMP VLEE te'sts.

\section{Test Point Impedance Assessment}

While the overall test methodology did not vary from that used in FY Iogl, we did use the kests lo complete development of a simple lechnique used 10 measure the impedance of at lest point in silll.

In the past, the HPM testing community has used an nominal value of 50 ohms ( $\Omega$, for the kest point impedance. This choice is heavily derived from $\mathrm{S}_{11}$ (rellected energy) meatsurements made on numerous test points and based on the fact that printed circuit designers use dimensions for the printed circuit that correspond to approximately $50 \Omega$ for frequencies $>$ (iltr. However, that value is mot valid hecaluse the circuit componems begin to dominate the circuit impedance al frepuencies $<1$ Gill. Wo believe the $S_{11}$ technicue is limiled to providing insight only into the trend of the data but not the actual value. During the $S_{11}$ measurement, the coupling path and lest point impedance are measured in parallel. However, when the system is illuminated in a free space $E M$ enviromment, the coupling path and lest point impedance are in series, resulting in al different voltage and corrent distribution as compared with the $S_{11}$ measurement. This led us (1) devise a simple method by which the test engineer could determine the test peint impedance in a realistic measurement sillation. 
Our new technique connects a shunt resistor across the test point to ground. The ground point is common to voltage probe and the test point circuit. Th ; creates a three-resistor parallel circuit that features two known (voltage probe and shunt resistor) and one unknown (test point impedance) resistances. Figure $8.19 \mathrm{a}$ shows the equivalent circuit at the test point. This parallel circuit is in series with the coupling aperture. With different values of the shunt resistor, we can obtain different transfer functions. Figure $8.19 \mathrm{~b}$ shows three such transfer functions that illustrate the change in response caused by the shunt register. By ratioing the "no shunt" case with the "1000 $\Omega$ " shunt and the " $50 \Omega$ " shunt, we can calculate the actual test point impedance at each frequency of interest. This technique is very useful because it is easy to implement and gives the test engineer an accurate measure of the effect of instrumentations on the test point response.

In FY 1993, four systems will be tested, with the first test scheduled to begin in March 1993. The systems will be tested at a variety of simulators because of the various requirements of our sponsors. The data acquired in FY 1992 will be used to improve the extrapolation techniques currently used in scaling low-level responses to highlevel environments and will become the backbone of system vulnerability analysis currently underway at Los Alamos. The data will also become an important cross-check for an ongoing EM modeling effort that is studying the coupling efficiency of an incident plane wave to a complex, lossy cavity. Additional smaller effects tests will be performed in conjunction with the GPR program to continue the development of untrawideband video pulsers and appropriate antennas used to propagate the new wave forms.

\section{High-Performance Ground Penetrating Radar (HIPERGPR)}

AT-9's goal is to produce an effective subsurface radar that has commercial value for ongoing environmental cleanup programs by developing the existing prototy pe at LANL into a fieldable system with substantially higher performance than existing systems. This prototype has four orders of magnitude more peak power and five orders of magnitude more average power than commercial GPR units.

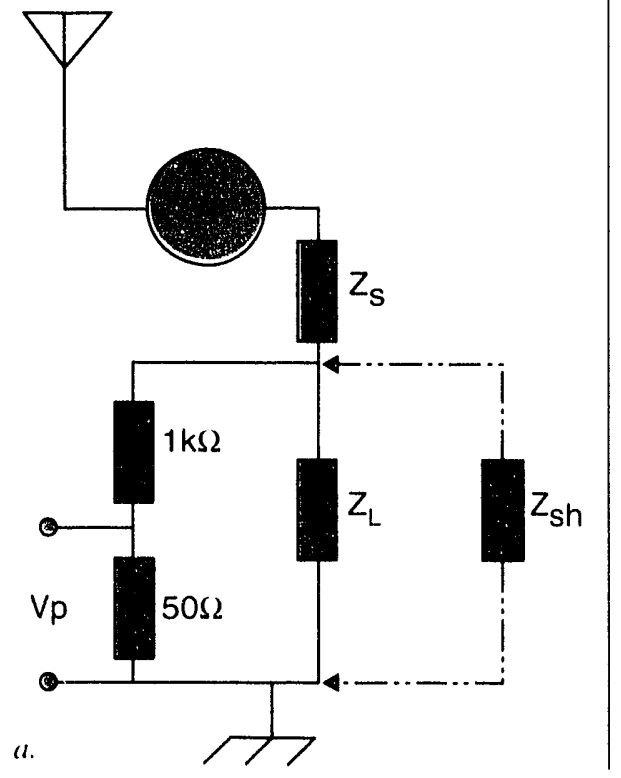

When combined with the proper radar receiver technology, these power increases result in the radar penetrating much deeper into the earth. The prototype high pertormance ground penetrating raddar (HIPERGPR) has demonstrated ien times greater depth range than a commercial GPR when tested at TA49. In FY 1992, our efforts with GPR focused on benchmarking the device's performance against realistic targets as well ats improving the system's signal processing and user interface.

The concept of cradle-to-grave control of weapons materials puts new requirements on defense programs (DP) to locitte buried waste and to continuously monitor disposal sites. Wideband high-power pulsers and diagnostics developed for the nuclealr effects program call be used to clean up DOE waste sites effectively, efficiently, and economically. Hence, the HIPERGPR with its grealter search range, caln perform noninvasive, subsurface surveys over realistically sized burial sites. Additionally, the HIPERGPR provides the unicpue additional capability to accurately measure the hurial depths.

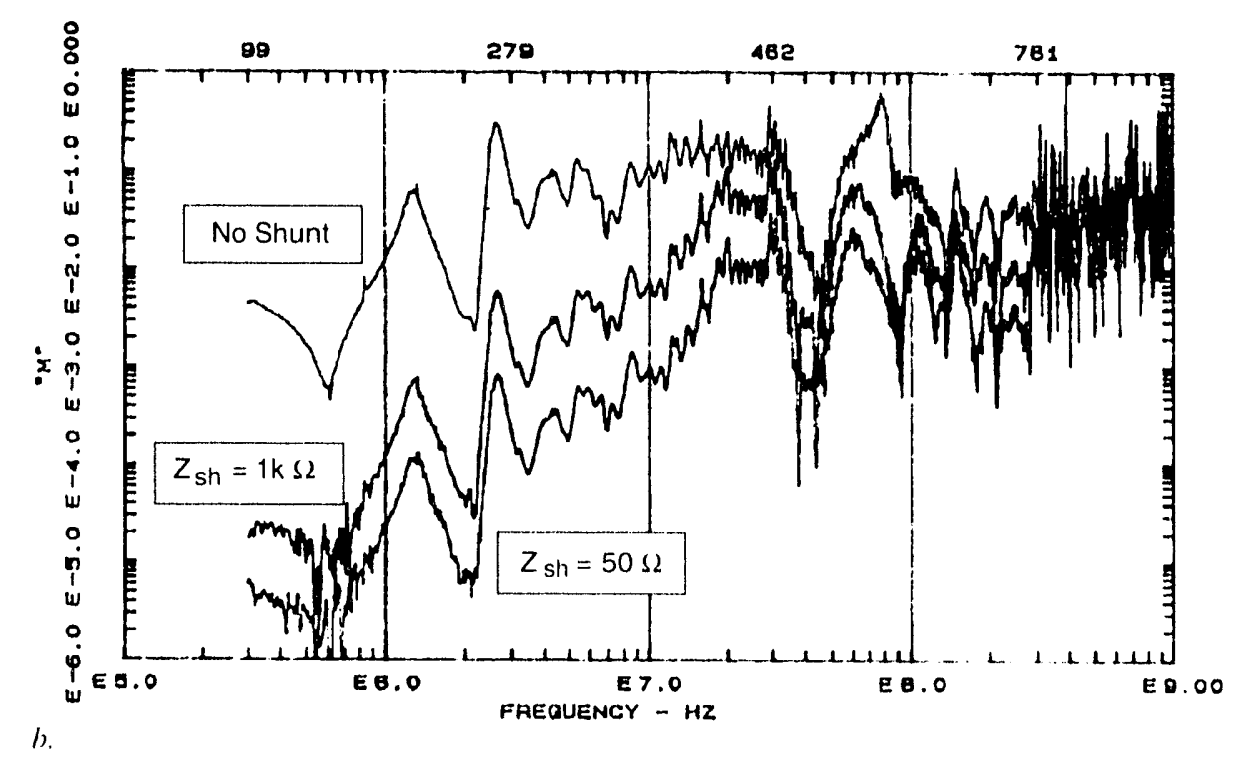

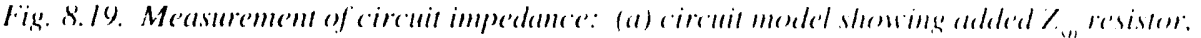

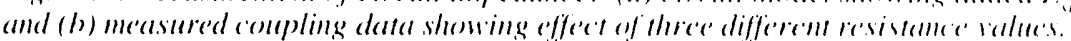




\section{Materials Processing with High-Power Microwaves (HPM)}

The application of high-power microwaves (HPM) to materials processing is a new project in AT-9 begun in 1992.

Initially, we are researching how microwave radiation interacts with coal in a collaboration with the Coal Technology Office at LANL. Further work involving microwave chemistry has also begun.

Coal, the most common fossil fuel, produces pollution as a byproduct of its combustion. This major limitation to its use has led us to investigate methods of cleaning up the coal fuel cycle.

Beneficiation is the process of removing pollutants before the coal is burned. Typically, less useful constituents such as sulfur and heavy metals are removed by a chemical cleansing process. A related technique is coal gasification or liquefaction in which the most valuable constituents are removed from the bulk of the coal leaving less useful and dirtier components as waste. AT-9 is investigating the interaction of microwave pulses with coal samples in an experimental effort to understand the flow of microwave energy into the coal on a short time scale. We believe that the coal will be heated and fractured allowing the volatile constituents to be released and separated.

Our experimental investigation of the interaction of microwave pulses and coal began with low power network. analyzer measurements on coal samples. The purpose is to learn the dielectric constant and loss tangent of coal as a function of frequency and to study the variations of these parameters among different samples. The next step involves setting up the chemical diagnostics for the high-power tests.

In collaboration with CLS-4 (Photo) Chemistry and Photo Physics), we evaluated various chemical analysis techniques from gas chromatography to mass spectroscopy, as well as more advanced techniques employed routinely in CLS. For the initial work, we focused on Fourier-transform infrared spectroscopy augmented by mass spectroscopy. Infrared spectroscopy is the preferred technique for analyzing the hydrocarbon gases expected to be evolved from the coal samples. An experimental sample collection system was set up in AT-9 as a precursor to the actual microwave processing, and several samples were analyzed at CLS- 6 . The next step is to irradiate coal samples using the 20$\mathrm{MW}, 1300-\mathrm{MHz}$ klystron in building MPF-14. A waveguide sample holder has been assembled for this purpose and is ready for use.

Microwave heating is becoming a useful tool of the industrial chemist. Many processes are aided by the selective and rapid heating produced by microwaves. AT-9 is looking at the possible benefits of high-power pulsed microwave energy for chemistry. Many chemical reactions are limited by competing reactions that interfere with the desired reaction. However, if microwave energy is applied in a pulsed mode, the desired interaction may be favored so that the overall system efficiency is enhanced. This has been demonstrated at low-power levels, and we are trying to employ the high-power sources and expertise in AT-9 to improve technique.

\section{Wideband Antenna Development}

The antenna development work in AT9 emphasizes broadband, high-peak power radiators for transient EMP used for wideband-VLE field testing and impulse radar. Our development goals are to maximize bandwidth and voltage breakdown capability while minimizing dispersion. The latest antenna developed is a modified ridged-waveguide transverse-electric mode structure used for GPR and supplied to Air Force and Army laboratories in 1992.

\section{0-MHz RF for the Advanced Free-Electron Laser}

The 20-MW. 1300-MHz klystron was brought back on line to support the AFEL experiment. Many improvements were made to the syitem's housekeeping in the wake of the Tiger Team visit. After th:se improvements were completed, the waveguide was reconnected to the compact linac of the AFEL and the system was promptly conditioned up to the 20)-MeV level (10 megawatts peak rf power). During 1992 klystron personnel worked closely with AT-7 personnel to commission the AFEL and diagnose and troubleshoot the beamline and subsystems. AT-9 worked closely with AT-7 and AT-5 to implement and test rf control systems on the system. The work with AT-5 was a high-power shakedown test of the University of Twente's low-level control system. This realistic test allowed for many small details to be corrected before the system was shipped to the Netherlands.

AT-9 proposed a design for a compact 1300)-MHz rf system intended to be a dedicated klystron stand for the AFEL. The design is a very compact package designed to minimize the AFEL size. It will employ a large klystron, the Litton L-3702, several of which are available in AT-9. This system will supply a longer pulse with higher peak power, as well as higher average power than the existing stand, and will be completely integrated into the AFEL computer automated control system. The construction of the 1300)-MHz rf system for the AFEL will be a collaborative effort between AT-5, AT-7, and AT-9 in 1993. 
Technical Highlights $-A T-9 \cdot$ Very High-Power Microwave Sources and Effects 

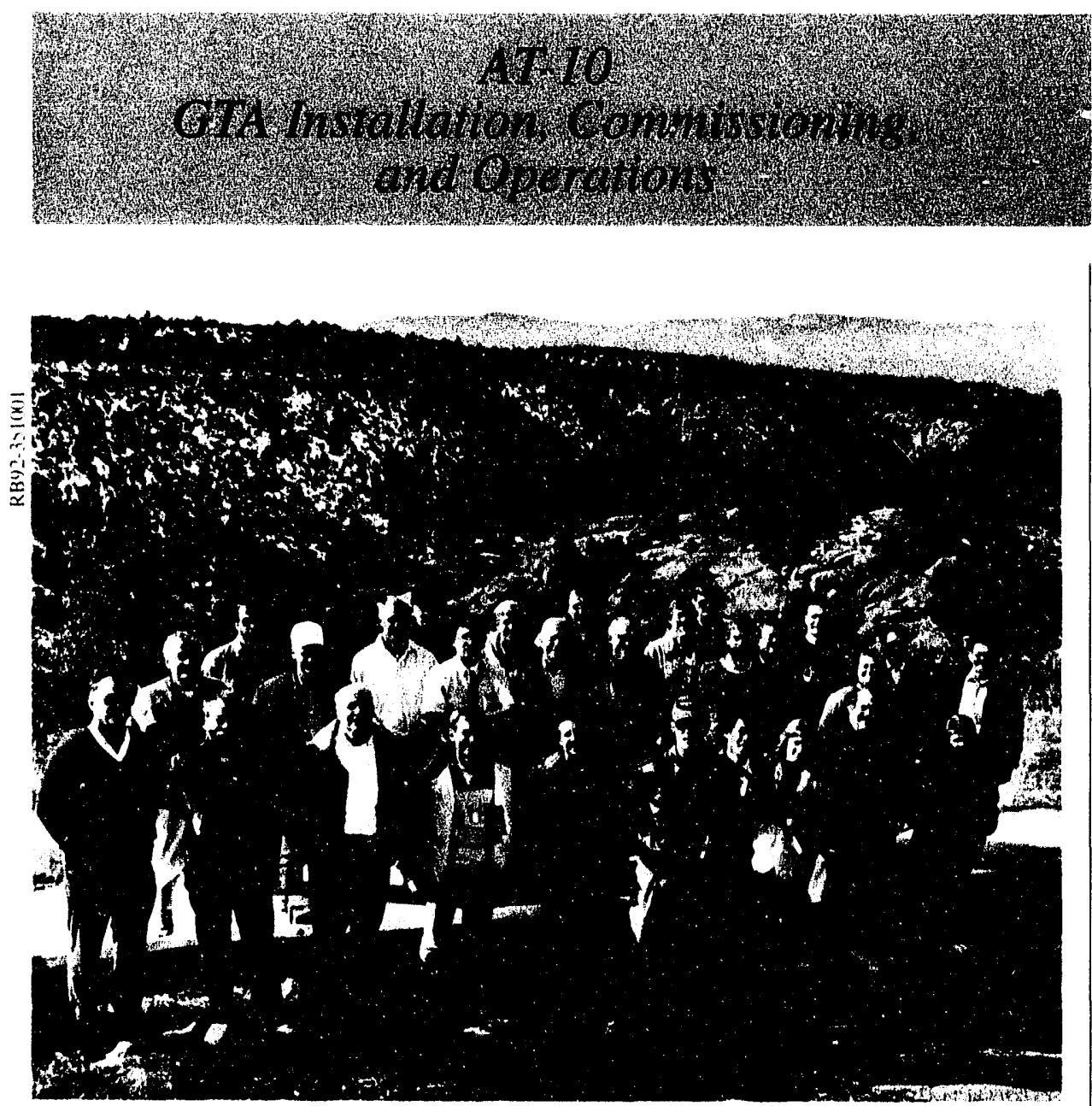

Introduction 109

Background 109

Achieve'ments 109

Injector Sistems. 109

GTA Beam Commissioming ... /1/ High-Power Cavity Conditioning and Preparation $1 / 1$ Safety Systems Modification . $1 / 2$ Cryogenic Cooling System ... / /2 Becamline Alignment $1 / 2$ Facility Support and Installation 113 DARHT Support 113

Future Plans 11.3 


\section{Introduction}

The primary mission of AT-10 has been to support Ground Test Accelerator (GTA) beam experiments. AT-10 is responsible for ion-injector development, final beamline installation of GTA components, and commissioning and operation of the accelerator. We perform the final radio-frequency (rf) cavity preparation, conditioning, alignment, and integrated checkout. Our responsibility includes supplying all facility interfaces, water and cryocooling, vacuum systems, and power wiring. Coordination of all beam testing or commissioning is done by AT-10, although specific experiments may be led by other groups.

Beam commissioning is the final step in the integrated quality-assurance process for verifying the performance of assembled beam hardware. We use extensive beam diagnostic equipment with integrated computer systems to measure beam parameters. Using these measurements, we compare actual performance with that predicted by beam-simulation codes. Validation of these codes is essential for improving confidence in future designs.

Group AT-10 was formed with its current mission in the fall of 1989; previously it had been AT-2, whose sole purpose since 1978 was to develop neutral particle beam (NPB) technology. Now the group's mission is changing again to reflect the broader responsibilities required by new projects. In the past, we concentrated on developing high-current, highbrightness negative-ion sources and injectors, such as those needed for the
NPB program. Although some negative-ion development will continue, we anticipate that most of our future work will require high-current, cw positive-ion or proton injectors. As a result, our latest thrusts are toward the integrated development of $\mathrm{cw}$ injector technology. AT-10 will continue to support GTA installation, operation, and commissioning. The experience gained and lessons learned in these endeavors will be applied to future ATDivision projects.

One of our senior staff members has been assisting with the design of and experiments on the Dual-axis Radiographic Hydrodynamic Test (DARHT) facility. In this instance, expertise developed on low-energy ion injectors is proving very useful for a highcurrent electron induction linac.

\section{Background}

Much of the group's work in FY 1992 was devoted to the commissioning and operation of the GTA hardware and characterization of the GTA beam. Cooling (at $20 \mathrm{~K}$ ) for the cryogenic GTA was provided by a 75()$-W$ helium refrigerator. Installation and acceptance tests of a 40-kW cryo-cooling system were completed this year. Future experiments will use the increased capacity of this boilinghydrogen cooling system.

We made a number of performance improvements to the GTA injector this year and proceeded with initial beam characterization of the intertank matching section (IMS) and the first GTA drift-tube linac (DTL) module. Meanwhile, we continued with the

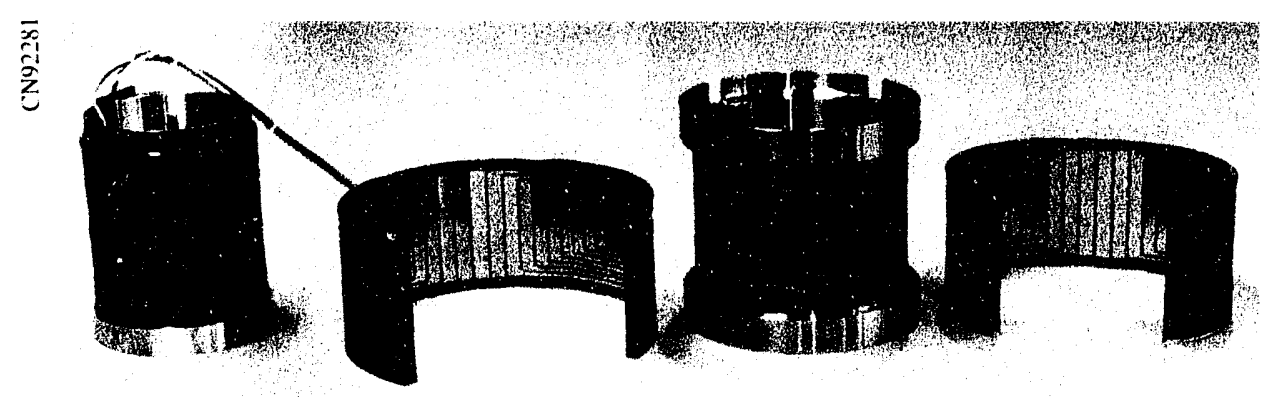

Fig. 9.1. Lambertson steering coils during assembly:

assembly of a cw $\mathrm{H}^{\text {ion source. This }}$ year marked our first serious entry into the design of high-current, proton ion injectors, a venture that may be essential for any of several emerging programs.

\section{Achievements}

\section{Injector Systems}

Operational reliability of the GTA injector was improved during FY 1992 , and consistent high-current, goodemittance beams were obtained. A modification of the extractor and emitter electrode designs permitted faster and easier electrode changes. Source reliability was improved by several upgrades to the control system hardware, the installation of improved Allen-Bradley modules, and the installation of noise-suppression electronics. Changes in start-up procedures, operating points, and tuning have contributed to make recent source operation more consistent and have produced brighter beams.

Two Lambertson steering coils were designed, built, and installed inside the bores of the two low-energy, beamtransport (LEBT) solenoid magnets. These coils give much-needed beam steering into the radio-frequency quadrupole (RFQ). Figure 9.1 shows these steering coils during assembly. A reduction in $\mathrm{LEBT}$ length earlier in the year was instrumental in reducing emiltance growth between the ion source and RFQ.

On the off-line discharge test stand (DTS), we installed a new $4 \mathrm{X}$ ion source that incorporates several design upgrades. The most significant upgrade is a new gas-pulser that uses a modilied, low-cost, automotive fuel-injection valve to replace the previous piezoelectric valve. The new system reduced the total gas flow by nearly a factor of 3 , without affecting arc operation. A reduction in hydrogen consumption may be important for reducing enittance growth in the LEBT. Conliguration and cooling improvements were also made on this new source. 
We hatve designed. huilt, and tested a new controllable arc-currem pulser on the DTS. This pulser uses HEXIJT/s lo control the source are current; the HEXFETS replace the transistor on/off switch and ballast essistors. With this are-current comtrol. we have the possibility of programming the time dependence of the ate current during the are matropulse.

Using internal Laboratory funding. we developed initial designs of a highcurremt. cow protom injector, suitable for projects such as the accelerator transmutation of waste (ATW) or the accelerator production of tritium (APT). A very similar D' injector might be needed for a Fusion Materials Iradiation falcility (FMIF). To laciliante our design effort. we compiled anailable information from cw lest stands both past and present. An extraktor and solenoidal-focusing LEBT were designed for 75-keV, 140mA hydrogen-ion beams. The integrated design wats presented at internal
AP'T and Acederator Based Conversion reviews.

We have also used internal funding (o) continue collaboration with the Chalk

River Lahoralory (CRL,) and (irumman Corp. In FY 1993, we will ship \$5M of operating cw test-stand equipment from CRL io Los Alamos. Continued testing and development of this cw equipment at Los Alamos in subsequent years should strengthen our design capability for high-currem proton accelerators.

In related work, we analyzed dala recorded in previous years with a fourgrid electrostatic analyzer to determine details of the space-charge neutralization of high-current $\mathrm{H}$ beams in lowenergy transport. This diagnostic determines the internal beam potential by measuring the energy cutoff of the escaping charged particles. Using the measured energy distribution of the ejected positive ions. we measured the degree of neutralization or compensation as a function of argon and xenom

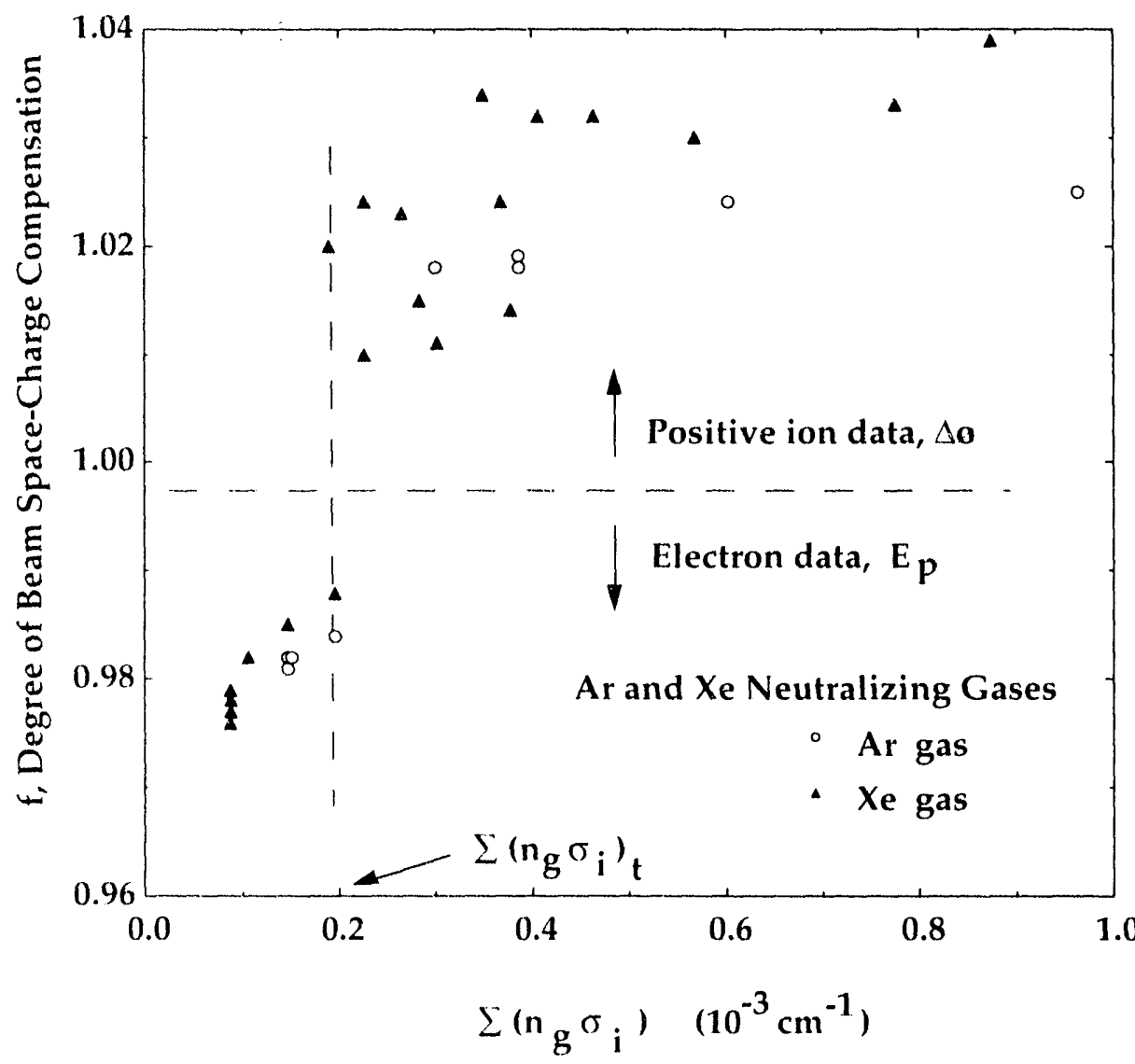

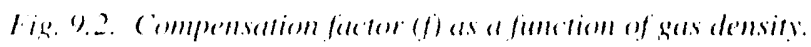


cooling water system on the modified HCTS. Testing of this $\mathrm{cw} \mathrm{H}^{-}$ion source should commence early in FY 1993.

Using the pulsed $8 \mathrm{X}$ source, we found that placing a conical collar at the emission aperture reduced the $\mathrm{e}^{-} / \mathrm{H}^{-}$ratio to $0.9 / 1$ w th no decrease in the $\mathrm{H}^{-}$beam current. Without a collar, the typical $\mathrm{e}^{-1}$ $\mathrm{H}^{-}$ratio is between $4 / 1$ and $5 / 1$. A barium cathode was tried in the $4 \mathrm{X}$ source in place of the cesium-coated, molybdenum cathode after we learned that a barium insert near the emitter of a cusped-field volume source increases the $\mathrm{H}^{-}$current from that source by about a factor of 3. All indications are that the $4 \mathrm{X}$ source $\mathrm{H}^{-}$output will be very low and the $\mathrm{c}^{-} / \mathrm{H}^{-}$ratio will be very high if a barium cathode is substituted for a cesium-coated, molybdenum cathode; therefore, we plan to use only cesiumcoated, molybdenum cathodes in the $8 \mathrm{X}$ source.

\section{GTA Beam Commissioning}

A series of GTA beam runs were completed during FY 1992. In the spring, we fully characterized the output beam and operation of the IMS. This structure includes two of bunching cavities and six permanent-magnet quadrupoles, two of which can be translated for beam steering and four of which have adjustable field strengths. The purpose of the IMS is to longitudinally and transversely match the RFQ output beam to the following DTL.

By maximizing RFQ transmission, we determined the optimal RFQ input match. The resulting Courant-Snyder parameters agreed with predictions.

During IMS testing, we measured both the transverse and longitudinal beam emittances for a variety of IMS quadrupole and buncher field strengths. We also measured the effects of beam steering in the IMS. The LINDA diagnostic remains the predominant method for characterizing the longitudinal-beam properties. In spite of identified difficulties, microstrip probes were used extensively for beam-centroid position monitoring at several locations. Beam energy and phase were measured versus IMS buncher field, giving excellent agreement with design predictions. Figure 9.3 shows the observed buncher gap voltage versus cavity power and shows that the design gap voltage occurs at predicted power level. The beam steering and emittance measurements are being used to produce a steering and matching model of the IMS. These models are being used again in DTL experiments to verify the predicted performance of the DTL.

We conducted the first beam operation of DTL module 1 in September 1992 and produced a $3.2-\mathrm{MeV}$ output. Future runs are planned for November 1992. We measured the transverse emittance of the DTL beam for a variety of input beam conditions and DTL operating set points.

\section{High-Power Cavity Conditioning and Preparation}

We have successfully conditioned three DTL modules. A successful conditioning consists of operating the modules at $20 \%$ over the required power levels for 2 -ms rf pulse durations. The modules must also operate for long periods of time (tens of minutes) with little or no breakdown. As in the case of the RFQ, the DTL module 1 reconditions in less than 0.5 hour after extended exposure to the atmosphere and immediately each morning after an 8-hour nonoperation period.

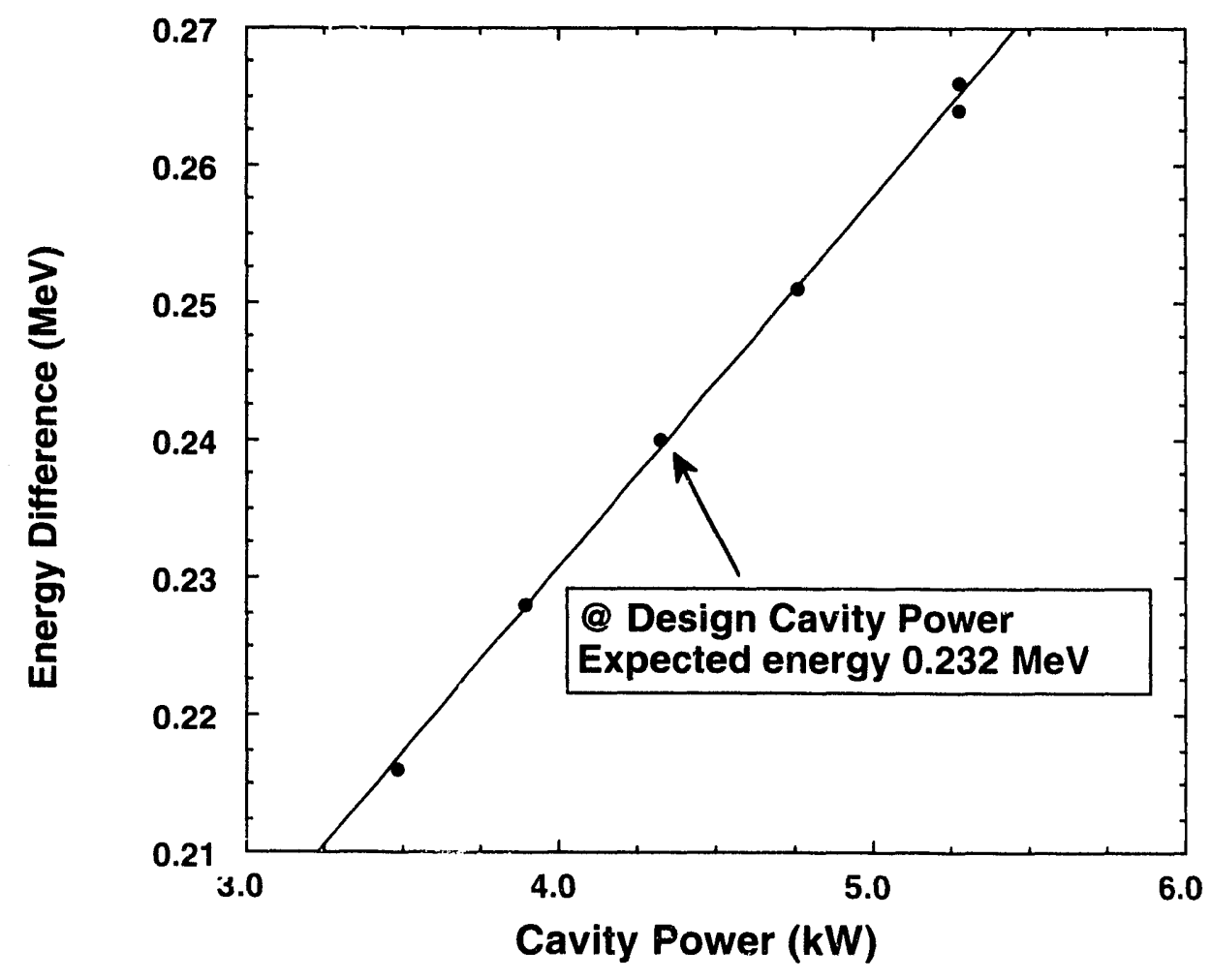

Fig. 9.3. Energy difference between acceleration and deacceleration modes for anity $A$. 


\section{Safety Systems Modification}

The Radiation Protection Safety System was modified to include the first DTL module. This hardwarebased system is modeled on the one used at Clinton P. Anderson Meson Physics Facility (LAMPF) and is designed to exclude personnel from the beam area whenever a radiation hazard might exist from beam operations. All GTA beam operations (with the exception of the 750-W cryo refrigerator) are conducted from the fully functional control room.

\section{Cryogenic Cooling System}

We presently cool the GTA beamline components to cryogenic temperatures with a Koch $750-\mathrm{W}$ refrigerator. We operate a second refrigerator during multiple-magnet taut-wire (MMTW) measurements made on the IMS and DTL modules at cryogenic temperatures. The new $40-\mathrm{kW}$ cryogenic cooling systern (CCS) will be required for all GTA experiments that involve more than the RFQ, IMS, and first DTL module. This CCS obtains its prelimi- nary cooling through a $L N$, to gaseous helium (GHe) heat exchanger and its primary refrigeration capability from a helium-to-liquid-hydrogen heat exchanger. The coolant pressure is 324 psig and the coolant flow rate is $484 \mathrm{~g} / \mathrm{s}$ at $60 \%$ of the turbo-compressor speed.

We completed the final construction of the CCS and numerous reports and studies for the operational readiness requirements. On May I, we received approval to take delivery of 15,000 gallons of liquid hydrogen to support the system acceptance tests which were successfully completed on May 8.

Figure 9.4 shows the compressor and mechanical components of the CCS.

\section{Beamline Alignment}

During FY 1992, we internally aligned the drift tubes (DT) in four DTL modules to an accuracy of 0.001 in. at room temperature. The goal is to maintain 0.002 in. alignment when operational at $20 \mathrm{~K}$ and under vacuum. A second MMTW alignment system was built and installed in an off-line cryo-vacuum test vessel. Measure- ments in this vessel confirmed that the required alignment was maintained under vacuum and cryogenic conditions for the first four DTL modules. We have adapted the MMTW system to measure not only the DT magnet centers, but also the magnet roll angle. We found that all DT magnet roll angles met the \pm 0.5 degrees specification except one that had a one-degree roll angle.

We completed a number of floor layouts in preparation of moving the existing accelerator and setting the ten DTL modules. We also performed alignment for the Advanced Free Electron Laser.

While on an off-site assignment to AccSys Technology, one of our engineers provided linac design support for the Superconducting Super Collider Laboratory. He provided the analysis of the DTL temperature control system and the specification of the cooling and heating systems. He also designed and specified the rf power waveguide and window assembly.

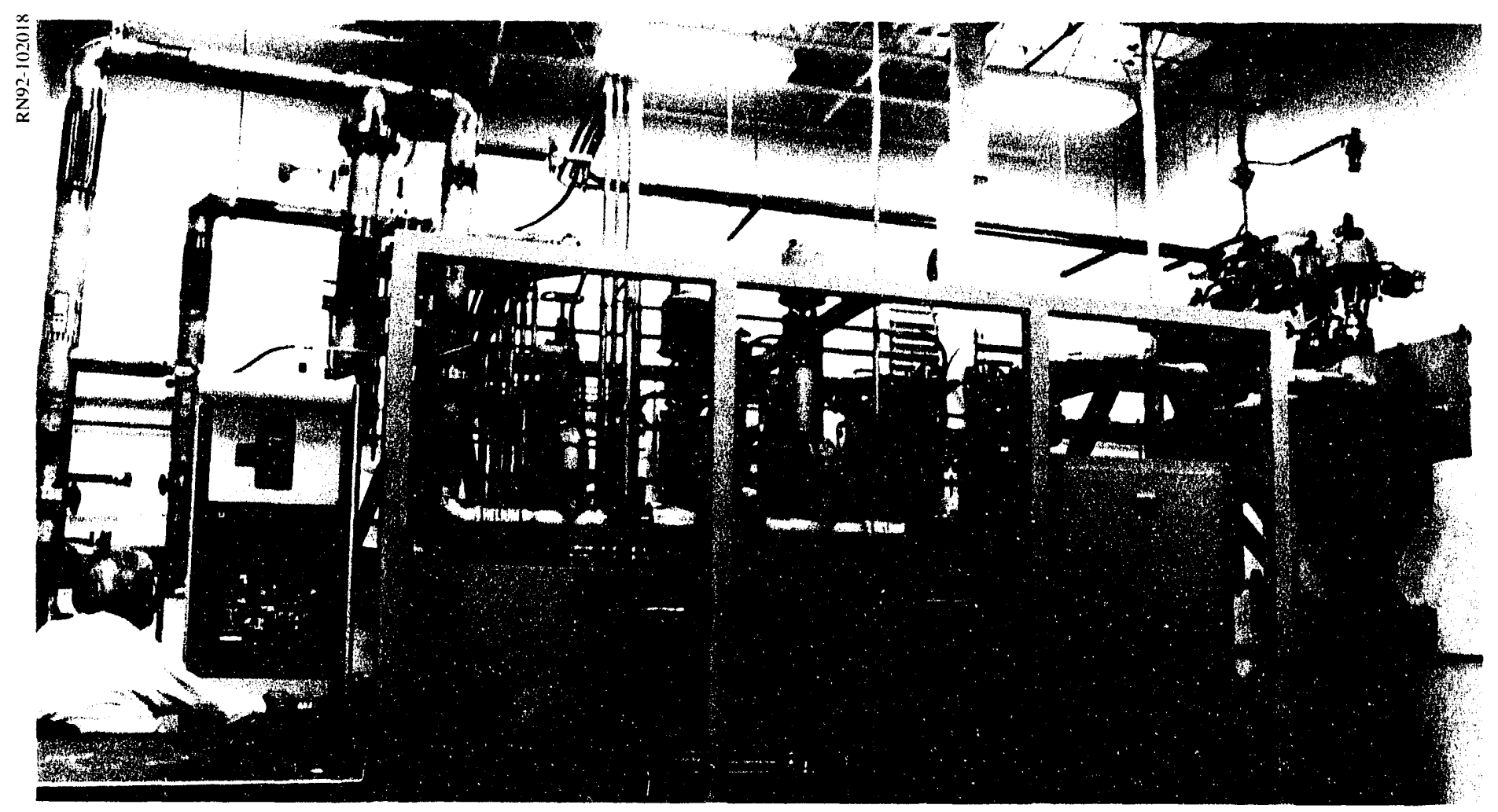

Fig. 9.4. Compressor and mechanical components of the CCS. 


\section{Facility Support and Installation}

AT-10 installed all GTA vacuum vessels and fulfilled all operational vacuum and cooling requirements. We continue to operate the helium refrigerators for GTA beamline experiments, high-power conditioning, and alignment verifications at cryogenic temperatures. All upgrades, additions, and maintenance to the GTA facility building as well as GTA electrical installations are our responsibility. Electrical installations include the of mezzanine utility rack and distribution systems: CCS Instrumentation and Control, fire alarm, and protection systems; the telephone and paging system upgrades; experiment $2 \mathrm{~A}$. beamline power-distribution system; control room and computer rooms electrical additions; power distribution to the mock-up DTL vacuum tank area; environmental, safety, and health upgrades and electrical power distribution system remodels; ground planes installations in the rf power area; and experiment 2D and 3 injector power trunk wiring installation.

\section{DARHT Support}

One of our senior staff members has been actively supporting the DARHT induction-linac electron accelerator project for the past 3 years. The first stage of the DARHT accelerator was completed this past year, and measurements of the 6-MeV, 3-kA beam are under way. Modifications of the injector pulsed-power system reduced voltage fluctuations during the pulse flattop to less that $1 \%$ rms.

Insulator failures on the 4-MeV DARHT injector appeared to result from insulator surface charging. A device was built that measures and corrects if necessary the pulse-to-pulse charge buildup. In initial tests, this device discharged the insulator rings after each pulse; the tests for solving the insulator-breakdown problem were very encouraging.
AT-10's major responsibility has been to debug and use beam dynamics codes for the end-to-end electron-beam simulation. With this code, linear beam dynamics and nonlinear beam-breakup centroid motion can be tracked as a function of time during the beam macropulse. Scme results indicate that space-charge effects are critical to the beam-breakup motion and can be mitigated only by careful design of the beam-transport system. AT-10 has also contributed to the data acquisition and analysis systems on the magnetic spectrometer, which will measure the time-dependent beam energy waveform to less than $0.5 \%$.

\section{Future Plans}

For much of FY 1993, we will be preparing test-stand hardware for testing components and systems needed for high-current $\mathrm{H}^{+}$and $\mathrm{H}^{-} \mathrm{cW}$ ion injectors. The initial test-stand configuration will permit us to test and compare several candidate ion sources. Later expansions of these stands will allow us to add and test transport components and, finally, to match beams into a RFQ.

Possible future projects such as the APT, the ATW, the FMIF, and possible major expansion of LAMPF and Manuel Lujan, Jr. Neutron Scattering Center will all require about $100 \mathrm{~mA}$ of protons or $\mathrm{H}^{-}$ion currents. Individual components have been developed that might meet these basic needs, but our primary focus will be to integrate the technologies needed for building a practical ion injector.

We will continue to install GTA components and will move the GTA to a new location within the beam tunnel in preparation for experiments $2 \mathrm{D}$ and 3 . In support of this move, we will supply all the utilities and will complete the floor installation. We will again lead the commissioning efforts on the first GTA DTL module and on the modified IMS and will work with the other GTA groups in analyzing the data. We plan to complete the alignment and the high-power conditioning of the remaining DTL modules. 


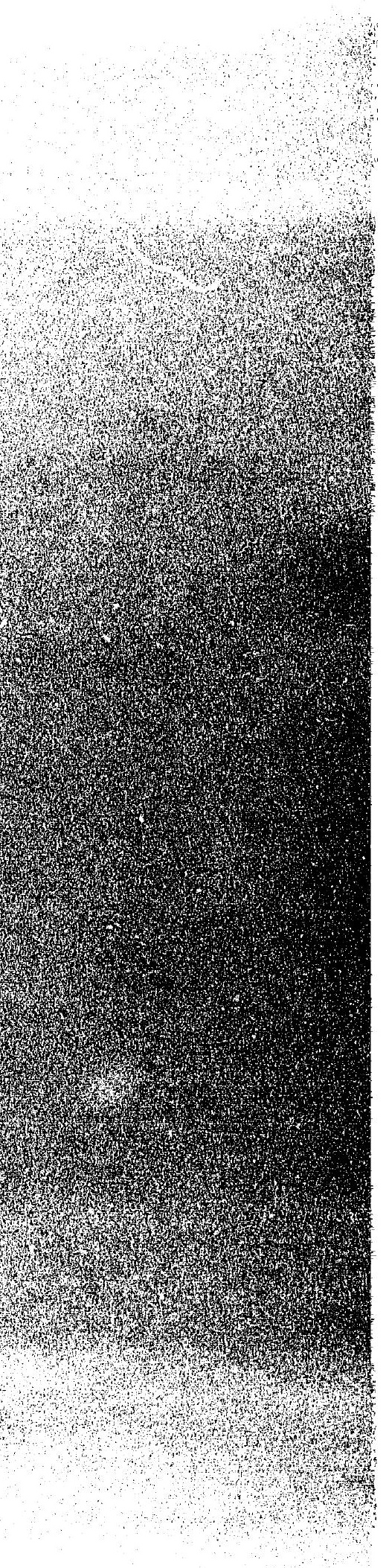




\section{AT-DO}

R. A. Jameson, G. P. Lawrence, and S. O. Schriber, "Accelerator-Driven Transmutation Technology for Energy Production and Nuclear Waste Treatment," European Particle Acceleratory Conference, Berlin. Germany, March 1992, Los Alamos National Laboratory document LA-UR-92-865.

R. A. Jameson, "On Scaling and Optimization of High-Intensity," to be published in the Proceedings of the Third Workshop on Advanced Accelerator Concepts, Long Island, NY, June 14-20, 1992, Los Alamos National Laboratory document LA-UR92-2474.

R. L. Sheffield, "Extreme Ultraviolet Free Electron Lasers," 1992 IEEE Lasers and Electro-Optical Society Annual Meeting, Boston, MA, November 1992, Los Alamos National Laboratory document LA-UR-92-2629.

R. L. Sheffield, B. E. Carlsten, and L. M. Young, "High-Brightness Linac for the Advanced Free-Electron Laser Initiative at Los Alamos," 1992 Linac Conference, Ottawa, Ontario, Canada, August 23-28, 1992, Los Alamos National Laboratory document LA-UR-92-2804.

R. A. Hardekopf, "Workshop on Plasma Focus Device Utility for Intense Neutron Source Applications," Los Alamos, NM, July 21-22, 1992, Los Alamos National Laboratory document LA-UR-92-3552.

E. A. Heighway, "The Path to the Neutral Particle Beam (NPB) Weapon," 1992 NPB Symposium, Argonne, IL, April 1992, Los Alamos National Laboratory document LA-CP-92-241.

\section{AT-1}

G. J. Vogt, F. D. Gac, J. D. Katz, B. Rusnak, and W. P. Unruh, "Microwave-Driven Spray Drying Annual Report, FY 1991," Los Alamos National Laboratory document L.A-UR-92-660.

G. Spalek, "Developmental Cavity Dimensions," Los Alamos National Laboratory document LA-UR-92-1246.

G. Spalek, "Voltages and Multipacting Regions in Coaxial Line Choke Joints," Los Alamos National Laboratory document LA-UR-92-1344.

G. Spalek, "805 MHz Single Cell Test Cavity End Flange Power Dissipation and HPP Coupler External Q's," Los Alamos National Laboratory document LA-UR92-1.345.

G. Spalek, "Maximum Electric Field in 50 OHM Coaxial Lines for rf Couplers," Los Alamos National Laboratory document LA-UR-92-1346.

G. Spalek, "Single Cell $805 \mathrm{MHz}$ Test Cavity Field Calibration Constants Shape Same as End 1/2 Cells of 7 Cell Developmental Cavity," Los Alamos National Laboratory document LA-UR-92-1347.

G. Spalek, "Variable Coupler Chock Joint Bandwidth," Los Alamos National Laboratory document LA-UR-92-1348. 
G. Spalek, "Developmental 7-Cell Cavity 2" Coax of Power Coupler Travel," Los Alamos National Laboratory document LA-UR-92-1349.

G. Spalek, "Single Cell $805 \mathrm{MHz}$ Test Cavity Field Calibration Constants," Los Alamos National Laboratory document LA-UR-92-1350.

G. Spalek, "Summary of Tuning Stresses and Loads for the 7-Cell Developmental Superconducting Cavity," Los Alamos National Laboratory document LA-UR-921351.

G. Spalek, "Bandwidth of Quarter Wavelength Stub Supported Coaxial Line," Los Alamos National Laboratory document LA-UR-92-1814.

E. R. Gray, "Helium Displacement," Los Alamos National Laboratory document LA-UR-92-2330.

R. W. Garnett, D. J. Liska, G. P. Lawrence, and T. H. Larkin, "Design and Simulation of a Bridge-Coupled DTL Structure for the $20-80 \mathrm{MeV}$ Region of a Proton Linac for Accelerator Transmutation of Waste (ATW)," 1992 Linac Conference, Ottawa, Ontario, Canada, August 23-28, 1992, Los Alamos National Laboratory document LA-UR-92-2663.

B. Rusnak, J. N. DiMarco, W. Diete, R. G. Maggs, A. H. Shapiro, and P. V. Wright, "Evaluation of Surface Contamination and Cleaning Techniques on Superconducting rf Cavities," 1992 Linac Conference, Ottawa, Ontario, Canada, August 23-28, 1992, Los Alamos National Laboratory document LA-UR-92-2754.

L. M. Young and S. Nath, "Effect of Transients on the Beam in the SSC Coupled Cavity Linac," 1992 Linac Conference, Ottawa, Ontario, Canada, August 23-28, 1992, Los Alamos National Laboratory document LA-UR-92-2768.

D. Schrage, L. Young, J. Billen, W. Clark, R. DePaula, G. Neuschaefer, P. Roybal, J. Stovall, and A. Naranjo, "Radio Frequency Quadrupole Lihnac for the Superconducting Super Collider," Application of Accelerators in Research and Industry Conference, Denton. TX, November 2-5, 1992, Los Alamos Nation.1 Laboratory document LA-UR-92-2962.

D. Schrage, L. Young, R. Aiken, W. Clark, R. DePaula, C. Gladwell, F. Martinez, A. Naranjo, P. Roybal, and J. Stovall, "University of Twente Photocathode Linac," Application of Accelerators in Research and Industry Conference, Denton, TX, November 2-5, 1992, Los Alamos National Laboratory document LA-UR-92-2963.

T. P. Wangler, A. G. Cimabue, J. L. Merson, R. S. Mills, R. L. Wood, and L. M. Young. "Superconducting RFQ Development at Los A.lamos," 1992 Linac Conference, Ottawa, Ontario, Canada, August 23-28, 1992, Los Alamos National Laboratory document LA-UR-92-2968.

J. E. Stovall, "A Review of RF Photocathode e- Sources," 1992 Linac Conference, Ottawa, Ontario, Canada, August 23-28, 1992, Los Alamos National Laboratory document LA-UR-92-3034.

T. P. Wangler, A. G. Cimabue, J. L. Merson, R. S. Mills, R. L. Wood, and L. M. Young, "Superconducting RFQ Development at Los Alamos," 12th Intl. Conf. on the Application of Accelerators in Research and Industry. Denton, TX, November 2-5, 1992, Los Alamos National Laboratory document LA-UR-92-3076. 
R. L. Wood, L. M. Young. D. J. Aikin, W. L. Clark, R. F. DePaula, C. Gladwell, J. E. Ledford. F. A. Martinez, and J. E. Stovall, "Photocathode Electron Linac for AFEL." Application of Accelerators in kesearch and Industry Conference, Denton, TX, November 2-5, 1992, Los Alamos National Laboratory document LA-UR-923215 .

\section{AT-3}

J. D. Gilpatrick. "RFQ and IMS Commissioning Beam Diagnostics Measurements." Los Alamos National Laboratory document LA-UR-91-3965.

B. Elind. "A Choice of Point-to-Parallel Focusing Modules," Los Alamos National Laboratory document LA-UR-92-422.

R. H. Kraus. Jr., "Permanent-Magnet Material Applications in Particle Accelerators," Twelfth Int. Workshop on Rare Earth Magnets and their Applications, Canberra. Australia, July 12-15, 1992, Los Alamos National Laboratory document LA-UR-92-1677.

R. E. Shafer and J. Johnson, "Electrical Characteristics of the SSC MEB Magnet String," Los Alamos National Laboratory document LA-UR-92-1766.

R. E. Shafer and K. Smedley, "Measurement of AC Electrical Characteristics of SSC Superconducting Dipole Magnets," XVth International Conference on High Energy Accelerators, Hamburg. Germany, July 20-24, 1992, Los Alamos National Laboratory document LA-UR-92-2179.

R. E. Shafer and Kay Smedley, "Electrical Characteristics of Long Strings of SSC Superconducting Dipoles," XVth International Conference on High Energy Accelerators, Hamburg. Germany, July 20-24, 1992, Los Alamos National Laboratory document LA-UR-92-2i80.

R. E. Shafer, "Beam Loss Monitoring at SSC and LHC," Los Alamos National Laboratory document LA-UR-9 $:-2302$.

C. R. Rose, C. M. Fortgang, and J. P. Power, "GTA Beamloss-Monitor System," 1992 Linac Conference, Ottawa, Ontario, Canada, August 23-28, 1992.

Los Alamos National Laboratory document LA-UR-92-2630.

F. D. Wells, R. E. Shafer, and j. D. Gilpatrick, "Log-Ratio Beam Position Monitoring at $425 \mathrm{MHz} . " 1992$ Linac Conference, Ottawa, Ontario, Canada, August 23-28, 1992. Los Alamos National Laboratory document LA-UR-92-2666.

J. F. Power, J. D. Gilpatrick, F Neri, and R. B. Shurter, "Characterization of Beam Position Monitors in Two-Dimensions," 1992 Linac Conference, Ottawa, Ontario, Canada. Atgust 23-28, 1992. Los Alamos National Laboratory document LA-UR92-2726.

D. P. Sandoval, R. C. Garcia. J. D. Gilpatrick, M. A. Shinas, R. Wright, V. Yuan, M. E. Zander. and K. F. Johnson, "Video Profile Monitor Diagn stic System for GTA." 1992 Linac Conference, Ottawa. Ontario, Canada, August 23-28, 1992, Los Alamos National Laboratory document LA-UR-92-2727.

J. D. Gilpatrick. H. P. Marquez, J. F. Power, and V. Yuan. "Design and Operation of a Bunched-Beam, Phase-Spread Measurement," 1992 Linac Conference, 
Ottawa, Ontario, Canada, August 23-28, 1992, Los Alamos National Laboratory document LA-UR-92-2728.

R. H. Kraus, Jr., D. B. Barlow, and R. Meyer, "A Variable-Field Permanent-Magnet Dipole for Accelerators," 1992 Linac Conference, Ottawa, Ontario, Canada, August 23-28, 1992, Los Alamos National Laboratory document LA-UR-92-2850.

P. L. Walstrom, "Magnetic Fields from Distributions of Dipoles on Cylindrical Surfaces," Los Alamos National Laboratory document LA-UR-92-3496.

A. Jason, R. Hardekopf, G. Lawrence, R. Macek, R. Pynn, and G. Russell, "LANSCE The Los Alamos "1 MW" Spallation Source," Los Alamos National Laboratory document LA-UR-92-3497.

D. B. Barlow, R. H. Kraus, Jr., P. F. Ruminer, and C. T. Lobb, "Cryogenic and Room Temperature Mapping of GTA Drift-Tube Linac Permanent Magnet Quadrupoles," 1992 NPB Symposium, Argonne, IL, April 1992, Los Alamos National Laboratory document LA-CP-92-210.

P. L. Walstrom, "Three-Dimensional Field Models for High-Order Beam-Optics Codes," 1992 NPB Symposium, Argonne, IL, April 1992, Los Alamos National Laboratory document LA-CP-92-222.

W. P. Lysenko and P. J. Channell, "Testing the New BEDLAM Optics Code," 1992 NPB Symposium, Argonne, IL, April 1992, Los Alamos National Laboratory document LA-CP-92-223.

C. T. Mottershead, "NPB Telescope Design," 1992 NPB Symposium, Argonne, IL, April 1992, Los Alamos National Laboratory document LA-CP-92-252.

\section{AT-4}

E. O. Ballard, K. E. Christensen, and P. P. Prince, "Ground Test Accelerator (GTA) Drift Tube Linac (DTL) Fabrication and Assembly Status," 1992 Linac Conference, Ottawa, Ontario, Canada, August 23-28, 1992, Los Alamos National Laboratory document LA-UR-92-2643.

W. E. Fox, and N. K. Bultman, "Transition Fittings Between Aluminum and Stainless Steel Components of Cryogenic Accelerators," 1992 Linac Conference, Ottawa, Ontario, Canada, August 23-28, 1992, Los Alamos National Laboratory document LA-UR-92-2644.

D. J. Liska, J. Ledford, S. Black, G. Spalek, and J. N. DiMarco, "Design Features of a Seven-Cell High-Gradient Superconducting Cavity," 1992 Linac Conference, Ottawa, Ontario, Canada, August 23-28, 1992, Los Alamos National Laboratory document LA-UR-92-2734.

S. Black and G. Spalek, "Calculation of Mechanical Vibration Frequencies of Stiffened Superconducting Cavities," 1992 Linac Conference, Ottawa, Ontario, Canada, August 23-28, 1992, Los Alamos National Laboratory document LA-UR92-2735.

D. J. Liska, P. Smith, L. Carlisle, T. Larkin, G. Lawrence, and R. Garnett, "Mechanical Features of a 700-MHz Bridge-Coupled Drift Tube Linac," 1992 Linac 
Conference. Ottawa, Ontario, Canada. August 23-28, 1992, Los Alamos National Laboratory document LA-UR-92-2736.

E. O. Bailard, K. E. Christensen, and P. P. Prince, "Ground Test Accelerator (GTA) Drift Tube Linac (DTL) Fabrication and Assembly Status," 1992 NPB Symposium, Argonne, IL, April 1992, Los Alamos National Laboratory document LA-CP-92234.

\section{AT-5}

A. Regan. "Upconverter Module User's Manual," Los Alamos National Laboratory manual LA-12395-M (April 1992).

C. Ziomek, "Vector Modulator Module Manual," Los Alamos National Laboratory manual LA-12396-M (April 1992).

T. Brooks, "Timing Receiver Module User's Manual," Los Alamos National Laboratory manual LA-12401-M (May 1992).

C. Ziomek, "I Controller Module User's Manual," Los Alamos National Laboratory manual LA-12403-M (April 1992).

C. Ziomek, "Q Controller Manual," Los Alamos National Laboratory manual LA12404-M (April 1992).

S. Jachim, "Vector Detector Module User's Manual," Los Alamos National Laboratory manual LA-12406-M (May 1992).

C. Ziomek, "Timing Distribution Module User's Manual," Los Alamos National Laboratory manual LA-12408-M (May 1992).

C. Ziomek. "Low-Level RF LabVIEW Control Software User's Manual," Los Alamos National Laboratory manual LA-12409-M (June 1992).

A. Regan, "Downconverter Module User's Manual," Los Alamos National Laboratory manual LA-124.38-M (June 1992).

M. Curtin. "Envelope Detector Module User's Manual," Los Alamos National Laboratory manual LA-12444-M (May 1992).

M. Curtin, "425-MHz Envelope Detector Module User"s Manual," Los Alamos National Laboratory manual LA-12445-M (May 1992).

C. Ziomek. "Adaptive Feedforward Module User"s Manual." Los Alamos National Laboratory manual LA-12463-M (June 1992).

P. Denney. "Monitor Transmitter Module," Los Alamos National Laboratory manual LA-12473-M (June 1992).

D. Rees, "Test Procedures. SSC 4616 Tetrode Amplifier," Los Alamos National Laboratory document LA-UR-91-3856.

D. Rees, "Conduct of Operations +25-MH/ Tetrode Amplifier," Lios Alamos National Laboratory document L.A-UR-91-3918. 
D. Rees, "University of Twente Klystron Driver High-Power Radio-Frequency System-System Description," Los Alamos National Laboratory document LA-UR92-351.

D. Rees, "University of Twente Klystron Driver High-Power Radio-Frequency System Test Procedures and Results," Los Alamos National Laboratory document LA-UR-92-352.

W. Reass and W. North, "Electrical Design and Operation of a Two-Klystron rf Station for the Los Alamos National Laboratory's Neutral Particle Beam Experiment," 1992 Power Modulator Symposium, Myrtle Beach, SC, June 23-25, 1992, Los Alamos National Laboratory document LA-UR-92-1888.

A. H. Regan, D. Brittain, D. E. Rees, and C. D. Ziomek, "RF System Description for the Ground Test Accelerator Radio-Frequency Quadrupole," 1992 Linac Conference, Ottawa, Ontario, Canada, August 23-28, 1992, Los Alamos National Laboratory document LA-UR-92-2706.

C. D. Ziomek, "Adaptive Feedforward in the LANL rf Control System," 1992 Linac Conference, Ottawa, Ontario, Canada, August 23-28, 1992, Los Alamos National Laboratory document LA-UR-92-2807.

D. Rees and C. Friedrichs, "RF System Work in Support of LAMPF Upgrade," Los Alamos National Laboratory document LA-UR-92-2898.

\section{AT-7}

P. J. Channell, "The Vlasov Equation and POISSON Maps Induced by NonSymplectic Particle Maps," to be published in Physics Letter A, Los Alamos National Laboratory document LA-UR-91-3261.

D. H. Fitzgerald, T. W. Hardek, R. L. Hutson, R. J. Macek, H. A. Thiessen, T. F. Wang, D. V. Neuffer, and E. P. Colton, "Observations of a Fast Transverse Intability in the PSR," to be published in Nuclear Instruments and Methods in Physics Research, Section A, Los Alamos National Laboratory document LA-UR91-3929.

T. Barts and J. Merson, "Users' Notes for POiSSON/SUPERFISH Release 3.0," Los Alamos National Laboratory document LA-UR-91-4140.

B. E. Carlsten, M. V. Fazio, R. J. Faehl, T. J. T. Kwan, D. G. Rickel, and R. M. Stringfield, "Theory and Modeling of a Relativistic Klystron Amplifier with High Space Charge for Microsecond Applications," to be published in SPIE Intense Microwave \& Particle Beams III, Los Alamos National Laboratory document LAUR-91-4150.

M. J. Browman, "Program Power," Los Alamos National Laboratory document LAUR-92-475.

M. J. Browman, "Special MAFIA Postprocessors and Software Tools," Los Alamos National Laboratory document LA-UR-92-476.

B. E. Carlsten, M. V. Fazio, R. J. Faehl, T. J. Kwan, D. G. Rickel, R. D. Ryne, and R. M. Stringfield, "Effect of Intense Space Charge in Relativistic Klystron Amplifiers," Beams 92 Conference, Washington, D. C.. May 25-29, 1992, Los Alamos 
National Laboratory document LA-UR-92-1594.

T-S. F. Wang, "Beam-Loading Stability in Synchrotrons with a Higher rf Harmonic," XVth Int. Conference on High Energy Accelerators, Hamburg. Germany, July 20-24, 1992, Los Alamos National Laboratory document LA-UR-92-2()86.

R. K. Cooper and R. D. Ryne, "Recent Activities in Accelerator Code Development," Advanced Accelerator Concepts Workshop, Port Jefferson, NY, Los Alamos National Laboratory document LA-UR-92-2174.

M. J. Browman and A. Lombardi, "Testing Program Fields," Los Alamos National Laboratory document LA-UR-92-2372.

M. J. Browman, "Notes on Program Fields," Los Alamos National Laboratory document LA-UR-92-2373.

K. C. D. Chan, K. Meier, D. Nguyen, R. Sheffield. T. S. Wang, R. W. Warren, W. Wilson, and L. M. Young, "Design of a Compact Application-Oriented FreeElectron Laser," 1992 Linac Conference, Ottawa, Ontario, Canada, August 23-28, 1992, Los Alamos National Laboratory document LA-UR-92-2515.

B. E. Carlsten, J. C. Goldstein, E. J. Pitcher, and M. J. Schmitt, "Simulations of APEX Accelerator Performance in the New Nonthermalized Photoinjector Regime," 14th Int. Free Electron Laser Conference, Kobe, Japan, August 23-28, 1992. Los Alamos National Laboratory document LA-UR-92-2561.

B. E. Carlsten, J. C. Goldstein, P. G. O’Shea, and E. J. Pitcher, "Measuring Emittance of Nonthermalized Electron Beams from Photoinjectors," 14th Int. Free Electron Laser Conference. Kobe, Japan, August 23-28, 1992, Los Alamos National Laboratory document LA-UR-92-2562.

D. W. Feldman, S. C. Bender, D. A. Byrd, B. E. Carlsten, R. B. Feldman, J. C. Goldstein, R. Martineau, P. G. O'Shea, E. Pitcher, M. J. Schmitt, W. E. Stein, and M. Wilke, "Operation of the APEX Photoinjector Accelerator at 40 MeV," 1992 Linac Conference, Ottawa, Ontario, Canada, August 23-28, 1992, Los Alamos National Laboratory document LA-UR-92-2645.

M. J. Browman, "Special MAFIA Postprocessors for the Analysis of rf Structures," 1992 Linac Conference, Ottawa, Ontario, Canada, August 23-28, 1992, Los Alamos National Laboratory document LA-UR-92-2677.

C. M. Fortgang, "Field Correction in Three-Dimensions for a One-Meter Long Permanent Magnet Wiggler," 1992 Linac Conference, Ottawa, Ontario, Canada, August 23-28. 1992, Los Alamos National Laboratory document LA-UR-92-2678.

M. D. Wilke, P. G. O'Shea, E. J. Pitcher, R. B. Feldman, and A. L.umpkin, "Electron-Beam Diagnostics Development for the Los Alamos FEL Facility," 1992 Linac Conference, Ottawa, Ontario, Canada, August 23-28, 1992, Los Alamos National Laboratory document LA-UR-92-2692.

P. G. O’Shea, B. E. Carlsten, D. W. Feldman, R. B. Feldman, and K. F. McKenna, "Performance of the APEX 40 MeV Photoinjector-Driven Linear Accelerator," Advanced Accelerator Concepts Workshop. Brookhaven National Laboratory, June 14-18. 1992, Los Alamos National Laboratory document LA-UR-92-2725. 
D. Nguyen, D. M. Baca, K. C. D. Chan, R. B. Cheairs, et al., "Initial Performance of Los Alamos Advanced Free Electron Laser," 1992 Linac Conference, Ottawa, Ontario, Canada, August 23-28, 1992, Los Alamos National Laboratory document LA-UR-92-2803.

R. L. Sheffield, B. E. Carlsten, and L. M. Young, "High-Brightness Linac for the Advanced Free-Electron Laser Initiative at Los Alamos," 1992 Linac Conference, Ottawa, Ontario, Canada, August 23-28, 1992, Los Alamos National Laboratory document LA-UR-92-2804.

R. W. Warren and C. M. Fortgang, "Development of a Pulsed Microwiggler System," 1992 FEL Conference, Kobe, Japan, August 24-28, 1992, Los Alamos National Laboratory document LA-UR-92-2805.

P. G. O'Shea and M. Reiser, "Report on the High-Brightness Source Working Group," Advanced Accelerator Concepts Workshop, Brookhaven National Laboratory, June 14-18, 1992, Los Alamos National Laboratory document LA-UR-922809.

R. W. Warren, P. G. O'Shea, S. C. Bender, B. E. Carlsten, J. W. Early, D. W. Feldman, et. al, "Lasing Attempts with a Microwiggler on the Los Alamos FEL," 1992 FEL Conference, Kobe, Japan, August 23-28, 1992, Los Alamos National Laboratory document LA-UR-92-3017.

P. J. Channell and Harry Dreicer, "An Intense Source of Positrons Using a Low Energy Proton Beam." Advanced Accelerator Concepts Workshop, Brookhaven National Laboratory, June 14-18, 1992, Los Alamos National Laboratory document LA-UR-92-3165.

H. Takeda, "Modeling the APLE Injector Solenoid Magnetic Field with the BiotSavart Law," 14th Int. Free Electron Laser Conference, Kobe, Japan, August 25-29, 1992, Los Alamos National Laboratory document LA-UR-92-3231.

G. W. Rodenz, "Users Guide for the Program FRONT," Los Alamos National Laboratory document LA-UR-92-3396.

R. K. Cooper, "Release Notes for POISSON/SUPERFISH 4.0," Los Alamos National Laboratory document LA-UR-92-3397.

\section{AT-8}

A. Kozubal, "Automation from Pictures: Producing Real-Time Code from a State Transition Diagram," ICALEPCS '91 Conference, Tsukuba, Japan, November 11 15, 1991, Los Alamos National Laboratory document LA-UR-91-3340.

L. R. Dalesio, M. R. Kraimer, and A. J. Kozubal, "EPICS Architecture," ICALEPCS '91 Conference, Tsukuba, Japan, November 11-15, 1991, Los Alamos National Laboratory document LA-UR-91-3543.

W. C. Mead, P. S. Bowling, S. K. Brown, R. D. Jones, C. W. Barnes, H. E. Gibson, J. R. Goulding, and Y. C. Lee, "Optimization and Control of a Small-Angle Negative Ion Source Using an On-Line Adaptive Controller Based On the Connectionist normalized Local Spline Neural Network," Los Alamos National Laboratory document LA-UR-91-3670. 
S. K. Brown, P. S. Bowling, H. E. Gibson, R. G. Jacobson, W. B. Ingalls, and D. R. Schmitt, "Progress on the Automation of DTS," Los Alamos National Laboratory document LA-UR-92-0059.

M. E. Zander, R. M. Wright, S. K. Brown, D. P. Sandoval, J. D. Gilpatrick, and H. E. Gibson, "Image Processing and Computer Controls for Video Profile Diagnostic System in the Ground Test Accelerator (GTA)," 1992 Linac Conference, Ottawa, Ontario, Canada, August 23-28, 1992, Los Alamos National Laboratory document LA-UR-92-2354.

R. A. Cole and W. H. Atkins, "Real-Time Data Archiving for GTA," 1992 Linac Conference, Ottawa, Ontario, Canada, August 23-28, 1992, Los Alamos National Laboratory document LA-UR-92-2420.

M. W. Stettler, M. E. Thuot, L. R. Dalesio, R. A. Cole, C. B. Fite, G. E. Slentz, and D. S. Warren, "A Distributed Timing System for Synchronizing Control and Data Correlation,” 1992 Linac Conference, Ottawa, Ontario, Canada, August 23-28, 1992, Los Alamos National Laboratory document L.A-UR-92-2422.

W. H. Atkins and R. G. Jones, "The Run Permit Protection System for GTA," 1992 Linac Conference, Ottawa, Ontario, Canada, August 23-28, 1992, Los Alamos National Laboratory document LA-UR-92-2737.

M. E. Zander, R. M. Wright, S. K. Brown, D. P. Sandoval, J. D. Gilpatrick, and H. E. Gibson, "Image Processing and Computer Controls for Video Profile Diagnostic System in the Ground Test Accelerator (GTA)," 1992 Neutral Particle Beam Technical Symposium, Argonne, IL, April 27-30, 1992, Los Alamos National Laboratory document LA-CP-92-266.

S. K. Brown, W. C. Mead, P. S. Bowling, and R. D. Jones, "Real-Time Optimization and Control of a Small Angle Ion Source Using an Adaptive Neural Network Controller," Neutral Particle Beam Technical Symposium, Argonne, IL, April 2730, 1992, Los Alamos National Laboratory document LA-CP-92-304.

\section{AT-9}

M. V. Fazio, "Long Pulse Relativistic Klystron High Power Microwave Source Research-1991 Progress Report," Los Alamos National Laboratory document LAUR-92-744.

R. F. Hoeberling, "Electromagnetic Numerical Code Assessment-Final Report," Los Alamos National Laboratory document LA-UR-92-1179.

J. Kinross-Wright, "High Power Microwave Testing and Source Report," Los Alamos National Laboratory document LA-UR-92-1180.

F. VanHaaften, A. Erickson, and K. Rust, "A Chronology of the BANSHEE Thyratron Switch Medulator Development," Los Alamos National Laboratory document LA-UR-92-1447.

M. V. Fazio, B. E. Carlsten, R. J. Faehl, W. B. Haynes, R. F. Hoeberling, T. J. T. Kwan, D. G. Rickel, F. W. VanHaaften, R. M. Stringfield, et al., "The Experimental and Theoretical Development of a One Gigawatt, Repetitively Pulsed, One Microsecond Pulse Length, High Current Relativistic Klystron and Modulator," Beams 92-Ninth International Conf. on High Power Particle BEAMS, Washington, DC, 
May 25-29, 1992, Los Alamos National Laboratory document LA-UR-92-1612.

R. M. Stringfield, R. M. Wheat, D. J. Brown, M. V. Fazio, J. Kinross-Wright, B. E. Carlsten et al., "Large Orbit Gyroklystron Development at Los Alamos," Beams 92Ninth International Conf. on High Power Particle BEAMS, Washington, DC, May 25-29, 1992, Los Alamos National Laboratory document LA-UR-92-1656.

R. M. Stringfield, R. J. Faehl, M. V. Fazio, R. F. Hoeberling et al., "The Development of a One Microsecond Pulse Length, Repetitively Pulsed, High Power Modulator and a Long-Pulse Electron Beam Diode for the Production of Intense Microwaves," Beams 92-Ninth International Conf. on High Power Particle BEAMS, Washington, DC, May 25-29, 1992, Los Alamos National Laboratory document LA-UR-92-1695.

F. Van Haaften, "BANSHEE-High Voltage Repetitively Pulsed Electron Beam Driver," 1992 Power Modulator Symposium, Myrtle Beach, SC, June 23-25, 1992, Los Alamos National Laboratory document LA-UR-92-1716.

S-J. Han, "Stability of an Imploding Spherical Shell," to be published in Physical Review A, Los Alamos National Laboratory document LA-UR-92-1965.

M. V. Fazio, B. L. Freeman, R. F. Hoeberling, J. Kinross-Wright, D. G. Rickel, and R. M. Stringfield, "A Microsecond Pulse-Length, Frequency-Stabilized Virtual Cathode Oscillator Using a Resonant Cavity," to be published in IEEE Transactions on Plasma Science, Special Issue on High Power Microwave Generation, Los Alamos National Laboratory document LA-UR-92-2823.

\section{AT-10}

V. W. Yuan, C. D. Bowman, J. D. Bowman, J. E. Bush, P. P. J. Delheif, C. M. Frankle, C. R. Gould, et al., "Parity Nonconservation in Polarized-Neutron Transmission through ${ }^{139}$ La," Physical Review C, 44, Number 5, 2187-2194, (1991).

C. M. Frankle, J. D. Bowman, J. E. Bush, V. W. Yuan, et al., "Parity Nonconservation for Neutron Resonances in ${ }^{232}$ Th," Physical Review C, 46, Number 2, 778-787, (August 1992).

X. Zhu, J. D. Bowman, C. D. Bowman, J. E. Bush, V. W. Yuan, et al., "Parity Nonconservation for Neutron Resonances in ${ }^{238} \mathrm{U}$," Physical Review C, 46, Number 2, 768-777, (August 1992).

C. M. Frankle, J. D. Bowman, J. E. Bush, V. W. Yuan, et al., "Parity Nonconservation for the 0.88-eV Neutron Resonance in ${ }^{81} \mathrm{Br}$," Physical Review $C$, 46, Number 4, 1542-1545, (October 1992).

D. G. Haase, J. D. Bowman, P. P. J. Delheij, V. W. Yuan, et al., "Depolarization of Neutrons in Ferromagnetic Holmium by Means of Enhanced Nuclear Parity Violation in ${ }^{139}$ LA," Physical Review B, 46, Number 18,11 290-11 294 (November 1992).

H. V. Smith, Jr., J. D. Sherman, C. Geisik, and P. Allison, "H- Temperature Dependencies in a Penning Surface-Plasma Source," 4 th Int. Conference on Ion Sources, Bensheim, Germany, September 30-October 4, 1991, Los Alamos National Laboratory document LA-UR-91-2802. 
H. V. Smith, Jr., P. Allison, C. Geisik, S. D. Orbesen, et al., "Status of $8 \mathrm{X} \mathrm{H}^{-}$Ion Source Development," Los Alamos National Laboratory document LA-UR-921950.

H. V. Smith and Paul Allison, "Suppression of the e- Coextracted from a Penning Surface-Plasma H-Source," 1992 Linac Conference, Ottawa, Ontario, Canada, August 23-28, 1992, Los Alamos National Laboratory document LA-UR-92-2553.

K. F. Johnson, O. R. Sander, W. H. Atkins, G. O. Bolme, et al.. "Commissioning of the Ground Test Accelerator RFQ," 1992 Linac Conference, Ottawa, Ontario, Canada, August 23-28, 1992, Los Alamos National Laboratory document LA-UR92-2703.

K. Johnson, O. Sander, W. Atkins, G. Bolme, S. Brown, R. Connoly, R. Garnett, J. Gilpatrick, F. Guy, W. Ingalls, C. Little, R. Lohsen, S. Lloyd, G. Neuschaefer, J. Power, K. Saadatmand, D. Sandoval, R. Stevens, G. Vaughn, E. Wadlinger, R. Weiss, and V. Yuan, "Commissioning of the Ground Test Accelerator Intertank Matching Section," 1992 Linac Conference, Ottawa, Ontario, Canada, August 2328, 1992, Los Alamos National Laboratory document LA-UR-92-2704.

R. Connolly, K. F. Johnson, and V. Yuan, "A Correction for Emittance-Measurement Errors Caused by Finite Slit and Collector Widths," 1992 Linac Conference, Ottawa, Ontario, Canada, August 23-28, 1992, Los Alamos National Laboratory document LA-UR-92-2715.

O. R. Sander, W. H. Atkins, G. O. Bolme, S. Bowling, S. Brown, R. Cole, et al., "Commissioning the GTA Accelerator," 1992 Linac Conference, Ottawa, Ontario, Canada, August 23-28, 1992, Los Alamos National Laboratory document LA-UR92-2716.

D. P. Sandoval, R. C. Garcia, J. D. Gilpatrick, M. A. Shinas, R. Wright, V. Yuan, M. E. Zander, and K. F. Johnson, "Video Profile Monitor Diagnostic System for GTA," 1992 Linac Conference. Ottawa, Ontario, Canada, August 23-28, 1992, Los Alamos National Laboratory document LA-UR-92-2727.

G. O. Bolme, P. M. Denney, W. D. Gutscher, S. P. Jachim, et al., "Analysis of High-Power Conditioning for Accelerator Cavities Using a Six-Port Reflectometer," 1992 Linac Conference, Ottawa, Ontario, Canada, August 23-28, 1992, Los Alamos National Laboratory document LA-UR-92-2739.

C. M. Frankle, J. D. Bowman, S. I. Penttila, S. J. Seestrom, S. H. Yoo, V. W. Yuan et al, "Measurement of Parity Violation in Compound Nuclear Resonances Using Epithermal Polarized Neutrons." to be published in NIM, Los Alamos National Laboratory document LA-UR-92-2740.

J. D. Bowrnan and V.W. Yuan, "Measurement of Parity Violation in Compound Nuclear Resonances using Epithermal Polarized Neutrons," to be published in Nuclear Instruments and Methods, Los Alamos National Laboratory document LAUR-92-2833.

V.W. Yuan. R.C. Connolly, R.C. Garcia, K.F. Johnson, K. Saadatmand, O.R. Sander, D.P. Sandoval, and M.A. Shinas, "Measurement of Longitudinal Phase Space in an Accelerated $\mathrm{H}^{-}$Beam Using a Laser-Induced Neutralization Method," to be published in Nuclear Instruments and Methods, Los Alamos National Laboratory document LA-UR-92-2892. 
H. V. Smith, Jr., and P. Allison, "Electron Suppression in the $\mathrm{H}^{-}$Beam from a Penning Surface-Plasma Source," to be published in Review of Scientific Instruments, Los Alamos National Laboratory document LA-UR-92-3337.

J. D. Sherman, H. V. Smith. Jr., C. Geisik, and P. Allison, "H- Temperature Dependencies in a Penning Surface-Plasma Source," Neutral Particle Beam Technical Symposium, Argonne, IL, April 27-30, 1992, Los Alamos National Laboratory document LA-CP-92-217.

E. A. Meyer, C. Bridgman, R. J. Grieggs, D. R. Schmittt, and J. D. Schneider, "Improved Pulsed Gas Injection System for the GTA 4X Ion Source," Neutral Particle Beam Technical Symposium, Argonne, IL, April 27 - May 1, 1992, Los Alamos National Laboratory document LA-CP-92-227.

L. B. Dauelsberg and C. Vigil, "Alignment Procedures for the GTA DTL Modules," Neutral Particle Beam Technical Symposium, Argonne, IL, April 27-30, 1992, Los Alamos National Laboratory document LA-CP-92-228.

C. Geisik, D. R. Schmitt, J. D. Schneider, and J. E. Stelzer, "Computer System for the High Current Test Stand," Neutral Particle Beam Technical Symposium, Argonne, IL, April 27-30, 1992, Los Alamos National Laboratory document LACP-92-232. 


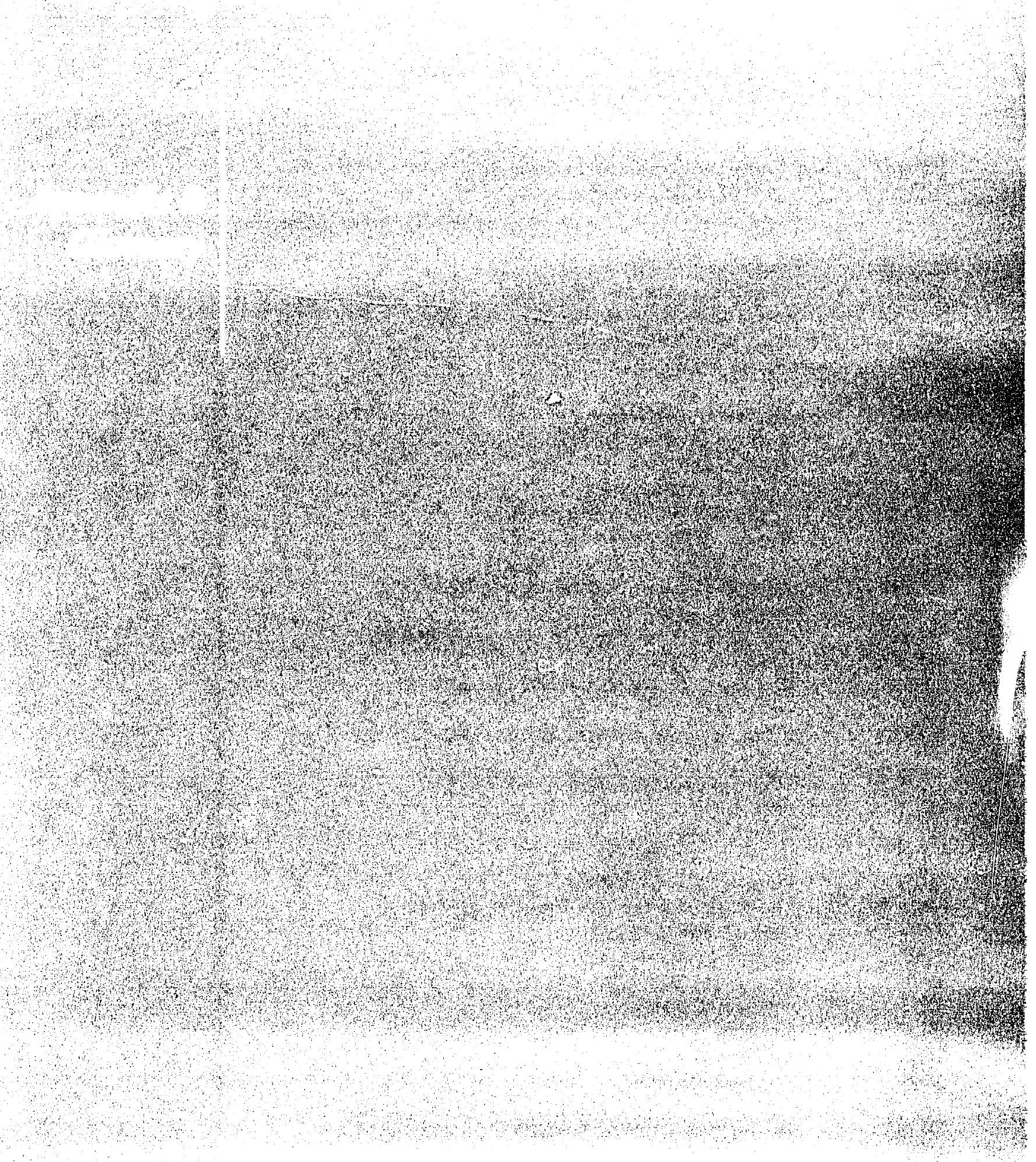




\begin{tabular}{|c|c|}
\hline $\mathrm{ABC}$ & Accelerator Based Conversion \\
\hline AFEL & advanced free-electron laser \\
\hline APEX & APLE Prototype EXperiment \\
\hline APLE & Average Power Laser Experiment \\
\hline APS & Advanced Photon Source \\
\hline APT & Accelerator Production of Tritium \\
\hline ARES & Advanced Rescarch Electromagnetic Simulator \\
\hline $\mathrm{A} \mathrm{T}-4$ & Acceleration Design and Engineering \\
\hline AT -5 & RF Technology \\
\hline AT-7 & Accelerator Theory \& FEL Technology \\
\hline AT-8 & Accelerator Controls and Automation \\
\hline ATW & Accelerator Transmutation of Waste \\
\hline $\mathrm{AXY}$ & $A=$ high-power accelerator, $X Y=$ represent the specific application \\
\hline BCDTL & bridge-coupled drift tube linac \\
\hline BEAR & Beam Experiment Aboard a Rocket \\
\hline BESAC & Basic Energy Sciences Advisory Committec \\
\hline BNL & Brookhaven National Laboratory \\
\hline BOP & balance of plant \\
\hline $\mathrm{CAD}$ & computer aided design \\
\hline CAE & computer aided engineering \\
\hline $\mathrm{CCL}$ & coupled-cavity linac \\
\hline CCS & cryogenic cooling system \\
\hline CEBAF & Continuous Beam Accelerator Facility \\
\hline CERN & European Center for Nuclear Research \\
\hline CLS-4 & Photo Chemistry and Photo Physics \\
\hline CLS-6 & Advanced Laser and Systems Technology \\
\hline CPU & central processing unit \\
\hline CRADA & Cooperative Research and Development Agreement \\
\hline CRL & Chalk River Laboratory \\
\hline CTEN & Combined Thermal/Epithermal Neutron \\
\hline$c w$ & continuous wave \\
\hline CWDD & continuous wave deuterium demonstrator \\
\hline DARHT & Dual-axis Radiographic Hydrodynamic Test \\
\hline $\mathrm{dB}$ & decibel \\
\hline $\mathrm{dc}$ & direct current \\
\hline DCM & downconverter module \\
\hline $\mathrm{DCN}$ & Document Change Notice \\
\hline DEWPOINT & Directed Energy Weapons Power Integration \\
\hline DNA & Defense Nuclear Agency \\
\hline $\mathrm{DoD}$ & Department of Defense \\
\hline $\mathrm{DOE}$ & Department of Energy \\
\hline DP & Defense Programs \\
\hline dt & drift tube \\
\hline DTL & drift tube linac \\
\hline DTS & discharge lest stand \\
\hline $\mathrm{ECAD}$ & electronic computer aided design \\
\hline EEV & English Electric Valve \\
\hline $\mathrm{EM}$ & electromagnetic \\
\hline EMP & electromagnetic pulse \\
\hline EMQ & electromagnet quadrupoles \\
\hline EMR & electromagnetic radiation \\
\hline EPICS & Experimental Physics and Industrial Control System \\
\hline
\end{tabular}




\begin{tabular}{|c|c|}
\hline ERAB & Energy Research Advisory Board \\
\hline ES\&H & Enviroment, safety, and health \\
\hline ESNIT & Energy Selective Neutron Irradiation Test \\
\hline FEL & free-electron laser \\
\hline FMIF & Fusion Materials Irradiation Facility \\
\hline FOX & far-field optics experiment \\
\hline FWBPM & flying-wire beam-profile measurement \\
\hline FWHM & full-width-at-half-maximum \\
\hline $\mathrm{G}$ & gauss \\
\hline GAC & Grumman Aerospace Corporation \\
\hline GFI & ground fault interrupter \\
\hline GL & gradient length \\
\hline GPR & ground penetrating radar \\
\hline GTA & Ground Test Accelerator \\
\hline HAM & hybrid amplifier module \\
\hline HCTS & high-current test stand \\
\hline HEBT & high-energy beam transport \\
\hline HIBAF & high-brightness accelerator FEL \\
\hline HIPERGPR & high-performance ground penetrating radar \\
\hline HPCTB & High-Power Cryogenic Test Bed \\
\hline HPM & high-power microwave \\
\hline HPP & high-pulse power \\
\hline HPRF & high-power radio-frequency system \\
\hline $1 \& C$ & instrumentation and control \\
\hline ICN & Integrated Computing Network \\
\hline IMS & intermediate matching section \\
\hline INEL & Idaho National Engineering Laboratory \\
\hline IOC & input/output controller \\
\hline ITF & Integrated Test Facility \\
\hline JAERI & Japan Atomic Energy Research Institute \\
\hline LAACG & Los Alamos Accelerator Code Group \\
\hline LAMPF & Clinton P. Anderson Meson Physics Facility \\
\hline LANSCE II & Manual Lujan, Jr. Neutron Scattering Center II \\
\hline LANSCE & Manual Lujan, Jr. Neutron Scattering Center \\
\hline LBL & Lawrence Berkeley Laboratory \\
\hline LDRD & laboratory-directed research and development \\
\hline LEB & low-energy booster \\
\hline LEBT & low-energy beam transport \\
\hline LEP & large electron positron collider \\
\hline LINDA & laser induced neutralization diagnostics approach \\
\hline LLNL & Lawrence Livermore National Laboratory \\
\hline LLRF & low-level rf \\
\hline LOG & large orbit gyrotron \\
\hline LPCTB & Low-Power Cryogenic Test Bed \\
\hline LVDT & linear variable differential transducer \\
\hline MEBT & moderate energy beam transport \\
\hline MMTW & multiple-magnet taut-wire \\
\hline MP & Medium Energy Physics Division \\
\hline MSDS & Material Safety Data Sheet \\
\hline
\end{tabular}


MST-7 Materials Science and Technology: Polymers and Coatings

NCLR

National Center for Laser Research

NCMS

NERSC

National Center for Manufacturing Sciences

NLC

National Energy Research Supercomputer Center

NOP

NP

Next Linear Collider

normal operating procedures

NPB

NPBSE

New Production

neutral particle beam

NSI

neutral particle beam space experiment

National Security Information

OFE Office of Fusion Energy

ORNL Oak Ridge National Laboratory

OTR optical-transition radiation

P-15

Neutron Measurements Group

PAC

Particle Accelerator Conference

PEIS

PET

PIC

Programmatic Environmental Impact Statement

positron-emission tomography

particle-in-cell

PID

PMQ

POP

PSII

PSR

proportional, integral, and differential

permanent-magnet quadrupole

proof-of-principle

plasma source ion implantation

proton storage ring

QA

quality assurance

R\&D research and development

RDBMS relational database management system

rf

radio frequency

RFQ radio-frequency quadrupole

RKA relativistic kylstron amplifier

rms root-mean-square

RPSS Radiation Protection Safety System

RRR residual resistance ratio at $4 \mathrm{~K}$

RSM3 rotating sample magnetic moment mapper

SAIC Science Application International Corporation

SAIS

SC

small angle ion source

superconducting

SCR

SDIO

SLAC

SNL

SNSI

silicon controlled rectifier

Strategic Defense Initiative Organization

Stanford Linear Accelerator Center

Sandia National Laboratories

Secret National Security Information

SOP

standard operating procedures

SRD Secret Restricted Data

SSC

Superconducting Super Collider

SSCL

SST

Superconducting Super Collider Laboratory

STATS

Separations Technology and Transmutation Systems

$\mathrm{T}$

temperature

TTI

Technology Transfer Initiative 


$\begin{array}{ll}\text { UPM } & \text { upconverter module } \\ \text { USASDC } & \text { United States Army Strategic Defense Command } \\ \text { VDM } & \text { vector detector module } \\ \text { VFPMQ } & \text { variable field permanent magnet quadrupole } \\ \text { VLE } & \text { vulnerability, lethality, and effects (VL\&E) } \\ \text { VME } & \text { versa-module European } \\ \text { VXI } & \text { VME External Interface } \\ \text { VSWR } & \text { voltage standing wave ratio } \\ \text { WEC } & \text { Westinghouse Electric Corporation } \\ \text { WEMPE 4 } & \text { wideband electromagnetic pulse environment } \\ & \\ \text { X-1 } & \text { Inertial Fusion and Plasma Theory } \\ \text { XUV } & \text { extreme ultraviolet }\end{array}$



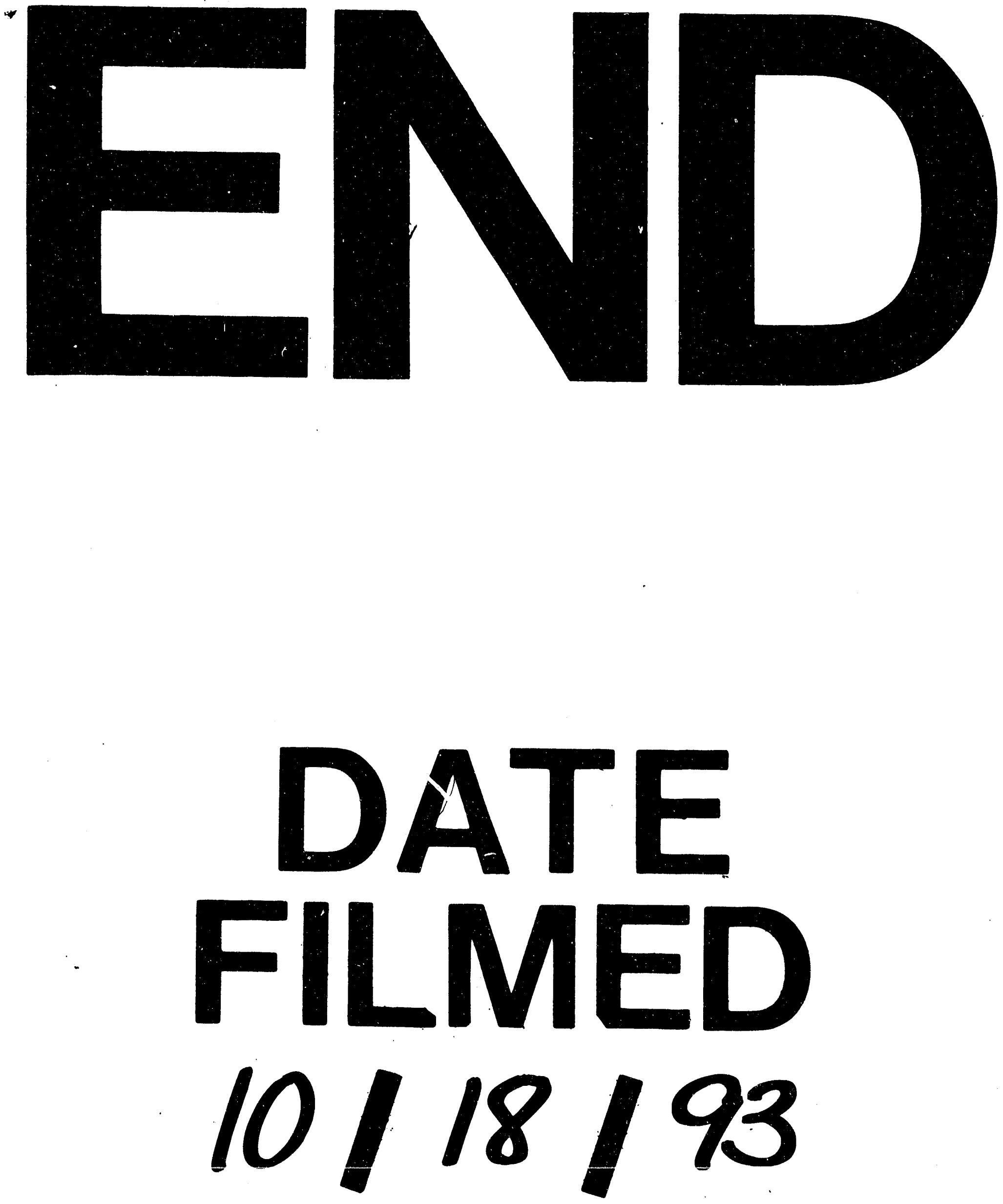
